Christian Schiller

\title{
Staatsausgaben und crowding-out-Effekte
}




\section{Christian Schiller}

\section{Staatsausgaben und crowding-out-Effekte}

Unter dem Eindruck der Erfahrungen des letzten Jahrzehnts und vorher schon aufgrund theoretischer Überlegungen sind mehr und mehr Zweifel aufgekommen, ob eine finanzwirtschaftliche Nachfragesteuerung ein taugliches Mittel gegen Unterbeschäftigung ist. Ziel der vorliegenden Arbeit ist es, die theoretischen Erwägungen aufzuarbeiten und im Hinblick auf ihre Voraussetzungen und deren Plausibilität zu überprüfen.

Christian Schiller wurde 1952 in Dresden geboren. Er studierte von 1972 bis 1978 Volkswirtschaftslehre an der Johannes Gutenberg-Universität Mainz. Nach der Diplom-Prüfung im Frühjahr 1978 promovierte er bei Professor Dr. Kurt Schmidt am Institut für Finanzwissenschaft der Universität Mainz. Der Autor ist seit 1978 am selben Institut als wissenschaftlicher Mitarbeiter tätig. 
Staatsausgaben und crowding-out-Effekte 


\section{FINANZWISSENSCHAFTLICHE SCHRIFTEN}

Herausgegeben von den Professoren

Albers, Krause-Junk, Littmann, Oberhauser, Pohmer, Schmidt

Band 21

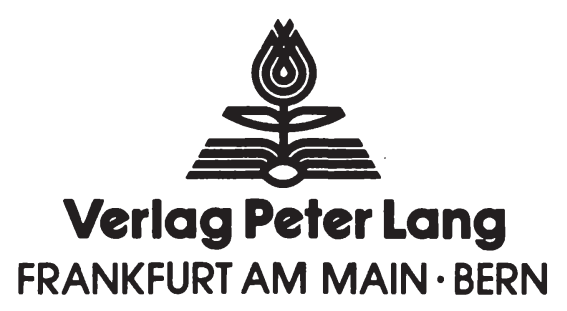




\section{Christian Schiller}

\section{Staatsausgaben und crowding-out-Effekte}

Zur Effizienz einer

Finanzpolitik keynesianischer Provenienz

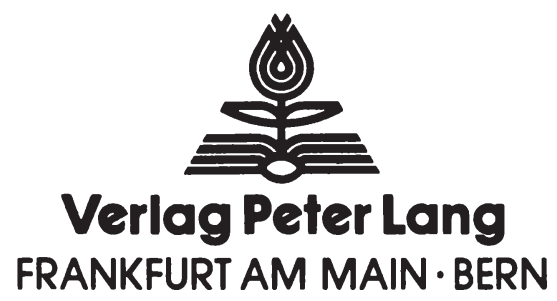


CIP-Kurztitelaufnahme der Deutschen Bibliothek

\section{Schiller, Christian:}

Staatsausgaben und crowding-out-Effekte : zur

Effizienz e. Finanzpolitik keynesian. Provenienz /

Christian Schiller. - Frankfurt am Main ; Bern :

Lang, 1983.

(Finanzwissenschaftliche Schriften ; Bd. 21)

ISBN 3-8204-7503-6

NE: GT

Open Access: The online version of this publication is published on www.peterlang.com and www.econstor.eu under the international Creative Commons License CC-BY 4.0. Learn more on how you can use and share this work: http://creativecommons.org/licenses/by/4.0.

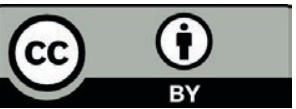

This book is available Open Access thanks to the kind support of ZBW - Leibniz-Informationszentrum Wirtschaft.

ISSN 0170-8252

ISBN 3-8204-7503-6

ISBN 978-3-631-75197-8 (eBook)

(C) Verlag Peter Lang GmbH, Frankfurt am Main 1983

Alle Rechte vorbehalten.

Nachdruck oder Vervielfältigung, auch auszugsweise, in allen Formen wie Mikrofilm, Xerographie, Mikrofiche, Mikrocard, Offset verboten.

Druck und Bindung: fotokop wilhelm weihert $K G$, darmstadt 


\section{Vorwort}

Die vorliegende Abhandlung entstand während meiner Tätigkeit als wissenschaftlicher Mitarbeiter bei Herrn Professor Dr. Kurt Schmidt am Institut für Finanzwissenschaft der Universität Mainz. Sie wurde im Sommersemester 1982 vom Fachbereich Rechts- und Wirtschaftswissenschaften der Johannes GutenbergUniversität Mainz als Dissertation angenommen.

Meinem verehrten Lehrer, Herrn Professor Dr. Kurt Schmidt, möchte ich an dieser stelle für die vielen Anregungen und Verbesserungsvorschläge aufrichtigen Dank aussprechen. Er hat auch die Veröffentlichung in der Reihe "Finanzwissenschaftliche Schriften" ermöglicht. Herrn Professor Dr. Bartmann bin ich dankbar dafür, daB er (trotz zeitlichen Engpasses) das Koreferat übernommen hat. Schlieblich möchte ich meiner Frau und Fachkollegin für die kritische Durchsicht des Manuskriptes danken.

Mainz, im Herbst 1982 Christian Schiller 
Christian Schiller - 978-3-631-75197-8

Downloaded from PubFactory at 01/11/2019 07:15:17AM

via free access 
INHALTSVERZEICHNIS

Seite

Vorwort

Inhaltsverzeichnis

VII

Erster Teil: Grundlagen der crowding-outAnalyse

1. Kapitel: Problemstellung und Aufbau der Arbeit

A. Problemstellung

B. Aufbau der Arbeit

2. Kapitel: Dogmenhistorische Wurzeln der crowding-out-These

A. Die crowding-out-These als Bestandteil der klassischen Theorie

B. Negierung der crowding-out-These in der keynesianischen Theorie

C. Renaissance der crowding-out-These in der monetaristischen Theorie

3. Kapitel: Formen des crowding-out

A. Hauptelemente des Ablaufschemas finanzwirtschaft]icher MaBnahmen

B. Impuls: Direktes und indirektes crowdingout

C. Transmission

1. Ex ante und ex post crowding-out

2. Kurzfristiges, mittelfristiges und langfristiges crowding-out

3. Finanzielles und güterwirtschaftliches crowding-out 
D. Effekt

1. Partielles, vollständiges und übermäBiges crowding-out

24

2. Reales und nominelles crowding-out

4. Kapitel: Zum Untersuchungsrahmen

A. Impuls

1. Die Finanzierungsrestriktion des Staates

2. Wirkungskonzept: Budgetinzidenz

3. Ausgabenart und Finanzierungsart: Transformationsausgaben und Kapitalmarktfinanzierung

4. Zur Abgrenzung finanzwirtschaft.ticher Impulse von geldpolitischen Impulsen

5. Zusammenfassung

B. Transmission

1. Betrachtungszeitraum: kurze und mittlere Frist

2. Gesamtwirtschaftliche Ausgangslage

a) Unterbeschäftigung

b) Nachfrageseitige und angebotsseitige Ursachen von Unterbeschäftigung

C. Effekt

1. Verdrängungsindikator: Privatausgaben

2. Zur "Null-Linie" von crowding-out

3. Crowding-out-Effekt als Gesamtund als Teileffekt

D. Finanzwirtschaftliches demand management im Grundmodel]

1. Grundmodell

2. Wirkungsanalyse

a) Gesamtausgabeneffekt

b) Privatausgabeneffekte

3. Zur keynesianischen position 
2. Kapitel: Vermögenseffekte 61

A. Das konventionelle Portfolio-crowding-out 61

1. Darstellung 61

a) Entstehungsphase des Defizits 61

b) Reine Finanzierungsphase des Defizits 62

2. Berücksichtigung im Grundmodell 63

a) Entstehungsphase des Defizits 63

b) Reine Finanzierungsphase des Defizits 66

3. Würdigung

a) Zur Vermögensabhängigkeit der Geldnachfrage

b) Berücksichtigung des Lerner-Effekts 70

c) Kontraktive Vermögenseffekte und langfristige Stabilität

d) $\mathrm{zu}$ den Substitutionsbeziehungen zwischen den Vermögenskomponenten

aa) Grundlage: ein erweitertes makroökonomisches Portfoliomodell

bb) Makroökonomisches Portfolio und private Ausgaben

cc) Portfolio-crowding-out oder Portfollio-crowding-in?

e) Tax discounting

f) Staatsverschuldung und privates Vermögen: definitorischer Ansatz versus Sparansatz

4. Exkurs: Quellen- versus Fontänentheorie

a) Darstellung 98

b) Bedingungen der Fontänentheorie $\quad 100$

B. Sonstige Vermögenseffekte 102

1. Ursachen und Transmissionskanäle von Vermögenseffekten

2. Vermögensmengeneffekte 104

3. Vermögenswerteffekte 109 
3. Kapitel: Ausgabeneffekte 113

A. Gegenstand der Uberlegungen 113

B. Zum direkten Staatsausgabeneffekt 114

C. Zum einkommensinduzierten Konsumeffekt 116

1. Permanentes Einkommen als Bestimmungsgrund der privaten Konsumausgaben 116

2. రffentliche Güter als Bestimmungsgrund der privaten Ausgaben

a) Die Staatsausgaben im Güterkreislauf

b) Befriedigung bisher privat gedeckter Güternachfrage durch offentliche Leistungen

c) Die Bailey-These 122

aa) Die Baileysche Konsumfunktion 122

bb) Ausgabenmultiplikatoren 123

cc) Einschränkungen 125

d) Die David/Scadding-These 127

aa) Annahmen 127

bb) Stabilisierungspolitische Konsequenz: vollständiges, direktes crowding-out

cc) Kritische Würdigung 130

D. Zum Akzelerationseffekt 133

1. Investitionsattentismus: angebotsseitige und nachfrageseitige Ursachen 133

2. Staatlicher Handlungsbedarf bei angebotsseitig und nachfrageseitig bestimmter Investitionszurückhaltung

4. Kapitel: Preiseffekte

A. Reales crowding-out

B. Zur Abhängigkeit der realen Güternachfrage der Privaten vom Preisniveau

C. Preisniveau und kreditär finanzierte staatliche Nachfrageimpulse

1. Finanzwirtschaftlich verursachter Nachfragesog

a) Monetärer Nachfragesog 142

b) Güterwirtschaftlicher Nachfragesog 148

2. Finanzwirtschaftlich verursachter Angebotsdruck 
5. Kapitel: Erwartungseffekte 155

A. Erwartungen als Bestimmungsgrund der privaten Ausgaben

B. Zum Bilden von Erwartungen 156

C. Crowding-out durch Erwartungseffekte 158

1. Finanzwirtschaftliches demand management und Zukunftsvertrauen 158

a) Die These von Keynes 158

b) Die These des Sachverständigenrates 162

2. Finanzwirtschaftliches demand management und rationale Preiserwartungen

a) Konsequenzen rationaler Erwartungen

b) Die These der Rationalisten

aa) Die Friedman/Phelps-These und ihre Erweiterung durch die Rationalisten

bb) Darstellung

cc) Kritik 
Christian Schiller - 978-3-631-75197-8

Downloaded from PubFactory at 01/11/2019 07:15:17AM

via free access 
Mylord, die Mühe konntet Ihr Euch sparen.

Schiller, Maria Stuart

Erster Teil: Grundlagen der crowding-out-Analyse

Erstes Kapitel: Problemstellung und Aufbau der Arbeit

A. Problemstellung

Als am 8. Juni 1967 in der Bundesrepublik Deutschland das

"Gesetz zur Förderung der Stabilität und des Wachstums der wirtschaft" verabschiedet worden ist, hatte die "keynesianische Botschaft" von der Machbarkeit der Konjunktur mittels finanzwirtschaftlichem demand management auch hierzulande ihren gesetzlichen Niederschlag gefunden. Heute wird unter Wirtschaftstheoretikern zwar heftig darüber debattiert, ob diese Botschaft von Keynes selbst oder von seinen Interpreten, hauptsächlich Hicks, Hansen und Klein, verkündet wurde (und ob Keynes überhaupt ein Keynesianer war) 1), gleichwohl spielte keynesianisches Gedankengut bei der Politikberatung in allen industrialisierten Ländern der westlichen Welt die dominierende Rolle ${ }^{2}$. Die keynesianische Botschaft läBt

1) Diese Diskussion geht zurück auf Leijonhufvud, A., On Keynesian Economics and the Economics of Keynes, New York, 1968; einen Uberblick vermittelt Grossman, H.J., Was Keynes a "Keynesian"? A Review Article, in: Journal of Economic Literature, 1972, S. 26 ff.

2) In der Bundesrepublik Deutschland ist die keynesianische Botschaft vergleichsweise spät von den wirtschaftspolitikern aufgegriffen worden, nämlich erst mit der Rezession 1966/67. Zur Umsetzung der keynesianischen Rezeptur in die wirtschaftspolitische Praxis hierzulande und in den Vereinigten Staaten von Amerika sowie in GroBbritannien vgl. Spahn, H.-P., Keynes in der heutigen Wirtschaftspolitik, in: Bombach, G., Ramser, H.-J., Timmermann, M., Wittmann, W. (Hrsg.), Der Keynesianismus I, Theorie und Praxis keynesianischer Wirtschaftspolitik, Berlin, Heidelberg, New York, 1976, S. 219 ff. 
sich in groben Strichen wie folgt zusammenfassen ${ }^{11}$ :

- Unterbeschäftigung ist hauptsächlich Folge einer unzureichenden gesamtwirtschaftlichen Guternachfrage $\left(Y^{d}\right)$. Diese setzt sich aus den Ausgaben der privaten Konsumenten (C) und Investoren (I) sowie aus der staatlichen Güternachfrage (G) zusammen ${ }^{2)}$ :

$$
(1-1-1) \quad Y^{d}=C+I+G
$$

- Der private Sektor ist instabil; die Privaten tätigen nicht "automatisch" stets so viele Ausgaben, daB alle Arbeitswilligen beschäftigt werden können.

- In diesem Fall muB und kann der staat die Gesamtausgaben so beeinflussen, daB diese ein vollbeschäftigungskonformes Niveau erreichen. Dazu bieten sich die Transformationsausgaben an. Es ist jedoch nicht nötig, daB der staat die ganze Güternachfragelücke selbst mit zusätzlichen Transformationsausgaben auffült; denn mit der Entfaltung eigener Güternachfrage regt er via Multiplikator- und Akzeleratoreffekte die Privaten dazu an, ihre Ausgabenpläne ebenfalls aufzustocken.

Zur gleichen Zeit, als in der Bundesrepublik Deutschland mit der Verabschiedung des "Gesetz zur Förderung der Stabilität und des Wachstums in der Wirtschaft" hohen Erwartungen in die Wirksamkeit einer keynesianisch gestalteten Finanzpolitik Ausdruck gegeben wurde, hat sich in den Vereinigten

1) Vgl. z.B. Landmann, P., Keynes in der heutigen Wirtschaftstheorie, in: Bombach, G., Ramser, H.-J., Timmermann, M., Wittmann, W. (Hrsg.), Der Keynesianismus I, Theorie und Praxis keynesianischer Wirtschaftspolitik, Berlin, Heidelberg, New York, 1976, S. $137 \mathrm{ff}$. sowie für einen knappen Uberblick Littmann, K., Stand der Stabilisierungspolitik, in: Quartalshefte der osterreichischen Girozentrale, $2-3 / 1978$, s. 36 ff.

2) Die Analyse ist - wle ublich - etwas vergrobert; die gesamtwirtschaftliche Güternachfrage umfaBt zusătzlich noch die Nachfrage nach $\mathrm{Zwischenprodukten,} \mathrm{ist} \mathrm{also} \mathrm{die} \mathrm{Summe}$ aus der Zwischenprodukt- und Endproduktnachfrage. 
Staaten von Amerika eine Gruppe von Nationalobonomen - die Monetaristen - formiert, welche die Leistungsfahigkelt elner Stabilisierungspolitik keynesianischer Provenienz in Frage stellt. In erster Linie Milton Friedman ${ }^{1)}$ und Okonomen der St. Louls Bank ${ }^{2}$ propagierten, daB eine keynesianisch angelegte Finanzpolitik im Hinblick auf das stabilislerungszlel unwirksam sel; denn ein finanzwirtschaftliches demand management zeitige nur elnen allokativen und keinen aggregativen Effekt: Durch eine Erhohung der Transformationsausgaben zur Anhebung des Gesamtausgabenniveaus verdränge der staat im gleichen Zug private Ausgaben. Im Ergebnis ändere sich nur die Struktur und nicht das Niveau der gesamtwirtschaftichen Ausgaben. "Monetarists ... generally assert that government spending financed by either taxing or borrowing from the public is mainly a resource transfer from the private sector to the government, with little net effect on total spending" 3 ? Dieser Tatbestand, daB expansive finanzwirtschaftliche Nachfrageimpulse private Ausgaben zurückdrängen, wurde von Spencer und Yohe ${ }^{4)}$ in Anlehnung an Culbertson mit dem Etikett "crowding-out" belegt. Popularisiert wurde dieser Begriff hauptsächlich durch die beiden Aufsätze "The

1) Vgl. Friedman, M., Capitalism and Freedom, Chicago, 1962, insbesondere S. $75 \mathrm{ff.;}$ Friedman, M., Meiselman, D., The Relative Stability of Monetary Velocity and the Investment Multiplier in the United States, 1897-1958, in: Commission on Money and Credit (Hrsg.), Englewood Cliffs, N.J., Prentice-Hall, 1963 , S. 163 ff.; Friedman, M., Heller, W.W., Monetary vs. Fiscal Policy. A Dialogue, New York, 1969.

2) Vgl. Insbesondere Andersen, L.C., Jordan, J.L., Monetary and Fiscal Actions: A Test of Their Relative Importance in Economic Stabilization, in: Federal Reserve Bank of St. Louis Review, November 1969, S. 5 ff.

3) Spencer, R.W., Yohe, W.P., The "Crowding-Out" of Private Expenditures by Fiscal Policy Actions, in: Federal Reserve Bank of St. Louis Review, Oktober 1970, wiederabgedruckt in: Boorman, J.T., Havrilesky, T.M., Money Supply, Money Demand and Macroeconomic Models, Northbrook, 1972, S. 431.

4) Vgl. ebenda, S. 430 . 
'Crowding-out' of Private Expenditures by Fiscal Policy Actions"1) von Spencer und Yohe im Jahr 1970 sowie "'Crowding-out' and its Critics"2) von Carlson und Spencer im Jahr 1975. Das Pendant zum crowding-out, also die Initiierung privater Ausgaben durch finanzwirtschaftliche AnkurbelungsmaBnahmen, woran Keynesianer weiterhin festhalten, wird bisweilen als "pulling-in"3) oder auch als "crowding-in"4) bezeichnet.

In der Bundesrepublik Deutschland hat sich die von den Monetaristen angeregte Diskussion der über einen langen Zeitraum als gesichert geltenden keynesianischen GesetzmäBigkeit in der letzten zeit erheblich intensiviert, insbesondere, als es trotz massiver Anwendung der keynesianischen Rezeptur nicht gelang, den scharfen Konjunktureinbruch im Jahr $1975 \mathrm{zu}$ verhindern. Mit der vorliegenden Arbeit soll zu dieser Debatte, die sich sowohl auf empirischer als auch auf theoretischer Ebene bewegt, ein Beitrag geliefert werden. Es wird untersucht, wie sich die These der Monetaristen vom crowding-out theoretisch begründen läBt, oder anders gesehen: wie man die These der Keynesianer von der aggregativen wirksamkeit eines expansiven finanzwirtschaftlichen demand management kausallogisch falsifizieren kann. Zu diesem Zweck werden die crowding-out-Effekte, mit Hilfe deren erklärt werden kann, "daB im Ergebnis dem Mehr an staatlichen Leistungen ein

1) Spencer, R.W., Yohe, W.P., The "Crowding-Out" of Private Expenditures by Fiscal Policy Actions, a.a.O., S. 430 .

2) Carlson, M.K., Spencer, R.W., Crowding-Out and its Critics, in: Federal Reserve Bank of St. Louis Review, Dezember 1975, S. 2 ff.

3) Vgl. z.B. Hendershott, P.H., A Tax Cut in a Multiple Security Model: Crowding-Out, Pulling-In and the Term Structure of Interest Rates, in: Journal. of Finance, 1976 , S. 1185 ff.

4) Vgl. z.B. Friedman, B., Crowding-Out or Crowding-In? Economic Consequences of Financing Government Deficits, in: Brookings Papers on Economic Activity, 1978 , S. $593 \mathrm{ff}$. 
Weniger an privaten Ausgaben gegenübersteht" ${ }^{1)}$ präsentiert und auf ihre plausibilität hin überprüft.

\section{B. Aufbau der Arbeit}

Die Arbeit besteht aus zwei Teilen. Davon ist der zweite der wichtigere. Hier wird eine Antwort auf die Frage gesucht, wie eine Verminderung der von den Privaten entfalteten Güternachfrage infolge eines expansiven finanzwirtschaftlichen demand management begründet werden kann. Um Vorarbeiten für diese Analyse geht es im ersten Teil, verbunden mit einigen allgemeinen Ausführungen zum crowdingout. So werden zunächst (im zweiten Kapitel) die dogmenhistorischen wurzeln der crowding-out-These aufgezeigt und (im dritten Kapitel) mögliche Formen des crowding-out abgehandelt. Im vierten Kapitel wird dann der Untersuchungsrahmen für die nachfolgende crowding-out-Analyse eingegrenzt und ein Grundmodell vom IS-LM-TYp präsentiert.

Der zweite Teil ist in sechs Kapitel untergliedert, wobei die ersten fünf jeweils einem der fünf wesentlichen Transmissionswege finanzwirtschaftlicher Nachfrageimpulse gewidmet sind. Zunächst geht es um einen Finanzierungseffekt. Gesamtwirtschaftlich gesehen kann bei einem konstanten Geldangebot eine infolge des staatlichen Handelns vermehrte gesamtwirtschaftliche Güternachfrage nur durch eine Beschleunigung des Geldumlaufs finanziert werden. Durch den Anstieg des Zinsniveaus, welcher dafür sorgt, werden die zinsabhängigen Ausgaben der Privaten zurückgedrängt.

Im zweiten Kapitel wird der Frage nachgegangen, ob staatliche Ankurbelungsmaßnahmen aufgrund von Vermögenseffekten an Effizienz einbüBen. Bei Vermögenseffekten werden finanzwirtschaftliche Impulse via Veränderungen des privaten Vermögens auf die Ausgabenaktivitäten der Privaten übertragen. Im Vordergrund

1) Sachverständigenrat zur Begutachtung der gesamtwirtschaftlichen Entwicklung, Jahresgutachten 1978/79, Bundestagsdrucksache $8 / 2314$, Ziff. 484 . 
stehen "konventionelle" Vermögenseffekte, die Folge einer mengenmäBigen Variation der von den Privaten gehaltenen Staatsschulden sind. Als Exkurs wird auf die von Stützel angeregte Diskussion eingegangen, ob der Kapitalmarkt mehr elner "Quelle" oder einer "Fontäne" ähnelt.

Vergleichsweise selten werden in die crowding-out-Analyse die Staatsausgaben einbezogen, welche die privaten Aktivitäten anregen sollen. Fallen die dadurch bewirkten pulling-in-Effekte schwach aus, kann damit ebenso wie mit Verdrängungseffekten begründet werden, daB infolge einer finanzwirtschaftlichen MaBnahme die privaten Ausgaben unter den Betrag sinken, welcher bel Abwesenheit der staatlichen Intervention getätigt worden wäre. Die Gründe, welche dafür sprechen, daB Staatsausgabenimpulse, für sich genommen, die privaten Aktivitäten nur wenig oder gar nicht stimulieren, sind Gegenstand des dritten Kapitels.

Im vierten Kapitel wird die Annahme eines konstanten Preisniveaus aufgehoben. Bei einem variablen Preisniveau muB explizit auf die realen Privatausgaben abgestellt werden. Diese sinken nicht nur dann, wenn die nominellen Privatausgaben abnehmen, sondern auch, wenn es zu einer Preisniveauerhöhung kommt. Einen Anstieg des Preisniveaus können finanzwirtschaftliche Nachfrageimpulse auf den Wegen des Nachfragesogs und des Angebotsdrucks bewirken; beide Wege werden abgehandelt.

Im fünften Kapitel geht es um Erwartungseffekte. Das Verhalten der Privaten wird nicht zuletzt durch ihre zukunftseinschätzung bestimmt, und diese kann durch finanzwirtschaftliche Aktivitäten so beeinfluBt werden, daß es zu einer Verminderung der gegenwärtig von ihnen entfalteten Güternachfrage kommt. 
Im sechsten Kapitel werden die Ergebnisse der Wirkungsanalyse zusammengefaBt.

Zweites Kap1tel: Dogmenhistorische Wurzeln der crowding-out-These

\section{A. Die crowding-out-These als Bestandteil der klassischen Theorie}

Die crowding-out-These ist keineswegs neu. Sie war schon Bestandtell der klassischen Lehre und basierte auf dem Sayschen Theorem (Théorie des débouchés) sowie auf der Annahme flexibler Löhne ${ }^{1 !}$.

Das Saysche Theorem ${ }^{2)}$ besagt, daB sich jedes Güterangebot seine eigene Nachfrage schafft. Es wurde von den Klassikern wie folgt begründet: Im Zuge der Produktion von Gutern entsteht Einkommen in gleicher Höhe für die dabei mitwirkenden Produktionsfaktoren. Die Teile des Einkommens, welche gespart, also nicht zum Kauf von Konsumgütern verwendet werden, führen $z u$ keinem Nachfrageausfall, sondern lediglich zu elner Nachfrageverschiebung. Denn die sparer geben am Kreditmarkt ihre nicht konsumtiv verwendeten Einkommensteile in voller Höhe an andere Wirtschaftseinheiten, welche damit Investitions-

1) Vgl. hierzu Landmann, 0., Keynes in der heutigen Wirtschaftstheorie, a.a.O., S. $137 \mathrm{ff}$. und Spencer, R.W., Yohe, W.P., The "Crowding Out" of Private Expenditures by Fiscal Policy Actions, a.a.0., s. $433 \mathrm{ff}$. sowie Neumark, F., Grundsätze und Arten der Haushaltsführung und Finanzbedarfsdeckung, in: Gerloff, W., Neumark, F. (Hrsg.), Handbuch der Finanzwissenschaft, 1. Band, Tübingen, $1952^{2}$, S. $610 \mathrm{ff}$.

2) Vgl. Say, J.B., Traité d'économie politique, Tome I, Paris $1826^{5}$, insbesondere Kapitel XI; umfassend informiert Sowell, T., Say's Law. An Historical Analysis, Princeton, N.J., 1972 . 
ausgaben tätigen ${ }^{1)}$. Mithin wird das gesamtwirtschaftliche Güterangebot vollständig durch die gesamtwirtschaftliche Güternachfrage absorbiert; jedes Angebot an Gütern schafft sich folglich selbst die zum Absatz notwendige Nachfrage.

Das Saysche Theorem beruht einmal auf der Annahme, Geld werde ausschlieBlich als "aktive Kasse" (Transaktionskasse) und nicht auch aus dem spekulationsmotiv in Form von "idle money" gehalten, und zum anderen auf der Annahme, der zins sei flexibel.

Dem Geld wurde von den Klassikern deshalb nur eine Tauschmittelfunktion zugestanden, weil es nach ihrer Einschätzung ökonomisch widersinnig war, freiwillig auf eine zinsbringende Anlage auf den Finanzmärkten zu verzichten und stattdessen Ersparnisse in Form von zinsloser Spekulationskasse zu horten. "The sole use of money is to circulate consumable goods" 2 ! Zinsreduktionen lösen dann bei den Sparern nicht den Wunsch aus, Einkommensteile lieber als liquide Mittel zu halten, anstatt sie am Kreditmarkt den Investoren anzubieten. In der Sprache der Quantitätsgleichung ausgedrückt: Die Zinselastizität der Kassenhaltung ist gleich Null (Cambridge-Gleichung) bzw. die Umlaufsgeschwindigkeit des Geldes ist zinsunabhängig (Fishersche Gleichung) - oder mit Blick auf den Gütermarkt: Da es kein Horten von Geld gibt, ist das Sparen mit dem Kreditangebot identisch; die nicht konsumierten Einkommens-

1) "That portion which he annually saves, as for the sake of the profit, it is immediately employed as capital, is consumed in the same manner, and nearly in the same time too, but by a different set of people.". Smith, A., An Inquiry into the Nature and the Causes of the Wealth of Nations, London, 1776, Campbell, R.H., Skinner, A.S. (Hrsg.), vol. 1, Oxford, 1976, S. 338.

2) Smith, A., An Inquiry into the Nature and the Causes of the Wealth of Nations, a.a.O., S. 339 . 
teile stehen vollständig für die Finanzierung von Investitionen zur Verfügung.

Die Annahme des flexiblen Zinses stellt sicher, daB der Kreditmarkt "geräumt" wird und damit Sparen und Investieren zum Ausgleich gebracht werden. Der Zins steuert also die Pläne der Privaten so, daB dle Konsumenten den Investoren genau so viel vom Güterangebot überlassen, wie diese nachfrag̣en.

DaB dieses Güterangebot auch jenes ist, bel dem alle Arbeitswilligen beschaftigt sind, besorgt die zweite der beiden Annahmen, mit denen die Klassiker ein crowding-out begründet haben: die Hypothese flexibler Löhne. Bei Arbeitslosigkeit entsteht nämlich ein Druck auf die Lorhne, wodurch mehr Produktion rentabel wird, und zusätzliche Arbeitskrăfe eingestellt werden. Diese Lohnsenkungstendenz hält solange an, bis Vollbeschäftigung herrscht.

Die Konsequenz der von den Klassikern angestellten Uberlegungen ist, daB sich ein finanzwirtschaftliches demand management erübrigt. Der private sektor tendlert nămlich von sich aus zur Vollbeschäftigung. Wenn zusätzliche Güternachfrage vom staat entfaltet wird, hat dies ein crowdingout zur Folge. Uber welche Wege unter den Annahmen der Klassiker die privaten Ausgaben durch das staatliche Handeln verdrängt werden, hängt von der Finanzlerung der zusätzlichen staatsausgaben ab.

Sowohl im Fall der Steuer- als auch der Kreditmarktfinanzierung bleibt die Geldmenge konstant; weil zudem die Umlaufsgeschwindigkeit des Geldes durch die finanzpolitische Aktivität nicht beeinfluBt wird, können die monetären Gesamtausgaben - trotz vermehrter staatlicher Güternachfrage - nicht steigen, und sinken die monetären 
Ausgaben der Privaten um den Betrag der Zusatznachfrage des Staates.

Bei der Steuerfinanzierung können folglich die Privaten ihren Verpflichtungen nicht durch Auflösung von "idle money" nachkommen, sondern müssen ihre Ausgaben einschränken. Uber die Struktur der verdrängten privaten Güternachfrage entscheidet die Art der steuer: Verbrauchsteuern beeinträchtigen vor allem den privaten Konsum, Gewinnsteuern die privaten Investitionen.

Beschafft sich der Staat die Mittel auf dem Weg einer Kreditaufnahme bei den Privaten, erfolgt das crowdingout über einen zinseffekt. Durch den erhöhten Mittelbedarf des staates entsteht eine zinssteigerungstendenz, und die privaten zinsabhängigen Ausgaben werden zurückgedrängt. Insoweit die Privaten zinsinduziert vermehrt sparen, gehen die privaten Konsumausgaben zurück; insoweit der Staat auch ansonsten getätigte private Ersparnis absorbiert, fallen private Investitionsausgaben aus.

Wenn sich der Staat zur Finanzierung seiner Ausgaben einer Kreditaufnahme bei der Notenbank bedient, treten im privaten Sektor keine monetären Entzugseffekte auf. Es gelingt dem Staat deshalb in der Tat, das monetäre Gesamtausgabenniveau $\mathrm{zu}$ heben. Dennoch verdrängt er private Güternachfrage, jetzt aber nicht nominal, sondern real. Denn bei Vollbeschäftigung kann der staat nur dann über mehr Güter verfügen, wenn andere - die Privaten - dafür zurückstehen. Als Verdrăngungsvariable dient das Preisniveau. Setzt der Staat seine realen Ausgabenpläne durch, so erhöht sich im Zuge der Nachfragekonkurrenz auf dem Gütermarkt das Preisniveau solange, bis die realen Ausgaben der Privaten um den Betrag der realen zusatznachfrage des staates abgenommen haben, und die 
Ausgabenpläne von Staat und den Privaten miteinander kompatibel sind ${ }^{1}$ !

B. Negierung der crowding-out-These in der keynesianischen Theorie

Durch die Weltwirtschaftskrise zwischen 1929 und 1932 wurde das klassische Credo zutiefst erschüttert. Die Kluft zwischen klassischer Beschätigungstheorie und ökonomischer Realität versuchte Keynes mit seinem 1936 erschienenen Werk "The General Theory of Employment, Interest and Money" zu schließen.

Den Kern der Keynesschen Kritik an den Klassikern sowie seiner Diagnose von Unterbeschäftigungssituationen bildeten zum einen die Berücksichtigung des Spekulationsmotivs als Bestimmungsgrund der Geldnachfrage und zum anderen die Annahme von nach unten starren Löhnen. Während mit Hilfe der keynesianischen Geldnachfragefunktion

1) Für die Klassiker verbot sich eine Erhöhung der staatlichen Güternachfrage nicht nur wegen der erwähnten aggregativen, sondern auch wegen folgender allokativer Ineffizienz. Sie hielten staatliches Handeln fur ziemlich unproduktiv. Mithin würde in dem MaBe, wie im privaten Sektor produktiv verwendete Ressourcen in den öffentlichen Bereich gelängen, das Wachstum des Produktionspotentials geschmälert. Ganz anderer Meinung waren diesbezüglich die Merkantilisten. In ihren Schriften findet sich "die Auffassung .... den öffentlichen Ausgaben, insbesondere denen zur Förderung gewisser Wirtschaftszweige, komme eine so groBe Produktivität zu, daB hohe Ausgaben aus volkswirtschaftlichen Gründen prinzipiell erwünscht seien ...". Neumark, F., Grundsätze und Arten der Haushaltsführung und Finanzbedarfsdeckung, a.a.0., S. 610. Scharfe Kritiker der klassischen These von der weitgehenden Unproduktivität öffentlicher Ausgaben waren auch einige Finanzwissenschaftler, welche heute unter dem Begriff "Historische Schule" zusammengefaBt werden. Vgl. z.B. Dietzel, C., Das System der Staatsanleihen im Zusammenhang der Volkswirtschaft betrachtet, Heidelberg, 1855, in: Diehl, K., Mombert, P. (Hrsg.), Ausgewählte Lesestücke zum Studium der politischen ơkonomie, Neuedition von Hickel, R., Frankfurt, Berlin, Wien, 1980, S. 211 ff. 
Unterbeschäftigungslagen insoweit erklärt werden können, wie diese auf eine deflatorische Lücke, also auf eine Differenz zwischen Güternachfrage und Güterangebot zurückzuführen sind, macht die Annahme von nach unten nicht beweglichen Lobnen auch sogenannte Unterbeschäftigungsgleichgewichte plausibel; derartige situationen sind gekennzeichnet durch Unterbeschäfigung auf dem Arbeitsmarkt, obgleich auf dem Gütermarkt Nachfrage und Angebot übereinstimmen ${ }^{1 !}$.

Worauf es bei der keynesianischen Geldnachfragefunktion ankommt, ist djee Tatsache, daB es angesichts von Unsicherheit bezüglich der Kursentwicklung von Forderungsrechten durchaus sinnvoll sein kann, anstelle des Kaufs solcher Papiere, Ersparnisse lieber in Form von nicht verzinslichem aber dafür kurssicherem Geld anzulegen und zwar dann, wenn Kursverluste zu erwarten sind und diese den $\mathrm{Z}$ insertrag überkompensieren. Geld wird mithin nicht nur einkommensabhängig aus dem (klassischen) Transaktionsmotiv, sondern auch zinsabhängig aus dem (keynesianischen) Spekulationsmotiv nachgefragt. Für das Gütermarktgleichgewicht bedeutet diese dem Geld zugestandene Wertaufbewahrungsfunktion die Negierung des von den Klassikern abgeleiteten Konnex zwischen Sparen und Investieren und damit die $\mathrm{Ab}-$ lehnung des Sayschen Theorems. Sparen ist nun nicht mehr Nachfrageverlagerung, sondern erst einmal Nachfrageausfall2) Ob die Ersparnisse an anderer Stelle wieder via Investitionsguternachfrage in den Kreislauf injiziert werden und eine deflatorische Lucke verhindert wird, hängt davon $a b$, welcher Marktzins sich aus der Liquiditătspraferenz der Wirtschaftssubjekte und dem Geld-

1) Vgl. Keynes, J.M., The General Theory of Employment, Interest and Money, London, 1936, S. 3 ff. sowie Landmann, 0., Keynes in der heutigen Wirtschaftstheorie, a.a.0., S. 137 ff.

2) "An act of individual saving means - so to speak - a decision not to have dinner to-day. But it does not necessitate a decision to have dinner or to buy a pair of boots a week hence or a year hence or to consume any specified thing at any specified date". Keynes, J.M., The General Theory of Employment, Interest and Money, a.a.0., S. 210 . 
angebot ergibt, und welcher Investitionsumfang den Unternehmern bei diesem Marktzins lohnend erscheint.

Doch auch bei einem Gütermarktglelchgewicht ist keineswegs sichergestellt, daB bel dem entsprechenden Produktionsniveau alle Arbeitswilligen auch beschäftigt sind und zwar deswegen nicht, weil nach Ansicht von Keynes die Löhne nach unten starr sind. Eine Tendenz zur Beseitigung dieses Unterbeschäftigungsgleichgewichts durch eine Verringerung der Löhne, was von den Klassikern erwartet wurde, entsteht in diesem Fall nicht.

Für beide Unterbeschäftigungsursachen empfahl Keynes dieselbe Therapie: Justierung der gesamtwirtschaftlichen Ausgaben vor allem mittels staatlicher Güternachfrage auf genau das Niveau, welches Vollbeschäftigung aller Arbeitskräfte ermöglicht. DaB mit einem expansiven finanzwirtschaftlichen demand management die intendierte Wirkung auch erreicht wird, stand für Keynes nicht in Frage. Insbesondere aus den folgenden zwei Gründen erschien ihm seine Politikempfehlung erfolgversprechend.

Zum einen regen zusätzliche Transformationsausgaben private Ausgaben an und erzeugen ein Mehrfaches ihrer selbst an gesamtwirtschaftlicher Güternachfrage. Denn die Güternachfrage des Staates läßt an der Stelle ihrer Produktion Einkommen bei den dort Beschäftigten entstehen, was nach Maßgabe der marginalen Konsumquote die private Güternachfrage vermehrt und $z u$ einem sich weiter fortpflanzenden Anstieg von Einkommen und privaten Ausgaben führt. Eine Ausdehnung der Güternachfrage des Staates hat mithin eine multiplikativ vergrößerte Wirkung auf die gesamtwirtschaftliche Güternachfrage, so daB der staat selbst nur insoweit die lücke zwischen aktueller und mit Vollbeschäftigung konformer Güternachfrage schlieBen muB, 
wie dies nicht durch die erwarteten Mehrausgaben der Privaten geschieht.

Zum anderen wirkt sich eine deflatorische Lücke darin aus, daB die Wirtschaftssubjekte ihre Kassenhaltung aufstocken und gesparte Einkommensteile nicht an Investoren zedieren. Dann aber steht ein hinreichendes Aktivierungspotential an "idle money" zur Verfügung, und zusätzliche Güternachfrage kann, gesamtwirtschaftlich gesehen, durch eine Beschleunigung des Geldumlaufs finanziert werden.

War Keynes auch von der Effizienz eines finanzwirtschaftlichen demand management zur Beseitigung von Unterbeschäftigung uberzeugt, so werden in der "General Theory of Employment, Interest and Money" am Rande dennoch Gründe erwähnt, durch welche die finanzwirtschaftlichen Stabilisierungsmaßnahmen Wirksamkeit verlieren können ${ }^{1)}$ ?

Erstens schloB Keynes nicht aus, daB zur Aktivierung von "idle money" Zinserhöhungen nötig sind, also der Geldumlauf nur durch eine fühlbare $\mathrm{Z}$ inssteigerung beschleunigt werden kann, und zinsabhängige Ausgaben der Privaten zurückgedrängt werden ${ }^{2}$ !

Zweitens erwähnte Keynes Erwartungseffekte, die auf einen mit staatlicher Kreditfinanzierung zusammenhängenden Vertrauensschwund bei den Privaten zurückzuführen sind und sich in einem Ausfall von privater Investitionsgüternachfrage äuBern: Investitionspläne werden deshalb vertagt, weil der Verlust an Zukunftsvertrauen die Liquiditäts-

1) Vgl. Keynes, J.M., The General Theory of Employment, Interest and Money, a.a.O., S. $119 \mathrm{ff.;}$ vgl. auch Spencer, R.W., Yohe, W.P., The "Crowding Out" of Private Expenditures by Fiscal Policy Actions, a.a.0., s. $436 \mathrm{ff}$.

2) "The method of financing the policy and the increased working cash, required by the increased employment and the associated rise of prices, may have the effect of increasing the interest and so retarding investment in other directions ...". Keynes, J.M., The General Theory of Employment, Interest and Money, a.a.O., S. 119; vgl. auch S. 55 ff. unten. 
präferenz und dadurch das zinsniveau erhöht, und weil der Vertrauensschwund $z u$ einem Abschlag bei der Grenzleistungsfähigkeit des Kapitals fuhrt ${ }^{1}$ !

Drittens wies Keynes darauf hin, da in einer offenen Volkswirtschaft finanzwirtschaftliche Impulse in dem MaBe in das Ausland versickern, wie sich dadurch der Leistungsbilanzsaldo verschlechtert.

Weil viertens nach Ansicht von Keynes das psychologische Gesetz vom Konsum gilt, ist der Gesamtausgabenmultiplikator einer bestimmten Ausdehnung der staatlichen Güternachfrage um so geringer, je höher das Volkseinkommen in der Ausgangslage schon ist, und der Gesamtausgabenmultiplikator in einer bestimmten Ausgangslage um so kleiner, je stärker die Vermehrung der Transformationsausgaben ausfällt. Zudem verringert sich die Grenzneigung zum Konsum im Aufschwung deswegen, weil der Gewinneinkommensanteil steigt und die Gewinneinkommensbezieher eine unterdurchschnittliche Konsumquote haben und weil das vorherige Entsparen von dann nicht mehr Arbeitslosen entfällt.

Zusammengenommen: Keynes war sich zwar der Möglichkeit bewuBt, daB im Zuge staatlicher AnkurbelungsmaBnahmen auch crowding-out-Effekte auftreten können; er schätzte jedoch ihre stärke bei weitem nicht so ein, wie dies von den Klassikern getan wurde und heute wieder von den Monetaristen getan wird.

1) Vgl. S. $158 \mathrm{ff}$. unten. 
C. Renaissance der crowding-out-These in der monetaristischen Theorie

Bis Ende der sechziger Jahre blieben die keynesianischen GesetzmäBigkeiten hinsichtlich der stabilisierungspolitischen Wirksamkeit eines finanzwirtschaftlichen demand management zur Beseitigung von Unterbeschäftigung im wesentlichen unbestritten. Erst mit der "monetarist counter-revolution"1) erlebte das crowdingout-Postulat der Klassiker eine Renaissance.

In der ersten Phase der sich daran anschlieBenden und sich in den letzten Jahren intensivierenden Diskussion ${ }^{2}$ ) ging es darum, daB infolge des staatlichen Handelns der Bedarf an Transaktionskasse steigt, und dieser bei einem konstanten Geldangebot nur über einen Abbau der Spekulationskasse befriedigt werden kann. In dieser sicht hängt der Umfang eines crowding-out davon ab, wie stark sich die Zinsen erhöhen müssen, um die Halter

1) Johnson, H.G., The Keynesian Revolution and the Monetarist Counter-Revolution, in: The American Economic Review, Papers and Proceedings, Mai 1971, S. 1.

2) Vgl. für einen Uberblick Paul, E., Der Crowding-OutEffect, in: Wirtschaftswissenschaftliches Studium, 1977, S. $177 \mathrm{ff.;}$ Alexander, V., Brückmann, F., Crowding-outEffekte kommunaler Schuldenpolitik in der Bundesrepublik Deutschland, in: Zeitschrift für Wirtschafts- und Sozialwissenschaften, 1978, S. $214 \mathrm{ff.;} \mathrm{Argy,} \mathrm{V.,} \mathrm{Some} \mathrm{Notes}$ on Fiscal Policy and Crowding Out, in: Australian Economic Review, 1979, S. 64 ff.; Arcelli, M., Valiani, R., Crowding Out: Some Reflections on Economic Policy, in: Review of the Economic Conditions in Italy, Nr. 2, 1979, S. $189 \mathrm{ff.;}$ Mackscheidt, K., Crowding-Out als MaBstab für die Effizienz der fiscal policy?, in: Duwendag, D., Siebert, H. (Hrsg.), Politik und Markt, Wirtschaftspolitische Probleme der $80 \mathrm{er}$ Jahre, stuttgart, New York, 1980, S. 53 ff.; Dieckheuer, G., Der Crowdingout-Effekt - zum gegenwärtigen Stand von Theorie und Empirie, in: Vierteljahreshefte zur Wirtschaftsforschung, 1980, S. 126 ff.; Westphal, U., Empirische Aspekte des Crowding-out, in: Ehrlicher, w. (Hrsg.), Geldpolitik, zins und Staatsverschuldung, Schriften des Vereins für Socialpolitik, N.F. 111, Berlin 1981, S. $209 \mathrm{ff}$.; Koch, W.A.S., Lang, E., Crowding-out und staatliches debt management, in: WSI-Mitteilungen, 1981, S. $50 \mathrm{ff}$. 
von Spekulationskasse zu bewegen, den zur Finanzierung der zusätzlichen Ausgaben nötigen Geldbetrag freizugeben. Im Mittelpunkt ${ }^{1)}$ stand mithin erneut der Streit um die zinselastizität der Geldnachfrage.

Durch Milton Friedman angeregt ${ }^{2)}$, der darauf hinwies, daB es fur ein crowding-out nicht nur auf die $\mathrm{zins-}$, sondern auch auf die Vermögenselastizität der Geldnachfrage (vor allem in mittlerer Frist) ankommt, verlagerte sich die Diskussion in der zweiten Phase auf "Wealth Effects and the Effectiveness of ... Fiscal Policies" ${ }^{3}$ ?

In der dritten und noch andauernden phase ist zum einen das spektrum der crowding-out-Effekte erweitert worden. Zum anderen sind auch pulling-in-Effekte in die crowdingout-Diskussion einbezogen worden. Finanzwirtschaftliche MaBnahmen und die Ausgaben der Privaten sind nämlich über eine Vielzahl von Transmissionskanälen miteinander verknüpft. Wenn infolge finanzwirtschaftlicher Impulse die Privaten ihre Ausgaben variieren, so ist dies das Ergebnis von gegenläufigen, partiellen Expansions- und Kontraktionseffekten. Ob der Gesamteffekt die Qualität eines pulling-in oder eines crowding-out hat, hängt von der relativen stärke der einzelnen Anreiz- und Dämpfungsfaktoren ab. Die Gründe dafür, daB ein expansives finanzwirtschaftliches demand management aggregativ unwirksam ist, brauchen mithin nicht nur bei den Verdrängungseffekten zu liegen; sie sind auch

1) Vgl. z.B. Kaminow, J., The Myth of Fiscal Pollcy: The Monetarist View, in: Federal Reserve Bank of Philadelphia Business Review, Dezember 1969, S. $10 \mathrm{ff}$. sowie Tobin, J., Friedman's Theoretical Framework, in: Gordon, R.J. (Hrsg.), Milton Friedman's Monetary Framework, A Debate with His Critics, Chicago, London,1974, S. $77 \mathrm{f}$.

2) Vgl. Friedman, M., Comments on the Critics, in: Gordon, R.J. (Hrsg.), Milton Friedman's Monetary Framework. A Debate with His Critics, Chicago, London, 1974, S. $137 \mathrm{ff}$.

3) Meyer, L.H., Wealth Effects and the Effectiveness of Monetary and Fiscal Policies, in: Journal of Money, Credit, and Banking, 1974, S. 481. 
darin zu suchen, daB die Injektionseffekte schwach ausfallen und die Verdrängungseffekte deshalb die Oberhand behalten ${ }^{1}$ ?

3. Kapitel: Formen des crowdinq-out

A. Hauptelemente des Ablaufschemas finanzwirtschaftlicher Maßnahmen

In der Literatur werden verschiedene Formen des crowdingout unterschieden. Diese Formen lassen sich gut anhand der drei Hauptelemente des Ablaufschemas einer finanzwirtschaftlichen Maßnahme (Impuls, Transmission, Effekt) erläutern und zu Gruppen zusammenfassen.

Die Veränderung einer ökonomischen Variablen 1st ein Impuls, die Auswirkung davon auf eine andere ökonomische Variable ein Effekt. Es gibt Primärimpulse und Sekundärimpulse sowie Zwischeneffekte und Endeffekte. Primärimpulse sind die Veränderungen von wirtschaftspolitischen Parametern, hier also die finanzwirtschaftliche MaBnahme. Auf die Endeffektvariablen, und das sind hier die privaten Ausgaben, werden die finanzwirtschaftlichen Impulse entweder direkt oder indirekt übertragen. Bei einer direkten Transmission sind die Primärimpulsvariablen unmittelbar Bestimmungsgründe der Endeffektvariablen; bei einer indirekten Ubertragung sind Primärimpuls- und Endeffektvariable nicht direkt, sondern nur mittelbar durch Transmissionsvariablen verknüpft. Durch das staatliche Handeln induzierte Anderungen von Transmissionsvariablen sind zugleich Sekundärimpulse und Zwischeneffekte.

1) Vgl. z.B. Artis, M.J., Fiscal Policy and Crowding out, in: Posner, M. (Hrsg.), Demand Management, London, 1978 , S. 173. 
B. Impuls: Direktes und indirektes crowding-out

Bei der Unterscheldung zwischen direktem und indirektem crowding-out wird an die beiden Arten, wie finanzwirtschaftliche Primärimpulse in den privaten Aktivitätsbereich ubertragen werden können, angeknüpft ${ }^{1}$ !

Im Fall eines direkten crowding-out sinkt die private Güternachfrage, ohne daB Transmissionsvariablen berührt werden, weil Primärimpulsvariablen unmittelbar Bestimmungsgründe der privaten Güternachfrage sind. Erhöht z.B. der staat seine Transformationsausgaben und sehen die Privaten die damit bereitgestellten öffentlichen Güter als Substitute zu privaten Gütern an, dann werden durch die staatlichen Leistungen die Privaten dazu veranlaßt, ihre eigene Güternachfrage einzuschränken.

Ein indirektes crowding-out vollzieht sich dagegen über Sekundärimpulse. Die Privaten schränken ihre Ausgaben nicht deshalb ein, weil der Staat Politikvariablen verändert, sondern, weil infolge dieser Maßnahme Transmissionsvariablen, wie Zinsen und Preise, andere Werte annehmen.

C. Transmission

1. Ex ante und ex post crowding-out

Im Hinblick auf den Zeitpunkt, zu dem ein crowding-outImpuls in den privaten Aktivitätsbereich übertragen wird, kann in Anlehnung an die Terminoloqie von David

1) Diese Klassifikation geht zurück auf Buiter, W., "Crowding out" and the Effectiveness of Fiscal Policy, in: Journal of Public Economics, 1977, S. 311 ff.; alternativ finden noch das Begriffspaar "strukturelles" (=indirektes) und "reduzierte Form" (= direktes) crowding-out sowie das in unserer Analyse mit einem anderen Inhalt belegte Begriffispaar "ex post" (=indirektes) und "ex ante" " ( = direktes) crowding-out Anwendung filler-978-3-631-75197-8 
und scadding ex ante crowding-out von ex post crowdingout unterschieden werden ${ }^{1 !}$. MaBgebend für diese Einteilung ist, ob der Verdrängungseffekt dem finanzwirtschaftlichen Handeln zeitlich vor-oder nachgelagert 1st. Unter ex post crowding-out wird der Teil der Verdrängung subsumiert, welcher eintritt, nachdem mit finanzwirtschaftlichen Aktivităten auf die privaten Guter- und Geldströme eingewirkt worden ist. Sinken die privaten Ausgaben indessen schon, bevor der staat eine Maßnahme ergriffen hat, sind also Ankündigungseffekte wirksam, sprechen wir von ex ante crowding-out.

2. Kurzfristiges, mittelfristiges und langfristiges crowding-out

Eine andere Art der zeitlichen Differenzierung stellt die Unterscheidung in kurzfristiges, mittelfristiges und langfristiges crowding-out dar 2 ! sie findet hauptsächlich dort Anwendung, wo konventionel.le Vermögenseffekte in die crowding-out-Analyse einbezogen werden. Solche Vermögenseffekte resultieren aus einer Erhöhung des Staatsschuldenstandes in den Portefeuilles der Privaten.

Werden die privaten Ausgaben schon in der sogenannten Entstehungsphase des Defizits ${ }^{3 l}$ verdrängt, dann liegt

1) Vgl. David, P.A., Scadding, J.L., Private Savings: Ultrarationality, Aggregation, and "E€nision's Iaw", in: Journal of Political Economy, 1974, S. $243 \mathrm{ff.;}$ wie in der Fußnote 1) auf seite 19 erwähnt, sind in der Begriffsfassung von David und scadding ex ante und ex post crowding-out synonyma für direktes und indirektes crowding-out.

2) Vgl. Friedman, M., Comments on the Critics, a.a.o., S. $140 \mathrm{ff}$. Sowie Rose, M., Finanzwissenschaftliche Makrotheorie, München, 1980, S. 358 .

3) Vgl. zu dem Begriffspaar Entstehungsphase des Defizits und reine Finanzierungsphase des Defizits: Dieckheuer, G., Möglichkeiten und Risiken einer kreditfinanzierten Stabilisierungspolitik, in: Wirtschaftsdienst, 1977, S. 559 . 
ein kurzfristiges crowding-out (first-round crowdingout) ${ }^{1)}$ vor. Die Entstehungsphase des Defizits ist der Ze1traum, in dem alle Anpassungsvorgänge auf den Güterund Finanzmärkten abgeschlossen werden, welche beispielsweise durch eine kreditmarktfinanzierte staatsausgabenerhöhung ausgelöst worden sind. In Wirkungsanalysen wird zumeist angenommen, die finanzwirtschaftlichen Politikvariablen wirden zu Beginn einer Haushaltsperiode verändert und dile Anpassungsprozesse noch innerhalb der Haushaltsperiode enden, weshalb die Entstehungsphase des Defizits mit einer Haushaltsperiode gleichgesetzt wird.

Die Ausdehnung der staatlichen Ausgaben darf in den der Entstehungsphase des Defizits folgenden Haushaltsperioden, der sogenannten reinen Finanzierungsphase des Defizits, nicht rückgängig gemacht werden, weil ansonsten von seiten der Staatsausgaben dämpfende Einflüsse auf die private Güternachfrage entfaltet würden. Dazu ist es nötig, solange und soweit induzierte steuereinnahmen nicht hinreichen, in jeder Haushaltsperiode erneut staatspapiere im privaten Sektor zu plazieren. In der reinen Finanzierungsphase des Defizits steigt also ständig der Bestand an Staatsschulden in den Portefeuilles der Privaten. Weil damit Portfolioumstrukturierungen einhergehen, im Zuge deren sich von Periode zu Periode die $\mathrm{z}$ insen erhöhen, attestieren die Monetaristen diesen BestandsgröBenimpulsen einen dämpfenden EinfluB auf die private Ausgabenneigung und belegen unter anderem auf diese Weise ihre These vom mittelfristigen crowding-out (subsequent-round crowding-out), das heiBt einer Verdrăngung von Privatausgaben in den anfänglichen Perioden der reinen Finanzierungsphase des Defizits ${ }^{2}$.

1) Vgl. zu dem Begriffspaar first-round crowding-out und subsequent-round crowding-out: Floyd, J.E.. Heynes, J.A., Deficit Finance and "First-Round" Crowding Out: A Clarification, in: Canadian Journal of Economics, 1978, S. 97 f.; Bruce, N., Purvis, D.D., Deficit Finance and "First-Round" Crowding Out: A Correction and Further Clarification, in: Canadian Journal of Economics, 1979, S. 728 ff.; Woglom, G. , First-Round "Crowding Out" in a Generalized IS-LM Model, in: Journal of Macroeconomics, 1979, S. 119.

2) Vgl. Friedman, M. Comments 
Langfristiges crowding-out bezieht sich auf das Ende der reinen Finanzierungsphase des Defizits. Dieses ist dann gekommen, wenn der öffentliche Haushalt wieder ausgeglichen ist, und sich deshalb die von den privaten Wirtschaftssubjekten gehaltenen Bestände an Staatspapieren nicht mehr erhöhen. Haben die Ausgaben. der Privaten $z u$ diesem zeitpunkt einen geringeren wert als die Referenzausgaben in der Entstehungsphase des Defizits, dann ist ein langfristiges crowding-out bewirkt worden ${ }^{1)}$ ?

In der folgenden tbersicht 1 werden noch einmal die verschiedenen Zeitpunkte und Zeiträume, zu und in denen ein crowding-out eintreten kann, angefuhrt und miteinander in Beziehung gebracht worden.

3. Finanzielles und güterwirtschaftliches crowding-out

Bisweilen wird zwischen finanziellem crowding-out und güterwirtschaftlichem crowaing-out unterschieden 2)

Entfaltet der staat auf dem Gütermarkt zusätzliche Nachfrage, so gelingt es ihm bei nicht ausdehnungsfähiger Produktion nur dann, weitere Güter an sich zu ziehen, soferr andere - die Privaten - zurückstehen. Setzt er seine Pläne durch, 1st das AusmaB der Verdrängung pri-

1) Vgl. z.B. Blinder, A.S., Solow, R.M., Does Fiscal Policy Matter?, in: Journal of Public Economics, 1973, S. $324 \mathrm{ff}$.

2) Vgl. Friedman, B., Crowding Out or Crowding In? Economic Consequences of Financing Government Deficits, a.a.0., S. $596 \mathrm{f}$.; Taylor, C.T., Crowding Out: Its Meaning and Significance, in: Cook, S.T., Jackson, P.M. (Hrsg.), Current Issues in Fiscal Policy, Oxford, 1979, S. 86; Wilson, T., Crowding Out: The Real Issues, in: Banca Nazionale Lavoro Quarterly Review, September 1979, S. $227 \mathrm{ff.}$; Verde, A., Crowding out Once More: An Attempt to Estimate Its Size in Italy, 1974-1977, in: Review of the Economic Conditions in Italy, 1979, S. $309 \mathrm{ff.;}$ Kitching, B., Real "Crowding-Out" as a Factor in the American Inflation-Recession, in: Zeitschrift für Nationalökonomie, 1972 , S. 289 ff. 
Ubersicht 1: Kurzfristiges, mittelfristiges und langfristiges crowding-out

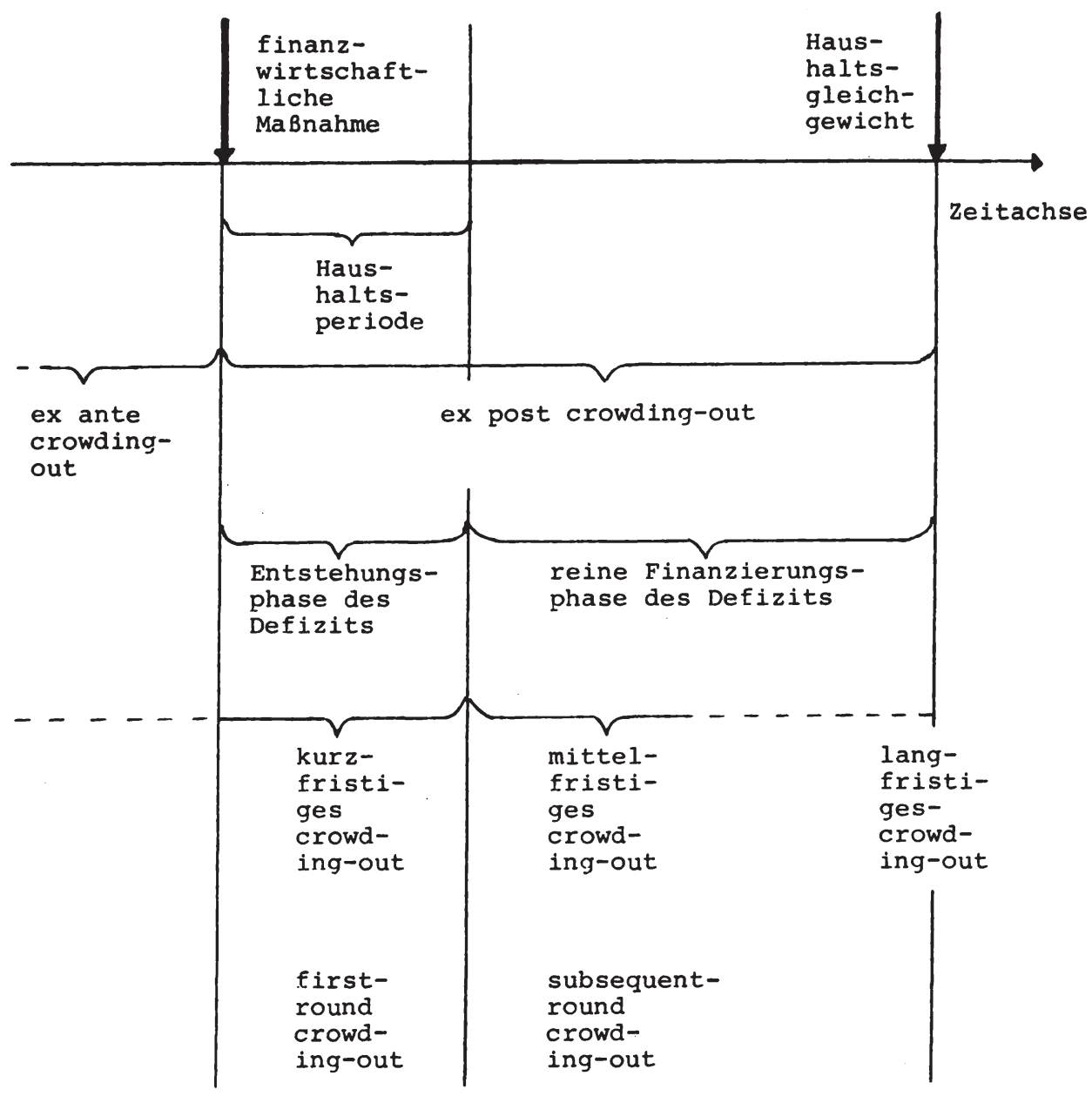

vater Nachfrage völlig unabhängig von der Art der Finanzierung der Zusatznachfrage, sondern wird nur von deren Umfang bestimmt. Dies ist das güterwirtschaftliche crowding- 
out; es ist zwangsläufige Folge einer starren Güterangebotsfunktion.

Beim finanziellen crowding-out wird dagegen explizit auf die Finanzierung zusätzlicher Transformationsausgaben und die damit verbundenen Anpassungsreaktionen auf den Finanzmärkten abgestellt; es werden also die Transmissionsketten in den Vordergrund gerückt, welche uber die Finanzmärkte laufen. Der Annahme einer nicht expansionsfähigen Produktion kommt keine Bedeutung $z u$. Finanzielles crowding-out kann auch bel elner preiselastischen Guterangebotsfunktion stattfinden.

D. Effekt

1. Partielles, vollständiges und ubermäßiges crowding-out

Im Hinblick auf das AusmaB der Verdrängung kann zwischen partiellem, vollständigem und übermäBigem crowding-out unterschieden werden ${ }^{1}$ !

Bleibt nach einer Erhöhung der staatlichen Güternachfrage die Gesamtnachfrage konstant, so ist die private Güternachfrage in genau dem gleichen Umfang zurückgedrängt worden; es liegt eln vollständiges crowding-out vor. Partiell ist das crowding-out dann, wenn die privaten Ausgaben zwar sinken, aber um weniger, als die staatliche Güternachfrage steigt, so daß die Gesamtausgaben zunehmen. Vermindert sich dagegen die Güternachfrage der Privaten um mehr, als sich die des Staates erhoht, so ist ein ubermäBiges crowding-out bewirkt worden; die Gesamtausgaben nehmen ab.

Die Formen von crowding-out nach MaBgabe des AusmaBes der Verdrängung werden in Ubersicht 2 noch einmal mit Hilfe des Gesamtausgabenmultiplikators [dY/dG] und des Privatausgabenmultiplikators $[\mathrm{d}(\mathrm{C}+\mathrm{I}) / \mathrm{dG}]$ verdeutlicht.

1) Vgl. Carlson, M.K., Spencer, R.W., Crowding-Out and its Critics, a.a.0., s. 3 
Ubersicht 2: Partielles, vollständiges und übermäBiges crowding-out

\begin{tabular}{l|c|c}
$\begin{array}{l}\text { Ausma B des } \\
\text { crowding-out }\end{array}$ & $\begin{array}{l}\text { Wert des Gesamt- } \\
\text { ausgabenmultipli- } \\
\text { kators }\end{array}$ & $\begin{array}{l}\text { Wert des Pri- } \\
\text { vatausgaben- } \\
\text { multiplikators }\end{array}$ \\
\hline vollständig & $\frac{d Y}{d G}=0$ & $\frac{d(C+I)}{d G}=-1$ \\
\hline partiell & $0<\frac{d Y}{d G}<1$ & $-1<\frac{d(C+I)}{d G}<0$ \\
\hline übermäBig & $\frac{d Y}{d G}<0$ & $\frac{d(C+I)}{d G}<-1$
\end{tabular}

2. Reales und nominelles crowding-out

In Abhänglgkeit davon, ob man bei der Verdrängung auf die realen oder auf die nominellen Privatausgaben abstellt, wird zwischen realem und nominellem crowdingout differenziert ${ }^{1}$ !

Bleibt das Preisniveau im Zuge finanzwirtschaftlicher Aktivitäten konstant, kommt es stets auch zu einem nominellen crowding-out, wenn die realen Ausgaben der Privaten sinken. Bei einem variablen Preisniveau ist es indessen möglich, daB sich die nominellen Privatausgaben in eine andere Richtung entwickeln als die realen Privatausgaben. Diese Konstellation tritt dann ein, wenn das preisniveau infolge einer expansiven finanzwirtschaftlichen MaBnahme steigt und die

1) Vgl. Carlson, M.K., Spencer, R.W., Crowding-Out and its Critics, a.a.0., S. 16 f. sowie Argy, V., Fiscal Policy and Crowding Out in an Open Economy, unveröffentlichtes Diskussionspapier, 1977, S. 2. 
nominellen Ausgaben der Privaten zunehmen, aber mit einer geringeren Rate als das Preisniveau. In nomineller Betrachtung wird dann ein pulling-in, in realer ein crowding-out bewirkt.

Viertes Kapitel: Zum Untersuchungsrahmen

A. Impuls

1. Die Finanzierungsrestriktion des Staates

Für die finanziellen Transaktionen jeder Wirtschaftseinheit - und damit auch für den staat ${ }^{1)}$ - gilt, daB sich innerhalb einer Periode MittelzufluB und MittelabfluB stets die Waage halten, sich also Einnahmen und zunahme der Verbindlichkeiten auf der einen und Ausgaben und $\mathrm{zu}$ nahme der Forderungen auf der anderen seite in summa entsprechen. Das heiBt für den staat nichts anderes, als daB er bel seinen finanzwirtschaftlichen MaBnahmen innerhalb einer Haushaltsperiode einer Finanzierungsrestriktion unterliegt; die Staatsausgaben sind mit Einnahmen sowie einer (Netto-) Kreditaufnahme zu finanzieren ${ }^{2}$.

1) Wir halten den "Staat" und den "Staatssektor" begrifflich wie folgt auseinander: der "Staat" ist gleich den Gebietskörperschaften, der "Staatssektor" umfaBt die Gebietskörperschaften und die Notenbank.

2) Die Finanzierungsrestriktion des Staates 1st von Ott, D.J., Ott, A.F., Budget Balance and Equilibrium Income, Journal of Finance, $1965, \mathrm{~S} .71 \mathrm{ff}$. sowie von Christ, C.F., A Simple Macroeconomic Model with a Government Budget Restraint, in: Journal of Political Economy, 1968, S. $53 \mathrm{ff}$. in die crowding-out-Debatte elngefuhrt worden; ihr kommt insbesondere im Zusammenhang mit Vermögenseffekten und einem mittel- und langfristigen crowding-out eine hervorragende Bedeutung $\mathrm{zu}$; vgl. hierzu: Burrows, P., The Government Constraint and the Monetarist-Keynesian-Debate, in: Cook, S.T., Jackson, P.M. (Hrsg.), Current Issues in Fiscal Policy, Oxford, 1979, S. $69 \mathrm{ff.;} \mathrm{für} \mathrm{eine} \mathrm{Einordnung} \mathrm{der}$ staatlichen Finanzierungsrestriktion in die Theorie des Geldangebots vgl. Emrich, R., Die Theorie des Geldangebots in einer geschlossenen und in einer offenen Volkswirtschaft, Mainzer Dissertation, 1977, S. $32 \mathrm{ff}$. 
Die Ausgaben des Staates unterscheiden wir nach dem auf Pigou und Colm zurückgehenden Kriterium der Wirkung auf die gesamtwirtschaftliche Güternachfrage in

- Transformationsausgaben (G), d.h. Zahlungen an private Haushalte und Unternehmen für Güter und Dienste und

- Transferzahlungen (TR), d.h. Zahlungen an private Haushalte und Unternehmen ohne Gegenleistung.

Bei der Finanzierung seiner Ausgaben kann der Staat, so nehmen wir an, unter drei Möglichkeiten auswählen:

- Steuereinnahmen $\left(T^{b}\right)$,

- Kreditaufnahme bei der Notenbank (Notenbankkredit), d.h. Erhöhung des Bestandes an Staatsschuldtiteln bei der Notenbank $\left(z-z_{-1}\right)$,

- Kreditaufnahme bei den privaten Haushalten und Unternehmen (Kapitalmarktkredit), d.h. Erhöhung des Bestandes an Staatsschuldtiteln bei den Privaten $\left(B-B_{-1}\right)$.

Die Kreditaufnahme des Staates bei der Notenbank und bei den Privaten innerhalb einer Haushaltsperiode ist der staatliche Finanzierungssaldo (D):

$$
(1-4-1) \quad D=\left(\mathrm{Z}-\mathrm{Z}_{-1}\right)+\left(\mathrm{B}-\mathrm{B}_{-1}\right)
$$

Als Finanzierungsrestriktion des staates ergibt sich:

$$
(1-4-2) \quad G+T R=T^{b}+\left(Z-Z_{-1}\right)+\left(B-B_{-1}\right)
$$

Wenn Transfers und Bruttosteuereinnahmen zu Nettosteuereinnahmen (T) zusammengefaBt werden, folgt ${ }^{1}$ )

1) Bent Hansen unterscheidet zwei Typen von staatlichen Finanzierungsrestriktionen, welche die unterschiedlichen institutionellen Bedingungen der Geld- und Finanzpolitik in Europa und der USA zum Ausdruck bringen sollen: "US-Typ" und "Europäischer Typ". Im Gegensatz zum "Europăischen Typ" fehlt im "US-Typ" die Möglichkeit der Notenbankfinanzierung. $\mathrm{Da}$ in der Bundesrepublik Deutschland die Notenbank dem Staat - wie in den USA - nicht als Hausbank zur Verfügung steht, spiegelt der "US-TYp" die deutschen Verhältnisse eher wider. Vgl. Hansen, B., On the Effects of Fiscal and Monetary Policy: A Taxonomic Discussion, in: American Economic Review, 1973, S. $549 \mathrm{ff.;}$ vgl. auch Dieckheuer, G., Eine dynamische Ana-

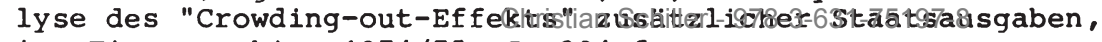

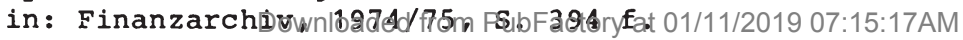


$(1-4-3) \quad G=T+\left(Z-Z_{-1}\right)+\left(B-B_{-1}\right)$

oder in Anderungsgrößen geschrieben ${ }^{1)}$ :

$(1-4-4) d G=d T+d z+d B$.

Indem der staat die finanziellen Mittel, die er sich bei der Notenbank beschafft, verausgabt, erhöht sich, sofern die Notenbank nicht im Gegenzug kompensierende Maßnahmen ergreift, das von den Privaten gehaltene Zentralbankgeld $\left(\mathrm{MZ}-\mathrm{MZ}_{-1}\right)$ :

$(1-4-5) \quad Z-Z_{-1}=M Z-M Z_{-1}$.

Wird Gleichung $(1-4-5)$ in die Finanzierungsrestriktion (1-4-3) eingesetzt und wird umgruppiert, folgt

$(1-4-6) \quad G-T=(M Z-M Z-1)+\left(B-B_{-1}\right)$

und wieder in Anderungsgrößen geschrieben:

$(1-4-7) \quad d G-d T=d M Z-d B$.

Monetaristen unterteilen eine finanzwirtschaftliche MaBnahme gedanklich in rein fiskalische Impulse und in finanzielle Impulse. Die rein fiskalischen Impulse sind die Verănderung von Ausgaben und Steuern, die finanziellen Impulse das Arrangement zur Deckung eines Finanzierungssaldos. In der staatlichen Finanzierungsrestriktion (1-4-7) kommen die rein fiskalischen Impulse mithin auf der linken, die finanziellen Impulse auf der rechten

1) Die Bestandsänderung $\mathrm{dz}_{-1}$ und $\mathrm{dB}$ entsprechen in ihrer Summe dem Finanziērungssald̄ol der Vorperiode und sind für die laufende Periode Daten. Bei einem ausgeglichenen Haushalt in der Vorperiode nehmen sie den Wert Null an. Von dieser Annahme gehen wir durchweg aus. 
Seite zum Ausdruck ${ }^{1)}$ :

$(1-4-7)$



rein fiskalische Impulse
$=$

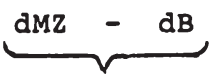

finanzielle Impulse.

\section{Wirkungskonzept: Budgetinzidenz}

Je nachdem, welche Politikvariablen in elner Wirkungsanalyse verändert werden, hat man es bei den Effekten mit spezifischen Wirkungen, Budgetwirkungen oder Differentialwirkungen zu $\tan ^{2 !}$ ?

Das Konzept der spezifischen Inzidenz findet häufig in Lehrbüchern Anwendung, wenn die Wirkung kreditmarktfinanzierter Transformationsausgaben anhand des "Keynesianischen Kreuzes" plausibel gemacht wird; lediglich der Ausgabenimpuls wird dabei betrachtet ${ }^{3}$. Diese Vorgehensweise ist jedoch nicht kompatibel mit der faktisch vorhandenen Beschränkung der Handlungsfreiheit finanzpolitischer Entscheidungsträger, welche ihnen durch die Finanzierungsrestriktion auferlegt ist $^{4)}$ : Bei n finanzwirtschaftlichen parametern können nur n-1 Parameter unabhängig voneinander variiert werden. Verändert der staat einen finanzwirtschaftlichen Parameter, z.B. die Transformationsausgaben, muB uno actu zumindest noch eine weitere Variable der staatlichen Finanzierungsrestriktion angepaBt werden ${ }^{5}$ ! Um ein vollständiges Bild des

1) Vgl. Brunner, K., The "Monetarist Revolution" in Monetary Theory, in: Weltwirtschaftliches Archiv, 1970, S. 21 sowie Neumann, M.J.M., Schröder, W., Aspekte monetaristischer Theorie, in: Konjunkturpolitik, 1975, S. $40 \mathrm{ff}$.

2) Die Terminologie lehnt sich an Musgrave, R., Finanztheorie, Tübingen, $1974^{2}$, S. $158 \mathrm{ff}$. an.

3) Vgl. z.B. Baird, C.W., Macroeconomics. An Integration of Monetary, Search, and Income Theories, Chicago u.a., 1973, S. $38 \mathrm{ff}$.

4) Vgl. Cavaco-Silva, A.A., Economic Effects of Public Debt, London, 1977, S. $2 \mathrm{ff.}$

5) Vgl. Christ, C.F., A Simple Macroeconomic Model with a Government Budget Restraint, a.a.O., S. 55 sowie Siebke, J., Die Berücksichtigung der Budgetbeschränkung des Staates in dem Keynesianischen Makrosystem.

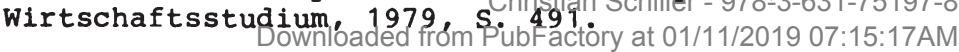


Effekts der finanzwirtschaftlichen Aktivität zu erhalten, müssen mithin zu den spezifischen Wirkungen des Ausgabenimpulses zwingend die des Finanzierungsimpulses hinzugefügt werden ${ }^{1}$ !

Bei Differentialwirkungen und Budgetwirkungen wird der korrespondierende Primärimpuls mit in die Analyse einbezogen. In crowding-out-Analysen kommt vorwiegend das Konzept der Budgetwirkungen zur Anwendung; dieser Vorgehensweise schlieBen wir uns an. Nicht in unserer Analyse betrachtet werden folglich alle finanzwirtschaftlichen Stabilisierungsmaßnahmen, im Zuge derer das Niveau der Staatsausgaben konstant bleibt. Dies sind z.B.: eine SteuerermäBigung und entsprechende Erhöhung des Finanzierungsdefizits (deficit without spending), eine Variation der struktur einer gegebenen staatsschuld (debt management) sowie eine gegenläufige Veränderung von Transformationsausgaben und Transferzahlungen.

3. Ausgabenart und Finanzierungsart:

Transformationsausgaben und Kapitalmarktfinanzierung

Für die finanzwirtschaftlichen Primärimpulse, die eine Veränderung privater Ausgaben zum Ergebnis haben, steht, wie gesagt, in unserer Analyse eine expansive MaBnahme des Staates, durch die das Budget verlängert wird.

Von den Ausgabealternativen betrachten wir ausschlieBlich die Transformationsausgaben ${ }^{2}$ ! Gerade diese werden von Keynesianern als besonders wirksam eingeschätzt, wenn es

1) DaB Finanzierungsimpulse ignoriert werden, wurde bisweilen damit gerechtfertigt, sie seien vernachlässigbar gering. Diese Einschätzung herrschte lange zeit vor, hat jedoch mit der Renaissance der crowding-out-These erheblich an Boden verloren.

2) Dies ist auch die in der Literatur übliche Vorgehensweise. Eine Ausnahme bilden z.B. Abrahams, B.A., Schitz, M.D., The "Crowding-Out" of Government Transfers on Private Charitable Contributions, in: Public Choice, 1978 , S. 29 ff. 
um die Anhebung des Gesamtausgabenniveaus geht ${ }^{1}$ ! Grund dafür ist, daB Transformationsausgaben im Gegensatz zu Transferzahlungen Bestandteil der gesamtwirtschaftlichen Güternachfrage sind. Diese nimmt mithin uno actu $z u$, wenn der Staat mehr Transformationsausgaben tätigt. Durch zusätzliche Transferzahlungen steigt das Gesamtausgabenniveau dagegen nur mittelbar und zwar insoweit, wie dadurch ihre Empfänger zur Tätigung von Konsum- und Investitionsgüterkäufen veranlaBt werden.

Je nachdem, welche Finanzierungskomponente komplementär zur Transformationsausgabenerhöhung angepaBt wird, können anhand der staatlichen Finanzierungsrestriktion (1-4-2) die drei in tbersicht 3 aufgeführten Politikvarianten unterschieden werden ${ }^{2}$ !

Utbersicht 3: Politikvarianten

\begin{tabular}{l|l|l}
$\begin{array}{l}\text { Veränderte } \\
\text { Politik- } \\
\text { variablen }\end{array}$ & $\begin{array}{l}\text { Konstante } \\
\text { Politik- } \\
\text { variablen }\end{array}$ & Bezeichnung \\
\hline $\mathrm{dG}=\mathrm{dT} \mathrm{T}^{\mathrm{b}}$ & $\mathrm{dTR}=\mathrm{dB}=\mathrm{dMZ}=0$ & $\begin{array}{l}\text { "pure fiscal } \\
\text { policy" }\end{array}$ \\
\hline $\mathrm{dG}=\mathrm{dMZ}$ & $\mathrm{dTR}=\mathrm{dB}=\mathrm{dT}=0$ & $\begin{array}{c}\text { "monetary- } \\
\text { fiscal policy" }\end{array}$ \\
\hline $\mathrm{dG}=\mathrm{dB}$ & $\mathrm{dTR}=\mathrm{dT}=\mathrm{dMZ}=0$ & $\begin{array}{c}\text { "bond-financed } \\
\text { fiscal policy" }\end{array}$
\end{tabular}

1) Vgl. z.B. Schäfer, C., Zur Notwendigkeit und Legitimation einer mittelfristigen beschäftigungssichernden Finanzpolitik, in: WSI-Mitteilungen, $1980, \mathrm{~S} .635 \mathrm{f}$.

2) Vgl. Burrows, P., The Government Budget Contraint and the Monetarist-Keynesian Debate, a.a.0., S. 70; vgl. auch Christ, C.F., A Simple Macroeconomic Model with a Government Budget Restraint, a.a.0., S. 57 f. 
Der aggregative Effekt einer "pure fiscal policy" wird wenig kontrovers beurtellt; allgemein akzeptiert man ein crowding-out. Lediglich in der Diskussion um das Haavelmo-Theorem wurde die Ansicht vertreten, der "balanced-budget-multiplier" habe einen Wert von Eins, d.h. die Privatausgaben blieben infolge elner steuerfinanzierten Transformationsausgabenerhøhung konstant. $\mathrm{DaB}$ die steuererhohung, fur sich genommen, die private Güternachfrage zurückdrängt, wurde auch von den Vertretern des Haavelmo-Theorems nicht bestritten. Die kontraktive Primärwirkung der steuererhöhung veranschlagten sie jedoch nur auf $c \cdot \mathrm{dT}^{\mathrm{b}}(\mathrm{c}=$ marginale Konsumquote). Da dieser eine expansive Primärwirkung der Transformationsausgabenzunahme in Höhe von dG gegenübersteht, ergibt sich per saldo insgesamt ein expansiver AnstoBimpuls in Höhe von $(1-c)$.dG und bei einkommensabhängigen privaten Konsumausgaben ein Gesamtausgabeneffekt von:

$$
(1-4-8) \quad d Y=\frac{1}{1-c}(1-c) \cdot d G=d G
$$

Die Privatausgaben bleiben unverändert, denn es gilt:

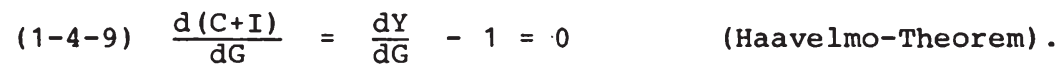

Unter den Prämissen des Haavelmo-Theorems regen zusätzliche Transformationsausgaben genau so viele private Ausgaben an, wie durch die steuererhöhung verdrängt werden. Da die Prämissen äußerst restriktiv sind" ${ }^{1}$, wird jedoch allgemein von einem gröBer werdenden, ausgeglichenen Budget kein wesentlicher aggregativer Effekt erwartet.

Zur Steuerfinanzierung konträr, aber ebensowenig kontrovers, wird die Notenbankfinanzierung von zusätzlichen Transformationsausgaben eingeschätzt. In diesem Fall beschafft sich der staat die erforderlichen finanziellen

1) James Buchanan listet sieben Voraussetzungen auf:, vgl. Buchanan, J., The Public Finances, Homewood, Ill., 1965, S. $78 \mathrm{ff}$. 
Mittel bei der Notenbank, und es treten im privaten Sektor keine monetären Entzugseffekte auf; vielmehr wird, wenn die Notenbank nicht dagegenhäl, durch die Verausgabung der Mittel die Zentralbankgeldmenge vergrößert.

Umstritten zwischen Monetaristen und Keynesianern 1st allerdings, ob der (unbestrittene) aggregative Effekt solch einer "monetary-fiscal pollcy" mehr der "fiscal policy" oder der "monetary policy" zuzurechnen 1st". Keynesianer halten zum einen die Transformationsausgabenerhohung - in der Terminologie der Monetaristen: den rein fiskalischen Impuls für in aggregativer Hinsicht bedeutsam und rechnen zum anderen unter Berufung auf das Veranlassungsprinzip sämtliche Effekte, die durch die Erhöhung der Zentralbankgeldmenge bewirkt werden, ebenfalls der Finanzpolitik zu. Demgegenüber hängt nach Ansicht der Monetaristen die Gesamtwirkung vor allem von der Änderung des Bestandes an Zentralbankgeld bei den Privaten $a b$, und diese wird von ihnen nicht unter die Finanz- sondern die Geldpolitik subsumiert ${ }^{2}$ !

Im Kern der crowding-out-Debatte geht es um den aggregativen Effekt einer zusätzlichen Güternachfrage des Staates, welche durch eine Kreditaufnahme bei den Privaten finanziert wird.

1) "Sure enough, there is no controversy over Government spending financed by printing money. Both sides agree that it will be expansionary. The only difference is that one group likes to call it fiscal policy, while the other prefers to call it monetary policy". Arestis, P., The "Crowding-Out" of Private Expenditures by Fiscal Actions: An Empirical Investigation, in: Public Finance, 1979 , S. 46 .

2) Vgl. hierzu Keran, M.W., Monetary and Fiscal Influence on Economic Activity. The Historical Evidence, a.a.0., s. 23 ff.; Fand, D., Some Issues in Monetary Economics, in: Federal Reserve Bank of St. Louis Review, Januar 1970, S. $23 \mathrm{ff}$. sowie die Diskussion zwischen Neldner und Neuman/Schröder: Neldner, M., Einige problematische Aspekte der monetaristischen Stabilisierungspolitik, in: Konjunkturpolitik, 1975, S. $24 \mathrm{ff.;} \mathrm{Neumann,} \mathrm{M.J.M.,} \mathrm{Schröder,} \mathrm{W.,} \mathrm{Aspekte} \mathrm{moneta-}$ ristischer Theorie, a.a.O., S. $39 \mathrm{ff.;}$ Neldner, M., Aspekte monetaristischer Theorie: Eine Erwiderung, in: Konjunkturpolitik, 1979, S. $52 \mathrm{f}$. 
Zum einen ist der Dissens hier am gröBten: Keynesianer erwarten z.B. von einer Kreditmarktfinanzierung bei Unterbeschäftigung nur unbedeutende monetäre Entzugseffekte; Monetaristen widersprechen dem und verweisen insbesondere auf sich kumulierende und die Ausgabenneigung der Privaten dämpfende BestandsgröBenimpulse im Zuge der Emission von Staatsschuldtiteln. Zum anderen ist diese Variante die für die finanzpolitische Praxis wichtigste der genannten Politikvarianten. Denn erstens werden durch die Kreditmarktfinanzierung die Kontraktionswirkungen einer Steuererhöhung vermieden und zweitens ist der Weg der Mittelbeschaffung bei der Notenbank häufig, wie auch in der Bundesrepublik Deutschland, dem Staat weitgehend versperrt und auch unerwünscht.

Die crowding-out-Debatte ist deswegen hauptsächlich eine Kontroverse um die Eignung von kreditmarktfinanzierten zusätzlichen Transformationsausgaben als Mittel der Stabilisierungspolitik. Wir beschränken uns in unserer Wirkungsanalyse auf diese Politikvariante ${ }^{1 !}$

4. Zur Abgrenzung finanzwirtschaftlicher Impulse von geldpolitischen Impulsen

Finanzwirtschaftliche Impulse nehmen bei ihrer Transmission auf die Güternachfrage der Privaten auch den Weg über die monetären Märkte und beeinflussen zunächst einmal monetäre Variablen, wie Geldbestände und Zinsen, ehe sie sich auf den Gütermarkt fortpflanzen. In diesem Fall sind die Transmissionsvariablen finanzwirtschaftlicher Primärimpulse die-

1) Bei den hier angesprochenen Varianten handelt es sich um "reine" Politikvarianten, welche dadurch gekennzeichnet sind, daB jeweils alle auBer zwei Politikvariablen der staatlichen Finanzierungsrestriktion konstant bleiben. Wo in unserer Wirkungsanalyse ein einkommensabhängiges Steueraufkommen berücksichtigt wird, varileren jedoch bei einer kreditär finanzierten Vermehrung der Transformationsausgaben in der Regel drei Politikvariablen: die Transformationsausgaben werden autonom erhöht, das steueraufkommen verändert sich nach Maßgabe der Einkommensentwicklung und die Finanzierungslücke wird durch eine Kreditaufnahme bei den Privaten geschlossen. 
selben, wie diejenigen, welche der tbertragung geldpolitischer Impulse in den privaten Aktivitätsbereich dienen. Finanzwirtschaftliche Maßnahmen lösen mithin im monetären Bereich Effekte aus, die ebenso durch geldpolitische MaBnahmen induziert und damit auch kompensiert werden können. Die Frage, wie monetäre Teilimpulse finanzwirtschaftlicher MaBnahmen in Wirkungsanalysen zu behandeln sind, welche davon nicht der Finanzpolitik, sondern der Geldpolitik zugerechnet werden sollen, wird unterschiedlich beantwortet ${ }^{1}$ !

Bent Hansen ${ }^{2)}$ schlägt z.B. vor, die Grenzlinie des Wirkungsbereichs der Finanzpolitik anhand der Relation der Bestände an Zentralbankgeld und an Staatsschuldtiteln in Händen der Privaten zu ziehen (vgl. Ubersicht 4). Mit den Monetaristen unterteilt er eine finanzwirtschaftliche MaBnahme in eine rein fiskalische und in eine finanzielle Komponente. Die finanzielle Komponente stellt sich aus Sicht der Privaten als eine Zunahme ihrer Forderungen gegenüber der Notenbank und gegenüber dem Staat dar (Notenbank + Staat = Staatssektor). Diese zerlegt Hansen nochmals: zum einen in die

1) Einen Uberblick zum Problem der Abgrenzung von Geld- und Finanzpolitik vermitteln Forte, F., Hochmann, H.M., Monetary and Fiscal Policy: Ambiguities in Definitions, in: Haller, H., Recktenwald, H.C. (Hrsg.), Finanz- und Geldpolitik im Umbruch, Festschrift für R. Nöll von der Nahmer, Mainz, 1969 , S. $357 \mathrm{ff.}$ und Machlup, F., The Effects of Fiscal Policy and the Choice of Definitions, in: Greenfield, H.I. u.a. (Hrsg.), Theory for Economic Efficiency: Essays in Honor of Abba P. Lerner, Cambridge, Mass., London, 1979, S. 92 ff.; vgl. auch Lachmann, W., Crowdingout und die Frage nach der neutralen Geldpolitik, in: Kredit und Kapital, 1978, s. 451 ff.; Pohl, Reinhard, Einige geldtheoretische und geldpolitische Probleme des crowding-out des privaten sektors durch den staat, in: Ehrlicher, W. (Hrsg.), Geldpolitik, Zins und Staatsverschuldung, Schriften des Vereins für Socialpolitik, Band 111,1981 , S. 265 ff.

2) Vgl. Hansen, B., The Economic Theory of Fiscal Policy, London, 1958, S. $30 \mathrm{ff.;}$ vgl. auch Lachmann, w., Crowdingout und die Frage nach der neutralen Geldpolitik, a.a.o., S. $459 \mathrm{ff}$. 
Impulse einer Veränderung des Niveaus der Forderungen gegenüber dem Staatssektor [finanzielle Niveauimpulse = $\mathrm{d}(\mathrm{MZ}+\mathrm{B})]$ und zum anderen in die Impulse der verănderung der Struktur der Forderungen gegenüber dem staatssektor [finanzielle strukturimpulse $=d(\mathrm{MZ} / \mathrm{B})]$. Die finanzielien

Ubersicht 4: Abgrenzung von Geld- und Finanzpolitik nach Bent Hansen

$(1-4-7) \mathrm{dG}-\mathrm{dT}=\mathrm{dMZ}+\mathrm{dB}$

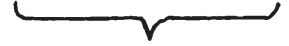

rein fiskalische Impulse

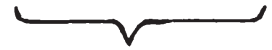

finanzielle Impulse
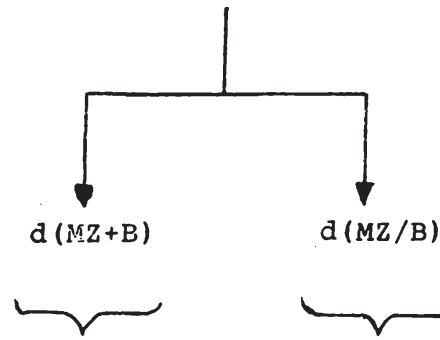

finanzielle Niveauimpulse finanzielle

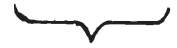

Strukturimpulse

Finanzpolitik

Geldpolitik 
Strukturimpulse ordnet Hansen dem Wirkungsbereich der Geldpolitik zu.

Verändert sich im zuge einer kreditmarktfinanzierten Vermehrung der Transformationsausgaben die Relation zwischen den von den Privaten gehaltenen unverzinslichen und verzinslichen Forderungen gegenuber dem Staatssektor, dann ist dies nach der Abgrenzung von Hansen ein geldpolitischer Impuls, und damit bewirkte Effekte im privaten Aktivitätsbereich sind nicht der Finanzpolitik zuzurechnen. Ergebnis: Nach Hansen düren in Wirkungsanalysen, in denen allein die Effekte finanzwirtschaftlicher Maßnahmen untersucht werden und in denen sich die Notenbank neutral verhält, finanzielle strukturimpulse nicht auftreten $[d(M Z / B)=0]$. Es muB dort folglich die Notenbank dafür sorgen, daB nach den finanzwirtschaftlichen Impulsen die Forderungen gegenüber dem staatssektor keine andere struktur als vorher aufweisen. Dies erfordert mithin kompensierende offenmarkttransaktionen, wenn der Staat zur Ausgabenfinanzierung Schuldtitel an die privaten verkauft.

Wir folgen nicht dem Vorschlag von Hansen, sondern identifizieren mit Currie ${ }^{1)}$ geldpolitische Impulse anhand einer Zwischenzielvariablen der Geldpolitik. Dies sind Größen des monetären Bereichs, die den Trägern der Geldpolitik als steuerungsgröBe dienen, um letztlich relevante wirtschaftspolitische zielsetzungen (Endziele) zu erreichen. Auf geldpolitische $\mathrm{Zwischenzielvariablen} \mathrm{wirken}$ neben geldpolitischen Impulsen auch (für die Geldpolitik) exogene Impulse, z.B. monetäre Teil.impulse finanzwirtschaftlicher MaBnahmen, ein. Aus Sicht der Geldpolitik exogene Impulse werden von den geldpolitischen Trägern im Hinblick auf ihren Zwischenzieleffekt überprüft und

1) Vgl. Currie, D.A., Monetary and Fiscal Policy and the Crowding-Out Issue, unveröffentlichtes Diskussionspapier, Mai 1978 , S. $4 \mathrm{f}$. 
sodann gegebenenfalls durch gej.dpolitische MaBnahmen kompensiert. Ein Nichtauftreten von geldpolitischen Impulsen heiBt nach diesem Separationskonzept, daB die von der Notenbank verwendete Zwischenzielvariable auf ihrem angestrebten Niveau verbleibt.

Ob sich als Zwischenzielvariable eher der Zins oder die Geldmenge eignet, ist zwischen Monetaristen und Keynesianern umstritten 1) Folgen wir dem keynesianischen Vorschlag der Strategie der zinsfixierung und trennen wir z.B. mit Meade $^{2)}$ geldpolitisch induzierte monetäre Impulse von finanzwirtschaftlich bewirkten monetären Impulsen anhand des zinsniveaus, hat dies für unsere Wirkungsanalyse folgende Konsequenz: Eine allfällig zu beobachtende Veränderung des Zinsniveaus ist ein geldpolitischer Impuls. Damit von der Geldpolitik keine Impulse ausgelöst werden, muB das zinsniveau jedoch konstant bleiben. Dies macht ein permissives Verhalten der Notenbank im Hinblick auf die Geldmenge erforderlich. Deshalb können finanzwirtschaftliche Primärimpulse auch über Geldmengeneffekte in den privaten Aktivitätsbereich übertragen werden. Zinseffekte entfallen indessen als Transmissionsriemen eines crowding-out.

Monetaristen bevorzugen Geldbestände als geldpolitische Zwischenzielvariablen. Trennen wir die Impulse der Geldpolitik von monetären Teilimpulsen einer finanzwirtschaftlichen Maßnahme anhand der Geldmenge, erfordert eine $\mathrm{Ab}-$

1) Vgl. Mayer, T., Die Struktur des Monetarismus, in: Ehrlicher, W., Becker, W.-D. (Hrsg.), Die Monetarismus Kontroverse, Beihefte zu Kredit und Kapital, Nr. 4, 1978, S. 38 ff.

2) Nach Meade erfordert eine Abwesenheit geldpolitischer Impulse "that the banking system must be prepared to expand (or contract) the total supply of money to the extent necessary to prevent any scarcity (or plenty) of funds in the capital market which may be induced by any other disturbing factor, from causing a rise (or fall) of interest rates"., Meade, J.E., The Balance of Payments, London, New York, 1952, S. 48; vgl. auch Machlup, F., Fiscal folicy and the Choice of Definitions, a.a.o., s. $95 \mathrm{ff}$. 
wesenheit von geldpolitischen Impulsen Konstanz der Geldmenge ") Die Geldmenge andert sich mithin nicht, wenn der Staat zusätzliche Ausgaben tätigt und diese kreditär finanziert. Das Zinsniveau kann demgegenüber variieren; dies ist dann ein monetărer Impuls der finanzwirtschaftlichen MaBnahme.

Abgesehen von dem Kapitel, in dem es um Preiseffekte geht, unterstellen wir nahezu durchweg eine konstante Geldmenge ${ }^{2)}$ und rechnen $z u$ beobachtende zinseffekte dem Wirkungsbereich der Finanzpolitik zu.

5. Zusammenfassung

Sämtliche staatliche Aktivitäten, wie z.B. auch ein "moral suasion", können Impulse für ein crowding-out sein. Im folgenden werden nur budgetwirksame betrachtet, im Zuge derer der staat ${ }^{3)}$ auf dem Gütermarkt mehr nachfragt und diese Zusatzausgaben durch Emission von Staatsschuldtiteln an die privaten Haushalte und Unternehmen finanziert. Zudem soll es sich um Impulse handeln, mit denen eine Erhöhung der Privatausgaben aus stabilisierungspolitischen Erwăgungen beabsichtigt

1) Vgl. Friedman, M., The Role of Monetary Policy, in: American Economic Review, 1968, S. 1 ff. Sowie Machlup, F., The Effects of Fiscal Policy and the Choice of Definitions, a.a.0., s. $99 \mathrm{f}$.

2) Konstanz der Geldmenge in einer stationären Wirtschaft entspricht in einer evolutorischen Wirtschaft eine jährliche Geldmengenzunahme nach Maßgabe des Wachstums des Produktionspotentials.

3) In einem weiteren Sinne können Verdrängungsimpulse überall und nicht nur im Staatssektor ausgelöst werden; auch der strukturwandel im privaten sektor ist eine Verdrängung, allerdings eine intrasektorale. Diesbezüglich ist die Begriffsfassung in der Literatur eindeutig: Crowding-out ist stets eine intersektorale Verdrängung mit dem staat als verdrängendem und den Privaten als Verdrängten. 
ist" Die Notenbank verhält sich dabei in dem Sinne neutral, daB (Preiseffekte und den Pesek/ Saving-Effekt ausgenommen) die Geldmenge konstant bleibt.

\section{B. Transmission}

1. Betrachtungszeitraum: kurze und mittlere Frist

Bezüglich der Transmission finanzwirtschaftlicher Impulse in den privaten Aktivitätsbereich bedarf es zweier Klärungen: Zum einen muB der Zeitraum fixiert wexden, auf den die Wirkungsanalyse ausgedehnt wird, und zum anderen muB die zu Beginn des Zeitraumes geltende gesamtwirtschaftliche Ausgangslage spezifiziert werden, auf welche die finanzwirtschaftlichen Impulse treffen.

Es gibt crowding-out-Analysen, in denen der Betrachtungszeitraum in der nahen und andere, in denen er in der fernen Zukunft endet ${ }^{2 !}$.

Im Rahmen langfristiger Analysen steht hauptsächlich das Problem im Mittelpunkt, ob gesamtwirtschaftliche Stabili-

1) Nur selten wird auch eine Verminderung privater Aktivitäten infolge staatlicher Interventionen, die nicht stabilisierungspolitisch, sondern allokations- oder distributionspolitisch intendiert sind, als crowding-out bezelchnet. Eine solche Ausnahme bilden z.B. Abrahams, B.A., Schitz, M.D. , The "Crowding-Out" Effect of Governmental Transfers on Private Charitable Contributions, a.a.0., S. $29 \mathrm{ff}$.

2) Vgl. Walther, H., రffentliche Verschuldung und "Crowdingout" Effekte, in: Nowotny, E. (Hrsg.), రffentliche Verschuldung, Stuttgart, New York, 1979, S. 65 ff.; Buiter, W.H., "Crowding out" and the Effectiveness of Fiscal Policy, a.a.O., S. 310 f.; Tobin, J., Deficit Spending and Crowding out in Shorter and Longer Runs, in: Greenfield, H.I. u.a. (Hrsg.), Theory for Economic Efficiency: Essays in Honor of Abba P. Lerner, Cambridge, Mass., London, 1979 , S. $217 \mathrm{ff}$. 
tät am Ende der reinen Finanzierungsphase mit einer Verdrängung privater Ausgaben vereinbar sein kann oder ob nicht vielmehr eine Ruhelage am Ende der reinen Finanzierungsphase einen positiven langfristigen Privatausgabenmultiplikator bedingt "

Bei kurz- und mittelfristigen crowding-out-Analysen geht es um das ursprüngliche Anllegen der Monetaristen, nămlich die Falsifizlerung der keynesianischen Hypothese von der aggregativen Effizienz eines finanzwirtschaftlichen demand management zur Beseitigung von Unterbeschäftigung. Genau dies ist unser Problem. Deshalb beschränken wir uns auf einen kurz- und mittelfristigen Betrachtungszeitraum. Die kurze Frist 1st die Entstehungsphase des Defizits, die mittlere sind die anfänglichen Perioden der reinen Finanzierungsphase.

\section{Gesamtwirtschaftliche Ausgangslage}

a) Unterbeschäftigung

Eine expansive Finanzpolitik keynesianischer Provenienz steht nur zur Disposition, wenn die tatsächliche Produktion unterhalb der Normalauslastung des Produktionspotentials liegt. Wir gehen aus diesem Grund in unserer Wirkungsanalyse stets von Unterbeschäftigung aus - auch deshalb, weil nach Meinung der Keynesianer eine derartige gesamtwirtschaftliche Konstellation eine vergleichsweise günstige Voraus-

1) Den gegenwärtigen Stand der Diskussion referieren Siebke, J., Knoll, D., Schmidberger, W.D., Theoretische Grundlagen des Crowding Out Effektes, in: Ehrlicher, $W$. (Hrsg.), Geldpolitik, Zins und Staatsverschuldung, Schriften des Vereins für Socialpolitik, Band 111, 1981, S. $231 \mathrm{ff}$. 
setzung dafür ist, daB eine Verdrängung privater Ausgaben vermieden wird. Von den Verdrängungsmechanismen interessieren mithin nur solche, welche bei Unterbeschäftigung wirksam sind.

b) Nachfrageseitige und angebotsseitige Ursachen von Unterbeschäftigung

Die Gründe, warum das Produktionspotential nicht in "normalem" AusmaB genutzt wird, können mit dem Sachverständigenrat in nachfrageseitige und angebotsseitige unterschieden werden ${ }^{1 !}$ ?

Nachfrageseitige Ursachen haben ihren Ursprung bei der Verwendung der Produktion; sie wurzeln in einem Güternachfragemangel und führen dazu, daB "die summe der in der Volkswirtschaft wirksam werdenden Ansprüche an das Produktionsergebnis kleiner ist als die akute oder potentielle Produktion, obwohl die individuellen Ansprüche an das Produktionsergebnis mittelfristig gesehen marktgerecht sind"2) Die produktion wurde ausgedehnt werden, wenn nur mehr Güter von privaten Konsumenten und Investoren sowie vom staat nachgefragt würden; denn den Unternehmern reichen die Grenzerlöse aus, um die fremden Ansprüche (von Arbeitnehmern, Fremdkapitalgebern sowie dem Staat) und auch die eigenen (zur Abdeckung des Unternehmerlohnes,

1) Vgl. hierzu die Gutachten des Sachverständigenrates seit 1976, insbesondere: Sachverständigenrat zur Begutachtung der gesamtwirtschaftlichen Entwicklung, Jahresgutachten 1976/77, Bundestagsdrucksache 7/5902, Ziff. 272 ff.; derselbe, Mehr Wachstum, mehr Beschäftigung, Jahresgutachten 1977/78, Stuttgart, Mainz.1977, Ziff. $236 \mathrm{ff.;}$ derselbe, Jahresqutachten 1978/79, Bundestagsdrucksache $8 / 2313$, Ziff. 266 ff.; derselbe, Jahresgutachten 1980/81, Bundestagsdrucksache 9/17, Ziff. 495 ff.; vgl. auch Sievert, O., Die Steuerbarkeit der Konjunktur durch den Staat, in: Weizsäcker, C.C. von (Hrsg.), Staat und wirtschaft, Schriften des Vereins für Socialpolitik, Band 102, Berlin,1979, S. $810 \mathrm{ff}$.

2) Sievert, O., Zur Steuerbarkeit der Konjunktur durch den staat, a.a.0., s. 821 . 
der Verzinsung des Eigenkapitaleinsatzes und einer Risikoprämie) zu befriedigen.

Angebotsseitige Störimpulse nehmen dagegen ihren Ausgang bei der Verteilung des Ergebnisses der Produktion und haben zum Resultat, daB "die summe der zum zuge kommenden Ansprüche an das Produktionsergebnis kleiner ist als die akute oder potentielle Produktion, weil die individuellen Ansprüche an das produktionsergebnis $\mathrm{zu}$ hoch sind"1) Die Ursache für eine angebotsseitig bedingte Unterbeschäftigung liegt mithin im streit um die Verteilung des Produktionsergebnisses. Dieser hat dann die fatale Konsequenz, daB Verteilbares gar nicht erst entsteht, wenn denjenigen, die darüber entscheiden, ob produziert wird oder nicht, ob sich also ein verteilbares produktionsergebnis ergibt oder nicht, die Befriedigung ihrer eigenen Ansprüche nicht zugestanden wird ${ }^{2}$ ! Die Alternative, das Produktionsvorhaben $\mathrm{zu}$ unterlassen, erscheint den Unternehmen dann vorteilhaft. Auch wenn die Nachfrage zunimmt, wird deshalb die produktion nicht ausgedehnt ${ }^{3}$ !

1) Sievert, 0., zur Steuerbarkeit der Konjunktur durch den staat, a.a.0., s. 821 .

2) "Die Situation der Angebotspolitik beruht auf Irrtümern in bezug auf die mit dem gesamtwirtschaftlichen Gleichgewicht $z u$ vereinbarenden Ansprüche an das Produktionspotential bzw. in bezug auf die bei gegebenen Ansprüchen rentablen Produktionsmöglichkeiten". Ebenda, S. 822 .

3) Vgl. auch derselbe, Arbeitslosigkeit und Lohnhöhe, in: Seidel, H., Butscheck, F. (Hrsg.), Ist Arbeitslosigkeit unvermeidlich?, Wien, 1979, S. 13 ff.; derselbe, Position des Sachverständigenrates, in: Vierteljahreshefte zur Wirtschaftsforschung, 1980, S. $18 \mathrm{ff}$. sowie Brenner, G. , Lipp, E.M., Beschäftigungspolitische Verantwortung der Finanzpolitik. Probleme bei ambivalenter Ursachendiagnose, Diskussionsbeiträge des Fachbereichs Wirtschaftswissenschaft der Universität des Saarlandes, Nr. A 7802, Juni 1978, S. 1 ff.; Lipp, E.M., Staatlicher Handlungsbedarf bei anhaltender Arbeitslosigkeit - Bemerkungen $z u$ einer anhaltenden Kontroverse, in: Public Finance, 1980, S. $155 \mathrm{ff}$.; Peston, M., An Aspect of the Crowding Out Problem, in: Oxford Economic Papers, 1981, S. $19 \mathrm{ff}$. 
DaB vom Symptom her gesehen gleiche gesamtwirtschaftliche Ausgangslagen unglelche Bestimmungsgründe haben können, spielt für ein crowding-out in Unterbeschäftigungslagen bei vielen Verdrăngungsmechanismen keine Rolle. Es können deshalb im wesentlichen nachfrageseitige Störimpulse als Gründe für eine Unterbeschäftigungslage unterstellt werden, also keynesianische Probleme, zu denen die keynesianische Rezeptur prima vista paBt. Davon gibt es zwel Ausnahmen: Bei angebotsseitigen Störimpulsen und einem expansiven finanzwirtschaftlichen demand management entfallt zum einen der von Keynesianern erwartete Akzelerationseffekt, und es ergeben sich zum anderen Preisstelgerungstendenzen, wenn zusätzliche Güternachfrage auf ein unelastisches Angebot trifft.

\section{Effekt}

1. Verdrängungsindikator: Privatausgaben

Ein crowding-out wird in unserer Wirkungsanalyse durch die insgesamt von den Privaten entfaltete Güternachfrage angezeigt. Dies ist zwar die im Schrifttum übliche, gleichwohl nicht durchweg praktizierte Vorgehensweise ${ }^{1 !}$ ?

Manchmal wird nur ein Teil der privaten Güternachfrage, nämlich der investive, als Verdrängungsindikator verwen$\operatorname{det}^{2}$ ! Zum einen hat dies einen historischen Grund. In

1) Vgl. z.B. Spencer, R.W., Yohe, W.P., The "Crowding Out" of Private Expenditures by Fiscal Policy Actions, a.a.o., S. $429 \mathrm{ff.;}$ Carlson, M.K., Spencer, R.W., Crowding-Out and its Critics, a.a.O., S. 2 ff.; Dieckheuer, G., Der Crowding-out-Effekt - zum gegenwärtigen Stand von Theorie und Empirie, a.a.0., S. 126 ff.

2) Vgl. z.B. Cebula, R.J., An Empirical Analysis of the "Crowding Out" Effect of Fiscal Policy in the United States and Canada, in: Kyklos, 1978, S. 424 ff.;

Cebula, R.J., Carlos, C., Koch, J.V., The "Crowding Out" Effect of Federal Government Outlay Decisions:

An Empirical Note, in: Public Choice, 1981, S. 329 ff. 
der Anfangsphase der crowding-out-Debatte konzentrierte sich das Interesse allein auf den $\mathrm{zins}$ als Transmissionsvariable eines crowding-out und auf die Verdrängung der vor allem vom $Z$ ins abhängenden privaten Investitionsausgaben. Häufig, insbesondere unter Praktikern, wird auch deswegen nicht der Gutermarkt, sondern der Kreditmarkt als ort der Verdrängung angesehen; zurückgedrängt wird private Kreditnachfrage (zur Finanzierung von Investitionen) durch staatliche 1) Zum anderen soll durch die Beschränkung auf die privaten Investitionen der Blick auf eine mögliche unerwünschte langfristige Nebenwirkung einer kurzfristig orientierten finanzwirtschaftlichen Stabilisierungspolitik gelenkt werden, nämlich Wachstumsverluste beim privaten Realkapital.

Uberwiegend (und auch hier) fungieren dagegen die gesamten Ausgaben der Privaten als Effektvariable. Dann interessiert nicht eine langfristige Nebenwirkung, sondern die beabsichtigte Hauptwirkung eines finanzwirtschaftlichen demand management bei Unterbeschäftigung: die Anhebung von Produktion und Beschäftigung in der Gegenwart. Denn für die aktuelle Auslastung des Produktionspotentials kommt es weniger auf die struktur, sondern vor allem auf das Niveau der insgesamt entfalteten

1) Vgl. z.B. Gandenberger, O., రffentliche Verschuldung II. Theoretische Grundlagen, in: Albers, W. u.a. (Hrsg.), Handwörterbuch der Wirtschaftswissenschaft, 5. Band, Stuttgart, Tübingen, Göttingen, 1980, s. 480 ff.; Kern, H., Crowding-out durch Staatsverschuldung?, in: Wirtschaftsdienst, 1980 , S. 566 ff.; Schips, B., Jäger, F., రkonomische Analyse der crowding-out-Wirkungen der Staatsverschuldung in der Schweiz, Vortrag, gehalten auf der Tagung Geld, Banken und Versicherungen in Karlsruhe vom 11.-13.12.1980; Frohwein, D.-K., Staatsverschuldung und Kapitalmarkt, Vortrag vom 17.1.1980, s. $24 \mathrm{ff}$. 
Güternachfrage an '? Crowding-out bedeutet demnach, daB die aktuelle Produktion und Beschäftigung zum Zweck der Bedienung privater Ausgabenwünsche geringer ist, als sie ohne stabilisierungspolitisch motivierte Staatsintervention wäre; der Verdrängungsindikator sind die gesamten Privatausgaben.

2. Zur "Null-Linie" von crowding-out

Die Scheidelinie zwischen einem crowding-out und einem pulling-in infolge einer finanzwirtschaftlichen MaBnahme ist das Niveau der privaten Ausgaben, welches bei $\mathrm{Ab}-$ wesenheit einer staatlichen Intervention getätigt worden wäre. Mit dieser Abgrenzung ist durchaus vereinbar, daB ein crowding-out auftritt und sich dennoch im Vergleich zur Vorperiode die private Güternachfrage erhöht ${ }^{2}$ !

1) $\mathrm{Ob}$ sich allerdings im Zuge eines Anstiegs der realen Güternachfrage des Staates (Gr) sowie der privaten Konsumenten $\left(C^{r}\right)$ und der Investoren $\left(I^{r}\right)$ tatsächlich die Beschäftigungsquote $(A B / A)$, gemessen als Anteil der abhängig beschäftigten Arbeitskräfte (AB). an der Zahl der abhängigen Erwerbspersonen (A) erhöht, hängt in der Realität zudem davon $a b$, wie sich in der betrachteten Periode die Zahl der abhängigen Erwerbspersonen, die durchschnittliche Arbeitszeit pro abhängig Beschäftigten ( $A Z / A B$ ) sowie die Arbeitsstundenproduktivität $\left(Y^{r} / \mathrm{AZ}\right)$ entwickeln. Der $\mathrm{Zu}-$ sammenhang zwischen der Beschäftigungsquote und den beiden genannten Gruppen von Bestimmungsfaktoren kommt in folgender Identitätsgleichung zum Ausdruck:

$$
\frac{A B}{A}=\frac{G^{r}+I^{r}+C^{r}}{A \cdot(A Z / A B) \cdot\left(Y^{r} / A Z\right)}
$$

In der Bundesrepublik Deutschland sind die realen Gesamtausgaben in den letzten Jahren stets gestiegen. Dennoch blieb die Beschäftigungsquote nahezu unverändert. Offensichtlich hat sich das Produkt der Bestimmungsfaktoren im Nenner gleichgerichtet und damit kompensierend verändert. Vgl. hierzu auch Münnich, F.E., Ist das Nachfragemanagement überholt?, Koreferat, in: Seldel, H., Butschek, F. (Hrsg.), Ist Arbeitslosigkeit unvermeidlich?, Wien, 1979, S. $104 \mathrm{f}$.

2) Vgl. Pohl, Reinhard, Einige geldtheoretische und geldpolitische Probleme des crowding-out des privaten sektors durch den staat, a.a.0., S. 264. 
In einer evolutorischen Wirtschaft wachsen die Ausgaben der Privaten von Periode zu Periode. Bei einer Zunahme um konstante Beträge $[\mathrm{d}(\mathrm{C}+\mathrm{I})=$ konstant $]$ kann schon dann von einem crowding-out gesprochen werden, wenn wegen des staatlichen Handelns der Zuwachs der Privatausgaben abnimmt:

$(1-4-10) \quad d^{\prime}(C+I)<0$.

Erhöhen sich die Privatausgaben um eine konstante Rate $\left(w^{C+1}=\right.$ konstant), führen finanzwirtschaftliche Impulse dann zu einem crowding-out, wenn sich die Wachstumsrate der privaten Güternachfrage verringert:

$$
(1-4-11) \quad d w^{C+I}<0
$$

Wir unterstellen aus Vereinfachungsgründen eine stationäre Wirtschaft, in der ebenso viele Privatausgaben wie in der Vorperiode getätigt worden wären, wenn der staat nicht stabilisierungspolitisch gehandelt hätte. Die "Null-Linie" ist in diesem Fall ein konstantes Niveau der Privatausgaben $(C+I=$ konstant $)$, und ein crowding-out erfolgt dann, wenn der staat interveniert und die Ausgaben der Privaten deshalb unter dieses Niveau sinkt:

$$
(1-4-12) \quad d(C+I)<0 \text {. }
$$

3. Crowding-out-Effekt als Gesamt- und als Teileffekt

Mit crowding-out-Effekt sind hier und in der Literatur zwei unterschiedliche Sachverhalte gemeint. Als crowdingout-Effekt bezeichnet man zum einen die insgesamt bewirkte Verminderung der privaten Ausgaben unter das Niveau, welches getätigt worden wäre, hätte sich der staat stabilisierungspolitisch enthalten. Dies ist ein crowding-out-Gesamteffekt. 
Finanzwirtschaftliches Handeln und die Privatausgaben sind uber eine Vielzahl von Transmissionskanălen miteinander verknüpft. Dabel wirken die einzelnen Primär- und Sekundärimpulse für sich genommen anregend oder dämpfend auf die privaten Aktivitäten. Elne ausgabenmindernde Wirkung eines einzelnen Primär- und Sekundărimpulses bezeichnet man in der crowding-out-Diskussion ebenfalls als crowding-outEffekt. Es handelt sich dabei aber nur um einen Teileffekt.

Steigen zum Beispiel im Zuge einer finanzwirtschaftlichen Maßnahme die Zinsen, so verringern sich die zinsabhängigen Privatausgaben; dies ist ein crowding-outTeileffekt. Werden alle crowding-out-Teileffekte addiert und überwiegen sie gegenüber den pulling-in-Teileffekten, dann hat die staatliche Aktivität auch einen crowding-outGesamteffekt zur Folge ${ }^{1)}$. Crowding-out-Gesamteffekt und crowding-out-Teileffekt unterscheiden sich mithin durch die verwendete BezugsgröBe. Bei einem Teileffekt ist es ein einzelner Primär- oder Sekundärimpuls, beim Gesamteffekt die finanzwirtschaftliche Maßnahme, d.h. die Summe der Primärimpulse.

D. Finanzwirtschaftliches demand management im Grundmodell

1. Grundmodell

Der Wirkungszusammenhang zwischen staatlicher Güternachfrage und privater Güternachfrage wird zunächst anhand eines einfachen (keynesianischen) Modells vom IS-LM-Typ dargestellt. Es weicht von der Standardversion nur dadurch $a b, d a B$ eine staatliche Finanzierungsrestriktion hinzugefügt wird. Dieses

1) Keynes war sich, wie erwähnt, durchaus bewußt, daB die stabilisierungspolitische Wirksamkeit eines finanzwirtschaftlichen demand management durch crowding-out-Teileffekte z.B. in Form eines Verlustes an Zukunftsvertrauen abgeschwächt werden kann; gleichwohl schlob er einen crowding-out-Gesamteffekt aus. 
Modell dient als Grundmodell. In seiner strukturellen Form besteht es aus 12 Gleichungen und 12 Variablen (vgl. Ubersicht 5): gesamtwirtschaftliches Guterangebot $\left(Y^{s}\right)$, gesamtwirtschaftliche Güternachfrage $\left(Y^{d}\right)$, realisiertes Sozialprodukt (Y), private Konsumnachfrage (C), private Investitionsnachfrage (I), verfügbares Einkommen $\left(Y^{V}\right),($ Netto-) Steueraufkommen $(T), Z$ ins (i), staatliche Güternachfrage (G), Zentralbankgeldangebot $\left(\mathrm{MZ}^{\mathrm{s}}\right)$, Geldnachfrage (L), tatsächliche Zentralbankgeldmenge (MZ) und Staatsschuldtitelbestand bei den Privaten (B).

Ubersicht 5: Das Grundmodell in seiner strukturellen Form Gleichung partielle
Ableitung Bezeichnung

$$
\begin{aligned}
& (1-4-13) \quad Y^{S}=Y^{d} \\
& (1-4-14) \quad Y=Y^{S}=Y^{d} \\
& (1-1-1) \quad Y^{d}=C+I+G
\end{aligned}
$$

$$
\begin{array}{lll}
(1-4-15) & \mathrm{C}=\mathrm{C}\left(\mathrm{Y}^{\mathrm{V}}\right) & 0<\mathrm{C}_{\mathrm{Y}}<1 \\
(1-4-16) & \mathrm{Y}^{\mathrm{V}}=\mathrm{Y}-\mathrm{T} &
\end{array}
$$

Gleichgewichtsbedingung für den Gütermarkt Definition des realisierten Sozialprodukts Gesamtwirtschaftliche Güternachfragefunktion Konsumfunktion Definitionsgleichung für das verfügbare Einkommen

$$
(1-4-17) \quad \mathrm{T}=\mathrm{T}(\mathrm{Y}) \quad 0<\mathrm{T}_{\mathrm{Y}}<1
$$

Steueraufkommensfunktion

1) $\mathrm{zu}$ einer Funktion $(\mathrm{x}, \mathrm{y}, \ldots, \mathrm{z})$ wird im folgenden für die partielle Ableitung die Schreibweise $\partial \mathrm{f} / \partial \mathrm{x}=\mathrm{f}_{\mathrm{x}}$ verwendet. 


$$
\begin{array}{ll}
(1-4-18) & I=I(i) \quad I_{i}<0 \\
(1-4-19) & G=\bar{G} \\
(1-4-20) & M Z^{S}=L \\
(1-4-21) \quad \mathrm{MZ}^{S}=\overline{M Z}^{S} \\
(1-4-22) \quad \mathrm{L}=\mathrm{L}(\mathrm{Y}, \mathrm{i}) \quad \mathrm{L}_{i}<0 ; L_{Y}>0 \\
(1-4-23) \quad \mathrm{MZ}=\mathrm{L}=\mathrm{MZ}^{S} \\
(1-4-24) \quad \mathrm{G}=\mathrm{T}+\mathrm{B}-\bar{B}_{-1}
\end{array}
$$

Investitionsfunktion

Transformationsausgabenfunktion

Gleichgewichtsbedingung für den Geldmarkt

Zentralbankgeldangebotsfunktion

Geldnachfragefunktion

Definition der tatsächlichen Zentralbankgeldmenge

Staatliche Finanzierungsrestriktion

Werden die Verhaltensgleichungen in die Gleichgewichtsbedingungen für den Güter- und Geldmarkt sowie in die staatliche Finanzierungsrestriktion eingesetzt, und werden die Definitionen für das realisierte Sozialprodukt und für die tatsächliche Zentralbankgeldmenge berücksichtigt, ergibt sich als semi-reduzierte Form:

$$
\begin{aligned}
(1-4-25) & Y-C(Y-T)-I(i)-\bar{G} & =0, \\
(1-4-26) & L(Y, i)-\overline{M Z} & =0, \\
(1-4-27) & \bar{G}-T(Y) & =B-\bar{B}
\end{aligned}
$$

Die Gleichungen (1-4-25) und (1-4-26) stellen in einem i-Y-Koordinatensystem die hinlänglich bekannten IS- und LM-Kurven - im folgenden allerdings linearisiert - dar. Die Gleichung (1-4-27) bringt die staatliche Finanzierungsrestriktion zum Ausdruck. 


\section{Wirkungsanalyse}

a) Gesamtausgabeneffekt

Um den Gesamtausgabeneffekt einer kreditmarktfinanzierten Erhöhung der Transformationsausgaben $z u$ ermitteln, bilden wir das totale Differential:

$$
\begin{array}{lll}
(1-4-28) & d Y-C_{Y} \cdot\left(1-T_{Y}\right) d Y-I_{i} d i-d \bar{G} & =0, \\
(1-4-29) & I_{Y} d Y+L_{i} d i-d \overline{M Z} & =0, \\
(1-4-30) d \bar{G}-T_{Y} d Y & =d B .
\end{array}
$$

Von den drei Politikvariablen der staatlichen Finanzierungsrestriktion $(1-4-24)\left(G, T, B-\bar{B}_{1}\right)$ ist das Steueraufkommen einkommensabhängig; zudem ist wegen der Beschränkung der Handlungsfreiheit finanzpolitischer Entscheidungsträger durch die staatliche Finanzierungsrestriktion von den zwei verbleibenden Politikvariablen eine weitere endogenisiert $^{1)}$ : im Fall einer autonomen Erhöhung der Transformationsausgaben folglich die Änderung des Staatspapierbestandes bei den Privaten. Zusammen mit der Zins- und Sozialproduktsveränderung ist die Staatspapierbestandsvariation eine der drei schlüsselvariablen des Gleichungssystems $(1-4-28)$ bis $(1-4-30)$; es lautet in Matrixschreibweise:

$(1-4-31)\left[\begin{array}{ccc}1-\mathrm{C}_{\mathrm{Y}} \cdot\left(1-\mathrm{T}_{\mathrm{Y}}\right) & -\mathrm{I}_{\mathrm{i}} & 0 \\ \mathrm{~L}_{\mathrm{Y}} & \mathrm{L}_{\mathrm{i}} & 0 \\ \mathrm{~T}_{\mathrm{Y}} & 0 & 1\end{array}\right] \cdot\left[\begin{array}{c}\mathrm{dY} \\ \mathrm{di} \\ \mathrm{dB}\end{array}\right]=\left[\begin{array}{c}\mathrm{d \overline {G }} \\ 0 \\ \mathrm{~d} \overline{\mathrm{G}}\end{array}\right]$.

1) Vgl. Christ, C.F., A Simple Macroeconomic Model with a Government Budget Restraint, a.a.0., S. 55 f. 
Als Gesamtausgabenmultiplikator einer Erhöhung der Transmissionsausgaben, welche auf dem Kreditmarkt finanziert wird, ergibt sich nach der Cramer-Regel:

$$
(1-4-32) \quad \frac{d Y}{d \bar{G}}=\frac{1}{1-C_{Y} v\left(1-T_{Y}\right)+\frac{I_{1}}{L_{1}} L_{Y}}
$$

In der graphischen Darstellung (vgl. Abb. 1) wird durch eine Ausdehnung der Güternachfrage des Staates, die durch Emission von Staatsschuldtiteln finanziert wird, die IS-Kurve nach rechts von Is ${ }_{0}$ aus IS ${ }_{1}$ verschoben. Den Gesamtausgabeneffekt kann man als Differenz zwischen den gleichgewichtigen Sozialprodukten vor und nach der finanzwirtschaftlichen Maßnahme ablesen $\left(Y_{1} \longrightarrow Y_{3}\right)$.

b) Privatausgabeneffekte

Der Privatausgabenmultiplikator hat einen Wert von:

$$
\begin{aligned}
& (1-4-33) \frac{d(C+I)}{d \bar{G}}= \\
& \underbrace{1-\mathrm{C}_{\mathrm{Y}} \cdot\left(1-\mathrm{T}_{\mathrm{Y}}\right)}_{\begin{array}{l}
\text { einkommens- } \\
\text { induzierter } \\
\text { Konsumeffekt }
\end{array}}+\underbrace{\underbrace{\mathrm{I}_{\mathrm{i}} \mathrm{L}_{\mathrm{Y}}}_{\mathrm{I}_{\mathrm{i}}}}_{\begin{array}{l}
\text { Hicks- } \\
\text { scher } \\
\text { crowding-out- } \\
\text { Effekt }
\end{array}}-1
\end{aligned}
$$

Der Privatausgabengesamteffekt ist das Produkt aus dem Privatausgabenmultiplikator und den zusätzlichen staatlichen Ausgaben.

Aus der Menge möglicher Privatausgabenteileffekte infolge einer kreditmarktfinanzierten Vermehrung der Transformationsausgaben werden im Grundmodell nur zwei 
Abbildung 1: Gesamtausgabeneffekt und Privatausgabeneffekte im Grundmodell

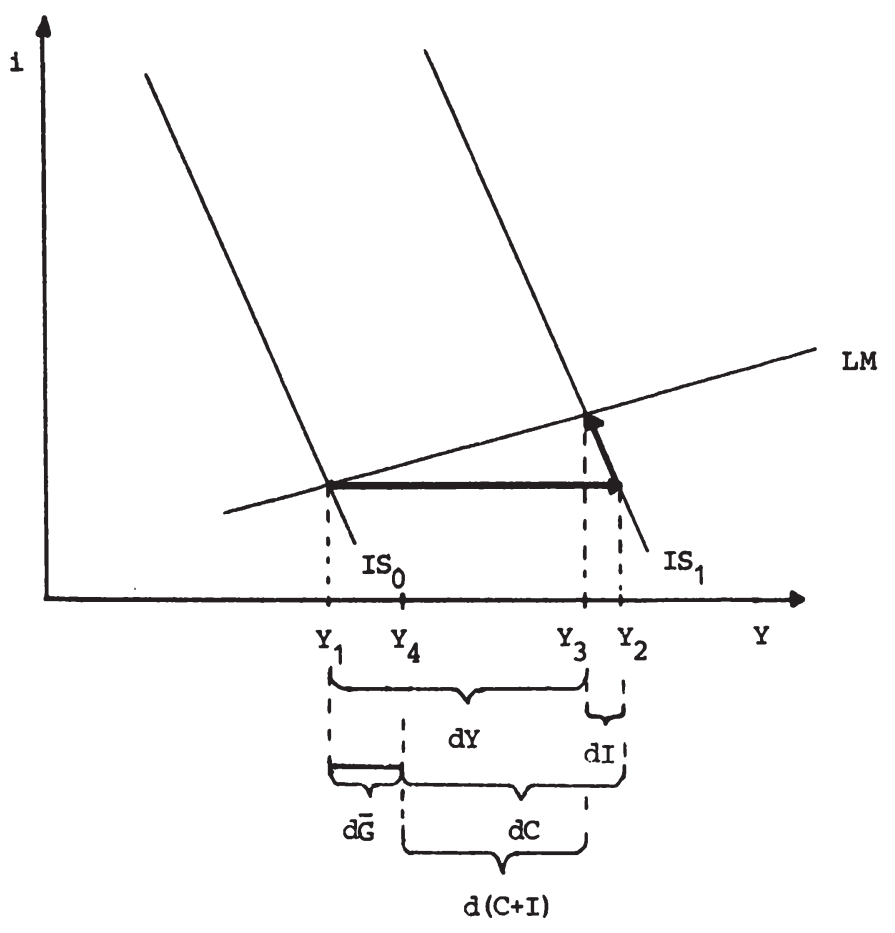

erfaBt [vgl. Gleichung $(1-4-33)]^{1 !}$

(1) Der einkommensinduzierte Konsumeffekt: Eine Erhohung der Transformationsausgaben fuhrt be 1 den Empfängern dieser Ausgaben zu zusătzlichem verfügbaren Einkommen, was diese dazu veranlaBt, nach MaBgabe ihrer marginalen Konsumquote mehr $\mathrm{zu}$ verbrauchen. Dies vermehrt das verfügbare Einkommen anderer, welche ihrerseits den Konsum ausdehnen und so fort.

1) Vgl. Modigliani, F., Ando, A., Impacts of Fiscal Actions on Aggregate Income and the Monetarist Controversy: Theory and Evidence, in: Stein, J.L. (Hrsg.), Monetarism, Amsterdam, New York, Oxford, 1976, S. $23 \mathrm{f}$. 
(2) Der Hickssche crowding-out-Effekt: Der mit einer Gesamtausgabenerhöhung einhergehende Mehrbedarf an Transaktionskasse kann bei gegebener Geldmenge nur durch eine Verringerung der aus spekulativen Gründen gehaltenen Kassenbestände befriedigt werden. Im Zuge der Aktivierung von "idle money" steigen in Abhängigkeit von der zinselastizität der Geldnachfrage die zinsen und dämpfen die private Investitionstätigkeit.

In der graphischen Darstellung (vgl. Abb. 1) stellt die Bewegung auf der IS-Kurve von $\mathrm{Y}_{2}$ nach $\mathrm{Y}_{3}$ den Hicksschen crowding-out-Effekt dar. Um den Unterschiedsbetrag zwischen $\mathrm{Y}_{2}$ und $\mathrm{Y}_{3}$ sinken die privaten Investitionen (dI), weil infolge der staatlichen Aktivität der Bedarf an Transaktionskasse zunimmt und die Zinsen steigen. Der andere Privatausgabenteileffekt, der einkommensinduzierte Konsumeffekte, kommt in der Rechtsverschiebung der IS-Kurve von IS 0 auf IS ${ }_{1}$ zum Ausdruck: Zusammen mit den staatlichen Zusatzausgaben bestimmt er deren AusmaB, also den Abstand zwischen $Y_{1}$ und $Y_{2}$. Zieht man davon die zusätzlichen Transformationsausgaben $a b\left(z \cdot B .: d \bar{G}=\overline{Y_{1} Y_{4}}\right)$, erhält man als Restbetrag den einkommensinduzierten Konsumeffekt (dC).

Der Saldo aus den beiden Privatausgabenteileffekten gibt den Privatausgabengesamteffekt an $\left[\mathrm{d}(\mathrm{C}+\mathrm{I})=\overline{\mathrm{Y}_{4} \mathrm{Y}_{3}}\right]$. Liegt $\mathrm{Y}_{3}$ rechts von $\mathrm{Y}_{4}$, wie in $\mathrm{Abb}$. 1, werden durch die staatlichen Ausgaben die Privatausgaben insgesamt angeregt, der Privatausgabenmultiplikator hat also einen positiven Wert, und es wird ein pulling-in-Gesamteffekt bewirkt. Im umgekehrten Fall, wenn sich $Y_{3}$ links von $Y_{4}$ befindet, werden durch die finanzwirtschaftlichen Aktivitäten die Privatausgaben in summa gedämpft, der Privatausgabenmultiplikator hat mithin einen negativen Wert, es kommt zu einem crowding-out-Gesamteffekt. Es handelt sich dabei um ein vollständiges crowding-out, wenn sich $\mathrm{Y}_{3}$ und $\mathrm{Y}_{1}$ entsprechen: Um den Betrag der zusätzlichen Transformationsausgaben gehen die Privatausgaben zurück. 


\section{Zur keynesianischen Position}

Damit im Grundmodell ein crowding-out-Gesamteffekt vermieden wird, darf der Hickssche crowding-out-Effekt den einkommensinduzierten Konsumeffekt nicht überkompensieren. Genau dies nehmen Keynesianer für Unterbeschäftigungslagen an ${ }^{1)}$. Denn zum einen ist nach ihrer Meinung die Neigung der Privaten groB, aus zusätzlich verfügbarem Einkommen zu konsumieren. Zum anderen sei gerade dann, wenn die aktuelle Produktion unter der potentiellen liegt, die zinselastizität der privaten Investitionen (absolut) gering und die der Geldnachfrage (absolut) groB.

Im IS-LM-Schema ist unter diesen Annahmen, die auch der $\mathrm{Abb}$. 1 zugrundeliegen, die LM-Kurve ziemlich flach, die ISKurve relativ steil, und die Rechtsverschiebung der IS-Kurve fällt vergleichsweise stark aus. In $\mathrm{Abb}$. 1 wird mithin nicht nur das Grundmodell graphisch dargestellt, sondern es kommt darin zugleich in einfacher Form die keynesianische Position zum Ausdruck: Die Privatausgaben erhöhen sich (um ein Mehrfaches des staatlichen Nachfrageimpulses), wenn der staat zusätzliche Ausgaben tätigt.

Die Monetaristen sind, wie gesagt, gegenteiliger Auffassung. Ergreift der Staat Ankurbelungsmaßnahmen, kommt es nach ihrer Ansicht $z u$ keinem pulling-in, sondern $z u$ einem mehr oder minder vollständigen crowding-out: Das Niveau der Privatausgaben sinkt, und zwar um etwa den Betrag des staatlichen Nachfrageimpulses. Ausgehend von der keynesianischen Position im Grundmodell kann die monetaristische These auf zwei Wegen begründet werden: zum einen damit, daß für die beiden genannten Privatausgabenteileffekte andere Werte, als von Keynesianern vermutet, angenommen werden und zum anderen damit, daß zusätzliche Dämpfungseffekte berücksichtigt werden. Im folgenden zweiten Teil werden beide Wege beschritten.

1) Vgl. z.B. Vane, H.R., Thompson, J.L., Monetarism. Theory, Evidence and Policy, Oxford, 1979, S. 19 ff. 
Zweiter Teil: Zu den Ursachen des crowding-out

Erstes Kapitel: Der Hickssche crowding-out-Effekt

Monetaristen gehen davon aus, daB die zinselastizitat der Geldnachfrage und die der privaten Güternachfrage für die stabilisierungspolitische wirksamkeit finanzwirtschaftlicher MaBnahmen vergleichsweise ungünstige Werte haben: Die Geldnachfrage ist ihrer Ansicht nach relativ zinsunelastisch und die private Güternachfrage ziemlich zinselastisch. Dadurch gewinnt das Hickssche crowding-out ${ }^{1)}$ an stärke, und finanzwirtschaftliche Stabilisierungsmaßnahmen verlieren an Effizienz ${ }^{2}$.

Ist gar die Zinselastizität der Geldnachfrage gleich Null, erfolgt ein vollständiges Hickssches crowdingout. Denn dann entfällt die bei einer annahmegemäB konstanten Geldmenge verbleibende Möglichkeit einer Finanzierung der zusätzlichen Ausgaben via einer Forcierung des Geldumlaufs. Die Gesamtausgaben verharren deshalb auf dem durch den monetären Rahmen beschränkten Niveau, und die private Guternachfrage sinkt um den Betrag der zusätzlichen Transformationsausgaben. Weil $L_{1}=0$ ist, nimmt also der Privatausgabenmultiplikator

1) Die Bezeichnung stammt von Modigliani, F., Ando, A., Impacts of Fiscal Actions on Aggregate Income and the Monetarist Controversy: Theory and Evidence, a.a.0., S. 24 ; gebräuchlich sind auch "transaction crowdingout" und "transaction drain" sowie "monetary crowdingout".

2) Vgl. Butkiewicz, J.L., Some Macroeconomic Effects of Government Debt, Diss., University Microfilms International, Ann Arbor, Michigan, 1977, S. 11 ff.; Friedman, B.M., Crowding Out or Crowding In? Economic Consequences of Financing Government Deficits, a.a.0., S. 599 ff.; Davidson, P., The Crowding Out Effect. Monetarists vs. Keynesians vs. Keynes, Paper presented at the $53 \mathrm{rd}$ Annual Meetings of the restern Economic Association Conference in Hawai, 1978, S. 1 ff.; Meyer, L.H., Financing Constraints and the Short-Run Response to Fiscal Policy, in: Federal Reserve Bank of St. Louis Review, 1980, Nr. 6, S. 27. 
$(1-4-33)$ einen Wert von $\left.\mathrm{d}(\mathrm{C}+\mathrm{I}) / \mathrm{d} \overline{\mathrm{G}}=-1 \mathrm{an}^{1}\right)$
$(\text { klassischer Fall })^{2}$ ?

Der klassische Fall wurde zu Beginn der Renaissance der crowding-out-These von vor allem Tobin in den Mittelpunkt der Diskussion gerückt. Er interpretierte beispielsweise zwei Aufsätze von Milton Friedman im Journal of Political Economy in den Jahren 1970 und $1971^{3)}$ dergestalt, daB die Annahme einer zinsunabhängigen Geldnachfrage hauptsächliches Kennzeichen der monetaristischen Position sei, woraus notwendigerweise crowding-out folgt: "First, let me explain what I thought the main issue was. In terms of the Hicksian language of Friedman's article, I thought (and I still think) it was the shape of the LM locus ... it will be vertical if the demand for money is wholly insensitive to interest rates. This assumption leads to the following characteristic monetarist propositions: ... a shift of the Is locus, whether due to fiscal policy or to exogenous change in consumption and investment behaviour cannot alter $Y^{\prime 4}$ !) In seiner Ent-

1) Buiter lastet diese Verdrängung nicht den finanz-, sondern den geldpolitischen Akteuren an: "The multiplier is reduced by a scarcity not of real resources (labour and capital), but of money, which pushes up interest rates when the economy begins to expand. This situation could therefore be better described as the crowding out of private spending by restrictive monetary policy than by public spending".

Buiter, W.H., The "Crowding Out" and the Effectiveness of Fiscal Policy, a.a.0., S. 313.

2) Vgl. Carlson, K.M., Spencer, R.W., Crowding Out and its Critics, a.a.0., s. $4 \mathrm{f}$.

3) Beide Aufsätze wurden später von Friedman zu einem zusammengefaBt und mit der sich daran anschlieBenden Diskussion noch einmal von R.J. Gordon veröffentlicht, vgl. Friedman, M., A Theoretical Framework for Monetary Analysis, in: Gordon, R.J. (Hrsg.), Milton Friedman's Monetary Framework. A Debate with his Critics, Chicago, London, 1974, S. $1 \mathrm{ff}$.

4) Tobin, J., Friedman's Theoretical Framework, a.a.o., S. $77 \mathrm{f}$. 
gegnung wies Milton Friedman diese Interpretation zurück und verwarf die Annahme einer zinsunabhängigen Geldnachfrage als allein konstitutiv für crowding-out. Vielmehr stellte er auch auf eine groBe zinselastizität der privaten Güternachfrage als Ursache für ein crowding-out $a b^{1 !}$ ?

Wenn die private Investitionsguternachfrage völlig zinselastisch ist, zeltigen expansive finanzwirtschaftliche Impulse - sofern der sich erhöhende Bedarf an Transaktionskasse nur zu steigenden zinsen befriedigt werden kann keinen aggregativen Effekt, auch wenn der monetäre Rahmen hinreicht (Knight Fall) 2 ) Der Privatausgabenmultiplikator (1-4-33) nimmt wiederum einen wert von $d(C+I) / d \bar{G}=-1$ an - jetzt allerdings, weil $I_{i}=-\infty$ ist.

Eine völlig zinselastische Investitionsgüternachfrage folgt aus der Knightschen Hypothese, die Grenzleistungsfähigkeit des Kapitals sei im relevanten Bereich konstant. Zum einen sei nämlich der Zuwachs zum Realkapitalstock durch Investitionen im Verglelch zum bereits existierenden Bestand sehr gering, und zum anderen wurden allfällige Verminderungstendenzen im zuge einer Realkapitalstockausdehnung durch den in den Investitionen inkorporierten technischen Fortschritt neutralisiert. Eine konstante Grenzleistungsfähigkeit des Kapitals Im relevanten Bereich veranlaBt Investoren, schon bei geringfügigen zinserhöhungen ihre Investitionsausgaben massiv einzuschränken.

Milton Friedman ging es jedoch weniger um die Zinselastizität der investiven, als vielmehr um die der konsumtiven Privatausgaben: "... the monetarist view is that ... the

1) Vgl. Friedman, M., Comments on the Critics, a.a.o., S. 137 ff.

2) Vgl. Carlson, K.M., Spencer, R.W., Crowding Out and its Critics, a.a.o., s. 6 f. 
categories of spending affected by changes in interest rates are far broader than the business capital formation ...." 1 ?

Dem Argument Milton Friedmans kann dadurch Rechnung getragen werden, daB der Zins als Bestimmungsfaktor des privaten Konsums berücksichtigt wird:

$$
(2-1-1) \quad C=C(Y-T, i) \quad \text { mit } C_{i}<0 \text {. }
$$

Sind neben den privaten Investitions- auch die privaten Konsumausgaben zinsabhängig, ergibt sich im derart modifizierten Grundmodell als Privatausgabenmultiplikator:

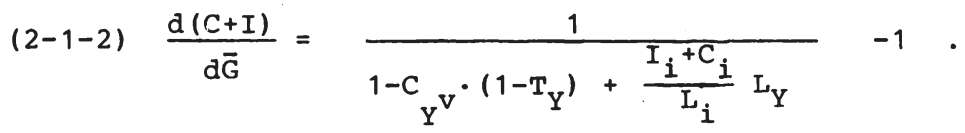

Er hat im Vergleich zum Privatausgabenmultiplikator (1-4-33) einen geringeren wert.

Ergebnis: Durch das Hickssche crowding-out wird die Wirksamkeit expansiver finanzwirtschaftlicher MaBnahmen zweifelsohne beeinträchtigt. Die EffizienzeinbuBe ist um so gröBer, je zinselastischer die private Güternachfrage ist und je zinsunelastischer die Geldnachfrage ist. Nach einer Schätzung von Benjamin Friedman 2), welche sich auf eigene empirische Untersuchungen sowie auf fremde (von Goldfeld und Hamburger) stützt, beläuft sich der Effizienzverlust aufgrund des Hicksschen crowding-out in der kurzen Frist auf etwa ein Zehntel (und in der langen Frist auf mehr als ein Drittel); wenn es keinen Hicksschen crowding-out-Effekt gäbe, wïrde

1) Friedman, M., Comments on the Critics, a.a.0., S. 140.

2) Vgl. Friedman, B.M., Crowding Out or Crowding In? Economic Consequences of Financing Government Deficits, a.a.0., S. $603 \mathrm{ff}$. 
eine Zunahme der Gesamtausgaben um 10 vom Hundert höher ausfallen.

Der für das Hickssche crowding-out verantwortliche Zinsauftrieb darf allerdings keineswegs mit dem zinseffekt infolge einer Kreditaufnahme des Staates verwechselt werden; er ist allein darauf zurückzuführen, daB sich im Zuge eines Expansionsprozesses das $z u$ bewältigende Transaktionsvolumen erhobt und deswegen die Nachfrage nach Zahlungsmitteln steigt. 
Zweites Kapitel: Vermögenseffekte

A. Das konventionelle Portfolio-crowding-out

1. Darstellung

a) Entstehungsphase des Defizits

Von monetaristischer Seite ist immer wieder kritisiert worden, daß der $\mathrm{Z}$ inserhöhung, welche mit einer Emission von Staatsschuldtiteln einhergehe, bei der Beurteilung der stabilisierungspolitischen Wirksamkeit einer kreditmarktfinanzierten Erhöhung der Transformationsausgaben zu wenig Beachtung geschenkt wird". Im Grundmodell, welches Keynesianer bevorzugt verwenden, wird der Zinseffekt einer staatspapieremission gar ignoriert.

Den $\mathrm{Z}$ inseffekt einer Emission von Staatsschuldtiteln machen Monetaristen regelmäßig mit Hilfe vermögenstheoretischer Uberlegungen plausibel ${ }^{2}$ ?

Erstens wird ein Zuwachs des Vermögens der Privaten konstatiert. Denn wie aus der Finanzierungsrestriktion des Staates hervorgeht, erhöht sich mit einer Ausdehnung der Staatsausgaben, insoweit diese nicht durch zusätzliche Steuereinnahmen finanziert wird, der Bestand an Staatspapieren im privaten Bereich. Bei Konstanz aller anderen Vermögenskomponenten steigt in diesem Umfang das

1) Vgl. z.B. Meyer, L.H., Hart, W.R., On the Effects of Fiscal and Monetary Policy: Comoleting the Taxonomy, in: American Economic Review, 1975, S. $762 \mathrm{ff}$.

2) Vgl. Meyer, L.H., A Balance Sheet Identity, the Government Financing Constraint, and the Crowding-Out Effect, in: Journal of Monetary Economics, 1975, S. $65 \mathrm{ff.;}$ Dieckheuer, G., Der Crowding-Out-Effekt - zum gegenwärtigen Stand von Theorie und Empirie, a.a.O., S. 133 ff.; vgl. auch Zahn, F., A Flow of Funds Analysis of Crowding Out, in: Southern Economic Journal, 1978, S. 136 ff. 
Vermögen der Privaten.

Zweitens wird davon ausgegangen, daB die Vermögenshalter stets eine Diversiflzierung ihres Vermögens unter Risikound vor allem Ertragsgesichtspunkten auf verschiedene Vermögenskomponenten anstreben. Bei sonst gleichen Bedingungen wird ein Vermögenszuwachs, der sich allein in einer Ausweitung des staatspapierbestandes manifestiert, als unbefriedigend empfunden; die Portfoliostruktur ist vergleichsweise $z u$ illiquide. Infolge der UberschuBnachfrage nach Geld setzt ein Reallokationsproze $B$ ein, im zuge dessen sich die zinsen nach Maßgabe der Zinselastizität der Geldnachfrage erhöhen, und der Wunsch nach zusätzlicher Kasse gedämpft wird. Die Zinsen steigen solange, bis die tatsächliche Portefeuillestruktur mit der geplanten übereinstimmt. Die mit der Umstrukturierung des Portefeuilles der Privaten verbundene $\mathrm{Zinserhöhung} \mathrm{drängt} \mathrm{private} \mathrm{zinsabhängige} \mathrm{Aus-}$ gaben zurück; dies ist das konventionelle Portfoliocrowding-out.

b) Reine Finanzierungsphase des Defizits

Dem konventionellen Portfolio-crowding-out wird von Monetaristen insbesondere auf mittlere sicht eine erhebliche Bedeutung beigemessen 1). Damit nämlich in den Folgeperioden dämpfende Staatsausgabenimpulse vermieden werden, muB die zusätzliche staatliche Güternachfrage aufrechterhalten werden. Denn eine Ausdehnung der staatlichen Ausgaben ist ein StromgröBenimpuls. Solche Impulse bewirken (sofern be 1 deren Transmission in den privaten Aktivitätsbereich nicht auch BestandsgröBen modifiziert werden) nur einmalige Privatausgabeneffekte - in der Regel in der Periode, in welcher sie ausgelöst werden. Entfallen sie in den folgenden Perioden, so bleiben auch die Wirkungen auf die Pri-

1) Vgl. Meyer, L.H., A Balance Sheet Identity, the Government Financing Constraint, and the Crowding-Out Effect, a.a.0., S. 76 f.; Dieckheuer, G., Möglichkeiten und Risiken einer kreditfinanzierten Stabilisierungspolitik, a.a.0., S. $559 \mathrm{ff}$. 
vatausgaben aus. Um die Ausgaben der Privaten dauerhaft auf dem erhöhten Niveau zu halten, muB in den Folgeperioden die zusätzliche Güternachfrage des staates weiterhin entfaltet werden.

Bei der Beschaffung der Mittel auf dem Kreditmarkt ist dies anders als bei der Mittelverwendung auf dem Gütermarkt. Denn uno actu mit einer einmaligen Kreditaufnahme des Staates erhöht sich auf Dauer der Bestand an Staatspapieren in den Portefeuilles der Privaten - und eine fortgesetzte Kreditaufnahme hat kumulative Staatsschuldbestandseffekte. In der reinen Finanzierungsphase des Defizits wird folglich nach Maßgabe des Umfangs des jeweils zu finanzierenden Haushaltsdefizits in jeder Haushaltsperiode erneut ein konventioneller Portfolioeffekt induziert.

Zusammengenommen: Mögen auch die pulling-in-Effekte in der Entstehungsphase des Defizits überwiegen, kann diese Anregung der privaten Ausgaben auf mittlere Frist wieder zunichte gemacht werden. Denn in der reinen Finanzierungsphase des Defizits wird von Seiten der Staatsausgaben im Vergleich zur jeweiligen Vorperiode kein Privatausgabeneffekt mehr induziert, wohl aber durch die Kreditaufnahme in jeder Periode erneut ein konventioneller Portfoliocrowding-out-Effekt.

2. Berücksichtigung im Grundmodell

a) Entstehungsphase des Defizits

Das konventionelle Portfolio-crowding-out läBt sich, wie dies SiJber ${ }^{1)}$ vorgeschlagen hat, im Grundmodell berücksichtigen. Dazu wird das Grundmodell wie folgt modi-

1) Vgl. Silber, W.L., Fiscal Policy in IS-LM-Analysis: A Correction, in: Journal of Money, Credit, and Banking, 1970, S. $461 \mathrm{ff}$. 
fiziert. In seiner strukturellen Form wird es um eine Gleichung erweitert, eine zweite wird verändert.

Hinzugefügt wird eine Definition des Vermögens des privaten sektors:

$(2-2-1) \quad V=B+M Z$.

Es besteht nur aus zwei Komponenten: Zentralbankgeld und staatsschuldtiteln. Diese Vermögensdefinition ist eng; sie entspricht einer häufig verwendeten Definition und wird noch kritisch gewürdigt"

Verändert wird die Geldnachfragefunktion. Das Vermögen wird als Determinante der Liquiditätspräferenz berücksichtigt. Damit werden die oben angestellten portfoliotheoretischen Uberlegungen in das Grundmodell integriert:

$(2-2-2) \quad L=L(Y, i, V) \quad$ mit $L_{V}>0$.

Werden die Modifikationen bei den einzelnen Rechenoperationen berücksichtigt, ergibt sich für die Entstehungsphase des Defizits als Privatausgabenmultiplikator:

$$
(2-2-3) \frac{d(C+I)}{d \bar{G}}=\frac{1-\frac{I_{i}}{L_{i}} L_{V}}{1-C_{Y} \cdot\left(1-T_{Y}\right)+\frac{I_{i}}{L_{i}}\left(L_{Y}-L_{V} T_{Y}\right)}-1 .
$$

Im Vergleich zur Gleichung (1-4-33) stellen wir zwei Unterschiede fest. Wichtig ist vor allem der zusätzliche Term im zähler des Multiplikators, welcher Ausdruck des konventionellen Portfolio-crowding-out ist. Hat dieser gar einen Wert von Eins, so wird der Zähler und auch der Bruch insgesamt gleich Null;

1) Vgl. unten S. $79 \mathrm{ff..}$ S. $89 \mathrm{ff}$. und S. $104 \mathrm{ff}$. 
Eine kreditmarktfinanzierte Ausdehnung der staatlichen Güternachfrage hat in diesem Fall schon in der Fntstehungsphase des Defizits ein vollständiges crowding-out zur Folge.

Im IS-LM-Diagramm kommt das konventionelle Portfolio-crowding-out in einer Linksverschiebung der LM-Funktion zum Ausdruck (vgl. Abb. 2): Infolge des Vermögensanstiegs entsteht ein verstärkter Bedarf an Kasse, welcher durch eine Einkommensverminderung und/oder einen 2 insanstieg abgebaut werden kann. $\mathrm{Zu}$ dem einkommensinduzierten Konsumeffekt $\left[\left(\mathrm{Y}_{1} \rightarrow \mathrm{Y}_{2}\right)-\mathrm{dG}\right]$ sowie dem Hicksschen crowding-out $\left(\mathrm{Y}_{2} \rightarrow \mathrm{Y}_{3}\right)$ tritt noch ein weiterer crowding-out-Teileffekt, nämlich das konventionelle Portfolio-crowding-out $\left(\mathrm{Y}_{3} \rightarrow \mathrm{Y}_{4}\right)$. Abbildung 2: Konventionelles Portfolio-crowding-out in der Entstehungsphase des Defizits

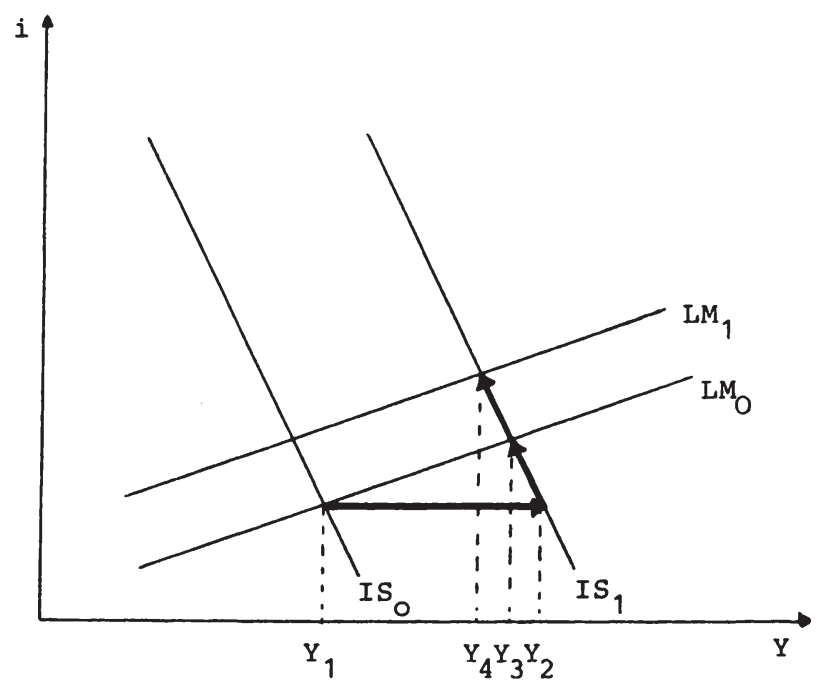


b) Reine Finanzierungsphase des Defizits

In der reinen Finanzierungsphase des Defizits werden Impulse nur noch durch die ständige Aufstockung des Bestandes an Staatspapieren bel den Privaten entfaltet. Dadurch verschiebt sich die LM-Kurve von Haushaltsperlode zu Haushaltsperiode weiter nach links. Die gleichgewichtigen Gesamtausgaben- und Privatausgabenniveaus sinken nach jedem neuerlichen deficitspending (vgl. $\mathrm{Abb}, 3)$.

Abbildung 3: Konventlonelles Portfolio-crowding-out in der reinen Finanzierungsphase des Defizits

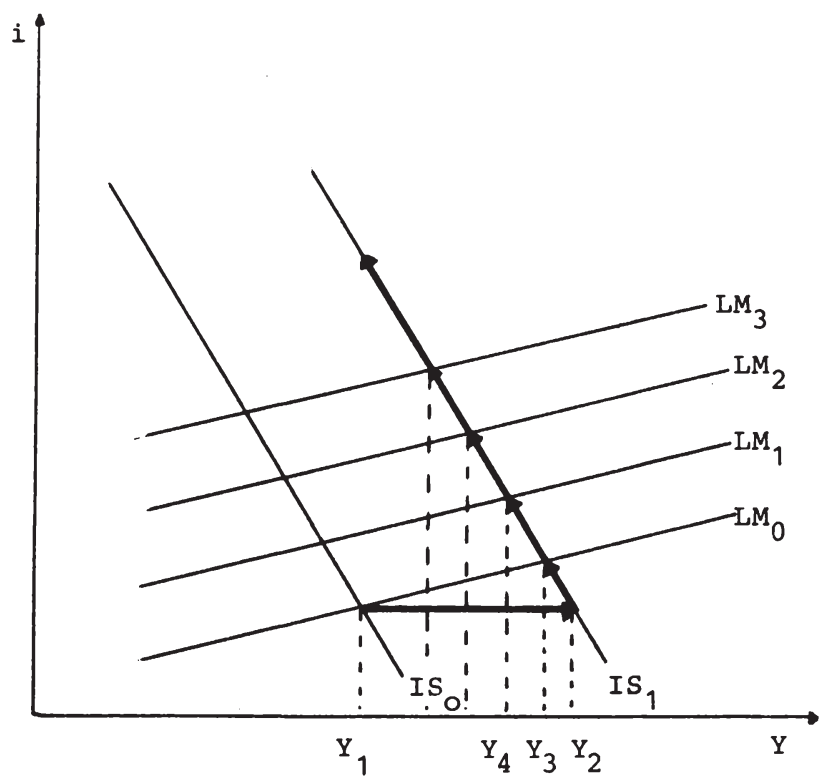


Nach monetaristischer Auffassung wird eine Rechtsverschiebung der IS-Kurve in der Entstehungsphase des Defizits auf mittlere Frist durch die Linksverschiebung der LM-Kurve in der reinen Finanzierungsphase des Defizits neutralisiert: "... the shift in the Is-curve is a once-for-all shift ... So long as the deficit continues, and continues to be financed ... the LM-curve continues to move ... Is there any doubt, that this effect must swamp the effect of the once-for-all shift of the Is-curve ?"1)

Gegen die monetaristische Meinung einer mittelfristigen Verdrängung privater Ausgaben aufgrund konventioneller Portfolio-crowding-out-Effekte sind eine Reihe von Argumenten vorgebracht worden. Hierum geht es im folgenden.

3. Würdigung

a) Zur Vermögensabhängigkeit der Geldnachfrage

Im vorliegenden Modell, in dem das Portefeuille der Privaten nur aus Zentralbankgeld und Staatspapieren besteht, und in dem sich in Folge einer Emission von Staatstiteln das Portefeuille der Privaten um diesen Betrag vergrößert, kommt es deswegen zu einem konventionellen Portfoliocrowding-out, weil die Privaten einen Teil des durch die Staatsverschuldung erfahrenen Vermögenszuwachses in Form von Kasse halten wollen. Aufgrund dieses Diversifikationswunsches entsteht auf dem Geldmarkt ein NachfrageüberschuB, und der zins auf Staatspapiere steigt solange, bis der vermögensinduzierte Wunsch, mehr Kasse zu halten, durch einen zins- und einkommensinduzierten Wunsch, weniger Kasse zu halten, kompensiert wird. Die zinserhöhung verdrängt private Investitionsausgaben.

Daraus kann man nun folgern: Wollen die Privaten nicht mehr Geld halten, wenn der Staat Forderungstitel in ihren Porte-

1) Friedman, M., Comments on therisari.6ibiser -9.70-3631-5519749. Downloaded from PubFactory at 01/11/2019 07:15:17AM 
feuilles unterbringt, entsteht auf dem Geldmarkt kein Nachfrageüberschu $B$, und die Zinsen bleiben mithin konstant", d.h. "if money demand is independent of wealth, then portfolio crowding-out cannot occur"2)

Bei einer vermögensunabhängigen Geldnachfrage ist im Privatausgabenmultiplikator $(2-2-3)$ der Koeffizient $L_{V}=0$, und der Privatausgabenmultiplikator geht in den des Grundmodells (1-4-33) über. Im IS-LM-Diagramm kommt es zu keiner Linksverschiebung der LM-Kurve, d.h. sowohl in der Entstehungsphase des Defizits als auch in der reinen Finanzierungsphase bleibt die LM-Kurve in ihrer Ausgangslage LMo (vgl. $\mathrm{Abb} .2$ und $\mathrm{Abb}, 3)$.

DaB die Privaten nicht mehr Geld nachfragen, wenn ihr vermögen zunimmt, begründen Ando und Shel13) damit, in der Realität gäbe es Vermögensanlagen, die einerseits zinserträge abwerfen und andererseits ihrer Kurzfristigkeit wegen keine nennenswerten Liquiditätsnachteile gegenüber dem Geld aufweisen. Im Portefeuille der Privaten nehmen solche Wertpapiere die der Spekulationskasse zugedachte Aufgabe wahr, und Geld wird allein als Transaktionsmittel gehalten. Plaziert der staat langfristige Forderungstitel in den Portefeuilles der Privaten, so wünschen die Vermögensanleger unter diesen Umständen den durch die Staatsverschuldung erfahrenen Vermögenszuwachs nur auf die zinstragenden Vermögensobjekte zu diversifizieren. Die Nachfrage nach zinslosen Aktiva bleibt

1) Vgl. Friedman, B., Crowding Out or Crowding In? Economic Consequences of Financing Government Deficits, a.a.0., s. $622 \mathrm{ff.}$; Currie, D.A., Monetary and Fiscal Policy and the Crowding-Out Issue, a.a.0., S. $13 \mathrm{ff}$; Butkiewicz, J.L., Outside Wealth, the Demand for Money and the Crowding out Effect, in: Journal of Monetary Economics, 1979, S. $249 \mathrm{ff}$.

2) Friedman, B., Crowding Out or Crowding In? Economic Consequences of Financing Government Deficits, a.a.o., s. $620 \mathrm{f}$.

3) Vgl. Ando, A., Shell, K., Appendix zu Ando, A., Modigliani, F., Some Reflections on Describing Structures of Financial Sectors, in: Fromm, G., Klein, L.R., (Hrsg.), The Brookings Model: Perspective and Recent Developments, Amsterdam, 1975, S. 560 ff.; für eine Kritik hierzu vgl. auch Barbosa, A.S.P., The Role of Wealth in the Money Demand Function: A Comment,

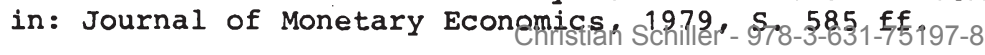


konstant.

Folgende zwei Uberlegungen sprechen allerdings gegen die These, daB es in einem Portfolio, welches ein kurzfristiges Wertpapier enthält, zu keinem GeldnachfrageüberschuB kommt, wenn der staat Forderungstitel emittiert.

Zum ersten werden nicht nur güterwirtschaftliche Transaktionen, sondern auch portfoliotransaktionen mit Hilfe von Geld abgewickelt. Weil mit steigendem Vermögen der umfang der zu bewältigenden Portfolioumschichtungen zunimmt, ist die Vermögensreagibilität der Nachfrage nach zahlungsmitteln gröBer Null.

Zweitens ist $z u$ bedenken, daB einmal für die Halter von Transaktionskasse Zinsgewinne $z u$ erzielen sind, wenn sie temporär Geld in das zinstragende,kurzfristige Aktivum umwandeln, d.h. die Transaktionskasse ist vom kurzfristigen Zins abhängig. Zum anderen sinkt im vorliegendem Modell infolge des zusätzlichen Angebots an (langfristigen) Staatstiteln der kurzfristige zins. Dadurch verliert die temporäre Umwandlung von zinsloser Kasse in zinstragende, kurzfristige Aktiva an Attraktivität. Im Zuge der Emission von Staatstiteln kommt es also auch über einen zinsstruktureffekt zu einem Geldnachfrageüberschuß.

Zudem kann mit den tberlegungen von Ando und Shell zwar plausibel gemacht werden, daB infolge einer staatlichen Kreditaufnahme die Geldnachfrage nur mäBig zunimmt, nicht aber, daB nur ein schwacher konventioneller Portfolio-crowding-out-Effekt bewirkt wird. Denn in dem erweiterten Portfoliomodell wollen die Vermögensanleger den Teil des Vermögenszuwachses, den sie bei Nichtexistenz des kurzfristigen, zinstragenden Aktivums in Form von Spekulationskasse zu halten gewünscht hätten, nun in kurzfristige, zinstragende Aktiva anlegen. Der AngebotsüberschuB auf dem Markt für Staatspapiere und damit der Anstieg des (langfristigen) Zinses wird durch die Modellerweiterung nicht berührt. Da es von den beiden zinssätzen für die Investi- 
tionsentscheidung der Privaten im wesentlichen auf den langfristigen Zins ankommt, wird das AusmaB des Portfoliocrowding-out durch eine Erweiterung des Portefeuilles der Privaten um ein kurzfristiges, zinstragendes Aktivum nicht vermindert.

\section{b) Berücksichtigung des Lerner-Effekts}

Die Hypothese, daB Veränderungen des Vermögens der Privaten ihren Konsum beeinfluBen, bezeichnet man als "Vermögen-Konsum-Relation"1) In die stabilisierungspolitische Diskussion eingebracht worden ist sie von klassischen Autoren, die sich damit gegen die Angriffe von Keynes zur Wehr setzten, wonach die Privatwirtschaft nicht automatisch zur stabilität tendiere und staatliche Interventionen deswegen unvermeidlich seien. Lerner hat diese Hypothese in einer speziellen Ausprägung dagegen zur Stützung der Keynesschen Position, insbesondere zur Begründung seiner Forderung nach einer "funktionalen Finanzpolitik", verwendet ${ }^{2}$ ! Weil er auf Vermögensvariationen abstellte, welche in einer mengenmäBigen Ausweitung des von den Privaten gehaltenen Bestandes an Staatspapieren ihre Ursache haben, werden daraus resultierende Konsumerhöhungen auch mit "LernerEffekt" etikettiert.

Um Lerners Uberlegungen in unserer Analyse zu berücksichtigen, wird das private Vermögen als Argument in die Konsumfunktion eingefügt:

1) Vgl. Monissen, H.G., Konsum und Vermögen. Analyse der Konsum-Vermögen-Relation im makroökonomischen Zusammenhang, Göttingen, 1968, s. 11 ff.; Yawitz, J., Economically Relevant Wealth: Its Specification and Role in Consumption, Diss., University Microfilms, Ann Arbor, Michigan, 1972.

2) Lerner, A.P., Functional Finance and the Federal Debt, in: Social Research, 1943, ubersetzt in: Nowotny, E. (Hrsg.), రffentliche Verschuldung, Stuttgart, New York, 1979, S. 93. 
$(2-2-4) \quad C=C(Y-T, V)$ mit $\quad C_{V}>0$.

Eine Plazierung von Staatspapieren in den Portefeuilles der Privaten zeitigt nunmehr zwei konventionelle Vermögenseffekte: Infolge des Portfolio-crowding-out werden weiterhin zinsabhängige Privatausgaben zurluckgedrängt. Weil der private Konsum vermögensabhänglg ist, werden nun aber auch die Ausgaben fur Konsumguter angeregt.

Die relative Stärke der beiden Vermögenseffekte ist in der Entstehungsphase des Defizits vergleichsweise wenig wichtig. Denn hier sind im vorliegenden Modell die beiden Vermögenseffekte lediglich zwel von insgesamt vier induzierten Privatausgabenteileffekten. Wird bei den Rechenoperationen berücksichtigt, daB auch der private Konsum vermögensabhängig ist, verändert sich in der Entstehungsphase des Defizits das Niveau der Privatausgaben nach MaBgabe des folgenden Multiplikators:

$$
(2-2-5) \frac{\mathrm{d}(\mathrm{C}+\mathrm{I})}{\mathrm{d} \overline{\mathrm{G}}}=\frac{1+\mathrm{C}_{V}-\frac{\mathrm{I}_{i}}{\bar{L}_{i}} \mathrm{~L}_{V}}{1-\mathrm{C}_{Y}\left(1-\mathrm{T}_{\mathrm{Y}}\right)+\mathrm{C}_{V} \cdot \mathrm{T}_{Y}+\frac{\mathrm{I}_{i}}{\mathrm{~L}_{i}}\left(\mathrm{~L}_{\mathrm{Y}}-\mathrm{L}_{V} \cdot \mathrm{T}_{Y}\right)}-1
$$

Im Vergleich zum Privatausgabenmultiplikator bei

lediglich vermögensabhängiger Kassenhaltung (2-2-3) taucht im Zähler und im Nenner jeweils ein neuer Term auf. Davon kommt es vor allem auf den Im zähler an: Damit in der Entstehungsphase des Defizits durch die Vermögenserhöhung private Ausgaben zurückgedrängt werden, reicht es nun nicht mehr aus, wenn der Term $I_{i} / L_{i} \cdot I_{S}$ einen positiven Hert annimmt; er muB vielmehr einen gröBeren wert als $C_{V}$ haben.

Graphisch (vgl. Abb. 4) kommt der Lerner-Effekt in einer weiteren Rechtsverschiebung der IS-Funktion zum 
Abbildung 4 : Lerner-Effekt und konventioneller Portfoliocrowding-out-Effekt in der Entstehungsphase des Defizits

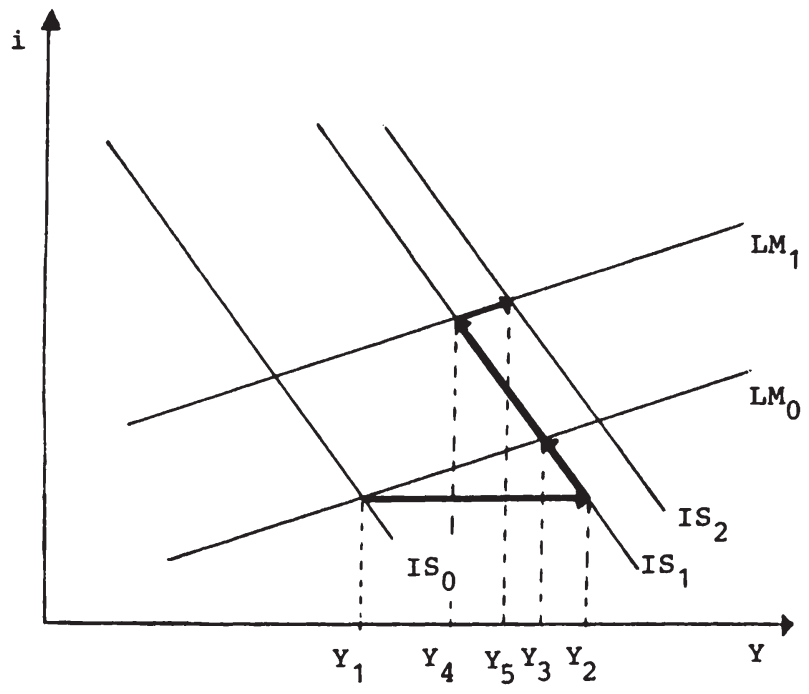

Ausdruck $\left(I S_{1} \longrightarrow I S_{2}\right)$.

GroBe Bedeutung hat der Lerner-Effekt dagegen in der reinen Finanzierungsphase des Defizits ${ }^{1}$ ! Denn jetzt entscheiden über die Richtung, in welche sich das Privatausgabenniveau entwickelt, allein die beiden Vermögenseffekte. Nur wenn der Vermögen-Konsum-Impuls von dem Vermögen-Geldnacherage-Impuls überkompensiert wird, verringert sich von Haushaltsperiode zu Haushaltsperiode das Niveau der privaten Ausgaben; nur wenn

1) Vgl. Blinder, A.S., Solow, R.M., Does Fiscal Policy Matter?, a.a.o., S. 321 ff.; Infante, E.F., Stein, J.L., Does Fiscal Policy Matter?, in: Journal of Monetary Economics, 1976, S. 481 ff.: Burrows, P. , The Government Budget Contraint and the MonetaristKeynesian-Debate, a.a.0., S. $77 \mathrm{ff}$. 
Abbildung 5: Lerner-Effekt und konventioneller Portfoliocrowding-out-Effekt in der reinen Finanzierungsphase des Defizits

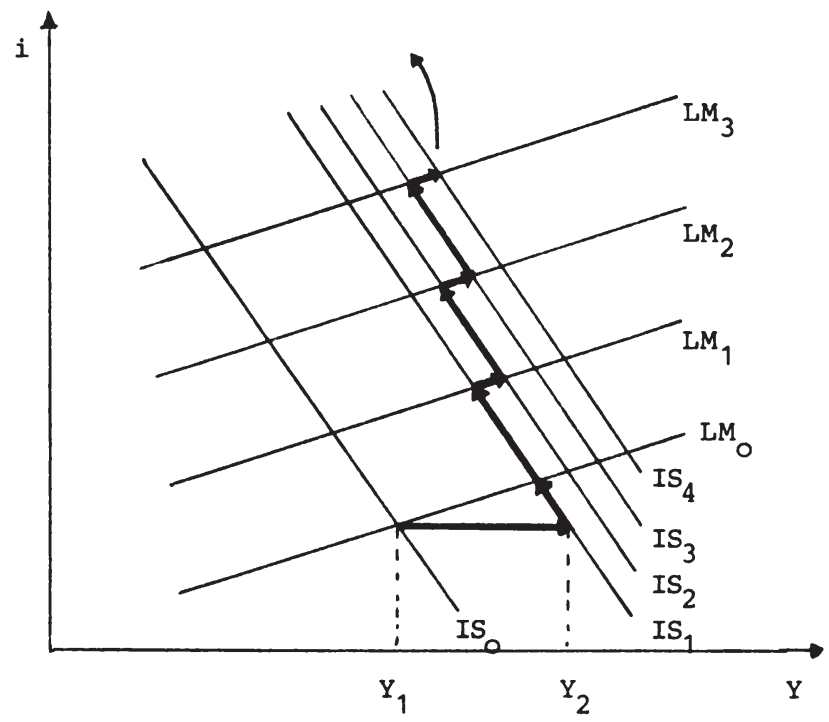

also

(2-2-6) $\quad C_{V}<\frac{I_{i}}{L_{i}} L_{V}$

ist (graphisch: wenn in den einzelnen Haushaltsperioden der reinen Finanzierungsphase des Defizits die Privatausgaben durch die Linksverschiebung der LM-Funktion um mehr abnehmen als durch die Rechtsverschiebung der ISFunktion zunehmen), sinkt von Haushaltsperiode zu Haushaltsperiode die private Güternachfrage (vgl. Abb. 5). 
Andernfalls steigt in der reinen Finanzierungsphase des Defizits das Niveau der Privatausgaben, weil in jeder Haushaltsperiode via Lerner-Effekt mehr Privatausgaben angeregt als durch den Portfolio-crowding-out-Effekt verdrängt werden.

Die vorliegenden empirischen Untersuchungen weisen auf einen signifikanten EinfluB des privaten Vermögens auf die private Konsumnachfrage hin. Einem von Patinkin ${ }^{1)}$ zusammengestellten Uberblick kann man entnehmen, daB der private Konsum etwa von 4 bis 8 vom Hundert steigt, wenn sich das private vermögen erhöht. Mithin ist auf jeden Fall eine Anregung des privaten Konsums in Rechnung zu stellen, wenn man den Privatausgabeneffekt einer plazierung von Staatspapieren in den Portefeuilles der Privaten ermittelt.

Dies bedeutet zum einen, daB sich als Folge der beiden konventionellen Vermögenseffekte in der Entstehungsphase und in der reinen Finanzierungsphase des Defizits die Struktur der Privatausgaben zugunsten des Konsums verändert. Zum anderen ist der Lerner-Effekt ein Argument gegen die monetaristische These einer ständigen Verminderung des Niveaus der privaten Ausgaben in der reinen Finanzierungsphase des Defizits.

1) Vgl. Patinkin, D., Money, Interest, and Prices, New York, $1965^{2}$, S. $651 \mathrm{ff}$. 
c) Kontraktive Vermögenseffekte und langfristige Stabilität

Die monetaristische These, in der reinen Finanzierungsphase des Defizits wirden infolge von privatausgabenrestringlerenden Vermögenseffekten anfängliche Expansionstendenzen konterkariert, provozierte eine Diskussion, in welcher der Nachweis gefuhrt wurde, daB privatausgabenrestringierende Vermögenseffekte und langfristige stabilităt des rkonomischen Systems nicht miteinander vereinbar sind. Anders gewendet: Das bkonomische System 1st stabil, wenn es nach einem exogenen Schock, z.B. In Form einer dauerhaften Ausdehnung der staatlichen Güternachfrage, $z u$ einem Gleichgewicht konvergiert; dabei müssen aber die konventionellen Vermögenseffekte die privaten Ausgaben in summa stimulieren und dürfen sie nicht verdrängen ${ }^{1)}$ ?

Im vorliegenden Modell nehmen, weil Vermögenseffekte berücksichtigt werden, die Gesamt- und die Privatausgaben erst dann ihren endgültigen Wert an, wenn sich neben einem stromgleichgewicht, d.h. der Kompatibilität der geplanten Finanzierungssalden, auch ein Bestandsgleichgewicht eingestellt hat. Allgemein ist ein Bestandsgleichgewicht dadurch charakterisiert, daB die in Betracht gezogenen Bestände konstant bleiben, hier: die Zentralbankgeldmenge und der Bestand an Staatsschuldtiteln bel den Privaten. Damit eine langfristige Ruhelage einkehrt, müssen deshalb nicht nur die Gleichgewichtsbedingungen für den Güter- und den Geldmarkt erfullt sein, sondern es muB auch der offentliche Haushalt ausgeglichen sein:

1) Für einen tberblick vgl. Carlson, M.K., Spencer, R.W., Crowding-Out and its Critics, a.a.o., S. 11 ff.; Choudhry, N.N., Integration of Fiscal and Monetary Sectors in Econometric Models: A Survey of Theoretical Issues and $\mathrm{Findings,} \mathrm{in:} \mathrm{International}$ Monetary Fund, Staff Papers, 1976, S. 396 ff.; Siebke, J., Knoll, D., Schmidberger, W.-D., Theoretische Grundlagen des crowding out Effektes, a.a.o., s. $231 \mathrm{ff}$. 
$(2-7-7) \quad \bar{G}=T(Y)$.

Ein Haushaltsausgleich macht Steuermehreinnahmen notwendig, wenn die Staatsausgaben auf Dauer erhöht werden. Um langfristig wieder einen Gleichgewichtszustand zu erreichen, muB deshalb das Einkommen hinreichend stark steigen. Dies impliziert positive langfristige Gesamt- und Privatausgabenmultiplikatoren. sie erhält man, indem Gleichung (2-2-7) total differenziert

$(2-2-8) \quad d \vec{G}=T_{Y} \cdot d Y$

und dann umgeformt wird:

$$
\begin{aligned}
& (2-2-9) \quad \frac{d Y}{d \bar{G}}=\frac{1}{T_{Y}}, \\
& (2-2-10) \quad \frac{d(C+I)}{d \bar{G}}=\frac{1}{T_{Y}}-1 .
\end{aligned}
$$

Tendiert das System zu einem Gleichgewicht und dehnt der Staat dauerhaft seine Ausgaben aus, erhöhen sich demnach die Gesamt- und die Privatausgaben auf lange Frist nach MaBgabe des reziproken Wertes des marginalen Steuersatzes; dies gilt unabhängig von der Art der staatsausgabenfinanzierung ${ }^{1)}$.

Blinder und Solow ${ }^{2}$ ) haben dieses Ergebnis aufgegriffen und als Argument gegen die Hypothese kontraproduktiver konventioneller Vermögenseffekte verwen-

1) Dieses Ergebnis wurde erstmals abgeleitet von Ott, D.J., Ott, A.F., Budget Balance and Equilibrium Income, a.a.0., s. $71 \mathrm{ff}$.

2) Vgl. Blinder, A.S., Solow, R.M., Does Fiscal Policy Matter?, a.a.O., S. $324 \mathrm{ff.;}$ Infante, E.F., stein, J.L., Does Fiscal Policy Matter?, a.a.O., s. 481 ff. 
det. Die der Gleichgewichtsbedingung (2-2-7) implizite zugrundeliegende Annahme, der staat erhebe neben einer einkommensabhängigen Steuer in jeder Haushaltsperiode eine weitere steuer in genau der Höhe der zu leistenden Zinsausgaben, haben sie durch die Annahme ersetzt, der staat finanziere die zinszahlungen aus dem einkommensabhängigen steueraufkommen. In der Blinder/ Solow-Version der staatlichen Finanzierungsrestriktion erscheinen deswegen die Zinszahlungen des Staates $\left(\mathrm{Y}^{\mathrm{B}}\right)$ als Ausgabenkomponente; zudem werden sie als Teil der steuerbemessungsgrundlage berücksichtigt:

$$
(2-2-11) \quad \bar{G}+Y^{B}=T\left(Y+Y^{B}\right)+\left(B-\bar{B}_{-1}\right) \text {. }
$$

Eine langfristige Ruhelage erfordert einen Haushaltsausgleich; und wird, zur Vereinfachung, $T_{Y}=T_{Y B}=t$ gesetzt, tritt

$(2-2-12) \quad \bar{G}+Y^{B}=t \cdot\left(Y+Y^{B}\right)$

an die Stelle von Gleichung (2-2-7). Durch totale Differenzierung

$$
\text { (2-2-13) } d \bar{G}+d Y^{B}=t \cdot d Y+t \cdot d Y^{B}+\underbrace{Y^{B} \cdot d t+Y \cdot d t}_{=0}
$$

und Umformen erhält man die langfristigen Ausgabenmultiplikatoren:

$$
\begin{aligned}
& (2-2-14) \frac{d Y}{d \bar{G}}=\frac{1+(1-t) \cdot \frac{d Y^{B}}{d \bar{G}}}{t} \\
& (2-2-15) \frac{d(C+I)}{d \bar{G}}=\frac{(1-t) \cdot\left(1+\frac{d Y^{B}}{d \bar{G}}\right)}{t} .
\end{aligned}
$$


Im Vergleich $\mathrm{zu}$ den Ausgabenmultiplikatoren (2-2-9) und $(2-2-10)$ haben sie einen groberen Wert; dies rührt daher, daB jetzt nicht nur die Transformationsausgaben, sondern auch die zinszahlungen aus dem einkommensabhängigen Steueraufkommen $z u$ decken sind. Mithin muB die Einkommenserhöhung vergleichsweise stärker ausfallen, um das Haushaltsdefizit abzubauen ${ }^{1 !}$ ?

Aus alledem folgt: Damit die Privat- und die Gesamtausgaben wieder eine endgültige Ruhelage einnehmen, darf sich der Bestand an Staatsschuldtiteln in Händen der Privaten nicht mehr verändern. Die auf Dauer erhöhten staatsausgaben werden aber nur dann in voller Höhe durch Steuermehreinnahmen gedeckt, wenn deren Bemessungsgrundlage hinreichend gestiegen ist. Ein neues langfristiges Bestandsgleichgewicht bedingt mithin positive Gesamt- und Privatausgabenmultiplikatoren. Lerner-Effekt und konventioneller Portfolio-crowding-out-Effekt muissen deshalb per Saldo die Privatausgaben erhöhen.

Mindern sie dagegen insgesamt die Privatausgaben, d.h. "if the monetarists are right, the system is unstable"2). Die Gesamtausgaben verringern sich dann in der reinen Finanzierungsphase ständig und mit zunehmendem Tempo, ohne daB eine neue Ruhelage in sicht ist. Kontraktive Vermögenseffekte und langfristige stabilität sind folglich nicht miteinander kompatibel. Aus diesem

1) Weil bei einer Zentralbankfinanzlerung $\mathrm{dY}^{\mathrm{B}} / \mathrm{d} \overline{\mathrm{G}}=0$ ist, ist der langfristige Gesamtausgabenmultiplikator einer durch Notenbankkredit finanzierten Ausdehnung der staatlichen Güternachfrage gleich $1 / t$ und der langfristige Privatausgabenmultiplikator $(1-t) / t$ : "contrary to the usual supposition, the long-run multiplier for bond-financed deficit spending exceeds that for money-financed deficit spending". Blinder, A.S., Solow, R.M., Does Fiscal Policy Matter?, a.a.0., S. 327.

2) Blinder, A.S., Solow, R.M., Does Fiscal Policy Matter?, a.a.0., S. 336 . 
Grund verwerfen Blinder und solow mit anderen "jene monetaristische Position, die einen vollständigen crowding-outEffekt unterstellt, well er innerhalb des erweiterten ISLM-Paradigmas die Instabilität des systems impliziert. Diese Implikation aber ist konträr zur axiomatischen Ausgangsthese der Monetaristen, nach der das kapitalistische Wirtschaftssystem stabil in dem Sinne ist, daß es exogene schocks absorbiert"1). Für unsere Fragestellung hat dieser Einwand allerdings wenig Gewicht. Denn zum einen ist er mehr methodologischer Natur und zum anderen hauptsächlich auf lange Sicht von Bedeutung. Zudem gibt es neben der stabilitätsthese noch eine andere, eher pragmatische Ausgangsthese der Monetaristen, nämlich die Unsicherheit über die kurzfristigen Wirkungen stabilisierungspolitischer Maßnahmen.

d) $\mathrm{Zu}$ den Substitutionsbeziehungen zwischen den Vermögenskomponenten

aa) Grundlage: ein erweitertes makroökonomisches Portfoliomodell

Das makroökonomische Portefeuille hat in unserer Betrachtung (von der Erörterung der Ando/Shell-These abgesehen) bislang nur zwei Vermögensgüter enthalten: (zinsloses) Zentralbankgeld und (zinstragende) Staatspapiere. Diese enge Vermögensdefinition wird häufig verwendet, und die Vorgehensweise wird damit begründet, alle zinstragenden Vermögensobjekte seien gute Substitute: Dann entwickeln sich deren Effektivverzinsungen ziemlich ähnlich, und es genügt, sich auf ein repräsentatives zinstragendes Vermögensgut zu konzentrieren. Löst man, Tobin folgend, diese Annahme auf, disaggregiert man also die zinstragenden vermögensobjekte und zieht unterschiedliche substitutionsbeziehungen zwischen den Vermögensgütern ins Kalkül, ist

1) Siebke, J., Knoll, D., Schmidberger, K.-D., Theoretische Grundlagen des crowding out Effektes, a.a.0., S. 235; vgl. auch Scarth, W.M., A Note on the "Crowding Out" of Private Expenditures by BondFinanced Increases in Government Spending, in: Journal of Public Economics, Nr. 5, 1976, S. $385 \mathrm{ff}$. 
ein Portfolio-crowding-out nicht mehr eine Modellimplikation, sondern eines (wenn auch das wahrscheinlichere) von zwei denkbaren Ergebnissen einer Plazierung von Staatspapieren im privaten Bereich ${ }^{1 !}$.

Die nun verwendete Vermögensgleichung enthält neben dem Zentralbankgeld (MZ) und den Staatsschuldtiteln (B) auch das bereits existierende Realkapital (K):

$(2-2-16) \quad V=M Z+B+K$.

Zu dem Geldmarkt und dem Markt für Staatspapiere tritt ein weiterer Bestandsmarkt hinzu, auf dem bereits produziertes Realkapitel gehandelt wird, entweder indirekt in Form verbriefter Ansprüche, wie z.B. Aktien, oder direkt, wie z.B. im Fall von Immobilien.

Wird der sich dort bildende Gleichgewichtspreis $\left(P^{K}\right)$ mit den Nominalzinsen des Realkapitals $\left(E^{K}\right)$ in Beziehung gebracht, erhält man die Effektivverzinsung des existierenden Realkapitals (ik). Diese Rendite, die Tobin mit "Angebotspreis des Kapitals" bezeichnet, gibt an, zu welcher effektiven Ertragsrate die Vermögensbesitzer bereit sind, den bestehenden Sachkapitalbestand in ihren Portefeuilles zu halten. Ihre Höhe, wie auch die der

1) Die makroökonomische Portfoliotheorie ist in mehreren Beiträgen von vor allem Tobin entwickelt worden. Vgl. hauptsächlich Tobin, J., An Essay on the Principles of Debt Management, in: Fiscal and Debt Management Policies, 1963, übersetzt: Grundsätze der Geld- und Staatsschuldenpolitik, Baden-Baden, 1978; derselbe, The Equilibrium Approach to Monetary Theory, in: Journal of Money, Credit, and Banking, 1969, S. 15 ff., übersetzt: Ein allgemeiner Gleichgewichtsansatz zur Geldtheorie, in: Brunner, K., Monissen, H.-G., Neumann, M.J.M. (Hrsg.), Geldtheorie, Köln, 1974, S. $219 \mathrm{ff.;}$ für einen Uberblick vgl. Kath, D., Euba, N., Die makroökonomische Portfoliotheorie, in: Wirtschaftswissenschaftliches Studium, 1975, S. $458 \mathrm{ff}$. 
Effektivverzinsung der Staatsschuldtitel, ist das Ergebnis der Anpassung der gewünschten Struktur des Portefeuilles an die exogen gegebene, tatsächliche Portefeuillestruktur. Dabei lassen sich, wie gesagt, die Wirtschaftssubjekte von Risiko- und Ertragsgesichtspunkten leiten.

Unter Ertragsgesichtspunkten betrachtet sind alle Vermögensgüter gute Substitute. Das Ertragskalkül impliziert demnach eine gleichgerichtete Reaktion der Nachfrage nach einem Vermögensgut auf die Veränderung der eigenen Verzinsung und eine gegenläufige auf die Veränderung der Rendite von konkurrierenden Vermögensobjekten. Wenn allein die Renditen für die Portefeuillestruktur maBgebend wären, würden die Vermögensbesitzer immer nur ein Vermögensgut $z u$ halten wünschen und zwar dasjenige, welches für die Planungsperiode den höchsten Ertrag verspricht.

Risikoüberlegungen sorgen dafür, daß das Vermögen auf verschiedene Komponenten aufgeteilt wird. Denn durch eine Diversifizierung läßt sich das Gesamtrisiko (des Portefeuilles) kleiner als die Summe der Einzelrisiken (der verschiedenen Vermögensgüter) machen. Aus dem Risikokalkül resultiert folglich ein komplementäres Verhältnis zwischen Vermögensgütern, welche mit voneinander unabhängigen Risiken behaftet sind. Dies sind gemäB Tobin ${ }^{1)}$ alle finanziellen Vermögensgüter auf der einen Seite - hier: Zentralbankgeld und Staatsschuldtitel - und alle sachlichen Vermögensgüter auf der anderen Seite - hier: das in den Vorperioden produzierte Realkapital.

1) Vgl. Tobin, J., Grundsätze der Geld- und Staatsschuldenpolitik, a.a.O., S. 50 ff.; vgl. hierzu auch Ketterer, K.-H., Karmann, A., Einige theoretische und empirische Aspekte der Zinswirkungen der Staatsverschuldung, in: Karmann, A. (Mitverf.), Uber Zins, Geldwert, Versicherungen und "kapitalistischer Geist", Königstein/Ts., 1981, S. $11 \mathrm{ff.;}$ Franke, H.-H., Portfolioeffekte öffentlicher Kreditnahme, Berlin 1981, S. $48 \mathrm{ff}$. und Bookstaber, R., The Effect of Inflation Uncertainty on "Crowding-Out", in: Journal of Macroeconomics, 1980, S. $85 \mathrm{ff}$. 
Finanzielle Vermögenstitel unterliegen hauptsăchlich folgenden zwei Risiken: Zum einen besteht die Möglichke1t, daB durch nicht korrekt antiziplerte Preissteigerungen der Realwert einer Finanzanlage unerwartet geschmälert wird. Zum anderen sorgen Kursschwankungen im Zeitablauf dafür, daB bei einem Verkauf elnes Vermögenstitels vor dessen Fälligkeit der Verkaufskurs unter dem Nominalkurs liegen kann. Demgegenüber besteht das Risiko des Haltens von Realkapital darin, daB der Sachkapitalbestand aufgrund des technischen Fortschritts (technologisches Risikol, aufgrund konjunkturell bedingter Schwankungen der gesamtwirtschaftlichen Güternachfrage (Konjunkturrisiko) und struktureller Nachfrageverschiebungen (Branchenrisiko) unerwartet rasch an wert verlieren kann.

Die Risiko- und Ertragsüberlegungen, welche die Vermögensanleger anstellen, schlagen sich im betrachteten makroökonomischen Portfoliomodell in den Werten der partiellen Kreuzableitungen nach dem $\mathrm{zinssatz}$ und dem Vermögen nieder. Bei guten Substituten sind die Kreuzableitungen nach dem $\mathrm{Z}$ ins betragsmäßig groß, nach dem Vermögen klein; bei schlechten sind die Kreuzableitungen nach dem zins betragsmäBig klein und nach dem Vermögen groB.

Das vorliegende makroökonomische Portfoliomodell wird durch sieben Gleichgewichtsbedingungen, Verhaltensgleichungen und Definitionen beschrieben (vgl. Ubersicht 6). Es ist gegenüber dem im Grundmodell als Submodell enthaltenen, rudimentären makroökonomischen Portfoliomodell drelfach erweitert:

Erstens: In Betracht gezogen werden neben dem Geldmarkt $(\mathrm{L}=$ Geldnachfrage, $\overline{\mathrm{MZ}} \mathrm{S}=$ zentralbankgeldangebot, $\mathrm{MZ}=$ tatsächliche Zentralbankgeldmenge) und dem bisher implizit berücksichtigten Markt für staatspapiere ${ } \mathrm{B}^{\mathrm{d}}=$ Nachfrage nach Staatsschuldtiteln, $\bar{B} \mathbf{S}=$ Angebot an Staatsschuldtiteln, B = tatsächlicher Bestand an Staats- 
schuldtiteln bel den Privaten) auch der Markt für bestehendes Realkapital $\left(\mathrm{K}^{\mathrm{d}}=\right.$ Nachfrage nach bestehendem Realkapital, $\overline{\mathrm{K}}^{\mathrm{S}}=$ Angebot an bestehendem Realkapital, $K$ = tatsăchlicher Bestand an Realkapitall. Zweitens: Das makrobkonomische Portfolio enthalt nunmehr zwel zinstragende Vermögensgüter; folglich gibt es auch zwei zinssätze: für Staatsschuldtitel (ib) und für bestehendes Realkapital (ik). Drittens: Wie bisher im Rahmen der Erörterung von Vermögenseffekten praktizlert, sind die Bestandsnachfragen vom Vermögen (V) abhängig.

Ubersicht 6: Das erweiterte makroökonomische Portfoliomodell in seiner strukturellen Form

$$
\begin{aligned}
& (2-2-17) \quad L(i b, i k, V)=\overline{M Z} \\
& \text { mit } L_{i b}, L_{i k}<0 ; L_{v}>0 \text {, } \\
& (1-4-24) \quad M Z=L=\overline{M Z} S \\
& (2-2-18) \quad B^{d}(i b, i k, v)=\bar{B}^{s} \\
& \text { mit } B_{i b}^{d}, B_{V}^{d}>0 ; B_{i k}^{d}<0 \text {, } \\
& (2-2-19) \quad B=B^{d}=\bar{B}^{S} \\
& (2-2-20) \quad k^{d}(1 b, i k, v)=\bar{K}^{s} \\
& \text { mit } k_{i k}^{d}, k_{V}^{d}>0 ; k_{i b}^{d}<0 \text {, } \\
& (2-2-21) \quad K=K^{d}=\bar{K}^{s} \\
& (2-2-16) \quad V=M Z+B+K
\end{aligned}
$$


bb) Makroökonomisches Portfolio und private Ausgaben

Die Anbindung des Gütermarktes an das makroökonomische Portfolio erfolgt - wie im Grundmodell - über die Investitionsfunktion. Allerdings ist diese hier anders spezifiziert. Ob neues Realkapital gebildet wird, hängt in der Tobinschen Formulierung ${ }^{1)}$ von dem Verhältnis zwischen der erwarteten Rendite auf neu produzierte Einheiten des Realkapitals und dem Angebotspreis des Kapitals (ik) ab. Ist dieses größer Eins, wird Investitionsgüternachfrage entfaltet. Bei konstanter erwarteter Rendite auf neu produzierte Einheiten des Realkapitals sind die Nachfrage nach Investitionsgütern und der Angebotspreis des Kapitals invers miteinander verknüpft:

$(2-2-22) \quad I=I(i k)$ mit $I_{i k}<0$.

Anders, unter Verwendung des Tobinschen $q=\frac{E^{I} / P^{I}}{E^{K} / P^{K}}$ formuliert: Bei gleichen Erträgen von bereits bestehenden und noch zu produzierenden Kapitalgütern ( $\mathrm{E}^{\mathrm{K}}=\mathrm{E}^{\mathrm{I}}$ ) wird immer dann investiert, wenn die Wiederbeschaffungskosten ( $\mathrm{P}^{\mathrm{I}}$ ) des Sachkapitals geringer sind als sein Marktwert $\left(\mathrm{P}^{\mathrm{K}}\right)$, wenn also q kleiner Eins ist.

1) Vgl. hierzu auch Ketterer, K.-H., Vollmer, R., Bestimmungsgründe des Realzinsniveaus. Eine empirische Untersuchung für die Bundesrepublik Deutschland, in: Ehrlicher, W. (Hrsg.), Geldpolitik, Zins und Staatsverschuldung, Schriften des Vereins für Socialpolitik, Band 111, Berlin, 1981, s. 58 ff. 
cc) Portfolio-crowding-out oder Portfolio-crowding-in?

In welche Richtung sich das Tobinsche $q$ im Zuge einer Plazierung von Staatspapieren im privaten Bereich entwickelt, kann im vorliegenden Modell ${ }^{1)}$ am zinsmultiplikator dik/dB abgelesen werden. Bei der Ableitung des Zinsmultiplikators kann man wegen der Vermögensrestriktion einen Markt - hier: den für bestehendes Realkapital - streichen. Denn auf diesem stimmen Angebot und Nachfrage überein, wenn sich die beiden anderen Märkte im Gleichgewicht befinden.

Wir bilden das totale Differential; das entstehende Gleichungssystem lautet unter Berücksichtigung von $d V=d B$ und $d M z=0$ in Matrixschreibweise:

$(2-2-23)$

$$
\left[\begin{array}{cc}
L_{i b} & L_{i k} \\
B_{i b}^{d} & B_{i k}^{d}
\end{array}\right] \cdot\left[\begin{array}{l}
d i b \\
d i k
\end{array}\right]=\left[\begin{array}{l}
-L_{V} \cdot d B \\
\left(1-B_{v}^{d}\right) \cdot d B
\end{array}\right] \text {. }
$$

1) Für ähnliche Wirkungsanalysen vgl. Korliras, P.G., Bond-Financed Deficits and the Crowding-Out Hypothesis, Working Paper Nr. 37, University of Pittsburgh, 1976, S. $11 \mathrm{ff}$; Siebke, J., Der "crowding-out"-effect in einem Portfolio-Makromodell, in: Albach, H., u.a. (Hrsg.), Quantitative Wirtschaftsforschung. Wilhelm Krelle zum 60. Geburtstag, Tübingen, 1977, S. 657 ff.; Friedman, B. . Crowding Out or Crowding In? Economic Consequences of Financing Government Deficits, a.a.0., S. $608 \mathrm{ff.;}$ Karmann, A., Ketterer, K.-H., Staatsverschuldung und Investitionstätigkeit: Ist ein "Crowding out" die zwangsläufige Folge einer Zunahme der staatsverschuldung?, in: Göppl, H., Henn, R. (Hrsg.), Geld, Banken und Versicherungen, Beiträge zum 1. Symposium Geld, Banken und Versicherungen an der Universität Karlsruhe vom 11.-13. Dezember 1980, Königstein/Ts., 1981, S. $230 \mathrm{ff}$. und Franke, H.-H., Portfolioeffekte öffentlicher Kreditnahme, a.a.0., S. $25 \mathrm{ff}$. 
Nach der Cramer-Regel und bei Beachtung von "adding-up-Bedingungen" ${ }^{1)}$ ergibt sich als Zinsmultiplikator:

$$
(2-2-24) \frac{d i k}{d \bar{B}}=\frac{-L_{B} \cdot K_{1 b}^{d}+L_{1 b} \cdot K_{B}^{d}}{L_{i b} \cdot B_{1 k}^{d}-L_{1 k} \cdot B_{1 b}^{d}} \geqslant 0
$$

Wegen $L_{1 b}, B_{1 k}^{d}, L_{1 k}<0$ und $B_{1 b}^{d}>0$ hat der Nenner stets einen positiven wert. Wegen $L_{B^{\prime}} K_{B}^{d}>0$ und $K_{1 b^{\prime}}^{d} L_{i b}<0$ kann der zähler und damit auch der Bruch einen positiven oder einen negativen Wert haben. Das Ergebnis hängt vom Wert der partiellen Ableitungen im zähler und damit von den Substitutionsbeziehungen zwischen den Vermögensgütern $a b$.

Nach Tobin sind Staatsschuldtitel und Zentralbankgeld gute, Staatsschuldtitel und Realkapital schlechte Substitute. Er würde deshalb den Zinsmultiplikator (2-2-22) folgendermaBen interpretieren: Tritt der staat mit einem zusätzlichen Angebot an den Markt für Staatspapiere, steigt dort der Zins, was konkurrierenden Vermögensanlagen Attraktivität nimmt. Weil zentralbankgeld und Staatsschuldtitel bereitwillig gegeneinander ausgetauscht werden, reagieren darauf die Vermögensbesitzer mit einem vergleichsweise starken Abzug von Mitteln vom Geldmarkt, d.h. $\mathrm{L}_{1 \mathrm{~b}}$ ist groB. Auf den Markt für bestehendes Realkapital pflanzt sich die Erhöhung des Effektivinses von staatsschuldtiteln deswegen nur unwesentlich fort; es ist mithin $\mathrm{k}_{1 \mathrm{~b}}^{\mathrm{d}} \mathrm{klein}$.

1) "Adding-up-Bedingungen" besagen, daB die Summe der Nachfrageänderungen nach den einzelnen Vermögenskomponenten bei konstantem Gesamtvermögen gleich Null ist und bei einer Veränderung des Vermögens gerade dieser entspricht. 
Dieser, die Investitionsnachfrage dämpfende EinfluB hält sich also in Grenzen und wird dadurch kompensiert, daB die Privaten ihr zusätzliches Vermögen, was sich ausschlieblich in einem erhohten staatspapierbestand manifestiert, als unbefrledigend empfinden und deshalb vermehrt Vermogensobjekte mit andersartigen Risiken, sprich: Realkapital, nachfragen. Mithin ist $k_{B}^{d}$ groB und $L_{B}$ klein. Im Ergebnis weist deshalb $d 1 k / d B$ einen negativen Wert auf. Die Vermoggensbesitzer sind jetzt mit einem vergleichsweise geringen Angebotspreis des Kapitals zufrieden. Dadurch wird die Nachfrage nach neu zu produzierendem Sachkapital stimuliert. Die Staatsverschuldung hat kein Portfollo-crowding-out, sondern ein Portfoliocrowding-in zur Folge.

Dieses Resultat steht und fält mit der Hypothese, Staatspapiere und Realkapital seien schlechte Substitute. Trifft das Gegenteil $\mathrm{zu}$, wie bisher angenommen, dann reagieren die Vermögensbesitzer auf eine Erhöhung des Zinssatzes für Staatspapiere mit einer Reallokation des Portefeuilles in die umgekehrte Richtung: Es wird versucht, Realkapital abzustoBen, um Staatspapiere zu kaufen. Es ist $\mathrm{K}_{i b}^{\mathrm{d}}$ groB und $\mathrm{L}_{i b}$ klein. Die Erhöhung der zinstragenden Vermögensgüter in ihren Portefeuilles wird zum AnlaB genommen, auch Zentralbankgeld aufzustocken. Demnach ist $L_{B}$ groB und $K_{B}^{d}$ klein. Der negative Term im zahler verliert und der positive gewinnt an Gewicht. Bei hinreichenden Werten wird also $\mathrm{dik} / \mathrm{dB}>0$. Dies 1st die monetaristische These; sie ist verknüpft mit der Annahme, die zinstragenden Aktiva seien gute Substitute"

1) Die hier angestellten Erwägungen werden mit denen des vorangegangenen Abschnitts verknüpft von Cohen, D. , McMenamin, J.S., The Role of Fiscal Policy in a Financially Disaggregated Macroeconomic Model, in: Journal of Money, Credit, and Banking, 1978, S. 322 ff. 
Sind nun Staatspapiere gute oder schlechte Substitute? Die Antwort darauf hängt, wie gesagt, davon $a b$, welche Rolle Risikoüberlegungen bei der Strukturierung des Portefeuilles spielen. Haben sie viel Gewicht, wovon Keynesianer ausgehen, sind Staatspapiere und Realkapital schlechte Substitute; haben sie nur eine geringe Bedeutung, was Monetaristen annehmen, werden Staatspapiere und Realkapital dagegen ziemlich bereitwillig gegeneinander ausgetauscht.

Risikoerwägungen treten in den Hintergrund, wenn (erstens) die Risikoaversion bei den Vermögensanlegern gering ist. "Risk-lovers" achten bei der Aufteilung ihres Vermögens hauptsächlich auf die Renditen und ziehen nur wenig ins Kalkül, welche Risiken sie eingehen, wenn sie ein Vermögensobjekt erwerben. Bei einer Zunahme des Bestandes an Staatspapieren in ihren Portefeuilles entsteht mithin kaum der Wunsch, auch mehr Realkapital zu halten, und auf einen Anstieg des $\mathrm{Z}$ inses auf Staatspapiere reagieren sie mit einer massiven Einschränkung der nun unlukrativ gewordenen Anlage des Vermögens in Realkapital.

Neben der (subjektiven) Risikoneigung ist zum zweiten wichtig, für wie sicher die Privaten ihre Erwartungen halten. Bei vergleichsweise sicheren Erwartungen schätzen die Privaten die Möglichkeit, von nicht antizipierten Ereignissen überrascht $\mathrm{zu}$ werden, als ziemlich gering ein. Dann haben auch aus "objektiven" Gründen Risikoerwägungen keine allzu groBe Bedeutung bei der strukturierung des Portefeuilles. Demzufolge sind die Substitutionsbeziehungen zwischen Realkapital und Staatspapieren in Phasen einer konstanten wirtschaftlichen Entwicklung enger als in Phasen einer unstetigen wirtschaftlichen Entwicklung, und eine auf stetigkeit ausgerichtete Wirtschaftspolitik fördert die Bereitschaft der Privaten, Realkapital und staatspapiere gegeneinander auszutauschen. 
Ergebnis: Tobins Uberlegungen machen deutlich, daB eine Erhöhung des Zinses auf Staatspapiere nur ein grober Anhaltspunkt für das AusmaB des konventionellen Portfoliocrowding-out-Effekt ist. Denn zum einen ist für die Entscheidung der Privaten zu investieren der Angebotspreis des Kapitals wichtig. Zum anderen sorgen Risikoerwägungen bei der Strukturierung des Portefeuilles dafür, daB infolge einer staatlichen Kreditaufnahme das Renditedifferential zwischen Staatspapieren und Realkapital zunimmt, der Angebotspreis des Kapitals also weniger steigt loder unter Tobins Annahmen sogar sinkt), als der zins auf Staatpapiere. Es wird also das AusmaB des konventionellen Portfollo-crowding-out-Effekt überzeichnet, wenn man zu dessen Abschätzung als Indikator den zins auf staatspapiere verwendet.

\section{e) Tax discounting}

Von Barro ${ }^{1)}$ u.a. wird, auf Ricardo rekurrierend, die These vertreten, staatliche Anleihen würden gar nicht zum Vermögen der Privaten gehören. Demnach würden auch keine konventionellen Vermögenseffekte induziert. Denn die Privaten müssen die Mittel, die zur Verzinsung und Tilgung der von ihnen gehaltenen staatstitel notwendig sind, selbst durch Zahlung von Steuern aufbringen. Deshalb dürfen nicht nur die Staatstitel (B) als Aktivum, sondern muB auch der Barwert der damit

1) Vgl. Barro, R.J., Are Government Bonds Net Wealth?, in: Journal of Political Economy, 1974, S. $1095 \mathrm{ff}$; derselbe, Public Debt and Taxes, noch nicht veroffentlicht, o.J., S. $189 \mathrm{ff}$. 
verbundenen Steuerlasten $\left(\mathrm{T}^{\mathrm{B}}\right)$ als Passivum in die Vermögensbilanz des privaten sektors eingebucht werden:

$$
(2-2-25) \quad V=M Z+K+B-T^{B}
$$

Und unter sogenannten Ricardo-Bedingungen sind $B$ und $\mathrm{T}^{\mathrm{B}}$ gleichgroB: Der Barwert einer zeitlich unbegrenzten Steuerzahlung $\left(T^{B}\right)$ zur Bedienung der privaten Zinsansprüche an den Staat in Höhe von 1b.B je Haushaltsperiode beträgt, wenn $(1+i b)$ als Diskontierungsfaktor verwendet wird:

$$
(2-2-26) \quad T^{B}=\lim _{n \rightarrow \infty} \frac{1 b \cdot B}{(1+i b)^{n}} \cdot \frac{(1+1 b)^{n}-1}{i b}=B \text {. }
$$

Saldiert man dementsprechend, geht $(2-2-25)$ in die Vermögensgleichung

$$
(2-2-27) \quad V=M Z+K
$$

über. Konventionelle Vermögenseffekte bleiben dann bei einer kreditmarktfinanzierten Vermehrung der staatlichen Güternachfrage aus.

DaB der Lerner-Effekt entfällt, ist offensichtlich: Das Vermögensniveau bleibt konstant. DaB es darüberhinaus auch zu keinem zinseffekt kommt, obgleich doch der staat vermehrt staatspapiere emittiert, wird klar, wenn man folgendes bedenkt: Sofern ein vollständiges tax discounting stattindet, hat das staatliche Handeln für die Vermögensbilanz des privaten Sektors zwel Konsequenzen.

Zum einen entsteht in Höhe der Steuerverbindlichkeiten ein Passivposten. Zum anderen verlängert sich die Aktivseite um genau diesen Betrag. Ein vorher gleichgewich- 
tiges Portefeuille ist mithin aus der Ruhelage gebracht. Um die Diskrepanz zwischen tatsächlicher und gewünschter Portefeuillestruktur abzubauen, wird deshalb der Zuwachs an Aktiva in eine Verwendung gelenkt, die, nur mit umgekehrten Vorzeichen, der Passivmehrung entspricht. Es konzentriert sich folglich bei einem vollständigen tax discounting die zusätzliche Aktivanachfrage allein auf den Markt für staatspapiere. Dem zusätzlichen Angebot des Staates steht eine gleichgroBe Nachfrage der Privaten gegenüber, und es ist nur ein Mengenund kein Prelseffekt zu verzeichnen. Der Wunsch nach Diversifizierung des Aktivazuwachses, wie im Fall ohne tax discounting, wo also keine Veränderung bei den Passiva konstatiert worden ist, besteht jetzt nicht; mithin bleibt ein konventioneller Portfolioeffekt aus.

Die Diskussion darüber, ob von den staatsbürgern künftige Steuerzahlungen, die durch Staatsschulden bedingt sind, in ihren Vermögensbilanzen internalisiert werden, und wenn $j a$, in welchem AusmaB dies geschieht, ist alt und neuerdings wieder entflammt ${ }^{1}$. Sie wird auf theoretischer und empirischer Ebene geführt.

1) Vgl. für einen Uberblick neben den genannten Aufsätzen von Barro auch Gandenberger, 0., Zur Rationalität der öffentlichen Kreditnahme, in: Finanzarchiv, 1971, S. 369 ff.; Stevens, N.A., Government Debt Financing-Its Effects in View of Tax Discounting, in: Federal Reserve Bank of St. Louis Review, 1979, Nr. 7, S. 11 ff.; Räth, N., Die Zwangsanleihe als finanzpolitisches Instrument, St. Gallen, 1980, S. 104 ff.; Holcombe, R., Jackson, J.D., Zardkoohi, A., The National Debt Controversy, in: Kyklos, 1981, S. $186 \mathrm{ff.;}$ Buiter, W., Tobin, J., Debt Neutrality: A Brief Review of Doctrine and Evidence, zur Veröffentlichung vorgesehen in: Fürstenberg, G.M. von (Hrsg.), Social Security versus Private Saving in Post-Industrial Societies. 
Die theoretischen Erwägungen nehmen ihren Ausgang bei den Ricardo-Bedingungen ${ }^{1 !}$ sind sie erfüllt, konstatiert ein repräsentatives Wirtschaftssubjekt im Fall einer Plazierung von Staatspapieren im privaten Bereich keinen Vermögenszuwachs. Die wichtigsten der Ricardo-Bedingungen sowie Einwände und Gegeneinwände sind:

Erstens: Es besteht für ein repräsentatives Wirtschaftssubjekt keine Möglichkeit, sich der durch die staatsschulden bedingten steuerlast zu entziehen, sei es durch Auswanderung oder sei es durch Tod. Weil jedoch, so wird eingewendet, realiter solche steuerlasten nicht nur innerhalb der steuerpflichtigen Zeitspanne, sondern auch danach anfallen und ein repräsentatives Wirtschaftssubjekt dieses ignorieren könne, sei der Barwert der zukünftigen Steuerlasten, auch wenn sonst Ricardo-Bedingungen gelten, geringer als der staatsanleihebetrag.

Dem kann man mit Barro entgegenhalten 2), den Menschen sei gar nicht daran gelegen, sich auf Kosten ihrer Nachkommen $z u$ bereichern. Denn der Lebensnutzen eines Individuums hängt nicht nur vom eigenen Konsum, sondern auch vom Lebensnutzen seiner Nachfahren ab. Dieser kann nicht nur durch Weitergabe von staatsschulden beeinträchtigt, sondern auch durch Abtreten von Einkommensteilen während der gemeinsamen Lebenszeit und durch Nachlässe erhöht werden. Damit verschiebt sich der Planungshorizont in die zukunft und die Planungsperiode endlich lebender wirtschaftssubjekte reicht bis ins Unendliche. Anders gewendet: Weil Individuen die Belastung ihrer

1) Vgl. die Liste bei Barro, R.J., Public Debt and Taxes, a.a.0., s. 196 .

2) Vgl. ebenda, S. $197 \mathrm{ff}$. sowie derselbe, Are Government Bonds Net Wealth?, a.a.O., S. $1098 \mathrm{ff}$. 
Nachkommen als äquivalent der eigenen ansehen, ist unerheblich, wann $\mathrm{Zins-}$ und Tilgungsverpflichtungen anfallen ${ }^{1)}$ ?

Zweitens: Die Rate, mit der ein repräsentatives Wirtschaftssubjekt die durch die staatsschuld bedingten Steuerzahlungen diskontiert, gleicht dem zins auf Staatspapiere. Wenn dagegen die Diskontierungsrate über dem Zins auf staatstitel liegt, sind unter ansonsten Ricardoschen Bedingungen der Barwert der künftigen Steuerbelastung infolge der staatsverschuldung und der Betrag der emittierten Staatspapiere nicht mehr äquivalent, sondern es ist $T_{B}<B$.

DaB dies auch realiter so sei, wird von Pesek und Saving damit plausibel gemacht ${ }^{2}$, daB die Steuerpflicht zu wesentlichen Teilen an Erträgen aus menschlichem Vermögen anknüpft. Und weil Humanvermögen ein Gut mit nur begrenzter Haltbarkeit ist, würden auf damit verbundene, künftige Erträge und Aufwendungen eine im Vergleich zum sonstigen Vermögen höhere Diskontierungsrate angewendet. Wenn der $\mathrm{zins}$ auf Staatspapiere der Rate, mit der künftige Erträge und Aufwendungen des nicht-menschlichen Vermögens diskontiert werden, entspricht, folgt aus der Hyoothese von Pesek und Saving, daB die Rate, mit der zukünftige Steuerzahlungen abgezinst werden, gröBer ist, als der zins auf staatstitel.

1) "... finite lives will not be relevant to the capitalization of future tax liabilities so long as current generations are connected to future generations by a chain of operative intergenerational transfers". Derselbe, Are Government Bonds Net Wealth?, a.a.o., S. 1095 .

2) Pesek, B.P., Saving, T.R., Money, Wealth, and Economic Theory, London, New York, 1967, S. $262 \mathrm{ff}$. 
Drittens: Weder hinsichtlich der insgesamt anfallenden Steuern zur Finanzierung der Zins- und Tilgungsausgaben, noch hinsichtlich des indjviduellen Antells daran besteht ungewiBheit. Weil die Privaten in der Realität aber nicht wüBten, welche steuerlasten auf sie infolge der Staatsverschuldung zukämen oder einer "public debt illusion" unterlägen, so wird behauptet", würden die Steuerverbindlichkeiten, wenn überhaupt, dann nur unvollständig in die Vermögensbilanzen der Privaten internalisiert. In Gleichung (2-2-26) ist demnach das $B$ in der Barwertformel kleiner als jenes auf der rechten Seite und mithin $\mathrm{T}^{\mathrm{b}}<\mathrm{B}$.

Dem kann entgegengehalten werden, daB UngewiBheit und Illusion keineswegs notwendigerweise eine Unterschätzung implizieren: "One could just as well argue that government deficits, which are well publicized, make people nervous and induce them to feel poorer"2). In der Bundesrepublik Deutschland etwa deutet die gegenwärtig $\mathrm{zu}$ beobachtende Furcht vor einer möglichen währungsreform oder einem Staatsbankrott weniger auf eine Unter- sondern mehr auf eine Uberschätzung dieser Art hin. Dann kommt es, sofern ansonsten Ricardo-Bedingungen gelten, infolge einer Plazierung von staatsschuldtiteln in den Portefeuilles der Privaten gar zu einem Vermögensrückgang ${ }^{3}$ !

Nimmt man die theoretischen Erwăgungen zusammen, so spricht vieles dafür, daB ein partielles tax-discounting

1) Vgl. Gandenberger, 0., Zur Rationalität der öffentlichen Kreditnahme, a.a.0., s. $378 \mathrm{f}$.

2) Barro, R.J., Public Debt and Taxes, a.a.0., S. 202.

3) Im Fall eines übermäBigen tax discounting kehren sich die abgeleiteten Ergebnisse in ihr Gegenteil um. Via Lerner-Effekt wir die private Güternachfrage gedämpft, via Portfolioeffekt stimuliert. 
(in der Bundesrepublik Deutschland insbesondere aufgrund des letztgenannten Gesichtspunktes) stattfindet!' also die Privaten künftige Steuerzahlungen, die mit einer staatlichen Kreditaufnahme verknüpt sind, bei ihren Portfoliodisposition ins Kalkül ziehen. Insoweit kommt es zu keinen konventionellen (weder expansiven noch kontraktiven) Vermögenseffekten.

f) Staatsverschuldung und privates Vermögen: definitorischer Ansatz versus Sparansatz

Zur Bestimmung des Vermögens, welches sich infolge einer staatlichen Intervention bei den Privaten bildet (und auf dem Kapitalmarkt angelegt werden kann), werden in der Literatur zwei Wege beschritten ${ }^{2}$ ! Der erste, dem wir bisher gefolgt sind, ist der definitorische Ansatz. Ausgangspunkt dieser Vorgehensweise ist, daB sich das von den Privaten gehaltene Vermögen in den Vermögenskomponenten manifestiert. Die Vermögensmehrung innerhalb einer Periode ergibt sich mithin aus der Addition der Veränderung der einzelnen Portfoliopositionen. Wenn tax discounting, Vermögensumbewertungen, menschliches Vermögen und Giralgeld unberücksichtigt bleiben, gilt folglich (definitorischer Ansatz):

$(2-2-28) \mathrm{V}-\mathrm{V}_{-1}=\mathrm{MZ}-\mathrm{MZ}-1+\mathrm{B}-\mathrm{B}_{-1}+\mathrm{K}-\mathrm{K}_{-1} \cdot$

Demnach steigt ceteris paribus im Umfang des staatlichen

1) Empirische Untersuchungen sind von Kochin, von Yawitz und Meyer sowie von Tanner angestellt worden. Ihre Ergebnisse differieren erheblich und tragen deshalb wenig zur Klärung bei. Während Kochin eine Bestätigung für partielles und Tanner gar für vollständiges tax discounting fand, beobachteten Yawitz und Meyer keinerlei tax discounting. Für einen Uberblick vgl. Stevens, N.A., Government Debt Financing - Its Effects in View of Tax Discounting, a.a.0., S. $16 \mathrm{ff}$. sowie Buiter, W., Tobin, J., Debt Neutrality: A Brief Review of Doctrine and Evidence, a.a.0., S. $9 \mathrm{ff}$.

2) Vgl. Kennedy, P.E., Direct Wealth Effects in Macroeconomic Models: The Saving vs. the Definitional Approach, in: Journal of Money, Credit, and Bankging, 1978 , S. 94 ff. 
Kanitalmarktkredits $\left(B-B_{-1}\right)$ das Vermögen der Privaten.

Aus Sicht der Privaten nimmt dagegen das Vermögen nicht $z u$, wenn sie mehr staatspapiere halten, sondern, sofern die Einnahmen die Ausgaben übersteigen, sie also sparen (Sparansatz) :

$(2-2-29) \quad \mathrm{V}-\mathrm{v}_{-1}=\mathrm{s}$.

Demnach steigt das Vermögen der Privaten innerhalb einer Periode um den Teil des verfügbaren Einkommens, den die Privaten nicht konsumieren.

Ihren Vermögenszuwachs wünschen die Privaten auf die Portfoliokomponenten aufuteilen, es gilt also:

$$
(2-2-30) \quad V-V_{-1}=\left(B^{d}-B_{-1}^{d}\right)+\left(K^{d}-K_{-1}^{d}\right)+(L-L-1)
$$

In einer Gleichgewichtslage stimmt sowohl im Hinblick auf die Struktur als auch im Hinblick auf das Niveau das tatsächliche (= angebotene) Portefeuille mit dem gewünschten Portefeuille überein. Nimmt man die Gleichungen (2-2-28) und (2-2-30) in ÄnderungsgröBen, gilt also:

$(2-2-31) \quad d B^{s} / d K^{s} / d M z=d B^{d} / d K^{d} / d L$

und

$(2-2-32) \quad d B^{s}+d K^{s}+d M Z=d B^{d}+d K^{d}+d L$.

Aus den Gleichungen (2-2-31) und (2-2-32) wird folgendes deutlich: Bei $\mathrm{dMZ}=0$ konkurrieren private Unternehmen und der staat mit ihren zinstragenden Aktiva $\left(d K^{S}\right.$ und $\left.d B^{S}\right)$ um eine Aufnahme in die Portefeuilles der Privaten. Sie setzen beide ihre wünsche nur dann zu konstanten zinsen durch, wenn (a) das Portefeuille der Privaten um den Betrag des zusätzlichen Angebots steigt und (b) die Privaten den Vermögenszuwachs genau so auf die einzelnen Portfoliokomponenten auf- 
teilen wollen, wie sich das Angebot erhöht. Bislang wurde (vollständiges tax discounting ausgenommen) stets unterstellt, daB Annahme (a) zutrifft, nicht aber Annahme (b). Zinserhöhungen wurden also mit Diversifizierungserwägungen der Vermögensanleger plausibel gemacht.

Im Silber-Model $1^{1)}$ z.B. bestand das Portefeuille der Privaten aus den zwei Vermögenstiteln Zentralbankgeld und Staatspapiere. Infolge einer Emission von Staatstiteln galt: $d B^{s}=d B^{d}+d L$ und $d L>0$, so $d a B$ es auf dem Markt für staatspapiere $z u$ einem AngebotsüberschuB kam $\left(\mathrm{dB}^{s}>\mathrm{dB}^{\mathrm{d}}\right)$ und deswegen der Zins auf Staatspapiere stieg. Anders gesehen: Ist $d L=0$, folgt $d B^{S}=d B^{d}$, und der $z$ ins auf staatspapiere bleibt konstant.

$\mathrm{Zu}$ einer $\mathrm{Zinserhöhung} \mathrm{kommt} \mathrm{es} \mathrm{aber} \mathrm{dennoch,} \mathrm{und} \mathrm{zwar}$ dann, wenn sich das gewünschte Portefeuille der Privaten um einen geringeren Betrag als das Angebot an zinstragenden Aktiva vergröBert, wenn also Annahme (a) aufgehoben wird. In diesem Fall entsteht auch bei $d L=0$ auf dem Markt für staatspapiere ein AngebotsüberschuB, und die $Z$ insen steigen. Ist gar $d L>0$, erhöhen sich die zinsen um so mehr.

Die Gleichungen (2-2-28) und (2-2-29) machen deutlich, daB die Annahme, infolge einer staatlichen Kreditaufnahme würde sich das Portefeuille um den Betrag der Staatspapiere ausdehnen, nur dann zutrifft, wenn die Veränderungsbeträge der sonstigen Portefeuillekomponenten konstant bleiben (definitorischer Ansatz), anders gesehen: wenn die Privaten um den Betrag der staatlichen Kreditaufnahme mehr sparen (Sparansatz). Erhöht sich die Ersparnis um weniger, als der staat Kredite nachfragt, kommt es nicht nur aus Gründen der Diversifikation des Vermögenszuwachses, sondern auch wegen des $\mathrm{zu}$ geringen Umfangs des Vermögenszuwachses zu einer Zinserhöhung. Das konventionelle Portfolio-crowding-out fällt dann entsprechend größer aus.

1) Vgl. S. $61 \mathrm{ff}$. oben. 
4. Exkurs: Quellen- versus Fontänentheorie

\section{a) Darstellung}

Durch einen Aufsatz von Stützel im Jahre 1978 ${ }^{1)}$ angeregt, ist in der Bundesrepublik Deutschland in den letzten Jahren die Diskussion darüber wieder aufgelebt ${ }^{2}$, welches die adäquate theoretische Vorstellung sei, wenn es um die Abschätzung einer möglichen Verdrängungskonkurrenz zwischen staat und Privaten auf dem Kapitalmarkt infolge einer staatlichen Kreditaufnahme geht.

Die Debatte wird geführt zwischen (in der Terminologie Stützels) Fontänentheoretikern und Quellentheoretikern. Stützel gehört der Gruppe der Fontänentheoretiker an. Seiner Ansicht nach "ist die Ausgabenwirtschaft des Staates mit einer Fontäne vergleichbar, die mitten im Teich steht: Unten saugen die Pumpen die liquiden Mittel vom Teich ab; oben werden sie als Springbrunnen über die ganze Bevölkerung verteilt, fallen also damit wieder in den Teich zurück. Indem der Staat das Geliehene ausgibt, nährt er selbst den Kreditmarkt, reichert ihn

1) Vgl. Stützel, W., Ober- und Untergrenzen der öffentlichen Verschuldung, in: Kredit und Kapital, 1978, S. 444 ff.

2) Vgl. Francke, H. - H., Ober- und Untergrenzen der öffentlichen Verschuldung. Ein kritischer Kommentar zu Ausfuhrungen von Wolfgang Stützel, in: Kredit und Kapital, 1979, S. 362 ff.; Dieckheuer, G., Der Crowding-out-Effekt - zum gegenwärtigen Stand von Theorie und Empirie, a.a.O., S. 140 f.; Pohl, Reinhard, Die Auswirkungen der Staatsverschuldung auf die Finanzmärkte, in: Beihefte der Konjunkturpolitik, Nr. 27, 1980 , S. 85 ff.; Teschner, M., Hält die Crowdingout-These kreislauftheoretischer Kritik stand?, in: Vierteljahreshefte zur Wirtschaftsforschung, 1980, S. $121 \mathrm{ff.;}$ Duwendag, D., Monetäre Grenzen der Staatsverschuldung, in: Duwendag, D., Siebert, H. (Hrsg.), Politik und Markt, Wirtschaftspolitische Probleme der 80 er Jahre, Stuttgart, New York, 1980, S. $65 \mathrm{ff}$. 
um Anlage suchende Mittel an. Die Möglichkeiten privater Kreditaufnahme werden dadurch per saldo nicht im mindesten geschmälert"1) In der sichtweite stützels ist mithin "bei der Finanzierung stelgender Staatsdefizite nicht mit quantitativen Problemen zu rechnen"2) Denn "mit jedem AusgabenüberschuB des staates flieBen den Privaten als Inhaber der entsprechenden Einnahmeüberschüsse Gelder zu, die wiederum neue Anlage suchen" 3 !

Quellentheoretiker haben dagegen die Vorstellung "als sei der Kapitalmarkt eine Quelle, die eine bestimmte, unabhängig festliegende jährliche schüttung an liquiden Mitteln bringt. Wenn nur einer, der Bund, mehr davon absaugt, so bleibt für die anderen nur weniger übrig" 4 !

Worum es im Kern zwischen Quellen- und Fontänentheoretikern geht, ist offensichtlich folgendes: Durch eine Emission von Staatsschuldtiteln werden finanzielle Mittel vom staat absorbiert, die im privaten sektor ansonsten zur Finanzierung von Investitionen hätten verwendet werden können. Der staat verausgabt jedoch die Mittel wieder und induziert damit Expansionstendenzen, im Zuge dessen die Privaten auch zusätzliches Vermögen bilden und dieses auch am Kapitalmarkt anlegen. Fraktionsbildend ist die Antwort auf die Frage nach dem AusmaB dieser Anlage. Fontänentheoretiker nehmen an, daB ein Saysches Theorem auf dem Kapitalmarkt gilt, das zusätzliche staatliche Wertpapierangebot sich also eine Nachfrage in glelcher Hohe schafft; Quellentheoretiker verneinen dies.

1) Stützel, W., Ober- und Untergrenzen der öffentlichen Verschuldung, a.a.0., s. 446 .

2) Ebenda, S. 446 .

3) Ebenda, S. 449 .

4) Ebenda, S. 445 . 
b) Bedingungen der Fontänentheorie

Bei konstanter Zentralbankgeldmenge, d.h. $d M z=0$, bietet sich unter Verwendung (der nun in AnderungsgröBen geschriebenen) Gleichungen (2-2-28) und (2-2-29) folgende Interpretation an. Bedingung dafür, dab die hinter der Quellentheorie stehende Vorstellung stimmt, ist:

(2-2-33) $d B=-d K$ und $d S=0$.

Der AusgabenüberschuB des Staates geht in voller Hobe zu Lasten der privaten Investitionsausgaben. Der EinnahmeüberschuB des privaten Sektors steigt, weil die privaten Investoren um den Betrag der staatlichen Kapitalmarktverschuldung ihre Investitionspläne vertagen.

Bedingung dafür, daB die hinter der Fontänentheorie stehende Vorstellung stimmt, ist:

$(2-2-34) d S=d B$ und $d K=0$.

Der AusgabenüberschuB des Staates wird ausschlieBlich durch zusätzliche private Ersparnis finanziert.

Hieraus folgt: Zwischen Fontänentheoretikern und Quellentheoretikern ist unbestritten, daB dem Haushaltsdefizit, des Staates ein gleichgroBer EinnahmeüberschuB im privaten Sektor gegenübersteht. Dies ist eine saldenmechanische Trivialität. Kontrovers beurteilt wird, ob dieser EinnahmeüberschuB dadurch zustandekommt, daB vermehrt gespart wird oder dadurch, daB vermindert investiert wird.

Damit ersteres eintritt und mithin die Bedingung (2-2-34) erfüllt ist, müssen sich die Gesamtausgaben infolge des staatlichen Handelns nach MaBgabe des reziproken Wertes der auf das Sozialprodukt $Y$ bezogenen marginalen sparquote $\left(S_{Y}\right)$ erhöhen: 


$$
(2-2-35) \quad \frac{d Y}{d G}=\frac{1}{S_{Y}} .
$$

Dann nämlich ist ${ }^{1)}$ :

$$
(2-2-36) \quad d B=d G=S_{Y} \cdot d Y=d S=d B .
$$

Im neuen Stromgleichgewicht wird von den Privaten genau soviel zusätzlich gespart, wie der Staat seine Kreditaufnahme ausgedehnt hat. Die Fontänentheorie gilt also nur unter der Bedingung, daB das Gesamtausgabenniveau nach MaBgabe des "einfachen" keynesianischen Multiplikators steigt.

Allfälligen monetären Bremseffekten, wie dem Hicksschen crowding-out-Effekt und dem konventionellen Portfoliocrowding-out-Effekt, messen die Fontänentheoretiker folglich keine Bedeutung bel. Stiegen nämlich die Gesamtausgaben nach Maßgabe des "einfachen" keynesianischen Multiplikators, würden die Privaten im neuen Stromgleichgewicht konstatieren: Ihr Portefeuille hat sich um den Betrag der zusätzlichen Ersparnisse vergröBert. Sie halten in Höhe dieses Betrages mehr Staatspapiere. Zudem muB ein höheres güterwirtschaftliches Transaktionsvolumen bewältigt werden. Deshalb ist die tatsächliche Portfoliostruktur im Vergleich zur gewünschten zu illiquide; es werden Anpassungsreaktionen ausgelöst, im zuge derer sich die zinsen erhöhen und die privaten Investitionen zurückgedrängt werden. Um den Betrag der verdrängten Investitionen wird die staatliche Kreditaufnahme nicht durch vermehrtes Sparen, sondern durch verminderte Kreditvergabe an die privaten Investoren finanziert.

Im Ausma $B$ des Hicksschen crowding-out und des konventionellen Portfolio-crowding-out besorgt sich also der staat finanzielle Mittel auf dem Kapitalmarkt zu Lasten privater Investitionen. Diese beiden monetären Bremseffekt werden von

1) Aus Vereinfachungsgründen wird vernachlässigt, daB im Zuge eines expansiven Prozesses auch die Steuern steigen; es gilt $d T=0$ und $d G=d B$. 
den Fontänentheoretikern vernachlässigt.

Allgemein: Fontänentheoretiker sind nicht nur der Meinung, daB monetären Bremseffekten bel elner kreditär finanzierten Ausdehnung der Staatsausgaben keine nennenswerte Bedeutung beikommt, sondern sie gehen ganz generell von der aggregativen Effizienz des finanzwirtschaftlichen Handelns aus. Denn damit die Fontänentheorle zutrifft, muB das Sozialprodukt nach MaBgabe der marginalen Sparquote um ein Mehrfaches des staatlichen Primärimpulses steigen (und zwar nicht nur nominell, sondern real). Was zum Belspiel die gesamtwirtschaftliche Ausgangskonstellation betrifft, setzt dies voraus, daB die Ursachen für die Unterbeschäftigung nicht auf der Angebotsseite liegen. Bei einer angebotsseitig bedingten Unterbeschäftigung bleibt nämlich trotz staatlicher Nachfrageimpulse das reale Sozialprodukt konstant. Mithin vergröBern sich infolge des staatlichen Handelns die realen Ersparnisse der Privaten nicht, und der staat kann seine (in Gütern gemessene) Kreditwünsche nur zu Lasten von privaten Investitionen durchsetzen.

B. Sonstige Vermögenseffekte

1. Ursachen und Transmissionskanăle von Vermögenseffekten

Die bisherigen Uberlegungen in diesem Kapitel haben an dem Vorwurf der Monetaristen angeknüpft, Keynesianer würden den EinfluB einer Emission von Staatspapieren im Zuge einer Erhöhung der Transformationsausgaben auf das Ausgabenverhalten der Privaten unterschätzen. Neben den damit verbundenen konventionellen Vermögenseffekten (die ihre Ursache in einer Absorption von Staatspapieren in den Portefeuilles der Privaten haben) gibt es noch eine Vielzahl anderer Vermögenseffekte, welche ihren Ausgang ebenfalls bei dem finanzwirtschaftlichen Handeln nehmen können. Diese sind Gegenstand der folgenden Erörterung. 
Hinsichtlich ihrer Ursachen kann man Vermögenseffekte danach unterscheiden ${ }^{1}$, ob sie auf einer mengenmäBigen Bestandsänderung einzelner Vermögensobjekte (Vermöggensvolumeneffekt) oder auf einer Umbewertung der konstanten Bestände einzelner Vermögensarten (Vermögensbewertungseffekt) beruhen. Bei einem Vermögensvolumeneffekt stellt mithin die Mengenkomponente, bei einem Vermögenswerteffekt die Preiskomponente des privaten Vermögens das Bindeglied zwischen den finanzwirtschaftlichen Primärimpulsen und den privaten Ausgaben dar 2)

Vermögensänderungen wirken sich (wie schon bei den konventionellen Vermögenseffekten) auf zwei Wegen auf das Ausgabeverhalten der Privaten aus: über die VermögenKonsum-Relation und über die Portfolio-Allokation-Relation ${ }^{3}$ ! Beide tbertragungsmechanismen kommen keineswegs stets gleichzeitig zum Wirken. Denn erstgenannter beruht auf einer Veränderung des Vermögensniveaus (Vermögensniveaueffekt), während zweitgenannter Folge von Disparitäten zwischen der gewünschten und der tatsächlichen Struktur des Portfolios ist (Vermögensstruktureffekt). Vermögensniveaueffekte beeinflussen den privaten Konsum, Vermögensstruktureffekte die zinsabhängigen Ausgaben der Privaten, d.h. hauptsächlich die privaten Investitionen.

1) Diese Differenzierung stammt von Meyer und Yawitz, die allerdings die Begriffe direkte (=Volumen) und indirekte (=Bewertung) Vermögenseffekte verwenden; vgl. Meyer, L.H., Wealth Effects and the Effectiveness of Monetary and Fiscal Policies, a.a.O., S. $484 \mathrm{ff}$.; Yawitz, J., Economically Relevant Wealth: Its Specification and Role in Consumption, a.a.0., s. 22 ff.

2) Die beiden schon abgehandelten konventionellen Vermögenseffekte, der konventionelle Portfolioeffekt und der Lerner-Effekt, sind Folge einer Vermögensmengenmehrung einer Portfoliokomponente, den Staatspapieren, und sind deshalb der Kategorie der Vermögensmengeneffekte zuzurechnen.

3) Vgl. Meyer, L.H., Wealth Effects and the Effectiveness of Monetary and Fiscal Policies, a.a.o., s. $481 \mathrm{ff}$. 
Weil mit zunehmendem Vermögen die Privaten mehr konsumieren, handelt es sich um kontraktive Vermögensniveaueffekte, wenn das private Vermögen abnimmt und die Privaten deshalb ihre Konsumausgaben einschränken. Durch Vermögensstruktureffekte werden die privaten Investitionen verdrängt, wenn der die Investitionspläne der Privaten determinierende zins steigt. Wir gehen davon aus, daB (erstens) die Geldnachfrage vermögensabhängig ist und (zweitens) alle Nicht-Geld-Vermögenskomponenten aus sicht der Vermögensanleger in einer derartigen Beziehung zueinander stehen, daB sich ihre Renditen gleichgerichtet entwickeln. Dann steigen die zinsen immer dann, wenn in den Portefeuilles der Privaten die Nicht-Geld-Vermögenskomponenten in Relation zur Kasse zunehmen, und die zinsen sinken immer dann, wenn sich die Portfoliostruktur zu Lasten der Nicht-Geld-Vermögenskomponenten und zugunsten der Kassenhaltung verändert.

Der TransmissionsprozeB finanzwirtschaftlicher Primärimpulse via Vermögenseffekte wird in der Ubersicht 7 schematisch zusammengefaBt ${ }^{1}$ ?

2. Vermögensmengeneffekte

Vermögensmengeneffekte beruhen auf einer mengenmäBigen Bestandsveränderung einzelner Vermögenskomponenten. In unseren tberlegungen haben bisher unterschiedlich viele - niemals jedoch sämtliche - Komponenten des privaten Vermögens Berücksichtigung gefunden. Zieht man alle Komponenten in Betracht, kann das Vermögen der Privaten als Summe aus menschlichem (Humanvermögen) und nicht-menschlichem Vermögen definiert werden, wobei letzteres in seine beiden Teile Geldvermögen und Sachvermögen (Real-

1) Vgl. ähnlich Metzger, R., Zur Effizienz und Interdependenz der Geld- und Fiskalpolitik, a.a.o., S. 34 . 
Ubersicht 7: Ursachen und Transmissionskanäle von Vermögenseffekten

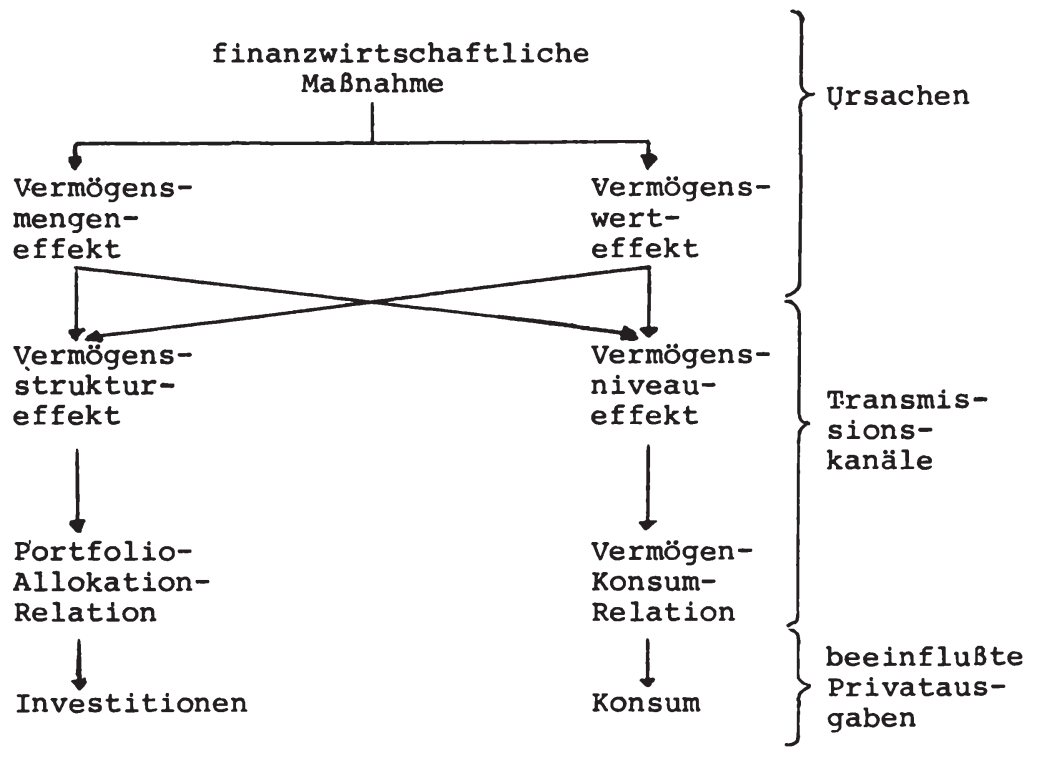

kapital) zerfällt. Dementsprechend können Sach-, Humanund Geldvermögensmengeneffekte unterschieden werden.

Der Sachvermögensmengeneffekt infolge einer finanzwirtschaftlichen Intervention ist der Saldo aus deswegen unterlassenen und deshalb getätigten privaten Investitionen. Ist er positiv, erhöht sich (bei Konstanz aller anderen Vermögenskomponenten) das Vermögensniveau, und verschiebt sich die Portefeuillestruktur zugunsten der Nicht-GeldVermögenskomponenten und $\mathrm{zu}$ Lasten des Geldes. Durch einen positiven Sachvermögensmengeneffekt werden folglich die privaten Ausgaben via Vermögen-Konsum-Relation stimuliert und via Vermögen-Allokation-Relation gedämpft. 
Das Humanvermögen wird in der Literatur bei einer Vermögensdefinition meist ausgeschlossen; deswegen haben Humanvermögenseffekte bislang wenig Beachtung gefunden. Inwieweit der mengenmäBige Bestand an Humanvermögen der Privaten infolge einer kreditfinanzierten Erhöhung der Transformationsausgaben steigt, ist von der Art der Staatsausgaben abhängig. Ein Zuwachs ist beispielsweise bei einer Verwendung der Mittel im Bildungsbereich zu erwarten. Dann ändert sich zum einen die Struktur des Vermögens der Privaten zugunsten der Nicht-Geld-Vermögenskomponenten, und zum anderen erhöht sich das Vermögensniveau. Wie bei einem positiven Sachvermögensmengeneffekt entsteht für die Privaten ein Anreiz, ihren Konsum auszuweiten und ihre (sachlichen) Investitionen einzuschränken.

In welchem Umfang Geldvermögensmengeneffekte durch eine auf dem Kreditmarkt finanzierte Ausdehnung der staatlichen Güternachfrage induziert werden, wird nicht zuletzt dadurch bestimmt, was alles zum Geldvermögen der Privaten gehört. Hierüber besteht in der Literatur keine Einigkeit ${ }^{1}$ ? Die folgenden Uberlegungen lassen sich anhand einer Matrix illustrieren, mit Hilfe derer das Bruttogeldvermögen des privaten Sektors nach den Kriterien Schuldner (Reihen) und Verzinsung (Zeilen) strukturiert worden ist (vgl. Ubersicht 8 ).

Nach einer Sichtweise, welche insbesondere mit den Namen Gurley und Shaw verbunden ist, sind Forderungstitel in Händen der Privaten zwar stets Bestandteile des privaten Bruttovermögens, aber nur dann auch des privaten Nettovermögens, sofern ihnen

1) Für einen tberblick vgl. Hoyer, W., Vermögenseffekte des Geldes, Frankfurt am Main, Bern, Las Vegas, 1978, S. $18 \mathrm{ff}$. 
Ubersicht 8: Geldvermögenskomponenten

\begin{tabular}{|c|c|c|}
\hline Schuldn & $\begin{array}{l}\text { Staatssektor } \\
\text { (outside } \\
\text { financial } \\
\text { asset) }\end{array}$ & $\begin{array}{l}\text { Privatsektor } \\
\text { (inside } \\
\text { financial } \\
\text { asset) }\end{array}$ \\
\hline zinstragend & $\begin{array}{l}\text { Staatspapiere } \\
\text { (1. konventio- } \\
\text { neller Ver- } \\
\text { mögenseffekt } \\
\text { 2. tax } \\
\text { discounting) }\end{array}$ & $\begin{array}{l}\text { zinstragende } \\
\text { Forderungen } \\
\text { an Private }\end{array}$ \\
\hline zinslos & $\begin{array}{l}\text { Zentralbankgeld } \\
\text { (outside money) }\end{array}$ & $\begin{array}{l}\text { Giralgeld } \\
\text { (inside money, } \\
\text { Pesek/Saving- } \\
\text { Effekt) }\end{array}$ \\
\hline
\end{tabular}

keine entsprechende Verbindlichkeit innerhalb des privaten Sektors gegenubersteht (outside financial asset) . Andernfalls heben sich nämlich bei der Aggregation der einzelnen Vermögenskonten des privaten Sektors diese Forderungen mit den korrespondierenden Verbindlichkeiten auf (inside financial asset). Demnach besteht das Nettogeldvermögen der Privaten also nur aus Staatspapieren und Zentralbankgeld. Bei Konstanz der Geldbasis kann durch kreditfinanzierte zusätzliche staatsausgaben lediglich ein einziger Geldvermögensmengeneffekt induziert werden: der konventionelle Vermögenseffekt. DaB auch dieser entfällt, wenn ein vollständiges tax discounting stattfindet, ist schon dargelegt worden. 
Diese buchführungstechnisch begründete Verneinung einer Vermögenseigenschaft von internen Forderungen wird, was das Giralgeld betrifft, von den Vertretern der "Money-as-Net-Wealth-Hypothesis" zurückgewiesen. Nach ihrer mehr ökonomisch orientierten Betrachturigiweise - im AnschluB an Uberlegungen von Pesek und Saving verdeckt die Unterscheidung zwischen vermögensunwirksamem inside money und vermögenswirksamem outside money die Tatsache, daB Giralgeldschöpfung profitable Aktivgeschäfte möglich macht und sich dadurch das vermögen der Geschäftsbanken bzw. das ihrer Eigentümer erhöht. Der Vermögenszuwachs infolge einer Giralgeldschöpfung ergibt sich aus der Differenz zwischen dem Barwert der Erlöse aus dem Aktivgeschäft und dem Barwert der Kosten der Giralgeldproduktion, d.h. eventuellen $\mathrm{zinsen}$ auf Depositen sowie Verwaltungskosten. Dieser Vermögenseffekt wird als Pesek/Saving-Effekt ${ }^{1}$ ) bezeichnet.

Kommt es im Zuge einer Kreditmarktfinanzierung von zusätzlichen Staatsausgaben (abweichend von der ansonsten gemachten Annahme) zu einer Erhöhung der Geldbasis und nimmt deshalb die Giralgeldmenge $z u$, ist mit einem expansiven Pesek/Saving-Effekt zu rechnen. Denn zum einen verwenden die Privaten wegen des Vermögenszuwachses einen vergleichsweise gröBeren Einkommensteil für Konsumausgaben. Zum anderen sinken die Zinsen. Zwar wollen die Vermögensanleger einen Teil des durch die Giralgeldschöpfung erfahrenen Vermögenszuwachses in Form von Kasse halten; die Geldnachfrage steigt also. Gleichzeitig erhöht sich aber um den Gesamtbetrag des zusätzlichen Giralgeldes das Geldangebot, so daB per Saldo ein Geldangebotsüberschuß entsteht.

1) Vgl. Meyer, L.H., Wealth Effects and the Effectiveness of Monetary and Fiscal Policies, a.a.o., S. 484; Yawitz, J., Economically Relevant Wealth: Its Specification and Role in Consumption, a.a.O., S. 23 . 


\section{Vermögenswerteffekte}

Vermögenswerteffekte resultieren aus einer Neubewertung der mengenmäBig konstanten Bestände von Vermögenskomponenten. Wir berücksichtigen im folgenden nur Zentralbankgeld, Staatspapiere sowie privates Sachkapital. Meyer unterscheidet drei Arten: zinsinduzierte, preisinduzierte und einkommensinduzierte Vermögenswerteffekte ${ }^{1 !}$ ?

Die Meyersche Differenzierung kann man gut anhand der kapitaltheoretisch orientierten Definition des Vermögens plausibel machen:) Demnach gehört zum Vermögen der Privaten alles, was Quelle künftiger Erträge ist. Der Realwert des privaten Vermögens $\left(v^{r}\right)$ ergibt sich aus der Addition der mit dem Diskontierungssatz i auf den Gegenwartszeitpunkt abgezinsten und preisbereinigten (P) Ertragsströme des Zentralbankgeldes ( $\mathrm{E}^{\mathrm{MZ}}$ ), der Staatspapiere $\left(E^{B}\right)$ sowie des Sachkapitals $\left(E^{K}\right)$ :

$$
(2-2-37) \quad V^{r}=\frac{\left(E^{M Z}+E^{B}+E^{K}\right)}{P \cdot i} .
$$

Es gibt mithin drei Variable, durch deren veränderung Umbewertungen verursacht werden können: Zinssatz (Diskontierungsfaktor), Preisniveau (Deflator) sowie die Erträge, und es lassen sich dementsprechend mit Meyer drei Arten von Vermögenswerteffekten unterscheiden.

Variiert infolge der finanzwirtschaftlichen Intervention das Preisniveau, wird ein preisinduzierter Vermögenswerteffekt bewirkt. Dafür kommen jedoch nur solche Vermögenskomponenten in Betracht, deren

1) Vgl. Meyer, L.H., Wealth Effects and the Effectiveness of Monetary and Fiscal Policies, a.a.O., S. 486.

2) Vgl. Hoyer, W., Vermögenseffekte des Geldes, a.a.0., S. 43 ff. 
nominelle Ertragsströme sich nicht gleichzeitig mit dem Preisniveau ändern. Dies sind hauptsächlich die beiden finanziellen Vermögenskomponenten Zentralbankgeld und Staatspapiere.

Steigt das Preisniveau, werden durch einen preisinduzierten Vermögenswerteffekt sowohl private Konsumals auch Investitionsausgaben verdrängt ${ }^{1 !}$. Denn da der Realwert der finanziellen Vermögenskomponenten und damit das reale Vermögen insgesamt sinkt, verwenden die Privaten nur noch einen geringeren Teil ihres verfügbaren Einkommens für Konsumzwecke. Weil der Realwert des Sachvermögens konstant bleibt, nimmt der reale wert der Nicht-Geld-Vermögenskomponenten relativ weniger ab als der reale Wert des Zentralbankgeldes. Die Portfoliostruktur ist mithin vergleichsweise illiquide. Dieser GeldnachfrageüberschuB treibt den $\mathrm{zins}$ in die Höhe und verdrängt private Investitionen.

Ein zinsinduzierter Vermögenswerteffekt kommt zustande, wenn sich der $\mathrm{Zins}$ ändert und deswegen die Vermögenshalter künftige Erträge mit einem höheren oder geringeren Diskontierungsfaktor abzinsen. Allerdings gibt es neben Vermögensgütern vom "fixed coupon-variable price"-Typ l= Vermögensgüter mit einem festen Nominalertrag und einem variablen Wert), wie zum Beispiel Staatspapiere, auch Vermögensgüter vom "variable coupon-fixed price"-Typ ( = Vermögensguter mit einem variablen Nominalertrag und einem festen wert) ${ }^{2)}$. Zinsvariationen schlagen sich in deren Bewertung nicht nieder. Hierzu gehört hauptsächlich das Zentralbankgeld.

1) Vgl. Currie, D., Monetary and Fiscal Policy and the Crowding-Out Issue, a.a.O., S. $19 \mathrm{f}$; für eine formale Analyse vgl. Metzger, R., Zur Effizienz und Interdependenz der Geld- und Fiskalpolitik, a.a.o., S. $109 \mathrm{ff}$.

2) Vgl. zu dieser Differenzierung Meyer, L.H., Wealth Effects and the Effectiveness of Monetary and Fiscal Policies, a.a.O., S. 487 f. Christian Schiller-978-3-631-75197-8 
Bei Berücksichtigung eines zinsinduzierten Vermögenswerteffektes wird über die Vermögen-Konsum-Relation die inverse Zinsabhängigkeit der privaten Ausgaben vergröBert. Die stabilisierungspolitische wirksamkeit einer kreditfinanzierten Erhöhung der Transformationsausgaben wird hierdurch gemindert. Uber die Vermögen-Allokation-Relation wird sie dagegen erhöht!) Denn die Wirtschaftssubjekte halten nach einer Zinserhöhung vergleichsweise zuviel Geld und zuwenig Nicht-GeldVermögensobjekte. Es entsteht ein Geldangebotsüberhang und damit eine zinssenkungstendenz, was private Investitionen anregt.

Der einkommensinduzierte Vermögenswerteffekt hat seine Ursache in einer Veränderung des nominellen Ertragsstromes des Sachvermögens infolge einer Variation seines Auslastungsgrades ${ }^{2}$ ). Gelingt es, durch das finanzwirtschaftliche Handeln den Auslastungsgrad des Sachvermögens zu erhöhen, nehmen die Erträge des Sachkapitals zu, und der Wert der Eigentumsrechte an Sachkapital steigt. Wegen des dadurch gestiegenen Niveaus des privaten Vermögens konsumieren die Privaten mehr. Gleichzeitig halten sie im Vergleich zum status quo ante zuviel Vermögen in Form von Nicht-Geld-Vermögenskomponenten und zuwenig Geld. Der GeldnachfrageüberschuB treibt das Zinsniveau nach oben; die zinsabhängigen Ausgaben der Privaten werden zu-

1) Vgl. Currie, D., Monetary and Fiscal Policy and the Crowding-Out Issue, a.a.O., S. $18 \mathrm{f}$; für eine formale Analyse vgl. Metzger, R., Zur Effizienz und Interdependenz der Geld- und Fiskalpolitik, a.a.0., S. 102 ff.; vgl. auch Zwick, B., "Snapback" and "Crowding out" Effects in Monetary and Fiscal Policy: Explanation and Interrelation, in: Journal of Money, Credit and Banking, 1974, S. $559 \mathrm{ff}$.

2) Vgl. Meyer, L.H., Wealth Effects and the Effectiveness of Monetary and Fiscal Policy, a.a.O., S. $488 \mathrm{f}$. 
rückgedrängt ${ }^{1}$ ?

In tbersicht 9 wird zusammengefaBt, wie die Privaten mit ihren Konsumausgaben und Investitionsausgaben auf Vermögensmengeneffekte und Vermögenswerteffekte reagieren.

Ubersicht 9: Vermögenseffekte und private Ausgaben

\begin{tabular}{|c|c|c|c|}
\hline & & Konsum & $\begin{array}{l}\text { Investi- } \\
\text { tionen }\end{array}$ \\
\hline \multirow{4}{*}{$\begin{array}{l}\text { Vermögens- } \\
\text { mengeneffekte }\end{array}$} & Sachvermögen & 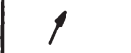 & $\downarrow$ \\
\hline & Humanvermögen & 1 & $\downarrow$ \\
\hline & Staatspapiere & 1 & $d$ \\
\hline & Geld & 1 & $f$ \\
\hline \multirow{3}{*}{$\begin{array}{l}\text { Vermögens- } \\
\text { werteffekte }\end{array}$} & Preisniveau & $b$ & $\downarrow$ \\
\hline & Zinsniveau & $d$ & $\varphi$ \\
\hline & $\begin{array}{l}\text { Auslastung des } \\
\text { Sachvermögens }\end{array}$ & 1 & $d$ \\
\hline
\end{tabular}

1) Vgl. Currie, D., Monetary and Fiscal Policy and the Crowding-Out Issue, a.a.0., S. 19; für eine formale Analyse vgl. Metzger, R., Zur Effizienz und Interdependenz von Geld- und Fiskalpolitik, a.a.0., S. $107 \mathrm{f}$. 
Drittes Kapitel: Ausgabeneffekte

A. Gegenstand der Uberlegungen

Zusatzliche Transformationsausgaben regen - für sich genommen - die Ausgabenaktivität der Privaten an. DaB ein derartiger funktionaler Zusammenhang besteht, ist wenig umstritten. Kontrovers wird allerdings die Veränderung der privaten Ausgaben infolge einer Transformationsausgabenerhöhung - sprich: der numerische Wert der partiellen Ableitung dieser Funktion - beurteilt. Je geringer dieser Wert ist, je schwächer also die durch die Transformationsausgaben induzierten pulling-in-Teileffekte ausfallen, um so wahrscheinlicher wird, daB die crowding-out-Teileffekte die Oberhand gewinnen und deshalb die Privaten im Vergleich zur Situation ohne finanzwirtschaftliche Intervention weniger Ausgaben tätigen.

Wie sich im Grundmodell zeigt, steigen, wenn der staat vermehrt Güter nachfragt, die gesamtwirtschaftlichen Ausgaben aus zwei Gründen: Zum einen nehmen uno actu mit der Staatsausgabenerhöhung die gesamtwirtschaftlichen Ausgaben um den Betrag der zusätzlich getätigten Transformationsausgaben additiv zu (direkter staatsausgabeneffekt) 1). Zum anderen stellen die Zusatzausgaben des Staates für die privaten Haushalte Einkommen dar und induzieren damit multiplikativ eine Erhöhung ihrer Konsumausgaben (einkommensinduzierter Konsumeffekt).

Häufig wird ein weiterer, die gesamtwirtschaftlichen

1) Vgl. Modigliani, F., Ando, A. Impacts of Fiscal Actions on Aggregate Income and the Monetarist Controversy: Theorey and Evidence, a.a.0., S. 23. 
Ausgaben vermehrender Sekundäreffekt angeführt: Die zusätzliche Güternachfrage von Staat und privaten Haushalten erhöht den Auslastungsgrad des Sachkapitals und macht, so wird behauptet, Erweiterungen des Sachkapitals rentabel. Eine Transformationsausgabenvermehrung regt mithin die Unternehmen an, ihre Investitionspläne aufzustocken (Akzelerationseffekt).

Damit ist das Untersuchungsfeld dieses Kapitels abgesteckt. Erörtert wird, was dafür spricht, daB ein Transformationsausgabenimpuls - für sich genommen - nicht zu dem erhofften direkten staatsausgabeneffekt, einkommensinduzierten Konsumeffekt und Akzelerationseffekt führt.

Die Analyse wird daher erstens auf die Betrachtung hauptsächlich der Wirkungen der Mittelverausgabung beschränkt; die Effekte der Mittelbeschaffung bleiben nahe$\mathrm{zu}$ unberücksichtigt. Es geht zweitens vor allem darum, daB durch eine Transformationsausgabenerhöhung die private Nachfrage nicht so angeregt wird, wie Keynesianer vermuten und weniger um eine Verdrängung von ansonsten getätigten privaten Ausgaben. Gegenstand der folgenden Betrachtung sind also pulling-in-Teileffekte.

\section{B. Zum direkten Staatsausgabeneffekt}

Transformationsausgaben werden üblicherweise, so auch im Grundmodell, als Bestandteil der von Staat und Privaten auf dem Gütermarkt entfalteten Nachfrage angesehen. Zusätzliche Transformationsausgaben erhöhen mithin genau um ihren Betrag die gesamtwirtschaftliche Güternachfrage. Bei Transferzahlungen ist dies anders; durch sie wird kein direkter staatsausgabeneffekt bewirkt. Folgende zwei Gründe sprechen allerdings dafür, daB dies eine Eigen- 
schaft nicht nur der Transferzahlungen ist, sondern auch eine Eigenschaft der Transformationsausgaben sein kann.

Erstens: Die Transformationsausgaben setzen sich aus Sachausgaben und Personalausgaben zusammen. Personalausgaben sind die Ausgaben zur Entlohnung der im öffentlichen Dienst Beschäftigten. Tätigt der staat Personalausgaben, tritt er nicht auf dem Gütermarkt, sondern auf dem Arbeitsmarkt als Nachfrager auf. Zusätzliche Personalausgaben führen, genau wie Transferzahlungen, zu keiner unmittelbaren Erhöhung der gesamtwirtschaftlichen Güternachfrage. Eine Transformationsausgabenerhöhung hat also nur insoweit einen direkten staatsausgabeneffekt, wie die sachausgaben zunehmen ${ }^{1}$ !

Zweitens: Da der Staat seine Transformationsausgaben kreditär finanziert, muB er einen Teil der vereinnahmten Geldmittel für die zinszahlungen reservieren. Diese Gelder stehen nicht zur Entfaltung von Güternachfrage zur Verfügung. Insoweit wird auch hier kein direkter staatsausgabeneffekt bewirkt.

Nimmt beispielsweise der Staat einen Kredit in Höhe von $10 \mathrm{Mrd}$. DM zu Beginn einer Haushaltsperiode auf, so werden bei einem Zinssatz von 8 \% $\mathrm{zu}$ deren Ende $800 \mathrm{Mill}$. DM an Zinszahlungen fällig. Dramatischer ist das Ergebnis in mittelfristiger Betrachtung. Bei einer dauerhaften Zunahme der Transformationsausgaben und konstant bleibendem Steueraufkommen steigt von Haushaltsperiode zu Haushaltsperiode der Bestand an Staatspapieren in den Portefeuilles der Privaten um $10 \mathrm{Mrd}$. DM an. In der fünften Periode belaufen sich bei den angenommenen Zahlenwerten die zinszahlungen schon auf $4 \mathrm{Mrd}$ DM. Von den $10 \mathrm{Mrd}$. DM zusätzlichen Staatsausgaben haben nur 6 Mrd. DM einen direkten Staats-

1) Vgl. Svindland, E., Staatsausgaben und ihre Finanzierung. Einige elementare Bemerkungen zu den Grundlagen der Crowding-out-Analyse, in: Vierteljahreshefte zur Wirtschaftsforschung, 1980, s. $163 \mathrm{f}$. 
ausgabeneffekt ${ }^{11}$.

Fazit: Kreditär finanzierte Staatsausgaben bewirken auf mittlere Frist ein "internes" crowding-out. "Denn das, was der staat zusätzlich an Gütern und Diensten kaufen kann, wird allmählich kleiner, weil er nachfragewirksame Ausgaben durch höhere $\mathrm{zinszahlungen} \mathrm{er-}$ setzen muß, die für die zusätzliche staatsverschuldung anfallen" 2 ?

C. Zum einkommensinduzierten Konsumeffekt

1. Permanentes Einkommen als Bestimmungsgrund der privaten Konsumausgaben

Eine Transformationsausgabenmehrung steigert im Grundmodell das Niveau der gesamtwirtschaftlichen Ausgaben nicht nur additiv in Höhe ihres Betrages, sondern durch den einkommensinduzierten Konsumeffekt auch multiplikativ um ein Mehrfaches dieses Betrages. Das AusmaB des Multiplikatoreffekts hängt von der Neigung der Privaten $a b$, zusätzliches, aktuelles Einkommen zu verbrauchen. Keynesianer gehen davon aus, daB diese Neigung vergleichsweise groB ist.

1) Derartige Modellrechnungen gehen auf Domar zurück. Domar hat gezeigt, daB für den Fall einer dauerhaften, jährlichen Kreditaufnahme in fester Relation zum Sozialprodukt und einer konstanten Wachstumsrate des Sozialprodukts langfristig nur dann die anfallenden zinszahlungen die jährliche Kreditaufnahme übersteigen, wenn der Zinssatz für die Staatsschulden höher als die wachstumsrate des Sozialprodukts ist. Andernfalls ergibt sich dauerhaft ein Haushaltsspielraum bzw. bei Verwendung dieses Haushaltsspielraums zur Tätigung von Transformationsausgaben auf Dauer ein direkter staatsausgabeneffekt. Vgl. z.B. Kitterer, W., Uber die Grenzen der Staatsverschuldung, Manuskript, 1980, S. 4 ff.

2) Sachverständigenrat zur Begutachtung der gesamtwirtschaftlichen Entwicklung, Jahresgutachten $1978 / 79$, a.a.0., Ziff. 483 . 
Die keynesianische Einschätzung des privaten Konsumverhaltens ist von Milton Friedman und anderen zurückgewiesen worden ${ }^{1}$ ! Nach Friedman spalten die Privaten das aktuelle Einkommen gedanklich in zwei Teile auf: in einen transitorischen, d.h. später wieder wegfallenden und in einen permanenten, d.h. dauerhaften Teil. Die Neigung, aus transitorischem Einkommen $\mathrm{zu}$ verbrauchen ist im Gegensatz zur Neigung, aus permanentem Einkommen $z u$ konsumieren nach Ansicht Friedmans gering.

Mit dem von Keynesianern vermuteten AusmaB des einkommensinduzierten Konsumeffekts ist demnach nur dann zu rechnen, wenn sich das permanente Einkommen erhöht. Dies ist der Fall, wenn Staatsausgaben auf Dauer zunehmen.

Dagegen versickert ein zeitlich befristeter staatsausgabenimpuls schon in einem frühen stadium des erhofften Expansionsprozesses. Wegen der zeitlichen Limitierung steigt nämlich weniger das permanente, sondern hauptsächlich das transitorische Einkommen. Da die Neigung, aus transitorischem Einkommen zu konsumieren, gering ist, fält der einkommensinduzierte Konsumeffekt nur sehr schwach aus; der direkte staatsausgabeneffekt pflanzt sich kaum auf die Konsumausgaben fort.

Fazit: Mit der permanenten Einkommenshypothese "ist ... der ganze Wirkungsmechanismus, auf den die fiskalistische

1) Für einen Uberblick vgl. Landmann, 0., Keynes in der heutigen Wirtschaftstheorie, a.a.0., s. $182 \mathrm{ff}$. sowie Tobin, J., Buiter, W., Fiscal and Monetary Policies, Capital Formation, and Economic Activity, in: Fürstenber, G.M. von (Hrsg.). The Government and Capital. Formation, Cambridge, 1980 , s. $81 \mathrm{ff}$. 
Stabilisierungspolitik baut, in Frage gestellt"1), soweit die stabilisierungspolitischen MaBnahmen zeitl.ich begrenzt sind. Denn auf zeitlich befristete finanzwirtschaftliche Impulse reagieren die Privaten nur sehr träge mit einer Anpassung ihrer Konsumausgaben. Der einkommensinduzierte Konsumeffekt hat dann nur einen geringen Wert.

2. Offentliche Güter als Bestimmungsgrund der privaten Ausgaben

a) Die Staatsausgaben im Güterkreislauf

Staatliche Aktivitäten, die sich im Grundmodell in einer Erhöhung der Transformationsausgaben niederschlagen, wirken sich im volkswirtschaftlichen Güterkreislauf in zwei Richtungen aus ${ }^{2}$ ! Zum einen tätigt der staat Ausgaben; er tritt damit auf dem Gütermarkt als Nachfrager auf und entzieht dem privaten Sektor Ressourcen (Inputeffekt). Mit den erworbenen Gütern und Diensten werden zum anderen vom Staat öffentliche Leistungen erstellt und meist ohne spezielles Entgelt an die Privaten abgegeben; damit bietet der staat auf dem Gütermarkt an und führt dem privaten Sektor Ressourcen zu (Outputeffekt).

Der EinfluB des Inputeffekts zusätzlicher Transformationsausgaben auf die privaten Ausgaben ist im Grundmodell durch den direkten staatsausgaben- und den einkommensinduzierten Konsumeffekt erfaBt. Keine Berücksichtigung

1) Landmann, 0., Keynes in der heutigen Wirtschaftstheorie, a.a.O., S. 183.

2) Vgl. z.B. Peffekoven, R., రffentliche Finanzen, in: Vahlens Kompendium der Wirtschaftstheorie und Wirtschaftspolitik, München, 1980 , S. 441. 
dagegen findet der Outputeffekt ${ }^{1)}$. Dieser kann durchaus kontraproduktiv sein und bildet das Untersuchungsfeld der folgenden Uberlegungen.

Für das Räsonnement ist eine Differenzierung der Transformationsausgaben, die an der Art des bewirkten Outputeffekts anknüpft, sinnvoll (vgl. Ubersicht 10). Transformationsausgaben unterscheiden sich von Transferzahlungen dadurch, daB sie mit einem Entzug von Ressourcen aus dem privaten Bereich verbunden sind (Entzugskriterium). Die erworbenen Güter und Dienste versetzen den Staat in die Lage, den Privaten öffentliche Güter anzubieten. In Abhängigkeit vom Zeitpunkt, zu dem die Privaten von der öffentlichen Leistungsabgabe profitieren, können Transformationsausgaben mit dem Attribut konsumtiv oder investiv versehen werden (Periodisierungskriterium) 2 ). Investitionsausgaben werden im Hinblick auf zukünftige Erträge, Konsumausgaben im Hinblick auf gegenwärtige Erträge getätigt. Gleichgültig, ob die durch zusätzliche Transformationsausgaben bewirkten Erträge bei den Privaten schon in der Gegenwart anfallen oder erst in der Zukunft, können sie entweder als Vorleistungen der Produktion von Gutern in den privaten Unternehmen dienen oder als Endleistungen dem Konsum in den privaten Haus-

1) "In Analogie zur betriebswirtschaftlichen Untersuchung könnte man auch sagen, die herkömnliche Fiskaltheorie beschränkt sich auf die'Finanzierung 'und den'Einkauf' und vernachlässigt die'Produktion'und den (meist kostenlosen) 'Verkauf'öffentlicher Leistungen". Meister, J., Konjunkturelle Effekte des Budgets - eine allokative Betrachtung, Referat, gehalten auf dem KongreB "Junge Wissenschaft und Wirtschaft" der Hanns Martin Schleyer-Stiftung, Innsbruck, November 1980 , S. 1 .

2) Vgl. Wissenschaftlicher Beirat beim Bundesministerium der Finanzen, Zum Begriff der öffentlichen Investitionen, Bonn 1980, S. $39 \mathrm{ff}$. 
Ubersicht 10: Die Staatsausgaben im Güterkreislauf



haltungen (Empfangskriterium) ${ }^{1}$ !

R. Frey unterscheidet dementsprechend bei den öffentlichen Infrastrukturinvestitionen "Produktivinvesti-

1) Zur Lokalisierung des Empfängerkreises von Staatsleistungen vgl. Leipert, G., Staatskonsum, staatliche Investition und die Produktivität staatlichen Handelns, in: Wirtschaftsdienst, 1979, s. $149 \mathrm{ff}$. 
tionen" von "Konsumtivinvestitionen"1) Analog kann man den öffentlichen Konsum in "Produktivkonsum" und "Konsumtivkonsum" differenzieren")

b) Befriedigung bisher privat gedeckter Güternachfrage durch öffentliche Leistungen

Weitet der Staat durch zusätzliche Transformationsausgaben seine Leistungen in Bereichen aus, die zuvor privaten Produzenten rentable Produktionsmöglichkeiten eröffnet haben, so tritt eine Verdrängung der privaten Produzenten und - was hier wichtig ist - deren Ausgaben ein ${ }^{3 !}$. Denn da die staatlichen Leistungen meist ohne spezielles Entgelt abgegeben werden, ist der private Produzent jetzt nicht mehr konkurrenzfähig und muB seine produktion einstellen.

1) Vgl. Frey, R.L., Infrastruktur. Grundlagen, der Planung öffentlicher Investitionen, Tübingen, $1972^{2}$, S. 18 .

2) So gesehen sind die Ausgaben für den Bau einer AutostraBe eine öffentliche Investition, und das Gehalt für den Verkehrspolizisten, der auf dieser StraBe den Autoverkehr regelt, ist öffentlicher Konsum. Fährt ein LKW auf dieser StraBe, werden also öffentliche Leistungen als Vorleistungen in der privaten produktion eingesetzt, handelt es sich um eine Produktivinvestition und um Produktivkonsum. Wird die Straße dagegen von einem Privat-PKW benutzt, sind die (anteiligen) öffentlichen Ausgaben für den Bau der StraBe und den Verkehrspolizisten eine Konsumtivinvestition und Konsumtivkonsum.

3) "There are several levels at which crowding-out has been alleged to occure. The most obvious is the possibility that government will engage in productive activities which would otherwise be provided by the private sector, so that public spending would simply supplant private investment". Blinder, A.S., Solow, R.M., Does Fiscal Policy Matter?, a.a.o., S. 320. 
Die staatliche Leistung kann von den Privaten entweder als Vorleistung für die Produktion oder als Endleistung für den Konsum verwendet werden. Dem erstgenannten Fall ist in juingster zeit in der wachstumstheoretischen Literatur viel Aufmerksamkeit geschenkt worden. Unentgeltliche staatliche Vorleistungen wirken sich mindernd auf die privaten Kosten der Produktion aus und stimulieren damit die private Investitionstätigkeit. Insoweit die Staatsleistungen Endleistungen für den Konsum darstellen, werden für die Konsumenten erst einmal ansonsten zu tätigende Ausgaben überflüssig. Wenn daraufhin lediglich die Konsumstruktur variiert, tritt kein crowdingout auf; anders liegen die Dinge, wenn sich das Konsumniveau ändert, d.h. weniger verbraucht wird. Dieser Fall wird in der Literatur als "Bailey-These" diskutiert.

c) Die Bailey-These

aa) Die Baileysche Konsumfunktion

Bailey knüpft an die im Grundmodell verwendete Keynessche Konsumfunktion an und modifiziert diese in zweifacher Weise") Zum einen geht er davon aus, daB die pri-

1) Vgl. Bailey, M.J., National Income and the Price Level, New York u.a., 1962, S. 71 ff.; Peacock, A.T., The Multiplier and the Valuation of Government Expenditures, in: Finanzarchiv, 1972, S. $418 \mathrm{ff}$ : : Peacock, A.T., Shaw, G.K., Is Fiscal Policy Dead?, in: Banca Nazionale del Lavoro Quarterly Review, June 1978, S. 114; die Bailey-These wird ausfuhrlich behandelt von Zundel, M., EinfluB der öffentlichen Güter auf den privaten Konsum, Bochumer Dissertation, 1978, hier insbesondere S. $39 \mathrm{ff.;} \mathrm{fur} \mathrm{ähnlich} \mathrm{intendierte} \mathrm{Uber-}$ legungen vgl. Meister, J., Budget und Konjunktur. Eine allokative ProzeBanalyse, Göttingen, 1981. 
vaten Haushalte die durch Transformationsausgaben bewirkten öffentlichen Leistungen als Naturaleinkommen ansehen und dieses gleichwertig neben ihr Geldeinkommen stellen. Das Gesamteinkommen ergibt sich dann aus der Summe von verfügbarem Geldeinkommen $(Y-T)$ und den Transformationsausgaben $(\bar{G})$. Zum zweiten unterstellt Bailey, daB die privaten Haushalte die vom staat getätigten konsumtiven Transformationsausgaben und ihre eigenen Konsumausgaben als gleichwertig im Hinblick auf die Befriedigung ihrer Konsumbedürfnisse ansehen. Der Gesamtkonsum der privaten Haushalte setzt sich folglich aus den privaten Konsumausgaben $(C)$ und den öffentlichen Konsumausgaben $\left(\bar{G}^{C}\right)$ zusammen. Er ist nach MaBgabe der marginalen Konsumquote (c) eine Funktion des Gesamteinkommens:

$$
(2-3-1) \quad C+\bar{G}^{C}=c \cdot[(Y-T)+\bar{G}] .
$$

Die privaten Konsumausgaben bestimmen sich also durch

$$
(2-3-2) \quad C=C \cdot[(Y-T)+\bar{G}]-\bar{G}^{C}
$$

bzw.:

$$
(2-3-3) \quad C=C \cdot\left[(Y-T)+\bar{G}^{I}\right]-(1-C) \cdot \bar{G}^{C} \text {. }
$$

bb) Ausgabenmultiplikatoren

Wird Gleichung (2-3-3) partiell nach den staatlichen Konsumausgaben $\left(\mathrm{d} \overline{\mathrm{G}}^{\mathrm{C}}\right)$ differenziert, erhält man als Veränderung der privaten Konsumausgaben (dC):

$$
(2-3-4) \quad \mathrm{dC}=-(1-\mathrm{c}) \cdot \mathrm{d} \overline{\mathrm{G}}^{\mathrm{C}}
$$

Unter der Baileyschen Annahme werden mithin private 
Konsumausgaben durch öffentliche nicht angeregt, sondern verdrängt. Dies hat darin seine Ursache ${ }^{1}$ ! da $B$ konsumtive staatliche Zusatzausgaben einerseits das Gesamteinkommen, andererseits aber auch den Gesamtkonsum der Privaten um ihren Betrag erhöhen. Zusätzliches Gesamteinkommen wünschen die privaten Haushalte jedoch nur nach Maßgabe ihrer marginalen Konsumquote $z u$ Konsumzwecken $z u$ verwenden. Da der eine Teil des Gesamtkonsums, nämlich der staatliche Konsum, für die privaten Haushalte nicht beeinfluBbar ist, verbleibt ihnen nur eine Verringerung des anderen Teils - der private Konsum - um das tatsächliche an das gewünschte Konsumniveau anzupassen.

Demzufolge hat auch der Privatausgabenmultiplikator im Vergleich zu einem Referenzmultiplikator des Grundmodells einen geringeren Wert. Dort beträgt der Privatausgabenmultiplikator einer Erhöhung der (konsumtiven) Transformationsausgaben, wenn zur Vereinfachung $d i=d T=0$ unterstellt wird:

$$
(1-4-33) \frac{\mathrm{d}(\mathrm{C}+\mathrm{I})}{\mathrm{d} \overline{\mathrm{G}}^{\mathrm{C}}}=\frac{1}{1-\mathrm{C}}-1=\frac{\mathrm{C}}{1-\mathrm{C}} \quad \text { (Grundmodell). }
$$

Wird statt der Keynesschen Konsumfunktion (1-4-15) die Baileysche (2-3-3) verwendet, und wird ebenfalls davon ausgegangen, $\mathrm{da} B \mathrm{di}=\mathrm{dT}=0$ ist, lautet der Privatausgabenmultiplikator:

$$
(2-3-5) \frac{\mathrm{d}(\mathrm{C}+\mathrm{I})}{\mathrm{d} \overline{\mathrm{G}}^{\mathrm{C}}}=\frac{2 \mathrm{c}-1}{1-\mathrm{C}} \quad \text { (Bailey-Fall). }
$$

Da $c<1$ ist, hat er im Vergleich zum Referenzmultiplikator

1) Vgl. Zundel, M., EinfluB der öffentlichen Güter auf den privaten Konsum, a.a.O., S. $40 \mathrm{f}$. 
(1-4-33) einen kleineren Wert; dies ist auf die durch die staatliche Leistungsabgabe induzierte Substitution von privaten Konsumausgaben $(\mathrm{dC})$ durch öffentlichen Konsum $\left(\mathrm{dG} \bar{G}^{C}\right)$ zurückzuführen.

\section{cc) Einschränkungen}

Folgende drei. tberlegungen schränken allerdings die Bedeutung der Bailey-These zur Begrundung eines crowdingout ein.

Erstens: GemäB dem Empfangskriterium können die mit konsumtiven Transformationsausgaben erstellten öffentlichen Leistungen den Produzenten oder den Konsumenten zugute kommen. Bailey ignoriert offensichtlich die erstgenannte Möglichkeit; denn seine These trifft allein für konsumtive Transformationsausgaben $z u$, deren Erträge in den privaten Haushalten anfallen. Damit wird der Gültigkeitsbereich der Bailey-These eingeschränkt ${ }^{1}$ !

Zweitens: DaB der Privatausgabenmultiplikator im BaileyFall einen geringeren wert annimmt als der Referenzmultiplikator, läßt sich auch damit begründen, im Grundmodell werde mit der Annahme gearbeitet, die privaten Haushalte würden einer Fiskalillusion unterliegen. Fiskalillusion in diesem Sinne kann darin ihren Ursprung haben, daB einmal die Privaten übersehen, daB die vom staat beanspruchten Ressourcen in Form unentgeltlicher öffentlicher Leistungen wieder an sie zurückflieBen oder, daB sie zum anderen den zufluB zwar wahrnehmen, ihn aber mit einem Wert nahe von Null versehen. Bei Existenz dieser

1) Vgl. Zundel, M., EinfluB der öffentlichen Güter auf den privaten Konsum, a.a.0., S. $64 \mathrm{ff}$. 
Fiskalillusion verschwinden in $(2-2-3)$ die Bestimmungsgründe $\bar{G}^{I}$ und $\bar{G}^{C}$ der privaten Konsumausgaben, und die Baileysche Konsumfunktion geht in die Keynessche uber. Bailey dagegen unterstellt Freiheit von Fiskalillusion ")

Drittens: Wenn der Staat anstatt konsumtiver zusätzlich investive Transformationsausgaben $\left(\mathrm{d} \overline{\mathrm{G}}^{\mathrm{I}}\right)$ tätigt, nimmt der Baileysche Privatausgabenmultiplikator einen wert an, welcher größer ist als der des Referenzmultiplikators ${ }^{2}$ ?

$$
(2-3-6-) \quad \frac{d(C+I)}{d \bar{G}^{I}}=\frac{2 c}{1-c} .
$$

Denn eine Erhöhung der investiven Transformationsausgaben vermehrt gemäB Bailey nur das Naturaleinkommen der privaten Haushalte, nicht aber die Menge der den privaten Haushalten zur Verfügung stehenden öffentlichen Konsumgütern.Die private Konsumnachfrage wird also durch die zusätzlichen Staatsleistungen nicht gedämpft, sondern angeregt.

Allerdings dürfen öffentliche Investitionsausgaben nicht schon in der Periode ihrer Verausgabung als Naturaleinkommen angesetzt werden ${ }^{3}$. Investive Trans-

1) Bailey geht in seinen Ausführungen von einer Bewertung zu Kosten aus, d.h. von einer Bewertung mit den Transformationsausgaben, die zur Beschaffung der Ressourcen nötig sind, welche zur Erstellung der öffentlichen Leistungen eingesetzt werden. Vgl. hierzu ausfürlich: ebenda, S. $68 \mathrm{ff}$.

2) Vgl. Bailey, M.J., National Income and Price Level, a.a.0., S. 72 ff.

3) Vgl. hierzu auch Zundel, M., EinfluB der öffentlichen Güter auf den privaten Konsum, a.a.0., S. 60 ff. 
formationsausgaben sind nämlich dadurch gekennzeichnet, daB sie erst in der Zukunft einen Ertrag bringen bzw. daB das Naturaleinkommen erst zukünftig den privaten Haushalten zufließt. Dann entfällt auch die Erhöhung des Naturaleinkommens, und der Privatausgabenmultiplikator einer investiven Transformationsausgabenausdehnung reduziert sich auf den Referenzmultiplikator.

d) Die David/Scadding-These

aa) Annahmen

David und Scadding kommen $z$ dem Ergebnis, daß die Privaten ihre Ausgaben immer gegenläufig zu denen des Staates variieren und deshalb das Gesamtausgabenniveau und damit das Einkommen von einer Transformationsausgabenerhöhung nicht tangiert wird. Folglich entfällt der einkommensinduzierte Konsumeffekt.

Ausgangspunkt ihrer tberlegungen" ist das "Denison Gesetz". Demnach ist die Aufteilung der insgesamt verfügbaren Ressourcen auf investive und konsumtive Verwendungen über die Zeit konstant. Die Privaten streben mithin eine feste Relation zwischen den in der Volkswirtschaft zugunsten gegenwärtiger und den zugunsten

1) Vgl. David, P.A., Scadding, J.L., Private Savings: Ultrarationality, Aggregation, and "Denison's Law", a.a.0., S. $225 \mathrm{ff,;}$ einen knappen Uberblick geben Carlson, K.M., Spencer, R.W., Crowding Out and its Critics, a.a.0., S. 7 f.; Dieckheuer, G., Der Crowding-out-Effekt - zum gegenwärtigen Stand von Theorie und Empirie, a.a.O., S. 129 f. sowie Fürstenberg, G.M. von, Public versus Private Spending: The Long-Term Consequences of Direct Crowding-Out, in: derselbe (Hrsg.), The Government and Capital Formation, Cambridge, Massachusetts, 1980, s. $245 \mathrm{ff}$. 
zukünftiger Erträge eingesetzten Ressourcen an.

Wer über die Ressourcen disponiert - Private oder der Staat - ist den Privaten gleichgültig. Die Ausgaben werden allein danach klassifiziert, wann die mit ihnen verbundenen Erträge anfallen - in der Gegenwart oder in der Zukunft. Folglich sind private und staatliche Konsumausgaben einerseits und private und staatliche Investitionsausgaben andererseits aus sicht der Privaten vollkommene substitute.

Zudem unterstellen David und Scadding, die Privaten würden von der Finanzierungsform staatlicher Transformationsausgaben auf den zeitpunkt der damit bewirkten staatsleistungen schließen: Kreditär finanzierte Transformationsausgaben werden als investive, steuerfinanzierte als konsumtive identifiziert.

Zusammengenommen: David und Scadding gehen davon aus, daB erstens die Privaten eine feste Relation zwischen (privaten und öffentlichen) Konsumausgaben und (privaten und öffentlichen) Investitionsausgaben wünschen, daB sie zweitens gegenüber den jeweiligen öffentlichen und privaten Anteilen daran indifferent sind und daB drittens die Privaten von der Finanzierungsform auf die Art der Transformationsausgaben schlieBen. 
bb) Stabilisierungspolitische Konsequenz: vollständiges, direktes crowding-out

Daraus folgern David und Scadding für die stabilisierungspolitische Effizienz einer finanzpolitischen Intervention: "There is no way that changes in the level of public expenditures can affect aggregate demand and output (or prices); fiscal policy would be useless for stabilization purposes"1) Denn zusätzliche, kreditär finanzierte Transformationsausgaben werden von den Privaten als Investitionen gewertet, die in ihrem Umfang private Investitionen überflüssig machen. Eine mit Steuern finanzierte Transformationsausgabenerhöhung wird von den Privaten als Substitut für ansonsten selbst zu tätigende Konsumausgaben angesehen und führt um ihren Betrag zu einer Einschränkung des privaten Konsums.

In das Grundmodell lassen sich die tberlegungen von David und scadding dadurch einbauen, daB das Steueraufkommen $(T)$ in der Konsumfunktion und die Kreditmarktverschuldung des Staates $\left(B-B_{-1}\right)$ in der Investitionsfunktion mit jeweils einem Koeffizienten von -1 als Argument berücksichtigt werden ${ }^{2}$ !

$(2-3-7) \quad C=C(Y-T)-T$,
$(2-3-8) \quad I=I(i)-\left(B-\bar{B}_{-1}\right)$.

1) David, P.A., Scadding, J.L., Private Savings: Ultrarationality, Aggregation, and "Denison's Law", a.a.O., S. 245 .

2) Vgl. Butkiewicz, J.L., Some Macroeconomic Effects of Government Debt, a.a.0., S. $29 \mathrm{ff}$. 
Steuerfinanzierte Transformationsausgaben und private Konsumausgaben sowie kreditfinanzierte Transformationsausgaben und private Investitionsausgaben sind völlige Substitute. Steigen die Staatsausgaben, werden die Privatausgaben um diesen Betrag eingeschränkt. Es findet ein vollständiges, direktes crowding-out statt.

Die IS- und die LM-Gleichung lauten dann

$$
(2-3-9) \quad Y-C(Y-T)+T-I(i)+\left(B-\bar{B}_{-1}\right)-\bar{G}=0 \text {, }
$$

bzw. wegen

$$
\begin{aligned}
& (1-4-24) \quad \bar{G}-T=B-\bar{B}-1 \quad: \\
& (2-3-10) \quad Y-C(Y-T)-I(i)=0
\end{aligned}
$$

und

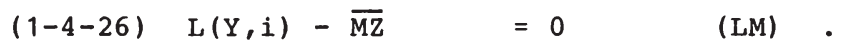

Die Güter- und Geldmarktgleichgewichtsbedingungen enthalten keine finanzwirtschaftlichen Parameter mehr. Eine Erhöhung der Transformationsausgaben hat mithin keinen EinfluB auf das Niveau der Gesamtausgaben, d.h. durch eine Transformationsausgabenerhöhung werden um den Betrag der staatlichen Zusatzausgaben private Ausgaben verdrängt.

cc) Kritische würdigung

Bei einer Beurteilung der Brauchbarkeit der David/ Scadding-These zur Begründung eines vollständigen, direkten crowding-out bei Unterbeschäftigung ist folgendes zu bedenken. 
Erstens: Ein objektbezogener Deckungsgrundsatz dergestalt, daB der Staat investive Ausgaben mit Krediten und konsumtive mit steuern $z u$ finanzieren hat, existiert in der Bundesrepublik Deutschland seit der Reform des Haushaltsrechts Ende der sechziger Jahre nicht mehr. Es ist laut Grundgesetz, Artikel 109 (2) vielmehr bei der Haushaltswirtschaft "den Erfordernissen des gesamtwirtschaftlichen Gleichgewichts Rechnung zu tragen": bei Unterbeschäftigung ist diejenige Finanzierungsform zu wählen, mit der wenig Verdrängungseffekte verbunden sind, gleichgültig, ob mit den finanziellen Mitteln Investitions- oder Konsumausgaben getätigt werden.

Hieraus folgt: Auch wenn die Hypothese von David und Scadding, die Privaten wïrden von der Finanzierungsform auf die Art der Transformationsausgaben schlieBen, richtig wäre, so geht dann die SchluBfolgerung der Privaten an der Wirklichkeit vorbei. Allerdings spielt ihr FehlschluB für den Umfang der verdrängten Privatausgaben keine Rolle. Denn die Privaten schränken gemäB David und Scadding ihre Ausgaben um den Betrag der zusätzlichen Transformationsausgaben ein - unabhängig davon, ob die Zusatzausgaben des Staates kreditär oder mit steuern finanziert werden. Was allein davon betroffen wird, ist die Art der von den Privaten vermindert getätigten Ausgaben. Bei einer Steuerfinanzierung schränken die Privaten die konsumtiven Ausgaben, bei einer Kreditfinanzierung die investiven Ausgaben ein.

Zweitens: Die Anreizmechanismen, welche einzelne Investoren dazu bewegen, bei einer Zunahme der Staatsverschuldung ihre Investitionspläne $z u$ vertagen und welche einzelne Konsumenten dazu veranlassen, bei einer steuererhöhung ihren Verbrauch einzuschränken, bleiben bei David und 
Scadding im Dunkeln. Indirekte Mechanismen scheiden als Erklärung eines direkten crowding-out aus. Eine unmittelbare Substitution von privaten durch staatliche Ausgaben à la David und Scadding wäre denkbar, wenn nur die Konsumenten der Steuerpflicht unterlägen und der Staat sich allein bei den Investoren verschulden würde. Dann hätten die einzelnen Wirtschaftssubjekte die nötigen Informationen, um ihre einzelwirtschaftlichen Pläne mit dem staatlichen Haushaltsplan im Hinblick auf die gesamtwirtschaftlich gewünschte Relation zwischen investiven und konsumtiven Ausgaben zu koordinieren.

Drittens: Auch wenn mit David und Scadding angenommen wird, die Privaten hätten bei ihren Ausgabendispositionen tatsächlich eine gewünschte gesamtwirtschaftliche Relation zwischen investiven und konsumtiven Ausgaben als Richtschnur vor Augen, folgt daraus keineswegs zwingend, daB sie weniger Güter nachfragen, wenn der Staat einen gröBeren Teil des Produktionspotentials absorbiert. Die gesamtwirtschaftliche Güternachfrage ist nämlich nicht durch das aktuelle, sondern durch das bei Vollbeschäftigung mögliche Sozialprodukt begrenzt.

Im Unterbeschäftigungsfall, in dem nach Ansicht von David und Scadding ebenso wie bei Vollbeschäftigung mit einem vollständigen, direkten crowding-out zu rechnen ist ${ }^{1)}$, brauchen mithin die Privaten keineswegs ihre Ansprüche kompensierend zurückzustellen, um dadurch eine durch die staatliche Intervention bewirkte Abweichung der gesamtwirtschaftlichen Relation zwischen investiven und konsumtiven Ausgaben von dem gewünschten Wert zu neutralisieren. Die gewünschte Relation kann sich ebenso einstellen, wenn die Privaten ihre Güternachfrage komplementär

1) Vgl. David, P.A., Scadding, J.L., Private Savings: Ultrarationality, Aggregation, and "Denison's Law", a.a.0., S. 243 ff. 
zu den staatlichen Zusatzausgaben ausdehnen, also bei zusätzlichen konsumtiven Transformationsausgaben mehr investieren und bei zusätzlichen investiven Transformationsausgaben mehr konsumieren.

D. Zum Akzelerationseffekt

1. Investitionsattentismus: angebotsseitige und nachfrageseitige Ursachen

Das Kapitel abschließend sei noch die These der Keynesianer überprüft, daB infolge einer Erhöhung der Transformationsausgaben mit einem Akzelerationseffekt $z u$ rechnen ist: Die zusätzliche Güternachfrage des Staates und die dadurch induzierte Güternachfrage der privaten Haushalte steigern den Auslastungsgrad des Sachkapitals und regen - so die Vermutung der Keynesianer - die Unternehmer an, ihren Bestand an Sachkapital auszuweiten.

Die Ursachen, weshalb Unternehmer bei ihren Investitionsplänen Zurückhaltung üben, können mit dem Sachverständigenrat in nachfrageseitige und angebotsseitige separiert werden ${ }^{1)}$. Dabei ist das Unterscheidungskriterium für die Gründe, warum das sachliche Produktionspotential nicht erweitert

1) Vgl. hierzu die Gutachten des Sachverständigenrates zur Begutachtung der gesamtwirtschaftlichen Entwicklung seit 1976, insbesondere: Sachverständigenrat zur Begutachtung der gesamtwirtschaftlichen Entwicklung, Jahresgutachten 1976/77, a.a.O., Ziff. 272 ff.; derselbe, Mehr Wachstum, mehr Beschäftigung, Jahresgutachten 1977/78, a.a.0., Ziff. 236 ff.; derselbe, Jahresgutachten 1978/79, a.a.0., Ziff. 266 ff.; derselbe, Jahresgutachten 1980/81, a.a.0., Ziff. 495 ff.; vgl, auch Sievert, O., Die Steuerbarkeit der Konjunktur durch den Staat, a.a.o., S. 810 ff.; derselbe, Arbeitslosigkeit und Lohnhöhe, a.a.0., s. 13 ff.; derselbe, Position des Sachverständigenrates, a.a.O., S. $18 \mathrm{ff}$. sowie Brenner, G., Lipp, E.M., Beschäftigungspolitische Verantwortung der Finanzpolitik. Probleme bei ambivalenter Ursachendiagnose, a.a.O., S. 1 ff.;Lipp, E.M., Staatlicher Handlungsbedarf bei anhaltender Arbeitslosigkeit - Bemerkungen zu einer anhaltenden Kontroverse,a.a.0., S. $155 \mathrm{ff}$. 
wird, genau das gleiche wie für die Gründe, warum das vorhandene Produktionspotential nicht in vollem AusmaB genutzt wird, also Unterbeschäftigung besteht: Nehmen die Störimpulse ihren Ausgang bel der Verteilung des Ergebnisses der Produktion, also auf der Angebotsseite, oder bel der Verwendung der Produktion, also auf der Nachfrageseite? ${ }^{1)}$

Verhalten sich Unternehmer als Gewinnmaximierer, vermehren sie ihr Sachkapital immer dann, wenn sich mit der Investition für sie rentabel erscheinende produktionschancen eröffnen. Die für die Investitionsphase erwarteten Verkaufserlöse müssen den Unternehmern reichen, die fremden Ansprüche an das Produktionsergebnis (von Arbeitnehmern, Fremdkapitalgebern sowie des Staates) und auch die eigenen (zur Abdeckung des Unternehmerlohnes, der Verzinsung des Eigenkapitaleinsatzes und eine Unsicherheitsprämie) zu befriedigen. Andernfalls lohnt es für die Unternehmer nicht $z u$ investieren $^{2)}$.

Durch dieses Gewinnkalkül wird die Bedeutung des "mechanistischen" Akzelerationseffekts (zusätzliche Güternachfrage $\longrightarrow$ zusätzliche private Investitionen) eingeschränkt. Infolge einer Nachfrageexpansion kommt es nur dann zu einem Akzelerationseffekt, wenn bislang nicht lohnende Produktionschancen nun rentabel werden. Voraussetzung eines solchen Vorzeichenwechsels bei Investitionsrechnungen ist, daB die Gründe für das vorherige Unterlassen der Investitionen auf der Nachfrageseite und nicht auf der Angebotsseite des Gütermarktes liegen.

1) Vgl. Seite $41 \mathrm{ff}$. oben.

2) "Niemand kann sich ja der schlichten Anschauung erwehren, daß die Menge der Betriebe und Betriebsabteilungen, die ... neu gegründet werden, letztlich davon abhängt, mit welchen Ansprüchen an das Produktionsergebnis die in einem Betrieb Zusammenwirkenden antreten". Sievert, O., Position des Sachverständigenrates, a.a.0., s. 22 . 
Investitionshemmende störimpulse haben ihre Wurzel auf der Güternachfrageseite, wenn die Lohn-, Zins- und $\mathrm{Ab}-$ gabenansprüche an das Produktionsergebnis mit denen des Unternehmers vereinbar wăren, sofern nur höhere Verkaufserlöse erzielt werden können - eine zunahme der Erlöse jedoch durch fehlende Guternachfrage verhindert wird. Es wurde mehr investiert, wenn von staat und Privaten mehr Güter nachgefragt wirden.

Wird dagegen das Gewinnkalkul der Investoren von angebotsseitigen Störimpulsen dominiert, investieren die Unternehmer deswegen nicht, weil die Summe der Ansprüche an das Produktionsergebnis selbst bei Vollauslastung des Sachkapitalzuwachses das Produktionsergebnis übersteigt. Den Unternehmern wird - anders gewendet - nur ein Anteil am Produktionsergebnis zugestanden, der in einer Größenordnung liegt, bei dem die Alternative, die Investition zu unterlassen, vorteilhaft ist.

2. Staatlicher Handlungsbedarf bei angebotsseitig und nachfrageseitig bestimmter Investitionszurückhaltung

Welche Störimpulse - angebotsseitige oder nachfrageseitige - derzeit in der Bundesrepublik Deutschland die Oberhand gewonnen haben, ist Kern einer Kontroverse zwischen dem DIW und dem Sachverständigenrat ${ }^{1}$. Beide plädieren für mehr Investitionen, haben jedoch unterschiedliche Auffassungen darüber, auf welchem Wege dies erreicht werden kann.

1) Vgl. Sievert, O., Position des Sachverständigenrates, a.a.0., S. $18 \mathrm{ff.;}$ Kirner, W., Position des DIW, in: Vierteljahreshefte zur Wirtschaftsforschung, 1980, s.29 ff. für einen Uberblick vgl. Härtel, H.-H., Die Kontroverse über die "richtige" Vollbeschäftigungsstrategie, in: Wirtschaftsdienst, 1980 , S. $177 \mathrm{ff}$. 
Das DIW verneint, daB gegenwärtig Bedingungen vorliegen, welche den Akzelerationseffekt auBer Kraft setzen; deshalb erwartet es von einer finanzwirtschaftlichen Strategie der Gesamtnachfrageexpansion einen massiven Akzelerationseffekt.

Der Sachverständigenrat hält die Diagnose des DIW für falsch. Er geht davon aus, daB gegenwärtig das Investitionskalkül von störimpulsen dominiert wird, welche durch expansive finanzwirtschaftliche Nachfrageimpulse nicht neutralisiert werden können, da deren Ursprung auf der Angebotsseite $\mathrm{zu}$ orten ist. In diesem Fall erstreckt sich der staatliche Handlungsbedarf nicht auf die Schaffung zusützlichter Güternachfrage, sondern auf die Beseitigung der angebotsseitigen Störimpulse - also auf staatliche Angebotspolitik. Ziel solcher staatlichen Aktivitäten muB eine Verringerung der Ansprüche an das Produktionsergebnis auf ein Niveau sein, welches mehr Produktionsmöglichkeiten rentabel macht ${ }^{1}$ ?

Dies kann dadurch geschehen, daB entweder der staat selbst seine (Abgaben-) Ansprüche mindert (wie beispielsweise bei der Abschaffung der Lohnsummensteuer geschehen) oder dadurch, daB er den Kapitalgebern, Arbeitnehmern und Investoren Hilfestellungen bei der Revision ihrer Ansprüche glbt (zum Beispiel durch eine stärkere staatliche Beteiligung an den Investitionsrisiken).

Staatliche Angebotspolitik setzt - wenn ein Investitionsattentismus angebotsseltig bedingt ist - an den Ursachen der Zurückhaltung der Investoren an und fördert die Investitionstätigkeit. Eine staatliche Nachfragesteuerung ist demgegenüber in diesem Fall wirkungslos; mit einem Akzelerationseffekt ist nicht zu rechnen.

1) Für eine Liste möglicher (nicht nur staatlicher) MaBnahmen vgl. Sievert, O., Die Steuerbarkeit der Konjunktur durch den staat, a.a.0., s. $823 \mathrm{ff}$. 
Viertes Kapitel: Preiseffekte

A. Reales crowding-out

Für den mit einem finanzwirtschaftlichen demand management verfolgten Zweck - eine Erhöhung der Beschäftigung - kommt es nicht auf die nominellen Privatausgaben $(C+I)$ an, sondern auf den Quotienten aus den nominellen Privatausgaben und dem Preisniveau, d.h. auf die realen Ausgaben der Privaten $[(C+I) / P]$ an. Bislang brauchte darauf nicht geachtet $\mathrm{zu}$ werden, da (vom preisinduzierten Vermögenswerteffekt abgesehen) Veränderungen des Preisniveaus ausgeschlossen waren, und es bei konstanten preisen stets auch $z u$ einem realen crowding-out kommt, wenn die nominellen Ausgaben der Privaten sinken. Anders gesehen: Bislang war das Preisniveau konstant, und die reale Güternachfrage der Privaten ist immer deshalb zurückgegangen, weil die nominellen privatausgaben eingeschränkt worden sind.

Die Ursache für ein crowding-out kann jedoch auch in einer Zunahme des Preisniveaus liegen. Wie wichtig gerade dieser Verdrängungseffekt sei, wird immer wieder von den Monetaristen hervorgehoben: "It should surprise no one that the response of prices ... to government spending, neglected in simplified Keynesian analysis, is the mechanism, by which crowding-out occurs"1) Allerdings müssen zwei Bedingungen erfüllt sein, damit es via Preiseffekte zu einem realen crowding-out kommt.

1) Brunner, K., Meltzer, A.H., Government, the Private Sector and Crowding Out, in: The Banker, 1976, S. 767 ; vgl. auch Cansier, D., Einwände gegen die Wirksamkeit der Fiskalpolitik, in: Kredit und Kapital, 1977, S. $58 \mathrm{ff}$. 
Zum ersten müssen die Preise steigen, weil der staat interveniert hat. Bei anderen Verursachern kann eine Verminderung der realen Güternachfrage der Privaten nicht mit dem Signum crowding-out belegt werden. Auf welchen Wegen der staat mit stabilisierungspolitisch motivierten und kreditär finanzierten Nachfrageimpulsen das Preisniveau beeinflussen kann, ist Gegenstand des übernächsten Abschnitts.

Zum anderen dürfen sich die Privaten einem angesichts steigender Preise drohenden realen crowding-out nicht dadurch entziehen, daB sie ihre nominellen Privatausgaben um die Preissteigerungsrate erhöhen. Weshalb man von einer inversen Beziehung zwischen Preisniveau und realen Privatausgaben - auch bei Freiheit von Geldillusion - ausgehen kann, wird im nächsten Abschnitt behandelt. Zudem wird hier ein gesamtwirtschaftliches Preis-Mengen-Diagramm abgeleitet, welches im weiteren Verwendung findet.

B. Zur Abhängigkeit der realen Güternachfrage der Privaten vom Preisniveau

Sind die Privaten frei von Geldillusion, dann hegen sie den Wunsch, im Fall eines Anstiegs des Preisniveaus ihre nominellen Ausgaben um die Preissteigerungsrate aufzustocken. Bei einer konstanten Geldmenge gelingt dies jedoch nur, sofern sich der Geldumlauf beschleunigt. Die dabei auftretende $\mathrm{Zinssteigerungstendenz}$ veranlaBt die Privaten, ihre zinsabhängigen Ausgaben einzuschränken. Im Ergebnis nehmen deshalb die nominellen Privatausgaben nur unterproportional $z u$ und die realen $a b$, wenn das Preisniveau steigt. 
Eine derartige Beziehung zwischen dem Preisniveau und der realen Güternachfrage der Privaten ist implizite auch in unserem Grundmodell enthalten ${ }^{1 !}$ Werden die ISund die LM-Funktion des Grundmodells in realen Größen geschrieben

$$
\begin{aligned}
(2-4-1) Y^{Y}-C^{Y}\left(Y^{Y}-T^{Y}\right)-I^{Y}\left(1^{Y}\right)-\bar{G}^{r} & =0, \\
(2-4-2) \quad L^{Y}\left(Y^{Y}, i^{Y}\right)-\overline{M Z} / P & =0,
\end{aligned}
$$

kann man eine reduzierte Gleichgewichtsbedingung für den Fall eines variablen Preisniveaus in der Form von

$$
(2-4-3) \quad Y^{d r}=Y(P)
$$

ableiten.

Weil eine autonome reale Güternachfrage des Staates unterstellt ist, kommt in der Gleichung (2-4-3) nicht nur zum Ausdruck, wie die insgesamt, sondern auch wie die allein von den Privaten getätigten realen Ausgaben vom Preisniveau abhängen.

Lage und Neigung von Gleichung (2-4-3) werden durch die in realen GröBen formulierten Gleichgewichtsbedingungen von Güter- und Geldmarkt bestimmt. Da sich bei einem variablen Preisniveau dort allein die reale Geldmenge verändert, hat die Nachfragefunktion (2-4-3) in einem Preis-Mengen-Diagramm einen negativen verlauf (vgl. Abb. 6): Sie ist um so flacher (dies bedeutet: die Privaten schränken

1) Die Transformation der IS- und der LM-Funktion in eine preisniveauabhängige Güternachfragefunktion erläutert Brandmiller, S., Zur Integration Zahlungsbilanztheoretischer Ansätze - Eine Makroökonomische Analyse, Mainzer Dissertation, 1979, S. $78 \mathrm{ff}$. 
Abbildung 6: Preis-Mengen-Diagramm mit "monetaristischer" und "keynesianischer" Güterangebotsfunktion

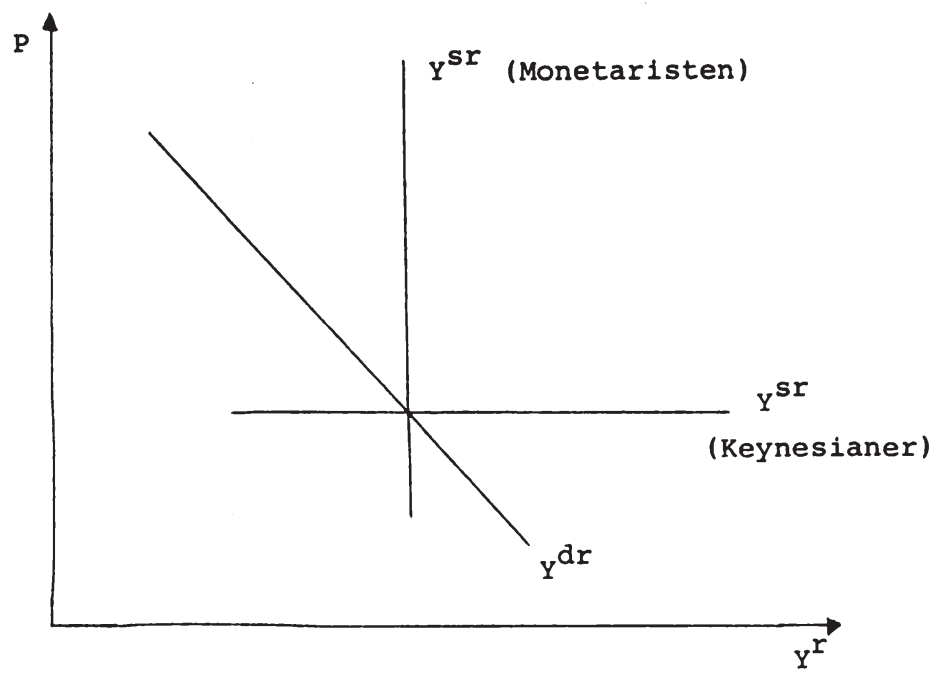

Im Zuge steigender Preise ihre realen Ausgaben um so mehr ein), je (absolut) gröBer die zinselastizität der Investitionen und je (absolut) geringer die zinselastizität der Geldnachfrage ist ${ }^{1}$ ?

1) Wenn, was bisweilen geschieht, bei der Ableitung der preisniveauabhängigen Güternachfragefunktion preisinduzierte Vermögenswerteffekte in die Uberlegungen integriert werden, nimmt die Preisreagibilität der realen Güternachfrage $z u$. Mit steigenden Preisen sinkt zum einen der reale Wert des angesammelten Vermögens, weshalb die Privaten vergleichsweise mehr sparen und weniger konsumieren. Zum anderen wird über die VermögenAllokation-Relation die zinsabhängige Ausgabetätigkeit der Privaten gedämpft. Vgl. hierzu S. 110 oben. 
Wenn exogene Variablen, wie z.B. die reale Güternachfrage des Staates, ihren Wert ändern, kommt es im Preis-MengenDiagramm zu einer Verschiebung der Nachfragefunktion. Um die daraus folgende Preisniveauerhohung zu ermitteln, muß noch eine weitere Funktion in das Preis-Mengen-Diagramm eingefügt werden, nämlich eine preisniveauabhängige Güterangebotsfunktion. An ihrer Steigung macht Milton Friedman in seinem viel diskutierten Aufsatz "A Theoretical Framework for Monetary Analysis" aus dem Jahre 1970 das seiner Ansicht nach konstitutive Unterscheidungsmerkmal zwischen Keynesianern und Monetaristen fest: ${ }^{1)}$ Erstere unterstellen (wie im Grundmodell implizite praktiziert) eine horizontal verlaufende Güterangebotsfunktion. Monetaristen dagegen gehen von einer vertikal verlaufenden Güterangebotsfunktion aus. Um das in realen GröBen formulierte IS-LM-Modell lösen zu können, setzen mithin die Keynesianer das Preisniveau $(\bar{P})$ und die Monetaristen die reale Produktion $\left(\bar{Y}^{r}\right)$ konstant.

1) Vgl. Friedman, M., A Theoretical Framework for Monetary Analysis, a.a.o., s. $31 \mathrm{ff}$.; vgl, auch Burrows, P., The Government Budget Constraint and the Monetarist-Keynesian Debate, a.a.0., S. $67 \mathrm{ff}$. 
C. Preisniveau und kreditär finanzierte staatliche Nachfrageimpulse

1. Finanzwirtschaftlich verursachter Nachfragesog

a) Monetärer Nachfragesog

Für Monetaristen sind Preisniveauvariationen "always and everywhere a monetary phenomenon"1) Grundlage ihrer Uberlegungen ${ }^{21}$ ist die Quantitätsgleichung

$(2-4-4) \quad M \cdot U=Y^{r} \cdot P$

die sie mit der Verwendungsgleichung des Sozialprodukts verknüpfen

$(2-4-5) \quad M \cdot U=Y^{r} \cdot P=C+I+G$

und von.links nach rechts interpretieren: das Preisniveau (P) steigt immer dann, wenn und weil bei konstanter Umlaufsgeschwindigkeit des Geldes (U) die Geldmenge (M) - und damit die von Privaten und staat entfaltete nominelle Güternachfrage $(C+I+G)$ - in einem MaBe zunimmt, mit welche die reale produktion $\left(Y^{r}\right)$ nicht schritt halten kann.

Im Zentrum der monetaristischen Erklärung steht mithin die Geldmenge. Das Preisniveau erhöht sich infolge einer Expansion der Geldmenge, was hier interessiert: die durch eine kreditär finanzierte Vermehrung der Transformationsausgaben verursacht worden ist (finanzwirtschaftlich

1) Friedman, M., The Counter-Revolution in Monetary Theory, First Wincott Memorial Lecture, Institute of Economic Affairs for the Wincott Foundation, Occasional Paper 33, London, 1970, S. 24.

2) Vgl. z.B. Vane, H.R., Thompson, J.L., Monetarism. Theory, Evidence and Policy, a.a.0., s. 72 ff. 
verursachter monetärer Nachfragesog).

Damit ein finanzwirtschaftlich induzierter monetärer Nachfragesog entsteht und dieser sich trotz Unterbeschäftigung nicht in einem Mengen-sondern in einem Preiseffekt niederschlägt, müssen drei Bedingungen erfuilt sein.

Erstens: Es muB die Geldmenge expandieren - und zudem deswegen, weil die finanzwirtschaftliche MaBnahme ergriffen worden ist. Finanzwirtschaftliche Impulse konnten in unserer Wirkungsanalyse bislang nicht via Geldmengeneffekte in den privaten Bereich übertragen werden. Es ist nämlich unterstellt worden, daB die Zentralbank eine Geldmengenpolitik betreibt und deshalb die Geldmenge konstant ist. Von dieser Vorgehensweise wird nun abgewichen. Wir nehmen stattdessen an, daB die Notenbank ein bestimmtes Zinsniveau im Auge hat und sich permissiv im Hinblick auf die Geldmenge verhält. Wenn der Staat zusätzliche Güternachfrage entfaltet, bleibt nun das Zinsniveau konstant. Die Geldmenge kann sich jedoch verändern, und finanzwirtschaftliche Impulse können über Geldmengeneffekte in den privaten Bereich übertragen werden ${ }^{1)}$.

1) "Increased Federal borrowing when added to the credit demands of the private sector, places upward pressure on interest rates. The monetary authority, however, can resist these pressures for a short period of time by buying government securities. Thus, to the extent that "low" interest rates assume a role as an objective of the monetary authorities, deficit financing tends to accelerate the rate of monetary expansion". Carlson, M.K., Large Federal Budget Deficits: Perspective and Prosperity, in: Federal Reserve Bank of St. Louis Review, October 1976, S. 2; vgl, auch Hein, S.E., Deficits and Inflation, a.a.O., S. 5 sowie Willms, M., Volkswirtschaftiche Wirkungen einer zunehmenden Staatsverschuldung, in: Wirtschaftsdienst, 1978, S. 422 und Cassel, D., Wachsende Staatsverschuldung - Wohltat oder Plage?, in: List Forum, 1980, S. 280 f. 
Ebenso wie bel einem crowding-out via zinseffekte fällt der Wähungsbehorrde bei elnem crowding-out via Geldmengeneffekte eine konditionierende Rolle zu: Damit es uber Geldmengeneffekte $z u$ einer Verdrängung privater Ausgaben kommt, muB die Geldmengenexpansion, welche durch das finanzwirtschaftliche Handeln ausgelöst worden ist, von der Notenbank toleriert werden.

Eine Geldmengenausdehnung kann durch direkte und indirekte Giral- oder Zentralbankgeldschöpfungsfinanzierung der staatlichen Güternachfrage besorgt werden.

Die direkte Zentralbankgeldschöpfungsfinanzierung ist in der Bundesrepublik Deutschland der öffentlichen Hand weitgehend verwehrt. Es besteht lediglich die Möglichkeit, sich durch Auflösung zuvor gebildeter Konjunkturausgleichsrücklagen sowie durch Aufnahme von Kassenverstärkungskrediten bis in eine Höhe von ca. $10 \mathrm{Mrd}$. DM direkt bei der Bundesbank Zentralbankgeld zu beschaffen ${ }^{1)}$. Zudem dient der Kassenverstärkungskredit der Uberbrückung von temporären Einnahmelücken innerhalb eines Haushaltsjahres und nicht der Haushaltsfinanzierung.)

Zu einer direkten Giralgeldschöpfungsfinanzierung kommt es dann, wenn sich der Staat bei den Geschäftsbanken gegen Einräumung von Sichtdepositer verschuldet. Da in der Bundesrepublik Deutschland der Bund bei den Geschäftsbanken keine Konten führen darf, hat allerdings diese Art

1) Für den Bund liegt die Höchstgrenze bei 6 Mrd. DM, für die Stadtstaaten Berlin, Bremen und Hamburg bei 80 DM je Kopf der Bevolkerung und fur die sonstigen Länder bei 40 DM je Kopf der Bevölkerung.

2) Einer bislang selten praktizierten Spezies der direkten Zentralbankgeldschöpfungsfinanzierung hat sich in der Bundesrepublik Deutschland der Bundesfinanzminister im Haushaltsjahr 1981 bedient: Die Bundesbank hat $2,3 \mathrm{Mrd}$. DM ihres Gewinns in Höhe von $8,8 \mathrm{Mrd}$. DM an den Bund ausgeschütet. Vgl. hierzu z.B. Friedrichs, H., Bundesbankgewinne machen sinnlich, in: Frankfurter Allgemeine Zeitung vom 29. Juni 1981. 
der Geldschöpfungsfinanzierung hierzulande fur finanzwirtschaftliche StabilisierungsmaBnahmen keine groBe Bedeutung.

Sofern der Staat sich weder bei den Geschäftsbanken gegen Einräumung von Sichteinlagen verschuldet (direkte Giralgeldschöpfungsfinanzierung) noch sich die finanziellen Mittel bei der Zentralbank besorgt (direkte Zentralbankgeldschöpfungsfinanzierung), jedoch die aus dem staatlichen Handeln folgenden Anpassungsreaktionen im monetären Bereich $z u$ einem erhöhten Umlauf an Zentralbankgeld und/oder Giralgeld führen, sind die zusätzlichen staatsausgaben ebenfalls durch Geldschöpfung finanziert worden - jetzt indessen auf indirektem Wege.

Argy ${ }^{1)}$ ist der Frage, ob zwischen dem staatlichen Finanzierungssaldo und der Geldmenge ein Zusammenhang besteht, empirisch nachgegangen. In einem Test, der sich auf sieben Länder und den Zeitraum zwischen 1965 und 1975 erstreckt, wird der staatliche Finanzierungssaldo einmal mit den Notenbankkrediten und einmal mit der Zentralbankgeldmenge (= direkte und indirekte Zentralbankgeldschöpfungsfinanzierung) verknüpft. Als Ergebnis erhält er "... that the view that there is a close link between deficits and monetary growth has little empirical support"2) Zum gleichen Ergebnis kommt Willms: "Uber die Ausdehnung der Staatsschuld ist in der Bundesrepublik keine exzessive Ausdehnung der Geldmenge erfolgt" ${ }^{3}$ ? Willms zieht allerdings von den verschiedenen Formen der Geldschöpfungsfinanzierung nur eine Form, nämlich den Notenbankkredit, ins Kalkül und dieser Form sind, wie dargestellt, in der Bundesrepublik Deutschland enge Grenzen auferlegt.

1) Vgl. Argy, V., Some Notes on Fiscal Policy and Crowding out, a.a.o., s. $62 \mathrm{ff}$.

2) Ebenda, S. 64 .

3) Willms, M., Volkswirtschaftliche Wirkungen einer zunehmenden Staatsverschuldung, a.a.0., s. 443. 
Zweitens: Die Geldmengenexpansion darf nicht durch eine gegenläufige Veränderung der Umlaufsgeschwindigkeit des Geldes kompensiert werden. Wenn dies der Fall ist, steigt zwar die Geldmenge (M), nicht aber der Geldstrom (M.U), und es entsteht kein monetärer Nachfragesog.

Da die Umlaufsgeschwindigkeit des Geldes gleichsam ein Spiegelbild der Kassenhaltungsneigung ist, entscheidet über eine Veränderung der Umlaufsgeschwindigkeit des Geldes infolge der finanzwirtschaftlichen MaBnahme, welchen EinfluB das Handeln des Staates auf die Neigung der Privaten, Kasse zu halten, hat!' Eine zinsinduzierte Variation der Kassenhaltungsneigung ist ausgeschlossen; die Notenbank verfolgt nämlich annahmegemäB eine am zins orientierte Politik. Veränderungen des Vermögens der Privaten und ihrer Erwartungen können den Wunsch auslösen, die Kassenhaltung aufzustocken. Wenn sich das Vermögen erhöht, wollen, wie dargelegt ${ }^{2}$, die Privaten nämlich den Vermögenszuwachs zum Teil auch in liquider Form halten. Hinzu kommt, worauf schon Keynes hinwies und was noch erörtert wird ${ }^{3)}$, daB möglicherweise das Mibtrauen, welches die Privaten gegenüber einem zunehmenden Staatsschuldenstand hegen, auf die Liquiditätspräferenz durchschlägt und sie deswegen versuchen, ihr Portefeuille in Richtung ertragslosem, aber kurssicherem Geld umzustrukturieren.

Drittens: Die Geldmengenexpansion muB sich trotz Unterbeschäftigung in einem Preiseffekt und darf sich nicht in einem Mengeneffekt niederschlagen. In welchem AusmaB durch einen monetären Nachfragesog das Preisniveau steigt, hängt von der Neigung der Güterangebotsfunktion in den Bereichen $a b$, in welchen die Geldmengenvermehrung zu zusätzlichen Ausgaben führt. Preiserhöhungen auf breiter Front sind

1) Vgl. Hein, S.E., Deficits and Inflation, a.a.O., S. $4 \mathrm{ff}$.

2) Vgl. S. $61 \mathrm{ff}$. oben

3) Vgl. S. 158 ff. unten 
dann zu erwarten, wenn die Ursachen der Unterbeschäftigung angebotsseitig bedingt sind. In diesem Fall ist die gesamtwirtschaftliche Güterangebotsfunktion in einem Preis-Mengen-Diagramm auch bei Unterbeschäftigung starr, und ein monetärer Nachfragesog verpufft in einem Preisniveauschub.

Ein steigendes Preisniveau kann allerdings angebotsseitigen Störimpulsen an Schärfe nehmen und zwar insoweit wie diejenigen, welche Anspruch auf das Produktionsergebnis erheben, übersehen, daB sich mit zunehmenden Preisen auch das bewertete Produktionsergebnis erhöht. Bleibt ihr absoluter Anspruch an das Produktionsergebnis deswegen konstant, verschieben sich die relativen Ansprüche $z u$ ihren Ungunsten, anders gewendet: ihre preisbereinigten absoluten Ansprüche nehmen ab.

Passen die an der Produktion Beteiligten ihre nominellen Ansprüche mit einer geringeren Rate an als mit derjenigen, um die das Preisniveau steigt, hat im PreisMengen-Diagramm die Güterangebotsfunktion keinen senkrechten, sondern einen nach rechts geneigten Verlauf, und es entsteht folglich - zumindest temporär - ein trade off zwischen Preisniveau und Beschäftigung. Die realen Ausgaben der Privaten werden nur partiell und nicht total verdrängt.

Mit einer Erhöhung des Preisniveaus ist bei Unterbeschäftigung auch dann $z u$ rechnen, wenn die Gründe für die Unterbeschäftigung auf der Güternachfrageseite liegen, jedoch auf Teilmärkten die Produktionsfaktoren vollbeschäftigt.sind und dort die zusätzliche Güternachfrage 
entfaltet wird". Derartige partielle Preisschube sind eine häufige Begleiterscheinung eines monetăren Nachfragesogs, welcher durch geldpolitische MaBnahmen verursacht 1st. Wenn der monetare Nachfragesog jedoch Folge staatlichen Handelns ist, mübten sich eigentlich solche Preiserhöhungen vermeiden lassen. Denn der staat hat es prinzipiell auf der ersten Stufe des Wirkungsprozesses in der Hand, auf welche partiellen Güterangebotsfunktionen die zusätzlichen Ausgaben treffen. Faktisch besteht indessen dieser Vortei]. nicht. Die staatiichen Ausgabenprogramme konzentrieren sich regelmäBig auf wenige Teilmärkte (in der Bundesrepublik Deutschland hauptsächlich auf den Baumarkt). In der Praxis ist die öffentliche Hand mithin kaum in der Lage, eine Vermehrung der Güternachfrage so auf die einzelnen Teilmärkte aufzuteilen, daB partielle preisschübe vermieden werden.

\section{b) Güterwirtschaftlicher Nachfragesog}

Keynesianer interpretieren Gleichung (2-4-5) - umgekehrt $\mathrm{zu}$ den Monetaristen - von rechts nach links: Das Preisniveau (P) steigt, wenn und weil die nominelle Güternachfrage $(C+I+G)$ - und damit der Geldstrom $(M \cdot U)$ - zunimmt und auf eine nicht ausdehnungsfähige reale produktion $\left(Y^{r}\right)$ trifft. In dieser wie auch in der monetaristischen Sichtweise sind Preisniveauerhöhungen jeweils Folge eines Nachfragesogs: Keynesianer erblicken jedoch die Ursache dafür nicht in einer Zunahme der Geldmenge, sondern machen für den Nachfragesog die Güternachfrager verantwortlich. Nicht auf die Geldmenge, sondern auf das Ausgabeverhalten

1) Vgl. Koch, W.A.S., Lang, E., Crowding-out und staatliches debt management, a.a.0., S. 53 f., Schmidt, K., రffentliche Neuverschuldung in der Rezession - neue Erfahrungen und alte Einsichten, in: రffentliche Verschuldung und Kapitalmarkt, Schriftenreihe des Instituts für Kapitalmarktforschung an der J.W. Goethe-Universität, Frankfurt, 1976, S. $47 \mathrm{ff}$. 
der Privaten und - was hier interessiert - des Staates kommt es an ${ }^{1)}$ (finanzwirtschaftlich verursachter güterwirtschaftlicher Nachfragesog).

Damit ein finanzwirtschaftlich verursachter güterwirtschaftlicher Nachfragesog entsteht und deshalb das Preisniveau steigt, müssen zwei Bedingungen erfültt sein.

Erstens: Es muB sich die gesamtwirtschaftliche nominelle Güternachfrage erhöhen. Dies setzt im monetären Bereich eine entsprechende Zunahme des Geldstromes voraus. Bei einer konstanten Umlaufsgeschwindigkeit des Geldes muB die Notenbank die Guternachfrageste1gerung durch eine Geldmengenexpansion monetär alimentieren.

Zweitens: Der güterwirtschaftliche Nachfragesog muB zu einem Preiseffekt und darf nicht zu einem Mengeneffekt führen. DaB es durch zusätzliche Transformationsausgaben, welche nicht allokationspolitisch, sondern stabilisierungspolitisch motiviert sind, zu einer Rivalität zwischen der privaten und der staatlichen Guternachfrage kommt, beruht letztlich auf einem Diagnosefehler auf Selten des Staates: Er unterliegt der Illusion, er könne mit einer Güternachfrageausdehnung Mengeneffekte erzeugen, weil seine Ausgaben auf eine ausdehnungsfähige reale Produktion träfen. Tatsächlich 1st dies jedoch nicht der Fall, sei es, weil die Unterbeschäftigung an-

1) Der Sachverhalt ist der gleiche wie bel dem monetären Nachfragesog, nur die Interpretation ist eine andere: Monetaristen sehen in der Geldmengenveränderung eine conditio per quam, Keynesianer - bei konstanter Umlaufsgeschwindigkeit des Geldes - nur eine conditio sine qua non. 
gebotsseitig bedingt ist ${ }^{1}$, sei es, weil bei einer nachfrageseitig verursachten: generellen Unterbeschäftigung auf Teilmärkten die Produktionsfaktoren vollbeschäftigt sind.

2. Finanzwirtschaftlich verursachter Angebotsdruck

Anhänger der Angebotsdrucktheorie erklären eine Preisniveauerhöhung von der Güterangebotsseite her. Das Preisniveau steigt, wenn und weil die Preise für alternative Mengen von den Anbietern nach oben gesetzt werden. Einen Angebotsdruck kann der staat mittelbar und unmittelbar erzeugen. Die unmittelbare EinfluBnahme, d.h. staatliches Guterangebot gegen Entgelt ist hier nicht zu behandeln. Durch eine stabilisierungspolitisch motivierte Ausdehnung der Transformationsausgaben ergeben sich allein mittelbare Einflußmöglichkeiten, nämlich dergestalt, daB infolge der staatlichen Aktivität die privaten Güteranbieter animiert werden, ihre Angebotspreise $\mathrm{zu}$ erhöhen (finanzwirtschaftlich verursachter Angebotsdruck).

Zum ersten entsteht dadurch ein Anǵebotsdruck, daB das staatliche Handeln regelmäBig eine zinssteigerungstendenz auslöst. Im Zuge der Verschiebung der Knappheitsverhältnisse auf den monetären Märkten nehmen also die Ansprüche der

1) Art. 109 GG. Abs. 2, nach dem Bund und Länder "bei ihrer Haushaltswirtschaft den Erfordernissen des gesamtwirtschaftlichen Gleichgewichts Rechnung zu tragen" haben, kann im Lichte solcher Uberlegungen nicht mehr - wie meistens geschehen - ausschlieBlich als Aufforderung $z u$ einem finanzwirtschaftlichen demand management verstanden werden, "weil defizitfinanzierte ... Ausgabenprogramme angebotsseitig bedingte Beschäftigungsprobleme nicht beseitigen können". Brenner, G., Haury, C.E., Lipp, E.M., Staatsverschuldung und Verfassung. Bestandsaufnahme und Neubesinnung, in: Finanzarchiv, 1980, S. 250. 
Fremd- und Eigenkapitalgeber an das Produktionsergebnis zu. Höhere Finanzierungskosten veranlassen die Güteranbieter dazu, ihre Preisforderungen nach oben zu revidieren.

Zum zweiten kann man die Ansprüche der Unternehmer an das Produktionsergebnis kausal mit der finanzwirtschaftlichen Maßnahme verknlipfen. Wenn diese ad hoc ergriffen wird, wähnen sich möglicherweise die Unternehmer einem erhöhten Risiko dartiber ausgesetzt, in welchem AusmaB in der Zukunft mit ăhnlichen staatlichen Aktivitäten zu rechnen ist ${ }^{1)}$. Stocken sie als Ausgleich dafur thre Risikoprämie auf, ist durch die finanzwirtschaftliche MaBnahme ein Angebotsdruck erzeugt worden.

Zum dritten lassen sich auch mit stabilem Geldwert nicht vereinbare Ansprüche der Arbeitnehmer an das Produktionsergebnis auf die finanzwirtschaftliche Maßnahme zurückführen: Wird im zielkatalog der Finanzpolitik der Vollbeschäftigung erste Priorităt eingeräumt ${ }^{2)}$, können die Arbeitnehmer das Risiko eines zu hohen Lohnabschlusses - eine Einschränkung der Beschäftigung - ignorieren. Denn aufgrund der Vollbeschäftigungsgarantie entfällt die Möglichkeit, daß der ausgehandelte Lohnzuwachs via Angebotsdruck einen Preisschub auslöst und bei konstantem Geldstrom einen negativen Mengeneffekt zeitigt. Die Arbeitnehmer können nämlich darauf vertrauen, daB bei einer Tendenz zur Unterbeschäftigung vom staat expansive Maßnahmen ergriffen werden, um die abgegebene Be-

1) Vgl. Sachverständigenrat zur Begutachtung der gesamtwirtschaftlichen Entwicklung, Jahresgutachten 1975, Bundestagsdrucksache 7/4326, Ziff. 382 und Ziff. 394.

2) In der Bundesrepublik Deutschland war dies in der letzten Zelt hauptsächlich in den ersten Jahren nach dem politischen Machtwechsel im Jahr 1969 der Fall. Vgl. hierzu Kloten, N., Barth, H.J., Ketterer, K.H., Vollmer, R., Zur Entwicklung des Geldwertes in Deutschland, Tübingen, 1980, S. 53 f. und S. $143 \mathrm{f}$. 
schäftigungsgarantie zu erfüllen"! Im Fall zusätzlicher Transformationsausgaben - was hier interessiert - haben diese dann quasi ex ante ein crowding-out bewirkt. Anders gewendet: Die reale Güternachfrage der Privaten ist gesunken, bevor und weil zusätzliche staatliche Güternachfrage entfaltet worden ist.

Anhand des Preis-Mengen-Diagramms läBt sich diese These folgendermaBen darstellen (vgl. Abb. 7). In der Ausgangslage hat sich im Zusammenspiel von Guterangebot $\left(Y_{0}^{S r}\right.$ ) und Guternachfrage $\left(Y_{0}^{d r}\right)$ eine reale Produktion in Horhe von $\left(Y_{0}^{r}\right)$ eingestellt. Tätigt der staat zusätzliche Transformationsausgaben, verlagert sich die Nachfragefunktion z.B. auf $\mathrm{Y}_{1}^{\mathrm{dr}}$. Weil sie mit dieser Transformationsausgabenvermehrung gerechnet haben, konnten die lohnpolitischen Akteure schon vorher Lohnzuwächse vereinbaren, welche die Angebotsfunktion bis auf $\mathrm{Y}_{1}^{\mathrm{sr}}$ nach oben verschoben haben. Trotz des damit erzeugten Angebotsdrucks bleiben negative Mengeneffekte aus.

Dennoch sind die realen Ausgaben der Privaten gesunken. Sie haben um den Betrag der realen Güternachfrage, welche vom Staat zusätzlich entfaltet worden ist, abgenommen. Ohne das Wissen um die Beschäftigungsgarantie,

1) "Die Zuweisung beschäftigungspolitischer Verantwortung an die Finanzpolitik wirkt zurück auf die Neigung des Marktsystems zu Beschäftigungsproblemen, well in der Lohnpolitik gröBere Beschäftigungsrisiken eingegangen werden können, wenn der staat sie abzufangen hat". Sievert, O., LäBt sich konjunkturgerechtes Verhalten der öffentlichen Gebietskörperschaften messen?, in: Quartalshefte der Girozentrale, 2-3/1978, S. 59; vgl. auch Phelps-Brown, H., A Non-Monetarist View of the Pay Explosion, in: The Three Banks Review, 1975, S. 3 ff.; Merklein, R., "Wenn die Lebensgeister trübe werden", in: Der Spiegel, 1978, Nr. 37, S. $109 \mathrm{ff}$. 
Abbildung 7: Staatliche Beschäftigungsgarantie und Lohnansprüche

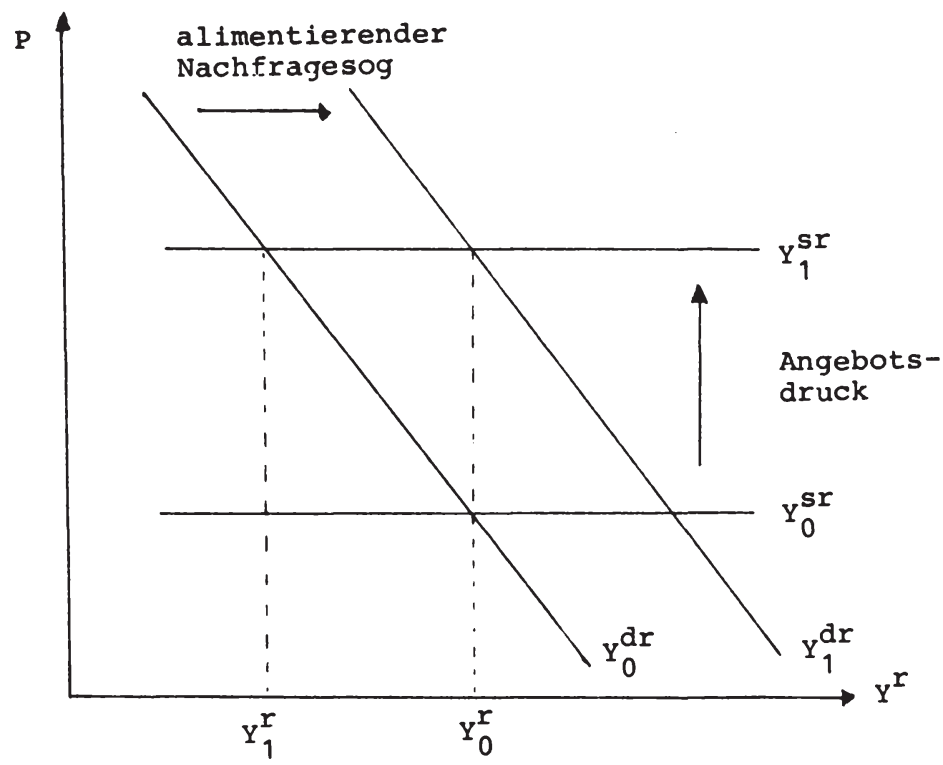

also die Erwartung einer Ausdehnung der Güternachfrage, hätten die Arbeitnehmer, wenn ihnen daran gelegen gewesen wäre, negative Beschäftigungseffekte $\mathrm{zu}$ vermeiden, nur Lohnzuwächse ausmachen können, welche die Angebotskurve in ihrer Ausgangslage $\mathrm{Y}_{0}^{\mathrm{Sr}}$ belassen hätte.

Allerdings erfüllen zusätzliche Transformationsausgaben nur dann ihren Zweck, wenn durch sie tätsächlich ein Nachfragesog entsteht, d.h. sich die Nachfragefunktion 
nach rechts verschiebt. Mithin muB der Geldstrom zunehmen; die währungsbehörde muB also die von den Arbeitnehmern und-gebern diktierte Preisniveauerhöhung monetär alimentieren ${ }^{1}$ ?

1) Gordon nennt die Währungsbehörde deshalb "Anbieter von Inflation" und Unternehmer und Arbeitnehmer "Nachfrager von Inflation". Das tatsächliche Angebot an Inflation hängt davon $a b$, inwieweit die geldpolitischen Entscheidungsträger sich um des Beschäftigungszieles willen von dem geplanten (Null-) Angebot an Inflation abbringen lassen. Vgl. Gordon, R.J., The Demand for and the Supply of Inflation, in: Journal of Law and Economics, 1975 , S. $807 \mathrm{ff}$. 
Fünftes Kapitel: Erwartungseffekte

A. Erwartungen als Bestimmungsgrund der privaten Ausgaben

Die Ausgaben der Privaten werden nicht zuletzt von ihren Erwartungen bestimmt. Häufig nimmt man in Wirkungsanalysen an, die Erwartungen hätten einen festen wert und seien damit von finanzwirtschaftlichen Nachfrageimpulsen unabhängig.

Dieses auch im Grundmodell praktizierte Vorgehen hat dort zur Konsequenz: Zwar spielen Erwartungen für die Dispositionen der Privaten eine wichtige Rolle, von finanzwirtschaftlichen MaBnahmen werden sie jedoch nicht beeinfluBt. So vergleichen zum Beispiel die privaten Investoren bei ihren Entscheidungen die ErwartungsgröBe Grenzleistungsfähigkeit des Kapitals mit dem aktuellen Marktzins und tätigen Ausgaben, sofern die Grenzleistungsfähigkeit des Kapitals den Marktzins übersteigt. Wenn der Staat seine kreditfinanzierte Güternachfrage ausdehnt, nimmt er allein über eine Erhöhung des gegenwärtigen Marktzinses EinfluB auf den Umfang der von den Privaten entfalteten Investitionsgüternachfrage - nicht via einer Veränderung der (konstant gesetzten) ErwartungsgröBe Grenzleistungsfähigkeit des Kapitals. Diese restriktive Annahme einer Unabhängigkeit der Erwartungen der Privaten von finanzwirtschaftlichen MaBnahmen wird im folgenden aufgehoben.

Es werden drei Thesen erörtert. Bei den ersten beiden steht das Zukunftsvertrauen der Privaten im Mittelpunkt; dieses kann unter finanzwirtschaftlichen Nachfrageimpulsen leiden, und die beabsichtigte Beschäftigungswirkung wird abgeschwächt. Bei der dritten geht es um ein Tempoproblem. Gemäß der These der Rationalisten gibt es, wenn die Erwartungen rational gebildet werden, nicht einmal kurzfristig einen trade-off 
zwischen Beschäftigung und Preisniveau, welcher von der Finanzpolitik genutzt werden könnte.

Liegen die Ursachen eines crowding-out in Erwartungseffekten, vollzieht sich die Verdrängung privater Ausgaben stets in zwei Schritten: Der zweite ist eine erwartungsinduzierte Umdisposition; die Privaten reagieren auf eine Veränderung ihrer Erwartungen so, daB es zu einer Abnahme ihrer Ausgaben kommt. Zuvor ändern sie im ersten Schritt wegen der vermehrten staatlichen Güternachfrage ihre Erwartungen. Wie diese Veränderung ausfällt, hängt auch davon $a b$, auf welche Art und Weise sich bei den Privaten Erwartungen bilden.

B. Zum Bilden von Erwartungen

In der Literatur herrschen unterschiedliche Einschätzungen darüber vor, was eine Rolle spielt, wenn sich bei den Privaten Erwartungen bilden. Meistens wird zwischen rationalen und autoregressiven Erwartungen unterschieden. Zudem sind psychologische Faktoren wichtig.

Nach Meinung der Rationalisten ${ }^{1)}$ gründen die privaten Wirtschaftssubjekte ihre gedankliche Vorstellung von der künftigen ökonomischen Entwicklung auf ihre Kenntnisse über die Struktur des Wirtschaftsprozesses. Die Privaten haben beim Bilden von Erwartungen gleichsam ein ökonometrisches

1) Für einen Uberblick vgl. Shiller, R.J., Rational Expectations and the Dynamic Structure of Macroeconomic Models, in: Journal of Monetary Economics,1978, S. 1 ff.; Kühn, B., Rationale Erwartungen und Stabilisierungspolitik, BadenBaden, 1979; Neumann, M.J.M.., Rationale Erwartungen in Makromodellen, in: Zeitschrift für Wirtschafts- und Sozialwissenschaften, 1979, S. 371 ff.; Klausinger, H., Rationale Erwartungen und die Theorie der Stabilisierungspolitik, Bern, Frankfurt am Main, Las Vegas, 1980, S. 49 ff.; McCallum, B.T., Rational Expectations and Macroeconomic Stabilization Policy, in: Journal of Money, Credit, and Banking, 1980 , S. $716 \mathrm{ff}$. 
Modell vor Augen. Rational geformte Erwartungen entsprechen folglich den sich aus der Modellanalyse ergebenden Erwartungswerten ${ }^{1 !}$.

Rationale Erwartungen ( und Erwartungswerte) stimmen indes nicht unbedingt mit den sich tatsächlich ergebenden Werten überein. Denn auf die sich tatsächlich ergebenden Werte nehmen auch zufällige Ereignisse Einfluß. In Modellen mit rationalen Erwartungen wird immer unterstellt, die zufälligen Ereignisse seien normalverteilt. Ihre Erwartungswerte sind dann Null, und rationale Erwartungen werden von zufälligen Ereignissen nicht beeinfluBt. Aus Sicht der Privaten hat auch eine Finanzpolitik, die nicht einem bestimmten Handlungsmuster folgt und aus diesem Grund für die Privaten nicht antizipierbar ist, Zufallscharakter. Deshalb weichen bei solchen finanzwirtschaftlichen MaBnahmen die rationalen Erwartungen der Privaten von den sich tatsächlich ergebenden werten $a b$.

Trifft dagegen die Hypothese einer autoregressiven Erwartungsbildung ${ }^{2)} z u$, sind die Wirtschaftssubjekte nicht prospektiv orientiert, sondern retrospektiv und lassen sich beim Formen von Erwartungen von ihren Erfahrungen in der Vergangenheit leiten. Ublicherweise wird davon ausgegangen, die vergangenen Ereignisse hätten dabei ein um so gröBeres Gewicht, je dichter sie an der Gegenwart liegen.

1) Rationale Erwartungen sind von Muth in die Literatur eingeführt worden: "... expectations, since they are informed predictions of future events, are essentially the same as the predictions of the relevant economic theory". Muth, J., Rational Expectations and the Theory of Price Movements, in: Econometrica, 1961 , S. 316.

2) Für einen Uberblick vgl. z.B. Kühn, B., Rationale Erwartungen und Wirtschaftspolitik, a.a.0., s. 27 ff. Ein prominenter Spezialfall autoregressiver Erwartungen sind adaptive Erwartungen. Hiernach korrigieren die Wirtschaftssubjekte ihre Zukunftseinschätzund um einen konstanten Anteil der jeweils festgestellten Abweichung des erwarteten Wertes vom tatsächlich eingetretenen wert. 
Psychologischen Faktoren wird sowohl bei rationalen als auch bei autoregressiven Erwartungen keine Bedeutung beigemessen. Insbesondere, weil es um eine Analyse der Wirkungen einer staatlichen Maßnahme geht, durch die der Staatsschuldenstand steigt, muB allerdings vermutet werden, daB bei autoregressiven und rationalen Erwartungen Gesichtspunkte ignoriert werden, welche für die Erwartungen, die sich bei den Privaten infolge staatlicher Aktivitäten bilden, wichtig sind. Gerade in der Bundesrepublik Deutschland ist nämlich der öffentliche Kredit als eine "Domäne psychologischer Betrachtung" 1 ) anzusehen. "There is a great deal of emotion in people's attitudes toward the public debt"2). Psychologische Faktoren dürfen bei einer wirkungsanalyse kreditär finanzierter staatlicher Aktivitäten nicht aus dem Blick gelassen werden.

C. Crowding-out durch Erwartungseffekte

1. Finanzwirtschaftliches demand management und Zukunftsvertrauen

a) Die These von Keynes

Wie in den dogmenhistorischen Ausführungen schon angedeutet, war sich Keynes durchaus der Möglichkeit bewuBt, daB die stabilisierungspolitische Wirksamkeit eines finanzwirtschaftlichen demand management durch konterkarierende Erwartungseffekte abgeschwächt werden kann: "... the Government programme may, through its effect on 'confidence' increase liquidity-preference or diminish the marginal

1) Schmölders, G., Finanzpsychologie, in: Finanzarchiv, 1951 , S. 31.

2) Ott, D.J., Ott, A.F., Fiscal Policy and National Debt, in: Smith, W.L., Teigen, R.L. (Hrsg.), Readings in Money, National Income, and stabilization Policy, Homewood, Ill., $1974^{3}$, S. 338 . 
efficiency of capital, which, again, may retard other investment unless measures are taken to offset it" ${ }^{1}$ ?

Worauf es Keynes ankam, war, daB die Anbieter und Nachfrager von Finanzkapital Zukunftsvertrauen verlieren können, wenn der staat stabilisierungspolitisch tätig wird ${ }^{2}$ ! Demnach nehmen negative Erwartungseffekte ihren Weg über den Finanzkapitalmarkt.

Glauben die Privaten, daB staatliches Handeln die wirtschaftliche Entwicklung nicht verbessert, sondern verschlechtert, und sinkt aus diesem Grund infolge staatlicher Maßnahmen ihr Zukunftsvertrauen, wird einmal, so Keynes, die Neigung der Geldnachfrager zunehmen, lieber (zinslose) Kasse zu halten, anstatt ihr Geld gegen (zinstragende) Forderungen auf unsicherer gewordene zukünftige Rückflüsse an Investoren $z u$ verleihen. Sie werden folglich Kreditkontrakte nur noch $z u$ vergleichsweise höheren $\mathrm{zinsen}$ eingehen. Zum anderen wird es für Investoren riskanter, jetzt Ausgaben in der Hoffnung auf zukünftige Erträge zu tätigen. Die Grenzleistungsfähigkeit des Kapitals nimmt mithin ab. Beides führt dazu, daß erst für die mittlere Frist erwartete negative Konsequenzen des staatlichen Handelns schon in der Entstehungsphase des Defizits die private Investitionstätigkeit dämpfen?'

1) Keynes, J.M., The General Theory of Employment, Interest and Money, a.a.0., S. 120.

2) Vgl. hierzu auch Sievert, O., Die Steuerbarkeit der Konjunktur durch den Staat, a.a.o., S. $810 \mathrm{ff}$; Carlson, K. M., Spencer, R.W., Crowding Out and its Critics, a.a.O., S. 5 f.; Vane, H.R., Thompson, J.L., Monetarism. Theory, Evidence and Policy, a.a.0., S. 137.

3) "And, if there is disbelief in the benefits of a deficit, then the new money spent by the government may well be more offset by additional withdrawls of private money which would otherwise be spent". Smith, D.T., Is Deficit Spending Practical?, in: Harvard Business Review, 1939, S. 38 . 
Die von Keynes befürchteten kontraproduktiven Erwartungseffekte können in das Grundmodell integriert werden. Dazu wird das staatliche Defizit als Verhaltensargument in der Geld- und in der Investitionsgüternachfragefunktion berücksichtigt ${ }^{1}$ ?

$(2-5-1) \quad I=I\left(i, B-\bar{B}_{-1}\right) \quad$ mit $I_{B}<0$,

$(2-5-2) \quad L=L\left(Y, i, B-\bar{B}_{-1}\right)$ mit $L_{B}>0$

Als modifiziertes Grundmodell ergibt sich dann:

$$
\begin{aligned}
& (2-5-3) \quad Y-C(Y-T)-I\left(i, B-\bar{B}_{-1}\right)-\bar{G}=0 \text {, } \\
& (2-5-4) \quad \mathrm{L}\left(\mathrm{Y}, \mathrm{i}, \mathrm{B}-\overline{\mathrm{B}}_{-1}\right)-\overline{\mathrm{MZ}}=0 \text {, } \\
& (1-4-24) \bar{G}-T(Y)-\left(B-\bar{B}_{-1}\right)=0 \text {. }
\end{aligned}
$$

Man erhält nun als Privatausgabenmultiplikator einer kreditär finanzierten Erhöhung der Transformationsausgaben:

$$
(2-5-5) \frac{d(C+I)}{d \bar{G}}=\frac{1-\frac{I_{i}}{L_{i}} L_{B}+I_{B}}{\left[1-C_{Y} v\left(1-T_{Y}\right)\right]-\left(\frac{I_{i} L_{B}}{L_{i}}-I_{B}\right) T_{Y}+\frac{L_{Y} I_{i}}{L_{i}}}-1 .
$$

wird unterstellt, $\operatorname{daB} \mathrm{T}_{\mathrm{Y}}=0$ ist, unterscheidet sich dieser Privatausgabenmultiplikator von dem des Grundmodells (1-4-33) nur durch zwei zusätzliche Terme im zăhler. In ihnen kommen die beiden von Keynes erwähnten Erwartungseffekte zum Ausdruck: Eine Erhöhung des Staatsschuldenstandes kann unmittelbar über eine erwartungsinduzierte Abnahme der Investitionsgüternachfrage $\left(I_{B}<0\right)$ und mittel-

1) Vg?. für eine ähnliche Vorgehensweise Cebula, R.J., Deficit Spending, Expectations, and Fiscal Policy Effectiveness, in: Public Finance, 1973, s. $362 \mathrm{ff}$. 
bar uber eine erwartungsinduzierte Zunahme der Geldnachfrage $\left(I_{i} / L_{i} \cdot L_{B}>0\right)$ die private Ausgabentätigkeit dämpfen. Im IS-LM-Schema wirken sich diese Erwartungseffekte in einer Linksverschiebung beider Gleichgewichtskurven und damit in einer Verdrängung der privaten Ausgaben aus. Bei hinreichenden Werten, d.h., wenn die Summe der (absoluten) Werte der beiden zusätzlichen Terme im zähler gröBer Eins ist, wird gar ein übermäBiges crowdingout erzeugt.

Die Frage, welche Wirkungen die Privaten gedanklich mit finanzwirtschaftlichen Nachfrageimpulsen verknüpfen, damit es zu einem Verlust an Vertrauen in die zukunft kommt, beantwortet Keynes mit einem schlichten Verweis auf eine "confuse psychology"1! Er rückt mithin psychologische Faktoren in den Vordergrund 2 ! Welche Rolle diese praktisch spielen können, wenn die Privaten die Folgen einer auf dem Kapitalmarkt finanzierten staatlichen Aktivität prognostizieren, macht beispielsweise die gegenwärtig in der Bundesrepublik Deutschland $\mathrm{zu}$ beobachtende Furcht vor einer möglichen Währungsreform oder einem Staatsbankrott deutlich. Bei einer sachlichen Betrachtung besteht dafür wenig AnlaB ${ }^{3}$ ?

1) Keynes, J.M., The General Theory of Employment, Interest and Money, a.a.0., s. 120 .

2) Für politisch-psychologische Grenzen der öffentlichen Kreditnahme vgl. Ziffzer, S., Okonomische, finanzwirtschaftliche, politisch-psychologische und gesetzliche Grenzen der Kreditaufnahme des Bundes, der Länder und der Gemeinden, Forschungsinstitut für Wirtschaftspolitik an der Universität Mainz, unveröffentlicht, 1979, S. 177 ff. sowie derselbe, Grenzen der Staatsverschuldung, in: Zeitschrift für das gesamte Kreditwesen, 1980, S. 500 ff.

3) "Was immer die Visionen der Theoretiker und Politiker beim Deficit Spending sein mögen, die Visionen der öffentlichen Meinung sind fundamental anderer, und zwar negativster Art". Richebächer, K., Das süBe Gift des Deficit Spending, Währungen und Kreditmärkte, Nr. 64, Frankfurt, 1975, S. 12 . Vgl. dazu auch Surminski, A., Was die Deutschen fürchten, in: Frankfurter Allgemeine Zeitung vom 31.5.1978 und Brestel, H., Die Angst vor dem 'Gelduntergang', in: Frankfurter Allgemeine Zeitung vom 6.6.1981. 
b) Die These des Sachverständigenrates

Vom Sachverständigenrat wird die Effizienz eines finanzwirtschaftlichen demand management aus zwei Gründen in Zweifel gezogen. Der eine von beiden ist schon dargelegt worden ${ }^{1)}$ : Liegen die Wurzeln für eine Unterbeschäftigung in angebotsseitigen Störimpulsen, verpuffen Nachfrageimpulse in einem Preisniveauschub, ohne daB mehr produziert und mehr investiert wird. Weil die Unternehmer trotz Unterbeschäftigung - die Produktion nicht ausdehnen, werden die realen Ausgaben der Privaten via Preiseffekte um den Betrag der realen Zusatzausgaben des Staates verdrängt, und es findet ein vollständiges, reales crowdingout statt. Der andere Grund sind negative Erwartungseffekte; hierum geht es im folgenden.

Die These des Sachverständigenrates ${ }^{2)}$, durch kontraproduktive Erwartungseffekte könnten einem finanzwirtschaftlichen demand management Wirkungsgrenzen gezogen werden, beruht zum einen auf der Hypothese, die privaten Konsumenten und Investoren würden sich bei ihren aktuellen Ausgabeentscheidungen an mittelfristig erwarteten Haushaltseinkommen und Gewinnen orientieren und zum anderen auf der Annahme, die Privaten würden die erwarteten Folgen

1) Vgl. S. 139 f. oben sowie Sachverständigenrat zur Begutachtung der gesamtwirtschaftlichen Entwicklung, Jahresgutachten 1976/77, a.a.0., Ziff. 272 ff.; derselbe, Mehr Wachstum, mehr Beschäftigung, Jahresgutachten 1977/78, a.a.O., Ziff. $236 \mathrm{ff.;}$ derselbe, Jahresgutachten 1978/79, a.a.0., Ziff. $266 \mathrm{ff}$. sowie Sievert, O., Die Steuerbarkeit der Konjunktur durch den staat, a.a.o., s. $818 \mathrm{ff.;}$ Brenner, G., Haury, C.E., Lipp, E.M., Staatsverschuldung und Verfassung, a.a.0., s. $248 \mathrm{ff}$.

2) Vgl. hauptsächlich Sachverständigenrat zur Begutachtung der gesamtwirtschaftlichen Entwicklung, Jahresgutachten 1975, a.a.O., Ziff. 228, $380 \mathrm{ff}$. und 423; derselbe, Jahresgutachten 1978/79, a.a.0., Ziff. 308 und 310 ; vgl. auch Sievert, 0., Die Steuerbarkeit der Konjunktur durch den Staat, a.a.0., S. $810 \mathrm{ff.}$ sowie Nölling, w., Staatsverschuldung ohne Ende?, in: Kredit und Kapital, 1979, S. $478 \mathrm{ff}$. 
eines finanzwirtschaftlichen demand management anhand von Erfahrungen in der Vergangenheit abschätzen. Waren diese unerfreulich, attestieren die privaten Wirtschaftssubjekte also dem staat eine verschlechternde EinfluBnahme auf die zukünftige wirtschaftliche Entwicklung, verlieren sie Zukunftsvertrauen, und es nehmen ihre mittelfristig erwarteten Gewinne und Haushaltseinkommen - und deshalb die aktuellen Ausgaben - $a b$, wenn der staat Nachfrageimpulse entfaltet.

Dann ist der Staat - wie nach Einschätzung des Sachverständigenrates z.B. gegenwärtig in der Bundesrepublik Deutschland ${ }^{1)}$ - in eine Lage geraten, in welcher er "fürchten muB, eine expansive Finanzpolitik werde sich über eine ... Verschlechterung der Erwartungen sogar gegen sich selber kehren, also im Endeffekt kontraproduktiv wirken"?)

Weshalb die Privaten Zukunftsvertrauen verlieren, wenn der staat versucht, die gesamtwirtschaftliche Güternachfrage anzuheben, begründet der Sachverständigenrat damit, in der letzten Dekade sei hierzulande bei den privaten Konsumenten und Investoren die gedankliche Vorstellung entstanden, daB "die kurzfristige Ausweitung der Ansprüche des staates auch für die mittlere Frist" ${ }^{3)}$ anhalte und daB "Staatsdefizite ... nur die Inflation ...

1) Vgl. Sachverständigenrat zur Begutachtung der gesamtwirtschaftlichen Entwicklung, Vor Kurskorrekturen, Sondergutachten vom 4. Juli 1981, S. 9.

2) Ebenda, S. 9 .

3) Derselbe, Jahresgutachten 1975, a.a.0., Ziff. 228; anders gewendet heiBt dies, daB "steigende Defizite die Erwartungen höherer Zinsen wecken" und "die Furcht vor ... später ansteigenden Steuerlasten beflügeln". Derselbe, Jahresgutachten 1978/79, a.a.O., Ziff. 308 . 
beschleunigen" ${ }^{11}$ würden.

Steigende Preise machen zwar auf der einen Seite über zunehmende Verkaufserlöse mehr Investitionsausgaben rentabel; auch stellen sie für Konsumenten einen Anreiz dar, den Kauf von Verbrauchsgütern aus der zukunft in die Gegenwart zu verlagern. Auf der anderen Seite aber haben die Privaten die Erfahrung gemacht, daB ein zunehmendes Preisniveau auf mittlere sicht das wachstum schädigen, den Verteilungskampf verschärfen und in eine Stabilisierungskrise führen kann.

Werden die Erwartungen der Privaten hinsichtlich der Wirkungen eines finanzwirtschaftlichen demand management durch solche Erfahrungen geprägt, verschlechtern sich ihre Erwartungen in Bezug auf Gewinne und Haushaltseinkommen, wenn der Staat expansive Nachfrageimpulse entfajtet. Unter diesen Voraussetzungen ist "jede wohlbegründete Nachfragepolitik mit dem Risiko belastet, daB sie, in dem sie latente Inflationsfurcht mobilisiert, durch Verunsicherung von Erwartungen ganz oder teilweise verliert, was sie durch wohltätige Nachfrageimpulse an Konjunkturbelegung schafft".)

Der dadurch bewirkte Rückgang der realen Ausgaben der Privaten wird noch verstärkt, wenn die Furcht, an den Folgen des staatlichen demand management $z u$ verlieren, nicht nur die Nachfrager von Gütern (wegen schlechterer Erwartungen in Bezug auf Haushaltseinkommen und Gewinne) dazu veranlaBt, ihre Ausgaben einzuschränken, sondern auch die Produzenten (Unternehmer, Arbeitnehmer, Kapitalgeber) dazu animiert, ihre nominellen Ansprüche ( wegen steigender Preise und der Angst, an den steigenden Preisen zu verlieren) um mehr zu erhöhen, als nötig ist, um diese real zu halten. Dann

1) Sachverständigenrat zur Begutachtung der gesamtwirtschaftlichen Entwicklung, Jahresgutachten, 1975, a.a.0., Ziff. 228 .

2) Sievert, O., Die Steuerbarkeit der Konjunktur durch den staat, a.a.0., S. 816 . 
werden durch das finanzwirtschaftliche demand management nicht nur preiserwartungsinduzierte nachfrageseitige, sondern auch preiserwartungsinduzierte angebotsseitige Verdrängungseffekte bewirkt.

2. Finanzwirtschaftliches demand management und rationale Preiserwartungen

a) Konsequenzen rationaler Erwartungen

Bilden die Privaten rationale Erwartungen, sind sie genau darüber im Bilde, welche Folgen es hat, wenn der Staat stabilisierungspolitisch tätig wird. Sie kennen nämlich die struktur des ökonomischen Prozesses. Mithin kann der staat auf all die Transmissionswege finanzwirtschaftlicher Impulse nicht bauen, für die eine Illusion der Privaten notwendig ist.

Haben die Privaten rationale Erwartungen, unterliegen sie also erstens keiner Ricardo-Illusion. Sie wissen exakt, welche Steuerlasten auf sie infolge der Staatsverschuldung zukommen und auch, wie sich diese zeitlich verteilen. Wenn zudem die sonstigen Ricardo-Bedingungen erfüllt sind, entfallen folglich konventionelle Vermögenseffekte.

Es ist ihnen zweitens klar, daB in Höhe der Transformationsausgaben der staat investive und konsumtive öffentliche Leistungen an die Privaten abgibt. Bei den Privaten herrscht keine Fiskalillusion.

Letztlich sind sie auch daruber informiert, um welchen Betrag und mit welcher zeitlichen Verzögerung sich das preisniveau infolge des staatlichen Handelns erhöht. Sie sind folglich frei von Geldillusion. 
Mit der Annahme rationaler Preiserwartungen begründen die Rationalisten ihre These einer Ineffizienz jeglicher (geldpolitischer wie finanzpolitischer) Nachfragesteuerung, die einem bestimmten Handlungsmuster folgt. Einem bestimmten Handlungsmuster folgend heiBt, daB sich die politischen Akteure beim Treffen von MaBnahmen an eine bestimmte Handlungsmaxime halten, beispielsweise an die keynesianische Handlungsmaxime eines antizyklischen demand management, und daB die Verănderungen von Politikvariablen aus diesem Grund für dle Privaten antiziplerbar sind. Mit ihren therlegungen knüpfen die Rationalisten an die erstmals von Friedman und Phelps vorgetragene sog. Akzelerationsthese an, von der sogleich die Rede sein wird.

b) Die These der Rationalisten

aa) Die Friedman/Phelps-These und ihre Erweiterung durch die Rationalisten

In langer Frist, so Friedman und Phelps"' tendieren Beschäftigung und Produktion zu einem "natürlichen" Niveau, welches "durch reale Bedingungen bestimmt wird, die sich uber relative Preise und relative Kosten auswirken"2) und also von der gesamtwirtschaftlichen Güternachfrage unabhängig 1st. Finanzwirtschaftliche Nachfrageimpulse können mithin langfristig das Beschäftigungsniveau nicht steigern, son-

1) Vgl. Friedman, M., The Role of Monetary Policy, in: American Economic Review, 1968, S. 1 ff. sowie Phelps, E.S., Phillips Curves, Expectations of Inflation and Optimal Unemployment over Time, in: Economica, 1967, S. $254 \mathrm{ff.;}$ für einen Uberblick vgl. Shiller, R.J., Rational Expectations and the Dynamic Structure of Macroeconomic Models, a.a.0., s. 7 ff. und Kühn, B., Rationale Erwartungen und Wirtschaftspolitik, a.a.0., S. $13 \mathrm{ff}$.

2) Brunner, K., Falsche Leitbilder für die Politiker, in: Die Zeit, $\mathrm{Nr} .50,1978, \mathrm{~S} .30$. 
dern erschöpfen sich allenfalls in Preiseffekten. Kurzfristig mag es indessen Abweichungen zwischen der tatsächlichen und der natürlichen Beschäftigung geben und zwar immer dann, wenn die privaten Wirtschaftssubjekte das Preisniveau fehlerhaft prognostizieren und deshalb über die "realen Bedingungen" nicht hinreichend im Bilde sind.

Weil das Kalkül der Arbeitsnachfrager vom tatsächlichen, das der Arbeitsanbieter aber vom fur die Periode erwarteten Reallohn bestimmt werde, kommt es (so zum Beispiel die Begründung Friedmans"), Immer dann zu einer Zunahme der Beschäftigung über ihr natürliches Niveau hinaus, wenn das Preisniveau unterschätzt wird. Den Arbeitsanbietern erscheint in diesem Fall der Reallohn höher als er faktisch ist, und sie bieten bei jedem Nominallohn vergleichsweise $z u$ viel Arbeit an.

Die Beschäftigung ist folglich eine Abhängige der Differenz zwischen tatsächlichem und für die Periode erwartetem Preisniveau. Auf Dauer sind deshalb positive Beschäftigungseffekte nur dadurch zu erzielen, da $B$ das tatsächliche Preisniveau permanent über dem erwarteten liegt. Wenn die Wirtschaftssubjekte ihre Erwartungen autoregressiv bilden, was Phelps und Friedman unterstellt haben, ist eine dauerhafte Steigerung der Beschäftigung über ihr natürliches Niveau hinaus nur durch eine ständige Akzeleration der Geldentwertung zu erreichen (Akzelerationsthese).

1) Vgl. Friedman, M., The Role of Monetary Policy, a.a.O., S. 7 ff.; vgl. hierzu auch Gordon, R.J., Output Fluctuations and Gradual Price Adjustment, in: Journal of Economic Literature, 1981, S. 504 f. 
Formen dagegen die privaten Wirtschaftssubjekte ihre Erwartungen rational und treten keine zufälligen Ereignisse ein, kann es zu einer falschen Prognose des Preisniveaus und daraus resultierenden Beschäftigungseffekten gar nicht kommen. Was Friedman und Phelps als langfristiges Ergebnis ansehen, stellt sich nach Ansicht der Rationalisten schon in kurzer Frist ein ${ }^{1}$ ? Auch die kurzfristige Phillips-Kurve ist folglich eine Senkrechte.

\section{bb) Darstellung}

Die These der Rationalisten wird im folgenden anhand eines einfachen Gütermarkitmodells erläutert. Dieses ist durch alle Eigenschaften gekennzeichnet, auf die es Rationalisten ankommt, wenn sie ihre These von der Ineffizienz eines demand management begründen ${ }^{2}$ ! Die aus sicht der Rationalisten wesentlichen Charakteristika sind, daB erstens die Güterangebotshypothese eine vom Lucas-Typ ist und sich deshalb das Güterangebot nicht, wie im Grundmodell, völlig preiselastisch an die Güternachfrage anpaßt, sondern von Preiserwartungen dominiert wird, daB sich zweitens die Erwartungen rational bilden, daB drittens die finanzpolitischen Akteure bei der Ausgabengestaltung einem Handlungsmuster, das den Privaten bekannt ist, folgen und mithin

1) "If expectations are rationally based and if it is true that fiscal expansion will only raise prices and have no real effects, then this end could be achieved very much more quickly by anticipation". Argy, V., Some Notes on Fiscal Policy and Crowding Out, a.a.0., S. $67 \mathrm{f}$.

2) Vgl. für einen Uberblick: Ramser, H.J., Rationale Erwartungen und Wirtschaftspolitik, in: Zeitschrift für die gesamte Staatswissenschaft, 1978, S. 59 ff.; Shiller, R.J., Rational Expectations and the Dynamic Structure of Macroeconomic Models, a.a.O., S. 8 ff.; Neumann, M.J. M. Rationale Erwartungen in Makromodellen, a.a.O., S. 375 ff.: Kühn, B., Rationale Erwartungen und Wirtschaftspolitik, a.a.O., S. $43 \mathrm{ff.;}$ Klausinger, H., Rationale Erwartungen und die Theorie der Stabilisierungspolitik, a.a.0., S. $63 \mathrm{ff.;} \mathrm{McCallum,} \mathrm{B.,} \mathrm{Rational} \mathrm{Expectations}$ and Macroeconomic Stabilization Policy, a.a.O., S. 719 ff. 
die Privaten von den staatlichen Handlungen nicht überrascht werden können sowie daB viertens die Preise ausreichend flexibel sind. Das Modell besteht in seiner strukturellen Form aus fünf Gleichungen (vgl. Ubersicht 11) 1 !

Ubersicht 11: Ein einfaches Gütermarktmodell mit rationalen Erwartungen in seiner strukturellen Form

$$
\begin{aligned}
& (2-5-6) \log Y^{s r}=\log Y^{d r} \\
& (2-5-7) \log Y^{r}=\log Y^{s r}=\log Y^{d r}, \\
& (2-5-8) \log Y^{S r}=\log \bar{Y}^{n r}+a\left(\log P-e_{-1} \log P\right)+\log u \\
& \text { mit } \mathbf{a}>0 \\
& (2-5-9) \quad \log Y^{d r}=b(\log \bar{M}-\log P)+\log G^{r} \\
& \text { mit } b>0 \\
& (2-5-10) \log G^{r}=g\left(\log Y_{-1}^{r}-\log \bar{Y}^{n r}\right) \\
& \text { mit } g<0
\end{aligned}
$$

Gleichung $(2-5-6)$ ist die Gleichgewichtsbedingung für den Gütermarkt.

GemäB Gleichung (2-5-7) stimmt das gleichgewichtige Sozialprodukt mit dem tatsächlichen überein.

1) Rationalisten verwenden zur analytischen Vereinfachung zumeist Modelle, in denen die Variablen logarithmiert sind und eine lineare Beziehung zwischen den logarithmierten Variablen besteht. 
Gleichung (2-5-8). gibt eine Angebotsbeziehung vom Lucas-Typ wider ${ }^{1}$. Demnach entspricht das reale Güterangebot $\left(\log \mathrm{Y}^{\mathrm{Sr}}\right)$ seinem natürlichen Niveau $\left(\log \overline{\mathrm{Y}}^{\mathrm{nr}}\right)$, wenn nur keine zufälligen Angebotsschocks (log u) auftreten und wenn das tatsächliche Preisniveau ( $\log P)$ gleich dem am Ende der Vorperiode gebildeten erwarteten Preisniveau $\left(e_{-1} \log P\right)$ ist. Besteht indessen eine Differenz, varilert das reale Güterangebot nach MaBgabe von a .

Die reale Güternachfrage $\left(\log Y^{d r}\right)$ ist gemäB Gleichung (2-5-9) von der realen Kasse $[b(\log \bar{M}-\log P)]$ sowie von den realen Transformationsausgaben $\left(\log \mathrm{G}^{\mathrm{r}}\right)$ abhängig.

Gleichung (2-5-10) beschreibt die Handlungsmaxime, der die finanzpolitischen Akteure folgen ${ }^{2)}$. Auf Abweichungen des tatsächlichen Sozialprodukts von seinem natürlichen Niveau $\left(\log Y_{-1}-\log \overline{\mathrm{Y}}^{\mathrm{nr}}\right)$ reagiert der staat in der Folgeperiode mit einer gegenläufigen Veränderung seiner realen Transformationsausgaben. Die finanzpolitischen Akteure lassen sich also von der keynesianischen Handlungsmaxime eines antizyklischen demand management leiten, wenn sie stabilisierungspolitisch tätig werden.

1) Kennzeichen solcher Güterangebotsfunktionen ist, daB Divergenzen zwischen tatsächlichen und erwarteten Preisen Determinanten des Güterangebots sind. Die Angebotshypothese $(2-5-8)$ ist die Sargent/Wallace-Variante. Für einen Uberblick weiterer in der Literatur gebräuchlicher Formulierungen vgl. Minford, P., Peel, D., The Role of Monetary Stabilization Policy under Rational Expectations, In: The Manchester School, 1981, S. $40 \mathrm{ff}$.

2) Rationalisten vernachlässigen regelmäBig finanzwirtschaftliche Aktivitäten und beschränken ihre Wirkungsanalyse meistens auf eine Geldmengenpolitik; Ausnahmen sind Shiller, R.J., Rational Expectations and the Dynamic Structure of Macroeconomic Models, a.a.0., S. $10 \mathrm{ff}$.; McCallum, B.T., Whitaker, J.K., The Effectiveness of Fiscal Feedback Rules and Automatic Stabilizers under Rational Expectations, in: Journal of Monetary Economics, 1979, S. $171 \mathrm{ff.;} \mathrm{Kühn,} \mathrm{B.,} \mathrm{Rationale} \mathrm{Erwartungen} \mathrm{und}$ Wirtschaftspolitik, a.a.0., S. $65 \mathrm{ff.;} \mathrm{Klausinger,} \mathrm{K.,}$ Rationale Erwartungen und die Theorie der Stabilisierungspolitik, a.a.0., S. $63 \mathrm{ff}$. 
Um das Modell zu lösen, muB als Vorarbeit noch der erwartete Wert für das Preisniveau (e $\left.e_{-1} \log P\right)$ ermittelt werden. Da die Wirtschaftssubjekte beim Bilden von Erwartungen rational vorgehen, kennen sie das Modell, in dem sie agieren, und sie wissen, daB. sich die Preise solange verändern, bis sich der Gütermarkt im Gleichgewicht befindet. Ihre Preiserwartungen basieren folglich auf der aus den Gleichungen $(2-5-6),(2-5-8)$ und $(2-5-9)$ abgeleiteten semi-reduzierten Gleichung für das Preisniveau:

$$
\begin{aligned}
& (2-5-11) \quad \log P=\frac{1}{a+b}\left(b \log \bar{M}+\log G^{r}-\log \bar{Y}^{n r}\right. \\
& -\log u+a e_{-1} \log \text { P). }
\end{aligned}
$$

Der Erwartungswert und mithin die rationalen Erwartungen der Privaten für das preisniveau $\left(e_{-1} \log\right.$ P) lauten dann:

$(2-5-12) \quad e_{-1} \log P=\log \bar{M}+\frac{1}{b} e_{-1} \log G^{r}-\frac{1}{b} \bar{Y}^{n r}$.

In welcher Hohe die Privaten und der staat in der betrachteten Periode reale Güternachfrage entfalten, kann nun aus Gleichung (2-5-13) abgelesen werden, die aus den Gleichungen $(2-5-6)$ bis $(2-5-9)$ und $(2-5-12)$ folgt:

$$
\begin{aligned}
(2-5-13) \log Y^{r}= & \log \bar{Y}^{n r}+\frac{a}{a+b}\left(\log G^{r}-e_{-1} \log G^{r}\right) \\
& +\frac{b}{a+b} \log u .
\end{aligned}
$$

Von den Bestimmungsfaktoren der realen Gesamtausgaben ist für unsere Fragestellung der Klammerausdruck wichtig: Die realen Transformationsausgaben beeinflussen immer dann die gesamtwirtschaftliche reale Güternachfrage, wenn ihr tatsächlicher Betrag von dem abweicht, welchen die Privaten erwartet haben. Im vorliegenden Modell kann dies nicht vorkommen. Die privaten Wirtschaftssub- 
jekte sind nämlich "zu gut" informiert. Anhand der Regel (2-5-10) prognostizieren sie exakt die vom Staat entfaltete reale Guternachfrage " Folglich sind die tatsächlich vom staat getätigten und die von den Privaten erwarteten realen Transformationsausgaben identisch:

$$
(2-5-14) \log G^{r}=e_{-1} \log G^{r},
$$

und Gleichung (2-5-13) geht in

$$
(2-5-15) \log Y^{r}=\log \bar{Y}^{n r}+\frac{b}{a+b} \log u
$$

über. Das reale Sozialprodukt wird von finanzwirtschaftlichen Nachfrageimpulsen, die einer bestimmten Handlungsmaxime folgen, nicht beeinfluBt. Sind die Impulse expansiv - weil in der Vorperiode das tatsächliche Sozialprodukt unter sein natürliches Niveau gesunken war - kommt es schon in kurzer Frist zu einem vollständigen realen crowding-out.

cc) Kritik

Die These der Rationalisten ist heftig kritisiert worden. Die dabei vorgetragenen Einwendungen betreffen vor allem zwei Annahmen der Rationalisten, zum einen, daB die Privaten ihre rationalen Erwartungen auf der Basis eines umfassenden Informationsstandes bilden und zum anderen, daB die Preise

1) "Viele 'Spieler im wirtschaftsprozeB' finden es vorteilhaft, systematisch die künftige wirtschafts- und vor allem makropolitische Linie zu beurteilen. Diese Beurteilung beeinfluBt die Inflationserwartungen weitgehend. So ergibt sich, daB Veränderungen des finanzpolitischen Kurses Inflationserwartungen modifiziert, bevor die laufende Inflationsrate unmittelbar betroffen wird". Brunner, K., Falsche Leitbilder für die Politiker, a.a.0., s. 30 . 
hinreichend flexibel sind.

Zum ersten Einwand: Die Hypothese der Rationalisten, die privaten wirtschaftssubjekte seien umfassend informiert, wenn sie Erwartungen bilden, ist zweifellos unrealistisch ${ }^{1}$. Denn in der Wirklichkeit verursacht die Beschaffung und Verarbeitung von Informationen Kosten in Form von Zeit und realen Ressourcen. Mithin ist es nicht sinnvoll, den SuchprozeB solange fortzusetzen, bis der Ignoranzgrad den Wert Null angenommen hat. Vielmehr ist er dann abzubrechen, wenn der Grenzgewinn zusätzlicher Informationen gleich $\mathrm{Null}$ wird ${ }^{2}$ !

Streben die privaten Wirtschaftssubjekte keine maximale, sondern eine optimale Informiertheit an, können verzerrte Erwartungen ihr Verhalten bestimmen. Unterschätzen sie deshalb den Preiseffekt eines finanzwirtschaftlichen Nachfrageimpulses, ist ein positiver Mengeneffekt zu verzeichnen. Sofern dieser geringer als die zusätzlichen realen Transformationsausgaben ausfällt, ändert sich jedoch nicht die Qualität, sondern nur die Quantität des durch die staatliche Intervention bewirkten Privatausgabeneffektes; das crowding-out ist nicht mehr vollständig, sondern nur noch partiell. Uberschätzen die Privaten jedoch den Preiseffekt, kommt es zu einem übermäBigen crowding-out. Die realen Ausgaben der Privaten gehen um mehr zurück als die des staates steigen.

Zum zweiten Einwand: Die postulierte Flexibilität der Preise kollidiert ebenfalls mit Beobachtungen in der

1) Vgl. Shiller, R.J., Rational Expectations and the Dynamic Structure of Macroeconomic Models, a.a.0., S. 33 ff.; Ramser, H.J., Rationale Erwartungen und Wirtschaftspolitik, a.a.O., S. 62 ff.; Neumann, M.J.M., Rationale Erwartungen in Makromodellen, a.a.O., S. 386 ff.: McCallum, B.T., Rational Expectations and Macroeconomic Stabilization Policy, a.a.0., S. $736 \mathrm{ff}$.

2) Dann können auch mittels Daumenregeln oder autoregressiver Verfahren rationale Erwartungen gebildet werden. 
Wirklichkeit") Die Preise reagieren nicht so rasch auf eine Zunahme der gesamtwirtschaftlichen Güternachfrage, wie die Rationalisten annehmen. Eine ökonomische Erklärung für inflexible Preise liefern neuere Ansätze, welche in der Literatur unter dem Namen Kontrakttheorie zusammengefaBt werden.' Dort wird gezeigt, daB es im Interesse der privaten Wirtschaftssubjekte sein kann, sich auf längere Frist vertraglich $\mathrm{zu}$ binden. Schnelle Preisanpassungen sind dann nicht mehr möglich.

Diese Erwägungen sind von den Rationalisten aufgegriffen und in ihre Modellanalysen integriert worden ${ }^{3)}$. Die Schlubfolgerung eines vollständigen realen crowding-out in der kurzen Frist ist bei Existenz vertragsmäBig bedingter Preisrigiditäten nicht mehr haltbar. Es können positive Beschäftigungseffekte erzielt werden, und es kann dadurch das AusmaB der Verdrängung der realen Güternachfrage der Privaten abgeschwächt werden.

1) Vgl. Ramser, H.J., Rationale Erwartungen und Wirtschaftspolitik, a.a.0., S. 67 ff.; McCal.um, B.T., Rational. Expectations and Macroeconomic Stabilization Policy, a.a.0., S. $729 \mathrm{ff.;}$ Gordon, R.J., Output Fluctuations and Gradual Price Adjustment, a.a.O., S. $511 \mathrm{ff}$.

2) Für einen Uberblick vgl. Kühn, B., Rationale Erwartungen und Wirtschaftspolitik, a.a.0., s. 23 ff. sowie Klausinger, H., Rationale Erwartungen und die Theorie der Stabilisierungspolitik, a.a.0., S. $39 \mathrm{ff}$.

3) Für einen tberblick vgl. Kühn, B., Rationale Erwartungen und Wirtschaftspolitik, a.a.0., s. $84 \mathrm{ff}$. sowie Klausinger, H., Rationale Erwartungen und die Theorie der Stabilisierungspolitik, a.a.0., S. $113 \mathrm{ff}$. 
Sechstes Kapitel: Resümee

In unserer Untersuchung ging es um die These der Monetaristen, die Finanzpolitik könne bei Unterbeschäftigung keine stabilisierungspolitische Aufgabe wahrnehmen. Denn nach monetaristischer Meinung führen staatliche Ankurbelungsmaßnahmen lediglich zu einem crowding-out, also im wesentlichen nur zu einer Veränderung der struktur und nicht des Niveaus der gesamtwirtschaftlichen Ausgaben. Die Argumente, die zur Stützung der monetaristischen These vorgebracht werden, wurden dargestellt und auf ihre plausibilität hin überprüft.

Dabei stand für das finanzwirtschaftliche Handeln eine kreditär finanzierte Erhöhung der Transformationsausgaben. Die Geldmenge blieb im Zuge dessen konstant - abgesehen von dem Abschnitt, in welchem es um Preiseffekte aufgrund eines finanzwirtschaftlich verursachten Nachfragesogs ging;dort verfolgten die geldpolitischen Akteure keine Strategie der Geldmengenfixierung, sondern eine Strategie der zinsfixierung. Betrachtet wurde ein kurz- und mittelfristiger Zeitraum, zu dessen Beginn die Produktionsfaktoren unterbeschäftigt waren. Daß Unterbeschäftigung nicht nur durch einen Güternachfragemangel (nachfrageseitige Störimpulse), sondern auch durch den Verteilungsstreit (angebotsseitige störimpulse) verursacht sein kann, spielte für die Begründung eines crowding-out nur zweimal eine Rolle: Bei angebotsseitigen Störimpulsen entfällt zum einen der Akzelerationseffekt, und zum anderen verpuffen finanzwirtschaftliche Nachfrageimpulse in einem Preisschub, wenn sie auf ein unbewegliches Güterangebot treffen. Deshalb wurde, abgesehen davon, unterstellt, die Gründe für die Unterbeschäftigung lägen auf der Nachfrageseite des Gütermarktes - wir hatten es also 
meistens mit keynesianischen Problemen zu tun, zu denen die keynesianische Rezeptur prima vista paBt.

Die Transmissionskanäle, über die ein finanzwirtschaftliches demand management EinfluB auf die Ausgaben der Privaten nehmen kann, lassen sich zu fünf Gruppen zusammenfassen. Davon sind fur ein crowding-out nicht nur jene wichtig, über welche die Ausgabentătigkeit der Privaten gedämpft wird, sondern auch jene, ubber die sie stimuliert wird. Denn fallen die Anreizeffekte schwach aus, kann damit ebenso wie mit Verdrängungseffekten begründet werden, daB die private Güternachfrage unter das Niveau sinkt, welches entfaltet worden wäre, hätte der staat nicht stabilisierungspolitische Maßnahmen ergriffen.

Es sinken, erstens, die privaten zinsabhängigen Ausgaben um den Betrag des Hicksschen crowding-out - und zwar um so mehr, je elastischer die privaten Güternachfrager und je unelastischer die Geldnachfrager auf eine Zinserhöhung reagieren. Nach einer Untersuchung von B. Friedman beläuft sich der Effizienzverlust aufgrund des Hicksschen crowding-out auf etwa 10 vom Hundert.

Zweitens nimmt nach monetaristischer Meinung infolge einer Plazierung von Staatspapieren in den Portefeuilles der Privaten die Geldnachfrage und deswegen das $\mathrm{Zinsniveau} \mathrm{zu}$, wodurch die $z$ insabhängigen Ausgaben der Privaten verdrängt werden (konventionelles Portfolio-crowding-out). Der konventionelle Portfolio-crowding-out-Effekt ist vor allem auf mittlere Frist wichtig. Denn wie alle Vermögenseffekte ist auch der konventionelle Portfolio-crowding-out-Effekt ein BestandsgröBeneffekt. Er wirkt deshalb kumulativ: Erhöht der staat seine Güternachfrage auf Dauer, muB er solange, wie der staatshaushalt nicht wieder ausgeglichen ist, in jeder Haushaltsperiode erneut staatspapiere in den Portefeuilles der Privaten unterbringen, wodurch die Pri- 
vatausgaben ständig weiter zurückgedrängt werden.

Der konventionelle Portfolio-crowding-out-Effekt ist um so stärker, (a) je mehr im Zuge der Emission von Staatspapieren die Geldnachfrage steigt, (b) je weniger Risikoerwägungen bei der Diversifizierung des Vermögens auf die Portfoliokomponenten eine Rolle splelen, (c) je weniger Gewicht die steuerverpflichtungen, welche infolge der Staatsverschuldung auf die Privaten zukommen, bei der Strukturierung des Portefeuilles haben (tax discounting) und (d) je weniger sich der Umfang des Portefeuilles der Privaten infolge des staatlichen Handelns vergröBert. Zudem wird uno actu mit dem konventionellen Portfolioeffekt ein (expansiver) Lerner-Effekt bewirkt.

Im Streit zwischen Fontänentheoretikern und Quellentheoretikern um die adäquate theoretische Vorstellung zur Abschätzung einer Verdrängungskonkurrenz zwischen Staat und Privaten auf dem Kapitalmarkt sprechen das Hickssche crowding-out und das konventionelle Portfolio-crowdingout gegen die Meinung der Fontänentheoretiker. Allgemein: Fontänentheoretiker gehen implizite davon aus, daB das staatliche Handeln aggregativ effizient ist.

Neben den konventionellen Vermögenseffekten können durch das finanzwirtschaftliche Handeln noch eine Reihe anderer Vermögensmengeneffekte und auch Vermögenswerteffekte bewirkt werden. Uber diese Wege ubt der Staat sowohl einen stimulierenden als auch einen die privaten Ausgaben dämpfenden EinfluB aus. Der Gesamteffekt ist nur schwer abschätzbar.

Drittens wird die (reale) Güternachfrage der Privaten durch steigende Preise zurückgedrängt. $\mathrm{Zu}$ steigenden Preisen kann es infolge des staatlichen Handelns auf zwei Wegen kommen, durch einen Nachfragesog und durch einen Angebotsdruck. 
Damit durch finanzwirtschaftliches Handeln ein Nachfragesog entsteht und dieser sich in Preiseffekten - trotz Unterbeschäftigung - niederschlägt, muB allerdings (a) im monetären Bereich der Geldstrom zunehmen und (b) auf dem Gütermarkt der Nachfragesog auf eine nicht ausdehnungsfähige Produktion treffen. Die Erfüllung der Bedingung (a) wird erschwert, wenn die Notenbank eine Geldmengenpolitik betreibt. Die zweitgenannte Bedingung ist dann erfullt, wenn die Ursachen für die Unterbeschäftigung auf der Angebotsseite liegen oder wenn dort, wo der staat zusätzliche Güternachfrage entfaltet, die Produktionsfaktoren vollbeschäftigt sind, obgleich allgemein ein Güternachfragemangel besteht. Zur Zeit liegen in der Bundesrepublik Deutschland die Ursachen für die Unterbeschäftigung u.E. eher auf der Angebotsseite; zudem haben sich die öffentlichen Ausgabenprogramme in der Vergangenheit regelmäBig auf den Baumarkt konzentriert. Dennoch scheint das Risiko von Preiseffekten aufgrund eines finanzwirtschaftlich verursachten Nachfragesogs nicht allzu groB zu sein, weil die Bundesbank eine an der Geldmenge orientierte Politik betreibt. zu befürchten sind dagegen Preiseffekte aufgrund eines finanzwirtschaftlich verursachten Angebotsdrucks.

Ein Angebotsdruck wird durch das finanzwirtschaftliche demand management immer dann ausgelöst, wenn das staatliche Handeln diejenigen, welche Anspruch auf das Produktionsergebnis erheben, dazu veranlaBt, ihre Ansprüche $z u$ erhöhen.

So können Kapitalgeber einen höheren Zins fordern (weil sich aufgrund des staatlichen Engagements die Knappheitsrelationen auf dem Finanzmarkt verschieben) und Unternehmer ihre Risikoprämie aufstocken (als Ausgleich dafür, daB sie sich einer gröBeren Unsicherheit darüber ausgesetzt wähnen, in welchem AusmaB in der zukunft mit ähnlichen staatlichen Aktionen zu rechnen ist). 
Sofern das finanzwirtschaftiche demand management ein Mittel zur Erfullung einer Vollbeschäftigungsgarantie ist, brauchen die Arbeitnehmer bei ihren Lohnverhandlungen aus $\mathrm{zu}$ hohen Lohnabschlussen resultierende Beschäftigungseffekte nicht $z$ u beachten. Denn sie können darauf vertrauen, daB der Staat bei einer sich abzeichnenden Unterbeschäftigungstendenz expansive MaBnahmen ergreift, um sein abgegebenes Versprechen $z u$ erfüllen. Das finanzwirtschaftliche demand management verleitet mithin die Arbeitnehmer dazu, höhere als mit stabilem Geldwert vereinbare Lohnzuwächse auszuhandeln. In der Bundesrepublik Deutschland dürfte in der letzten Dekade diese Ursache für ein crowding-out keine unwesentliche Rolle gespielt haben.

Viertens sind Erwartungseffekte von Bedeutung. Nach Meinung der Rationalisten entfällt für die finanzpolitischen Akteure die Möglichkeit, mit einem demand management, welches einem bestimmten Handlungsmuster folgt, $\mathrm{Be}-$ schäftigungseffekte $z u$ erzielen. Auch die kurzfristige Phillips-Kurve ist nach Ansicht der Rationalisten eine Senkrechte.

Wichtiger dürfte allerdings sein, daB psychologische Faktoren bei der Erwartungsbildung - gerade im Zusammenhang mit der Staatsverschuldung - Gewicht haben und auch, was der Sachverständigenrat betont, die mit dem demand management gemachten Erfahrungen. Worauf es bei letzterem ankommt, sind in der Bundesrepublik Deutschland zwe 1 währungsreformen und in jüngster Zeit vergleichsweise hohe Geldentwertungsraten. Dies zusammen scheint im Augenblick in der Bundesrepublik Deutschland die Folge zu haben, daB die Privaten zukunftsvertrauen verlieren, wenn der staat aktiv wird und sie deshalb ihre Ausgaben einschrănken. 
Eine Stimulierung der Ausgabenaktivitäten der Privaten muB hauptsächlich von der Staatsausgabenseite ausgehen, also, fünftes, durch den direkten staatsausgabeneffekt, den einkommensinduzierten Konsumeffekt und den Akzelerationseffekt erfolgen.

Mit auf dem Kreditmarkt beschafften finanziellen Mitteln wird insoweit kein direkter staatsausgabeneffekt bewirkt, wie die vereinnahmten Gelder zum Tätigen von Personalausgaben oder zur Begleichung von durch die staatliche Kreditaufnahme entstandenen $\mathrm{Z}$ insforderungen der Privaten verwendet werden. Der zweitgenannte Gesichtspunkt ist insbesondere auf mittlere Frist (und derzeit in der Bundesrepublik Deutschland) wichtig. Denn die Güternachfrage, die der staat auf Dauer mit dauerhaft erhöhten kreditär finanzierten Transformationsausgaben entfalten kann, wird von Haushaltsperiode zu Haushaltsperiode kleiner, weil die Zinszahlungen einen immer gröBer werdenden Anteil einnehmen.

Der einkommensinduzierte Konsumeffekt kann aus zwei Gründen abgeschwächt werden. Orientieren sich die privaten Konsumenten bei ihren Ausgabedispositionen am permanenten Einkommen (was die Finanzpolitik hierzulande zum Beispiel 1970/72 beim "Konjunkturzuschlag" erfahren mußte), reagieren sie auf zeitlich befristete finanzwirtschaftliche Nachfrageimpulse nur sehr träge. Unterliegen die Privaten keiner Fiskalillusion und ziehen sie deshalb die vom staat erstellten öffentlichen Leistungen bei ihren Ausgabeplänen mit ins Kalkül, fällt der einkommensinduzierte Konsumeffekt insoweit schwächer aus, wie die privaten Haushalte von der staatlichen Leistungsabgabe profitieren und deshalb ihre privaten Konsumausgaben einschränken. 
Auch David und Scadding gehen davon aus, daB die Privaten frei von Fiskalillusion sind. Zudem nehmen sie an, daB (a) die Privaten von der Finanzierungsform staatlicher Güternachfrage auf die Art der erstellten öffentlichen Leistungen schlieBen, daB (b) die Privaten eine bestimmte gesamtwirtschaftliche Relation zwischen investiver und konsumtiver Verwendung der insgesamt zur Verfügung stehenden Ressourcen wünschen und daB (c) es ihnen gleichgültig ist, welchen Anteil daran jeweils staat und Private haben. Aus diesen Annahmen folgern David und Scadding, daB staatliches Handeln generell ein vollständiges ex ante crowding-out bewirkt.

Mit einem Akzelerationseffekt ist dann nicht zu rechnen, wenn (was unseres Erachtens zur Zeit in der Bundesrepublik Deutschland zutrifft) die Ursachen für das Unterlassen von Investitionen auf der Angebotsseite liegen.

Als Ergebnis unserer Untersuchung halten wir fest: Es gibt eine Vielzahl von Argumenten, mit denen die monetaristische These begründet werden kann, daB die beabsichtigte Beschäftigungswirkung ausbleibt, wenn der Staat expansive Nachfrageimpulse entfaltet. Allerdings hat sich gezeigt, daB eine Reihe dieser Argumente keine generelle Gültigkeit hat, sondern an die Erfüllung bestimmter Bedingungen geknüpft ist. So kommt es zum Beispiel nur dann zu keinem Akzelerationseffekt, wenn die Investitionszurückhaltung angebotsseitig bedingt ist, und die These des Sachverständigenrates, Erwartungseffekte wïrden $z u$ einem crowding-out führen, gilt nicht generell, sondern nur dann, wenn die Privaten in der Vergangenheit schlechte Erfahrungen mit einem finanzwirtschaftlichen demand management gemacht haben. Deshalb ist nicht situationsneutral zu entscheiden, inwieweit der Pessimismus der Monetaristen berechtigt ist. Die Erörterung der Argumente, die zur stützung der crowding-out-These vorgebracht werden, hat zumindest deutlich gemacht, daB von 
den beiden möglichen Resultaten einer Finanzpolitik keynesianischer Provenienz - crowding-out oder pullingin - das erstgenannte nicht als akademisch zwar interessanter, aber unrealistischer spezialfall abgetan werden darf (was Keynesianer machen), sondern nicht selten - wie U.E. zum Beispiel zur zeit in der Bundesrepublik Deutschland - das wahrscheinlichere der beiden möglichen Ergebnisse ist.

Noch fragwürdiger wird eine staatliche Nachfragesteuerung, wenn die stabilisierungspolitische Unproduktivität von einer wachstumspolitischen Kontraproduktivität ${ }^{1)}$ begleitet wird. Weil in diesem Fall "die Programme zu stark am Konsum und $\mathrm{zu}$ wenig an Investitionen orientiert sind"2) hat eine Finanzpolitik keynesianischer Provenienz dann nicht nur gegen sich, daB keine stabilisierungspolitischen Erfolge erzielt werden, sondern auch, daB mit jeder staatlichen Aktivität das wachstumsiel weiter in die Ferne rückt.

1) Vgl. Sundararajan, V., Thakur, S., Public Investment, Crowding Out, and Growth: A Dynamic Model Applied to India and Korea, in: Staff Papers, International Monetary Fund, 1980, S. 814 ff.; Fürstenberg, G.M. von, The Effect of Changing Size and Composition of Government Purchases on Potential Output, in: The Review of Economic and Statistics, 1980, S. 74 ff.; für eine Uberblick zum Zusammenhang zwischen finanzpolitischen MaBnahmen und privater Realkapitalbildung vgl. Fürstenberg, G.M. von, Malkiel, B., The Government and Capital Formation: A Survey of Recent Issues, in: Journal of Economic Literature, 1977, S. 835 ff. sowie Schmidt, K., Finanzpolitik für mehr wirtschaftliches Wachstum, in: Bohley, P., Tolkemitt, G. (Hrsg.), Wirtschaftswissenschaft als Grundlage staatlichen Handelns, Tübingen, 1979, S. 115 ff.

2) Schmidt, K., Diskussionsbeitrag, in: Beihefte der Konjunkturpolitik, Heft 27, 1980 , Shiller-978-3-631-75197-8 
SYMBOLVERZEICHNIS

A

AB

AZ

B

${ }^{d}$

$\mathrm{B}^{\mathrm{S}}$

C

c

$D$

$\mathrm{E}^{\mathrm{B}}$

$E^{I}$

$\mathrm{E}^{\mathrm{K}}$

$E^{M Z}$

e

G

$\mathrm{G}^{\mathrm{C}}$

$\mathrm{G}^{\mathrm{I}}$

I

i

ib

ik

K

$\mathrm{k}^{\mathrm{d}}$

$\mathrm{K}^{\mathrm{S}}$

L

$\log$

M

MZ

$M Z^{S}$
Erwerbspersonen

beschäftigte Arbeitskräfte

Arbeitszeit

Bestand an Staatsschuldtiteln bei den Privaten

Nachfrage nach Staatsschuldtiteln

Angebot an Staatsschuldtiteln

private Konsumausgabe

marginale Konsumquote

staatlicher Finanzierungssaldo

Erträge aus dem Halten von Staatsschuldtiteln

Erträge aus dem Halten von noch $\mathrm{zu}$ produzierendem Realkapital

Erträge aus dem Halten von

bestehendem Realkapital

Erträge aus dem Halten von Zentralbankgeld

Erwartungswert

Transformationsausgaben

staatlicher Konsum

staatliche Investitionen

private Investitionsausgaben

zins

Zins auf Staatsschuldtiteln

Zins auf bestehendes Realkapital

bestehendes Realkapital

Nachfrage nach bestehendem Realkapital

Angebot an bestehendem Realkapital

Geldnachfrage

Logarithmus

Geldmenge

Zentralbankgeldmenge

Zentralbankgeldangebot 


\begin{tabular}{|c|c|}
\hline $\mathbf{P}$ & Preisniveau \\
\hline $\mathrm{P}^{\mathrm{I}}$ & $\begin{array}{l}\text { Wiederbeschaffungskosten des Realkapitals, } \\
\text { Preis pro Einheit noch zu produzierendes } \\
\text { Realkapital }\end{array}$ \\
\hline $\mathrm{P}^{\mathrm{K}}$ & $\begin{array}{l}\text { Marktwert des Realkapitals, Preis pro } \\
\text { Einheit bereits existierendes Realkapital }\end{array}$ \\
\hline $\mathbf{r}$ & real (Superskript) \\
\hline $\mathbf{S}$ & privates Sparen \\
\hline $\mathbf{T}$ & (Netto-) Steuern $\left(=T^{b}-T R\right)$ \\
\hline $\mathrm{T}^{\mathrm{b}}$ & (Brutto-) Steuern \\
\hline$T^{B}$ & $\begin{array}{l}\text { Barwert der künftigen steuern zur Ver- } \\
\text { zinsung und Tilgung der Staatsschulden }\end{array}$ \\
\hline$t$ & marginaler Steuersatz \\
\hline TR & Transferzahlungen \\
\hline U & Umlaufsgeschwindigkeit des Geldes \\
\hline $\mathrm{V}$ & privates Vermögen \\
\hline$w^{C+I}$ & Wachstumsrate der privaten Ausgaben \\
\hline $\mathbf{Y}$ & tatsächliches sozialprodukt \\
\hline $\mathrm{Y}^{\mathrm{B}}$ & staatliche Zinszahlungen \\
\hline $\mathrm{Y}^{\mathrm{d}}$ & gesamtwirtschaftliche Güternachfrage \\
\hline $\mathrm{Y}^{\mathrm{n}}$ & "natürliches" Sozialprodukt \\
\hline $\mathrm{Y}^{\mathrm{P}}$ & Produktionspotential \\
\hline $\mathrm{Y}^{\mathbf{S}}$ & gesamtwirtschaftliches Güterangebot \\
\hline $\mathrm{Y}^{\mathrm{V}}$ & verfügbares Einkommen \\
\hline $\mathbf{Z}$ & $\begin{array}{l}\text { Bestand an staatsschuldtiteln bei der } \\
\text { Zentralbank }\end{array}$ \\
\hline
\end{tabular}


VERZEICHNIS DER UBERSICHTEN

Seite

Ubersicht 1: Kurzfristiges, mittelfristiges und langfristiges crowding-out

Ubersicht 2: Partielles, vollständiges und übermäßiges crowding-out

Ubersicht 3: Politikvarianten

Ubersicht 4: Abgrenzung von Geld- und Finanzpolitik nach Bent Hansen

Ubersicht 5: Das Grundmodell in seiner strukturellen Form

Ubersicht 6: Das erweiterte makroökonomische Portfoliomodell in seiner strukturellen Form

Ubersicht 7: Ursachen und Transmissionskanäle von Vermögenseffekten

Ubersicht 8: Geldvermögenskomponenten

tbersicht 9: Vermögenseffekte und private Ausgaben

Ubersicht 10: Die Staatsausgaben im Güterkreislauf

Ubersicht 11: Ein einfaches Gütermarktmodell mit rationalen Erwartungen in seiner strukturellen Form 
VERZEICHNIS DER ABBILDUNGEN

Seite

Abbildung 1: Gesamtausgabeneffekt und Privatausgabeneffekt im Grundmodell

Abbildung 2: Konventionelles Portfoliocrowding-out in der Entstehungsphase des Defizits

Abbildung 3: Konventionelles Portfoliocrowding-out in der reinen Finanzierungsphase des Defizits

Abbildung 4: Lerner-Effekt und konventioneller Portfolio-crowding-out-Effekt in der Entstehungsphase des Defizits

Abbildung 5: Lerner-Effekt und konventioneller Portfolio-crowding-out-Effekt in der reinen Finanzierungsphase des Defizits

Abbildung 6: Preis-Mengen-Diagramm mit "monetaristischer" und "keynesianischer" Güterangebotsfunktion

Abbildung 7: Staat]iche Beschäftigungsgarantie und Lohnansprüche 


\author{
Abrahams, B.A., \\ Schitz, M.D., \\ Andersen, L.C., \\ Jordan, J.L.,
}

Alexander, V., Brückmann, F. ,

Ando, A., Shell, K.,

Arcelli, M., Valiani, R.,

Arestis, P.,

Argy, V.,

Argy, V. ,

Artis, M.J.,

Bailey, M.J.,

Baird, C.W.,
The "Crowding-Out" Effect of Governmental Transfers on Private Charitable Contributions, in: Public Finance, 1978, S. 29 ff.

Monetary and Fiscal Actions: A Test of Their Relative Importance in Economic Stabilization, in: Federal Reserve Bank of St. Louis Review, November 1968, S. 11 ff.

Crowding-out-Effekte kommunaler Schuldenpolitik in der Bundesrepublik Deutschland, in:

Zeitschrift für Wirtschafts- und Sozialwissenschaften, 1978, S. $211 \mathrm{ff}$.

Appendix zu Ando, A., Modigliani, F., Some Reflections on Describing Structures of Financial Sectors, in: Fromm, G., Klein, L.R. (Hrsg.), The Brookings Model: Perspective and Recent Developments, Amsterdam, 1975, S. $560 \mathrm{ff}$.

Crowding Out: Some Reflections on Economic Policy, in: Review of the Economic Conditions in Italy, No 2 1979, S. 189 ff.

The "Crowding-Out" of Private Expenditures by Fiscal Actions: An Empirical Investigation, in: Public Finance, 1979, S. $36 \mathrm{ff}$.

Fiscal Policy and Crowding out in an Open Economy, unveröffentlichtes Diskussionspapier, 1977.

Some Notes on Fiscal Policy and Crowding Out, in: Australian Economic Review, 1979, S. 62 ff.

Fiscal Policy and Crowding Out, in: Posner, M. (Hrsg.), Demand Management, London, 1978, S. $167 \mathrm{ff}$.

National Income and the Price Level, New York u.a., 1962.

Macroeconomics. An Integration of Monetary, Search, and Income Theories, Chicago u.a., 1973. 
Barbosa, A.S.P.,

Barro, R.J.,

Barro, R.J.,

Blinder, A.S.,

Solow, R.M.,

Bookstaber, R.,

Brandmüller, S.,

Brenner, G. , Haury, C.E., Lipp, E.M., Brenner, G. , Lipp, E.M..

Brestel, H.,

Bruce, N. , Purvis, D.D.,

Brunner, K. ,

Brunner, K. ,

Brunner, K. , Meltzer, A.H.,

Buchanan, J.M.,
The Role of Wealth in the Money Demand Function: A Comment, in: Journal of Monetary Economics, 1979, S. 585 ff.

Are Government Bonds Net Wealth?, in: Journal of Political Economy, 1974, S. 1095 ff.

Public Debt and Taxes, noch nicht veröffentlicht, o.J.

Does Fiscal Policy Matter?, in: Journal of Public Economics, 1973, S. 319 ff.

The Effect of Inflation Uncertainty on 'Crowding Out', in: Journal of Macroeconomics, 1980 , S. 85 ff.

Zur Integration Zahlungsbilanztheoretischer Ansätze - Eine makroökonomische Analyse, Mainzer Dissertation, 1979.

Staatsverschuldung und Verfassung. Bestandsaufnahme und Neubesinnung, in: Finanzarchiv, 1980, S. $236 \mathrm{ff}$.

Beschäftigungspolitische Verantwortung der Finanzpolitik. Probleme bei ambivalenter Ursachendiagnose, Diskussionsbeiträge des Fachbereichs Wirtschaftswissenschaft der Universität des Saarlandes, Nr. A 7802, Juni 1978 .

Die Angst vor dem "Gelduntergang", in: Frankfurter Allgemeine Zeitung vom 6.6.1981.

Deficit Finance and 'First Round' Crowding-Out: A Correction and Further Clarification, in: Canadian Journal of Economics, 1979, S. $728 \mathrm{ff}$.

The "Monetarist Revolution" in Monetary Theory, in: Weltwirtschaftliches Archiv, 1970, S. 1 ff.

Falsche Leitbilder für die Politiker, in: Die Zeit, Nr. 501978 , s. 30.

Government, the Private Sector and Crowding Out, in: The Banker, 1976, S. 765 ff.

The Public Finances, Homewood, Ill., 1965. 
Buiter, w. ,

Buiter, W. , Tobin, J.,

Burrows, P:,

Butkiewicz, J.L.

Butkiewicz, J.L.,

Cansier, D.,

Carlson, M.K.,

Carlson, M.K., Spencer, R.W.,

Cassel, D.,

Cebula, R.J.,

Cebula, R.J., Carlos, C., Koch, J.V.,

Cebula, R.J.,
"Crowding out" and the Effectiveness of Fiscal Policy, in: Journal of Public Economics, 1977, S. 309 ff.

Debt Neutrality: A Brief Review of Doctrine and Evidence, zur Veroffentlichung vorgesehen in: Fürstenberg, G.M. von (Hrsg.), Social Security versus Private Saving in Post-industrial Socleties.

The Government Budget Constraint and the Monetarist-Keynesian-Debate, in: Cook, S.T., Jackson, P.M. (Hrsg.), Current Issues in Fiscal Policy, Oxford, 1979, s. $61 \mathrm{ff}$.

Some Macroeconomic Effects of Government Debt, Diss., University Microfilms International, Ann Arbor, Michigan, 1977.

Outside Wealth, the Demand for Money and the Crowding Out Effect, in:

Journal of Monetary Economics, 1979, S. 249 ff.

Einwände gegen die Wirksamkeit der Fiskalpolitik, in: Kredit und Kapital, 1977, S. 45 ff.

Large Federal Budget Deficits:

Perspective and Prosperity, in:

Federal Reserve Bank of St. Louis Review, Oktober 1976, S. 2 ff.

Crowding-Out and its Critics, in:

Federal Reserve Bank of St. Louis

Review, Dezember 1975, S. 2 ff.

Wachsende Staatsverschuldung

- Wohltat oder Plage?, in: List

Forum, 1980, S. 265 ff.

Cavaco-Silva, A.A., Economic Effects of Public Debt, London, 1977.

Deficit Spending, Expectations, and Fiscal Policy Effectiveness, in: Public Finance, 1973, S. $362 \mathrm{ff}$.

An Empirical Analysis of the "Crowding Out" Effect of Fiscal Policy in the United States and Canada, in: Kyklos, 1978, S. $424 \mathrm{ff}$.

The "Crowding Out" Effect of Federal Government Outlay Decisions: An Empirical Note, in: Public Choice, 1981 , S. 329 ff. 
Choudhry, N.N.,

Christ, C.F.,

Cohen, D., McMenamin, J.S.,

Currie, D.A.,

David, P.A., Scadding, J.L.,

Davidson, P.,

Dieckheuer, G.,

Dietzel, C.,

Dieckheuer, G. ,
Integration of Fiscal and Monetary Sectors in Econometric Models: A Survey of Theoretical Issues and Findings, in: International Monetary Fund, Staff Papers, 1976, S. $396 \mathrm{ff}$.

A Simple Macroeconomic Model with a Government Budget Restraint, in: Journal of Political Economy, 1968, S. $53 \mathrm{ff}$.

The Role of Fiscal Policy in a Financially Disaggregated Macroeconomic Model, in: Journal of Money, Credit, and Banking, 1978, s. $322 \mathrm{ff}$.

Monetary and Fiscal Policy and the Crowding-Out Issue, Diskussionspapier, Mai 1978.

Private Savings: Ultrarationality, Aggregation and "Denison's Law", in: Journal of Political Economy, 1974 , S. 225 ff.

The Crowding Out Effect. Monetarists vs Keynesians vs Keynes, Paper presented at the 53rd Annual Meetings of the Western Economic Association Conference in Hawaii, 1978.

Eine dynamische Analyse des "Crowdingout-Effektes" zusätzlicher Staatsausgaben, in: Finanzarchiv, 1974/75, S. $387 \mathrm{ff}$.

Möglichkeiten und Risiken einer kreditfinanzierten Stabilisierungspolitik, in: Wirtschaftsdienst, 1977, S. $553 \mathrm{ff}$.

Dieckheuer, G., Der Crowding-out-Effekt - zum gegenwärtigen Stand von Theorie und Empirie, in: Vierteljahreshefte zur Wirtschaftsforschung, 1980, S. $126 \mathrm{ff}$.

Das System der Staatsanleihen im zusammenhang der Volkswirtschaft betrachtet, Heidelberg, 1955, in: Diehl, K., Mombert, P. (Hrsg.), Ausgewählte Lesestücke zum Studium der politischen రkonomie, Neuedition von Hickel, R., Frankfurt, Berlin, Wien, 1980, S. $211 \mathrm{ff}$. 
Duwendag, D.,

Emrich, R.,

Fand, D. ,

Floyd, J.E., Heynes, J.A.,

Forte, F., Hochmann, H.M.,

Francke, H.H.,

Francke, H.H.,

Frey, R.L.,

Friedman, B.,

Friedman, M.,

Friedman, M. ,
Monetäre Grenzen der Staatsverschuldung, in: Duwendag, D., Siebert, H. (Hrsg.), Politik und Markt, Wirtschaftspolitische Probleme der $80 \mathrm{er}$ Jahre, Stuttgart, New York, 1980, S. $65 \mathrm{ff}$.

Die Theorie des Geldangebots in der geschlossenen und in der offenen Volkswirtschaft, Mainzer Dissertation, 1977.

Some Issues in Monetary Economics, in: Federal Reserve Bank of St. Louis Review, Januar 1970, S. $10 \mathrm{ff}$.

Deficit Finance and "First-Round" Crowding Out: A Clarification, in: Canadian Journal of Economics,1978, S. $97 \mathrm{ff}$.

Monetary and Fiscal Policy:

Ambiguities in Definitions, in: Haller, H., Recktenwald, H.C. (Hrsg.), Finanz- und Geldpolitik im Umbruch, Festschrift für R. Nöll von der Nahmer, Mainz, 1969, S. 357 ff.

Ober- und Untergrenzen der öffentlichen Verschuldung. Ein kritischer Kommentar zu Ausführungen von Wolfgang Stützel, in: Kredit und Kapital, 1979 , S. 362 ff.

Portfolioeffekte öffentlicher Kreditnahme, Berlin, 1981.

Infrastruktur. Grundlagen der Planung öffentlicher Investitionen, Tübingen, $1972^{2}$.

Crowding out or Crowding In?

Economic Consequences of Financing Government Deficits, in: Brookings Papers on Economic Activity, 1978, S. $593 \mathrm{ff}$.

Capitalism and Freedom, Chicago, 1962.

The Role of Monetary Policy, in: American Economic Review, 1968, S. $1 \mathrm{ff}$. 
Friedman, M.,

Friedman, M.,

Friedman, M. ,

Friedman, M., Heller, W.W., Friedman, M., Meiselman, D. ,

Friedrichs, H.,

Frowein, D. - K.,

Fürstenberg,G.M. van Public versus Private Spending: The Long-Term Consequences of Direct Crowding Out, in: derselbe (Hrsg.), The Government and Capital Formation, Cambridge, Mass., 1980, S. 243 ff.

Fürstenberg, G.M. von The Effect of Changing Size and Composition of Government Purchases on Potential Output, in: The Review of Economics and Statistics, 1980, S. $74 \mathrm{ff}$.

Fürstenberg, G.M. von The Government and Capital Formation: Malkiel, B.G., A Survey of Recent Issues, in: The Journal of Economic Literature, 1977, S. $835 \mathrm{ff}$.

Gandenberger, $0 ., \quad$ Zur Rationalität der öffentlichen Kreditnahme, in: Finanzarchiv, 1971, S. $369 \mathrm{ff}$.

Gandenberger, O., Öffentliche Verschuldung, II. Theoretische Grundlagen, in: Albers, W. u.a. (Hrsg.), Handwörterbuch der Wirtschaftswissenschaft, 5. Band, Stuttgart, Tübingen, Göttingen, 1980, S. 480 ff. 
Gordon, R.J.,

Gordon, R.J.,

Grossman, H.J. ,

Härtel, H.H.,

Hansen, B. ,

Hein, S.,

Holcombe, R.H., Jackson, J.D., Zardkoohi, A. , Hoyer, W. ,

Infante, E.F., Stein, J.L.,

Johnson, H.G.,

Kaminow, I.,

Karmann, A. Ketterer, K.-H.,
The Demand for and the Supply of Inflation, in: Journal of Law and Economics, 1975, S. 807 ff.

Output Fluctuations and Gradual

Price Adjustment, in: Journal of Economic Literature, 1981, S. $493 \mathrm{ff}$.

Was Keynes a "Keynesian"? A Review Article, in: Journal of Economic Literature, 1972 , S. 26 ff.

Die Kontroverse über die "richtige" Vollbeschäftigungsstrategie, in: Wirtschaftsdienst, 1980, S. $177 \mathrm{ff}$.

On the Effects of Fiscal and Monetary Policy: A Taxonomic Discussion, in: American Economic Review, 1973, S. 546 ff.

Deficits and Inflation, in: Federal Reserve Bank of St. Louis Review, Nr. 3, 1981, S. 3 ff.

A Tax Cut in a Multiple Security Model: Crowding-Out, Pulling-In and the Term Structure of Interest Rates, in: Journal of Finance, 1976 , S. $1185 \mathrm{ff}$.

The National Debt Controversy, in: Kyklos, 1981, s. $186 \mathrm{ff}$.

Vermögenseffekte des Geldes, Frankfurt am Main, Bern, Las Vegas, 1978.

Does Fiscal Policy Matter?, in: Journal of Monetary Economics, 1976 , S. 473 ff.

The Keynesian Revolution and the Monetarist Counter-Revolution, in: American Economic Review, Papers and Proceedings, Mai 1971, s. $1 \mathrm{ff}$.

The Myth of Fiscal Policy: The Monetarist View, in: Federal Reserve Bank of Philadelphia Business Review, Dezember 1969, S. $10 \mathrm{ff}$.

Staatsverschuldung und Investitionstätigkeit: Ist ein "Crowding out" die zwangsläufige Folge einer zunahme der Staatsverschuldung?, in: Göppl, H. , Henn, R. (Hrsg.), Geld, Banken und Versicherungen, Beiträge zum 1. Symposium Geld, Banken und Versicherungen an der Unıversität Karlsruhe vom 11.-13. Dez. 1980, Königstein/Ts., 1981, S.230 ff. 
Kath, D.,

Euba, N.,

Kennedy, P.E.,

Keran, M.W.,

Kern, H.,

Ketterer, K.-H., Karmann, A.,

Ketterer, K. - H., Vollmer, R.,

Keynes, J.M.,

Keynes, J.M.,

Kirner, W.,

Kitching, B.,

Kitterer, W. ,

Klausinger, H.,

Kloten, N., Barth, H.J., Ketterer, K. - H., Vollmer, R.,
Die makroökonomische Portfoliotheorie, in: Wirtschaftswissenschaftliches Studium, 1975, S. $458 \mathrm{ff}$.

Direct Wealth Effects in Macroeconomic Models: The Saving vs. the Definitional Approach, in: Journal of Money, Credit, and Banking, 1978, S. 94 ff.

Monetary and Fiscal Influence on Economic Activity - The Historical Evidence, in: Federal Reserve Bank of St. Louis Review, November 1969 , S. 5 ff.

Crowding-out durch Staatsverschuldung?, in: Wirtschaftsdienst, 1980 , S. 566 ff.

Einige theoretische und empirische Aspekte der Zinswirkungen der Staatsverschuldung, in: Karmann, A. (Mitverf.), ther Zins, Geldwert, Versicherungen und "kapitalistischer Geist", Königstein/Ts., 1981, S. 11 ff.

Bestimmungsgründe des Realzinsniveaus. Eine empirische Untersuchung für die Bundesrepublik Deutschland, in: Ehrlicher, $w$. (Hrsg.), Geldpolitik, Zins und Staatsverschuldung, Schriften des Vereins für Socialpolitik, Bd. 111, Berlin, 1981, S. 55 ff.

The General Theory of Employment, Interest and Money, London, 1936.

The General Theory of Employment, in: Quarterly Journal of Economics, $1936 / 37$, S. 209 ff.

Position des DIW, in: Vierteljahreshefte zur Wirtschaftsforschung, 1980, S. 29 ff.

Real "Crowding Out" as a Factor in the American Inflation-Recession, in: Zeitschrift für Nationalökonomie, 1972 , S. 289 ff.

Uber die Grenzen der Staatsverschuldung, Manuskript, 1980.

Rationale Erwartungen und die Theorie der Stabilisierungspolitik, Frankfurt am Main, Bern, Las Vegas, 1980 .

Zur Entwicklung des Geldwertes in Deutschland, Tübingen, 1980 . 
Koch, W.A.S.
Lang, E.

Korliras, P.G.,

Kühn, B. ,

Lachmann, W. ,

Landmann, O.,

Leijonhufvud, A. ,

Leipert, C. ,

Lerner, A.P.,

Lipp, E.M. ,

Littmann, K. ,

Machlup, F.,

Mackscheidt, K.

Crowding-out und staatliches debt management, in: WSI-Mitteilungen, 1981, S. $50 \mathrm{ff}$.

Bond-Financed Deficits and the Crowding-Out Hypothesis, Working Paper Nr. 37, University of Pittsburgh, 1976.

Rationale Erwartungen und Wirtschaftspolitik, Baden-Baden, 1979.

Crowding-out und die Frage nach der neutralen Geldpolitik, in: Kredit und Kapital, 1978, S. $451 \mathrm{ff}$.

Keynes in der heutigen Wirtschaftstheorie, in: Bombach, G., Ramser, H.-J., Timmermann, M., Wittmann, W. (Hrsg.), Der Keynesianismus I, Theorie und Praxis keynesianischer Wirtschaftspolitik, Berlin, Heidelberg, New York, 1976 , S. $133 \mathrm{ff}$.

On Keynesian Economics and the Economics of Keynes, New York, 1968.

Staatskonsum, staatliche Investitionen und die Produktivität staatlichen Handelns, in: Wirtschaftsdienst, 1979, S. $147 \mathrm{ff}$.

Functional Finance and the Federal Debt, in: Social Research, 1943, übersetzt in: Nowotny, E. (Hrsg.), ठffentliche Verschuldung, Stuttgart, New York, 1979, S. 87 ff.

Staatlicher Handlungsbedarf bei anhaltender Arbeitslosigkeit - Bemerkungen $\mathrm{zu}$ einer anhaltenden Kontroverse, in: Public Finance, 1980 , S. 155 ff.

Stand der Stabilisierungstheorie und Grenzen der Stabilisierungspolitik, in: Quartalshefte der österreichischen Girozentrale, $2-3 / 1978$, S. 35 ff.

The Effects of Fiscal Policy and the Choice of Definitions, in: Greentield, H.I. U.a. (Hrsg.), Theory for Economic Efficiency: Essays in Honor of Abba P. Lerner, Cambridge, Mass., London, 1979, S. 92 ff.

Crowding-Out als MaBstab für die Effizienz der fiscal policy?, in: Duwendag, D., Siebert, H. (Hrsg.), Politik und Markt, Wirtschaftspolitische Probleme der 80 er Jahre, Stuttgart, New York, 1980, S. $53 \mathrm{ff}$. 
Mayer, T.,

McCallum, B.T., Whitaker, J.K.,

Meade, J.E.,

Meister, J.,

Meister, J.,

Merklein, R.,

Metzger, R.,

Meyer, L.H.,

Meyer, L.H.,

Meyer, L.H.,

Meyer, L.H.,

Hart, W.R.,
Die Struktur des Monetarismus,

in: Ehrlicher, W., Becker, W. -D.

(Hrsg.), Die Monetarismus-Kontro-

verse, Beihefte zu Kredit und Kapital, Nr. 4, 1978, S. 9 ff.

Rational Expectations and Macroeconomic Stabilization Policy,

in: Journal of Money, Credit, and Banking, 1980, S. 716 ff.

The Effectiveness of Fiscal Feedback

Rules and Automatic Stabilizers

under Rational Expectations, in:

Journal of Monetary Economics, 1979, S. 171 ff.

The Balance of Payments, London, New York, 1952.

Konjunkturelle Effekte des Budgets - eine allokative Betrachtung, Referat, gehalten auf dem KongreB "Junge Wissenschaft und Wirtschaft" der Hanns Martin Schleyer-stiftung, Innsbruck, November 1980.

Budget und Konjunktur. Eine allokative ProzeBanalyse, Göttingen, 1981.

"Wenn die Lebensgeister trübe werden", in: Der Spiegel, 1978, Nr. $36 \mathrm{~s} .86 \mathrm{ff}$. und $\mathrm{Nr} .37 \mathrm{~s} .94 \mathrm{ff}$.

Zur Effizienz und Interdependenz der Geld- und Fiskalpolitik, Tübinger Dissertation, 1977.

Wealth Effects and the Effectiveness of Monetary and Fiscal Policies, in: Journal of Money, Credit, and Banking, 1974 , S. $481 \mathrm{ff}$.

A Balance Sheet Identity, the Government Financing Constraint, and the Crowding-Out Effect, in: Journal of Monetary Economics, 1975, S. $65 \mathrm{ff}$.

Financing Constraints and the Short-Run Response to Fiscal Policy, in: Federal Reserve Bank of St. Louis Review, Nr. 6 1980, S. $24 \mathrm{ff}$.

On the Effects of Fiscal and Monetary Policy: Completing the Taxonomy, in: American Economic Review, 1975, S. $762 \mathrm{ff}$. 
Minford, P.,

Peel, D.,

Modigliani, F., Ando, A.,

Monissen, H.G.,

Münnich, F.E.,

Musgrave, R.A.,

Muth, J.,

Neldner, M. ,

Neldner, M.,

Neumann, M.J.M.,

Neumann, M.J.M., Schröder, W. ,

Neumark, F.,

Nölling, W. ,

Ott, D.J.,

Ott, A.F.,
The Role of Monetary Stabilization Policy under Rational Expectations, in: The Manchester School, 1981, S. $39 \mathrm{ff}$.

Impacts of Fiscal Actions on Aggregate Income and the Monetarist Controversy: Theory and Evidence, in: Stein, J.L. (Hrsg.), Monetarism, Amsterdam, New York, Oxford, 1976, s. 17 ff.

Konsum und Vermögen. Analyse der Konsum-Vermögen-Relation im makroökonomischen Zusammenhang, Göttingen, 1968 .

Ist das Nachfragemanagement ibberholt?, Koreferat, in: Seidel, H., Butschek, F. (Hrsg.), Ist Arbeitslosigkeit unvermeidlich?, Wien, 1979, S. 99 ff.

Finanztheorie, Tủbingen, $1974^{2}$.

Rational Expectations and the Theory of Price Movements, in: Econometrica, 1961, S. 315 ff.

Einige problematische Aspekte der monetaristischen Stabilisierungspolitik, in: Konjunkturpolitik, 1975, S. $22 \mathrm{ff}$.

Aspekte monetaristischer Theorie: Eine Erwiderung, in: Konjunkturpolitik, 1975, S. $52 \mathrm{ff}$.

Rationale Erwartungen in Makromodellen, in: Zeitschrift für Wirtschafts - und Sozialwissenschaften, 1979, S. $371 \mathrm{ff}$.

Aspekte monetaristischer Theorie, in: Konjunkturpolitik, 1975, S. $36 \mathrm{ff}$.

Grundsätze und Arten der Haushaltsfuhrung und Finanzbedarfsdeckung, in: Gerloff, W., Neumark, F. (Hrsg.), Handbuch der Finanzwissenschaft, Erster Band, Tübingen, $1952^{2}$, S. $606 \mathrm{ff}$.

Staatsverschuldung ohne Ende?, in: Kredit und Kapital, 1979, S. $472 \mathrm{ff.}$.

Budget Balance and Equilibrium Income, in: Journal of Finance, 1965 , S. $71 \mathrm{ff}$. 
Ott, D.J.,
Ott, A.F.,

Patinkin, D.,

Paul, E.,

Peacock, A.T.,

Peacock, A.T.,

Shaw, G.K.,

Peffekoven, R. ,

Pesek, B.P.,

Saving, T.R.,

Peston, M. ,

Phelps, E.S.,

Phelps-Brown, H.,

Pohl, Reinhard,

Pohl, Reinhard,

Räth, N. ,
Fiscal Policy and the National

Debt, in: Smith, W.L., Teigen,

R.t. (Hrsg.), Readings in Money,

National Income, and Stabilization

Policy, Homewood, Ill., 19743,

S. $330 \mathrm{ff}$.

Money, Interest and Prices, New York, $1965^{2}$.

Der Crowding-Out-Effect, in:

Wirtschaftswissenschaftliches

Studium, 1977, S. 177 ff.

The Multiplier and the Valuation

of Government Expenditures, in:

Finanzarchiv, 1972, S. $418 \mathrm{ff}$.

Is Fiscal Policy Dead?, in:

Banca Nazionale del Lavoro

Quarterly Review, June 1978,

S. $107 \mathrm{ff}$.

రffentliche Finanzen, in: Vahlens

Kompendium der Wirtschaftstheorie und Wirtschaftspolitik, München, 1980 , S. 419 ff.

Money, Wealth, and Economic Theory, New York, London, 1967.

An Aspect of the Crowding out

Problem, in: Oxford Economic Papers, 1981 , S. $19 \mathrm{ff}$.

Phillips Curves, Expectation of Inflation and Optimal Unemployment Over Time, in: Economica, 1967, S. $254 \mathrm{ff}$.

A Non-Monetarist View of the Pay Explosion, in: The Three Banks Review, 1975, s. $3 \mathrm{ff}$.

Die Auswirkungen der Staatsverschuldung auf die Finanzmärkte, in: Beihefte der Konjunkturpolitik, Heft 27,1980, S. $85 \mathrm{ff}$.

Einige geldtheoretische und geldpolitische Probleme des crowding-out des privaten Sektors durch den Staat, in: Ehrlicher, W. (Hrsg.), Geldpolitik, Zins und Staatsverschuldung, Schriften des Vereins für Socialpolitik, Band 111, 1981, s. 263 ff.

Die Zwangsanleihe als finanzpolitisches Instrument, St. Gallen, 1980. 
Ramser, H.J.,

Richebächer, K. ,

Rose, M. ,

Sachverständigenrat

zur Begutachtung

der gesamtwirt-

schaftlichen Ent-

wicklung,

Sachverständigenrat

zur Begutachtung

der gesamtwirt-

schaftlichen Ent-

wicklung,

Sachverständigenrat zur Begutachtung

der gesamtwirtschaftlichen Entwicklung,

Sachverständigenrat zur Begutachtung

der gesamtwirt-

schaftlichen Ent-

wicklung,

Sachverständigenrat

zur Begutachtung

der gesamtwirt-

schaftlichen Ent-

wicklung,

Sachverständigenrat zur Begutachtung

der gesamtwirtschaftlichen Entwicklung,

Say, J.B.,

Scarth, W.M. ,

Schäfer, C.,
Rationale Erwartungen und Wirtschaftspolitik, in: Zeitschrift für die gesamte staatswissenschaft, 1978 , S. 57 ff.

Das süBe Gift des Deficit Spending, Währungen und Kreditmärkte, $\mathrm{Nr}, 64$, Frankfurt, 1975.

Finanzwissenschaftliche Makrotheorie, München, 1980 .

Jahresgutachten 1975, Bundestagsdrucksache $7 / 4326$.

Jahresgutachten 1976/77, Bundestagsdrucksache 7/5902.

Mehr Wachstum, mehr Beschäftigung, Jahresgutachten 1977/78, Stuttgart, Mainz, 1977.

Jahresgutachten 1978/79, Bundestagsdrucksache $8 / 2313$.

Jahresgutachten 1980/81, Bundestagsdrucksache $9 / 17$.

Vor Kurskorrekturen, Sondergutachten vom 4. Juli 1981.

Traité d'économie politique, Tome I, Paris, $1826^{5}$.

A Note on the "Crowding Out" of Private Expenditures by Bond-Financed Increases in Government Spending, Journal of Public Economics, 1976, S. $385 \mathrm{ff}$.

Zur Notwendigkeit und Legitimation einer mittelfristigen beschäftigungssichernden Finanzpolitik, in: WSI-Mitteilungen, 1980 , S. $634 \mathrm{ff}$. 
Schips, B., Jaeger, F..

Schmidt, K.,

Schmidt, K.,

Schmidt, K.,

Schmölders, G. ,

Shiller, R.,

Siebke, J.,

Siebke, J.,

Siebke, J.,

Knoll, D.,

Schmidberger, W.D.,

Sievert, O.,
Okonomische Analyse der crowdingout-Wirkungen der Staatsverschuldung in der Schweiz, Vortrag, gehalten auf der Tagung "Geld, Banken und Versicherungen" in Karlsruhe vom $11 .-13 \cdot 12.1980$.

రffentliche Neuverschuldung in der Rezession - neue Erfahrungen und alte Einsichten, in: రffentliche Verschuldung und Kapitalmarkt, Schriftenreihe des Instituts für Kapitalmarktforschung an der J.W. Goethe-Universität, Frankfurt, 1976 , s. 45 ff.

Finanzpolitik für mehr wirtschaftliches Wachstum, in: Bohley, P.. Tolkemitt, G. (Hrsg.), Wirtschaftswissenschaft als Grundlage staatlichen Handelns, Tübingen,1979, S. 115 ff.

Diskussionsbeitrag, in: Beihefte der Konjunkturpolitik, Heft 27 , 1980 , S. 120.

Finanzpsychologie, in: Finanzarchiv, 1951, S. 1 ff.

Rational Expectations and the Dynamic Structure of Macroeconomic Models, in: Journal of Monetary Economics, 1978, S. $1 \mathrm{ff}$.

Die Berücksichtigung der Budgetbeschränkung des Staates in dem Keynesianischen Makrosystem, in: das wirtschaftsstudium, 1975, S. $490 \mathrm{ff}$. und $538 \mathrm{ff}$.

Der "crowding-out"-effect in einem Portfolio-Makromodell, in: Albach, H. u.a. (Hrsg.), Quantitative Wirtschaftsforschung. Wilhelm Krelle zum 60. Geburtstag, Tübingen, 1977, S. $655 \mathrm{ff}$.

Theoretische Grundlagen des crowding out Effektes, in: Ehrlicher, W. (Hrsg.), Geldpolitik, Zins und Staatsverschuldung, Schriften des Vereins für Socialpolitik, Band 111, 1981, S. 227 ff.

LäBt sich konjunkturgerechtes Verhalten der öffentlichen Gebietskörperschaften messen?, in: Quartalshefte der österreichischen Girozentrale, $2-3 / 1978$, S. $53 \mathrm{ff}$. 
Sievert, $0 .$,

Sievert, $0 .$,

Sievert, 0.,

Silber, W.L.,

Smith, A.,

Smith, D.,

Sowell, T.,

Spahn, H.P.,

Spencer, R.W., Yohe, W.P.,

Stevens, N.A.,

Stützel, W. ,
Arbeitslosigkeit und Lohnhöhe, in: Seidel, H., Butschek, F. (Hrsg.), Ist Arbeitslosigkeit unvermeidlich?, Wien, 1979, S. $13 \mathrm{ff}$.

Die Steuerbarkeit der Konjunktur durch den Staat, in: Weizsäcker, C.C. viun (Hrsg.), staat und Wirtschaft, Schriften des Verreins für Socialpolitik, Bd. 102, Berlin, 1979, S. $809 \mathrm{ff}$.

Position des Sachverständigenrates, in: Vierteljahreshefte zur Wirtschaftsforschung, 1980 , S. $18 \mathrm{ff}$.

Fiscal Policy in IS-LM-Analysis: A Correction, in: Journal of Money, Credit, and Banking, 1970, s. $461 \mathrm{ff}$.

An Inquiry into the Nature and Causes of the Wealth of Nations, London, 1776, Campbell, R.H., Skinner, A.S. (Hrsg.), Vol. 1, Oxford, 1976.

Is Deficit Spending Practical?, in: Harvard Business Review, Vol. 18, 1939 .

Say's Law. An Historical Analysis, Princeton, N.J., 1972.

Keynes in der heutigen wirtschaftspolitik, in: Bombach, G., Ramser, H.-J., Timmermann, M., Wittmann, W. (Hrsg.), Der Keynesianismus I, Theorie und Praxis keynesianischer Wirtschaftspolitik, Berlin, Heidelberg, New York, 1976, S. 211 ff.

The "Crowding Out" of Private Expenditures by Fiscal Policy Actions, in: Federal Reserve Bank of St. Louls Review, Oktober 1970, wiederabgedruckt in: Boorman, J.T., Havrilesky, T.M., Money Supply, Money Demand and Macroeconomic Models, Northbrook, 1972, S. 429 ff.

Government Debt Financing - Its Effects in View of Tax Discounting, in: Federal Reserve Bank of St. Louis Review, Nr. 7 1979, S. $11 \mathrm{ff}$.

Ober- und Untergrenzen der öffentlichen Verschuldung, in: Kredit und Kapital, 1978, S. 429 ff. 
Sundararajan, V., Thakur, S.,

Surminski, A.,

Svindland, E.,

Taylor, C.T.,

Teschner, M.,

Tobin, J.,

Tobin, J.,

Tobin, J.,

Tobin, J.,
Public Investment, Crowding Out, and Growth: A Dynamic Model Applied to India and Korea, in: Staff Papers, International Monetary Fund, 1980, S. 814 ff.

Was die Deutschen fürchten, in: Frankfurter Allgemeine Zeitung vom 31.5.1978.

Staatsausgaben und ihre Finanzierung. Einige elementare Bemerkungen $z u$ den Grundlagen der Crowding-out-Analyse, in: Vierteljahreshefte zur Wirtschaftsforschung, 1980, S. $148 \mathrm{ff}$.

Crowding Out: Its Meaning and Significance, in: Cook, S.T., Jackson, P.M. (Hrsg.), Current Issues in Fiscal Policy, Oxford, 1979 , S. 86 ff.

Hält die Crowding-out-These kreislauftheoretischer Kritik stand?, in: Vierteljahreshefte zur Wirtschaftsforschung, 1980, S. $121 \mathrm{ff}$.

An Essay on the Principles of Debt Management, in: Fiscal and Debt Management Policies, 1963, übersetzt: Grundsätze der Geld- und Staatsschuldenpolitik, Baden-Baden, 1978 .

The Equilibrium Approach to Monetary Theory, in: Journal of Money, Credit, and Banking, 1969, S. $15 \mathrm{ff}$. , ubersetzt: Ein allgemeiner Gleichgewichtsansatz zur Geldtheorie, in: Brunner, K., Monissen, H.G., Neumann, M.J.M. (Hrsg.), Geldtheorie, Köln, 1974, S. $219 \mathrm{ff}$.

Friedman's Theoretical Framework, in: Gordon, R.J. (Hrsg.), Milton Friedman's Monetary Framework. A Debate with His Critics, Chicago, London, 1974, S. 77 ff.

Deficit Spending and Crowding out in Shorter and Longer Runs, in: Greenfield, H.I. U.a. (Hrsg.), Theory for Economic Efficiency: Essays in Honor of Abba P. Lerner, Cambridge, Mass., London, 1979, S. $217 \mathrm{ff}$. 
Tobin, J., Buiter, W.,

Tobin, J., Buiter, W.,

Vane, H.R., Thompson, J.L., Verde, A.,

Walther, H.,

Westphal, U.,

Willms, M.,

Wilson, T.

Wissenschaftlicher Beirat beim Bundesministerium der Finanzen,

woglom, G. ,

Yawitz, J.,

Zahn, F.,
Long-run Effects of Fiscal and

Monetary Policy on Aggregate

Demand, in: Stein, J.L. (Hrsg.), Monetarism, Amsterdam, New York, Oxford, 1976, s. $273 \mathrm{ff}$.

Fiscal and Monetary Policies, Capital Formation, and Economic Activity, in: Fürstenberg, G.M. von (Hrsg.), The Government and Capital Formation, Cambridge, Mass., 1980 , S. $73 \mathrm{ff}$.

Monetarism. Theory, Evidence and Policy, Oxford, 1979.

Crowding out Once More: An Attempt to Estimate Its Size in Italy,

1974-1977, in: Review of the Economic Conditions in Italy, 1979 , S. $309 \mathrm{ff}$.

öffentliche Verschuldung und

"Crowding-out" Effekte, in:

Nowotny, E. (Hrsg.), Öfentliche

Verschuldung, stuttgart, New York, 1979 , S. 65 ff.

Empirische Aspekte des Crowding-out, in: Ehrlicher, W. (Hrsg.), Geldpolitik, Zins und Staatsverschuldung, Schriften des Vereins für Socialpolitik, Band 111, Berlin, 1981, s. $209 \mathrm{ff}$.

Volkswirtschaftliche Wirkungen einer zunehmenden Staatsverschuldung, in: Wirtschaftsdienst, 1978, S. $439 \mathrm{ff}$.

Crowding Out: The Real Issues, in: Banca Nazionale del Lavoro Quarterly Review, September 1979, S. $227 \mathrm{ff}$.

Zum Begriff der öffentlichen Investitionen, Bonn, 1980 .

First-Round "Crowding Out" in a Generalized IS-LM-Model, in:

Journal of Macroeconomics, 1979, S. $119 \mathrm{ff}$.

Economically Relevant Wealth: Its Specification and Role in Consumption, Diss., University Microfilms, Ann Arbor, Michigan, 1972.

A Flow of Funds Analysis of Crowding Out, in: Southern Economic Journal, 1978 , S. 136 ff. 
Ziffzer, S.

Ziffzer, S.

Zundel, M. ,

Zwick, B. , 8konomische, finanzwirtschaftliche, politisch-psychologische und gesetzliche Grenzen der Kreditaufnahme des Bundes, der Länder und der Gemeinden, Forschungsinstitut fur Wirtschaftspolitik an der Universität Mainz, unveroffentlicht, 1979.

Grenzen der Staatsverschuldung, 1n: Zeitschrift fur das gesamte Kreditwesen, 1980 , S. $500 \mathrm{ff}$.

EinfluB der öffentlichen Güter auf den privaten Konsum, Bochumer Dissertation, 1978.

"Snapback" and "Crowding out" Effects in Monetary and Fiscal Policy: Explanation and Interrelation, in: Journal of Money, Credit, and Banking, 1974, S. 559 ff. 
Christian Schiller - 978-3-631-75197-8

Downloaded from PubFactory at 01/11/2019 07:15:17AM

via free access 
Christian Schiller - 978-3-631-75197-8

Downloaded from PubFactory at 01/11/2019 07:15:17AM

via free access 


\section{FINANZWISSENSCHAFTLICHE SCHRIFTEN}

Band 1 Werner Steden: Finanzpolitik und Einkommensverteilung. Ein Wachstums- und Konjunkturmodell der Bundesrepublik Deutschland. 1979.

Band 2 Rainer Hagemann: Kommunale Finanzplanung im föderativen Staat. 1976.

Band 3 Klaus Scherer: Maßstäbe zur Beurteilung von konjunkturellen Wirkungen des öffentlichen Haushalts. 1977.

Band 4 Brita Steinbach: „Formula Flexibility" - Kritische Analyse und Vergleich mit diskretionärer Konjunkturpolitik. 1977.

Band 5 Hans-Georg Petersen: Personelle Einkommensbesteuerung und Inflation. Eine theoretisch-empirische Analyse der Lohn- und veranlagten Einkommensteuer in der Bundesrepublik Deutschland. 1977.

Band 6 Friedemann Tetsch: Raumwirkungen des Finanzsystems der Bundesrepublik Deutschland. Eine Untersuchung der Auswirkungen der Finanzreform von 1969 auf die Einnahmenposition der untergeordneten Gebietskörperschaften und ihrer regionalpolitischen Zieladäquanz. 1978.

Band 7 Wilhelm Pfähler: Normative Theorie der fiskalischen Besteuerung. Ein methodologischer und theoretischer Beitrag zur Integration der normativen Besteuerungstheorie in der Wohlfahrtstheorie. 1978.

Band 8 Wolfgang Wiegard: Optimale Schattenpreise und Produktionsprogramme für öffentliche Unternehmen. Second-Best-Modelle im finanzwirtschaftlichen Staatsbereich. 1978.

Band 9 Hans P. Fischer: Die Finanzierung des Umweltschutzes im Rahmen einer rationalen Umweltpolitik. 1978.

Band 10 Rainer Paulenz: Der Einsatz finanzpolitischer Instrumente in der Forschungs- und Entwicklungspolitik. 1978.

Band 11 Hans-Joachim Hauser: Verteilungswirkungen der Staatsverschuldung. Eine kreislauftheoretische Inzidenzbetrachtung. 1979.

Band 12 Gunnar Schwarting: Kommunale Investitionen. Theoretische und empirische Untersuchungen der Bestimmungsgründe kommunaler Investitionstätigkeit in Nordrhein-Westfalen 1965-1972. 1979.

Band 13 Hans-Joachim Conrad: Stadt-Umland-Wanderung und Finanzwirtschaft der Kernstädte. Amerikanische Erfahrungen, grundsätzliche Zusammenhänge und eine Fallstudie für das Ballungsgebiet Frankfurt am Main. 1980.

Band 14 Cay Folkers: Vermögensverteilung und staatliche Aktivität. Zur Theorie distributiver Prozesse im Interventionsstaat. 1981.

Band 15 Helmut Fischer: US-amerikanische Exportförderung durch die DISC-Gesetzgebung. 1981.

Band 16 Gijnter Ott: Einkommensumverteilungen in der gesetzlichen Krankenversicherung. Eine quantitative Analyse. 1981.

Band 17 Johann Hermann von Oehsen: Optimale Besteuerung. (Optimal Taxation). 1982.

Band 18 Richard Kössler: Sozialversicherungsprinzip und Staatszuschüsse in der gesetzlichen Rentenversicherung. 1982.

Band 19 Hinrich Steffen: Zum Handlungs- und Entscheidungsspielraum der kommunalen Investitionspolitik in der Bundesrepublik Deutschland. 1983.

Band 20 Manfred Scheuer: Wirkungen einer Auslandsverschuldung des Staates bei flexiblen Wechselkursen. 1983.

Band 21 Christian Schiller: Staatsausgaben und crowding-out-Effekte. Zur Effizienz einer Finanzpolitik keynesianischer Provenienz. 1983. 
Müthing, Michacl

\section{WACHSTUMSORIENTIERTE UNTERNEHMENSPLANUNG}

Ein computergestütztes Planungsmodell unter spezieller Berücksichtigung von

Wachstumsschranken

Frankfurt/M., Bern, Las Vegas, 1981. IV, $254 \mathrm{~S}$.

Europäische Hochschulschriften: Reihe 5, Volks- und Betricbswirtschaft. Bd. 316

ISBN 3-8204-6925-7

br. sFr. 59.-

Effiziente Unternehmensplanung erfordert methodisches Vorgehen, das durch die Verwendung computergestützter Planungsmodelle erleichtert wird. Vor allem für strategische Planungszwecke wie das Unternehmenswachstum sind an derartige Modelle besondere Anforderungen zu stellen, um sie realitätsnah und problemrelevant zu gestalten und ihre Annahme durch die Planungsträger sicherzustellen. Schwerpunkt dieser Arbeit ist die Entwicklung einer Modellstruktur, die diesen Kriterien entspricht, um unter Berücksichtigung von Wachstumsschranken die Auswahl geeigneter Wachstumsstrategien zu unterstützen und Schwachstellen der geplanten Entwicklung aufzuzeigen.

Aus dem Inhalt: Die betriebliche Wachstumstheorie - Informationsgewinnung und Modellgestaltung im Rahmen wachstumsorientierter Unternehmensplanung - Ein dynamisches Planungsmodell - Demonstration des Modells anhand von Fallstudien.

Köllner, Lutz

\section{VON DER PREUSSISCHEN STAATSBANK ZUM EUROPÄISCHEN WÄHRUNGSSYSTEM}

Hundert Jahre Währung und Politik in Deutschland und Europa

Frankfurt/M., Bern, Las Vegas, 1981. II, $110 \mathrm{~S}$.

Europäische Hochschulschriften: Reihe 5, Volks- und Betriebswirtschaft. Bd. 333

ISBN 3-8204-6957-5

br. sFr. 27.-

In hundert Jahren gab es in Deutschland 3 (mit der DDR 4) Neugründungen der Zentralbank und damit zusammenhängende Währungsreformen. 1871/74 wurde die Reichsgoldwährung geschaffen, 1923/24 und 1948 erfolgten die Liquidation der beiden Kriege. 1957 entstand die Deutsche Bundesbank. Das währungspolitische Instrumentarium und die währungspolitischen Ziele - im Stabilitätsgesetz von 1967 festgelegt - wandelten sich mehrfach. Vom währungspolitischen Versuch nach Weltgeltung führte der Weg zur mühevollen Kooperation in Westeuropa, deren derzeitiger Ausdruck die Bremer und Bonner Beschlüsse zur europäischen Währungspolitik von 1979 sind, die 1981 zu einem Europäischen Währungsfonds führen sollen.

Aus dem Inhalt: Die Reichswährungsreform von 1871/74 - Das Ende der Goldwährung 1914 - Kriegsfinanzen - Grosse Inflation und Währungsreform 1923/24 - Arbeitsbeschaffung und Aufrüstung - Die Reichsbank im 2. Weltkrieg - Währungsreform 1948 - Europäische Währungspolitik.

\section{Verlag Peter Lang $\cdot$ Bern und Frankfurt am Main}

Auslieferung: Verlag Peter Lang AG. Jupiterstr. 15. CH-3000 Bern 15

Telefon $(0041 / 31) 32$ II 22. Telex verl ch 32420

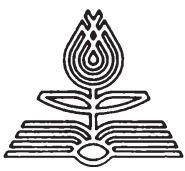

